André Cury Maiali

\title{
Controle Ótimo Estocástico a Tempo Discreto e Espaço de Estado Contínuo Aplicado \\ a Derivativos
}

Área de Concentração: Engenharia de Sistemas

Tese apresentada ao Departamento de Engenharia de Telecomunicações e Controle da Escola Politécnica da Universidade de São Paulo para a obtenção do título de Doutor em Engenharia.

Orientador: Prof. Dr. Oswaldo Luiz do Valle Costa

São Paulo, 2006 
Este exemplar foi revisado e alterado em relação à versão original, sob responsabilidade única do autor e com a anuência de seu orientador.

São Paulo, 18 de julho de 2006.

Assinatura do autor

Assinatura do orientador

FICHA CATALOGRÁFICA

Maiali, André Cury

Controle ótimo estocástico a tempo discreto e espaço de estado contínuo aplicado a derivativos I A.C. Maiali. -- ed.rev. -São Paulo, 2006.

$135 \mathrm{p}$.

Tese (Doutorado) - Escola Politécnica da Universidade de São Paulo. Departamento de Engenharia de Telecomunicações e Controle.

1.Controle ótimo 2. Derivativos 3. Processos estocásticos I. Universidade de São Paulo. Escola Politécnica. Departamento de Engenharia de Telecomunicações e Controle Il.t 


\section{Agradecimentos}

(em ordem alfabética)

Aos meus pais, Antônio e Penha, pela minha educação.

Aos meus grandes amigos, Daniela Akizuki e Germano Capistrano Bezerra, cuja ajuda tornou possível a realização desta tese.

Ao Prof. Dr. Oswaldo Luiz do Valle Costa, pelos ensinamentos, pela paciência e pela confiança. 
À minha esposa, Fabiana, e aos nossos filhos, Giulia e Enrico. 


\section{Resumo}

Nesta tese abordamos o problema do hedging de mínima variância de derivativos em mercados incompletos usando a teoria de controle ótimo estocástico com critério quadrático de otimização.

Desenvolvemos um modelo geral de apreçamento e hedging de derivativos em mercados incompletos, a tempo discreto, que é capaz de acomodar qualquer tipo de payoff com característica européia que dependa de $n$ ativos de risco. Nesse modelo, o mercado pode apresentar diferentes modos de operação, o que foi formalizado matematicamente por meio de uma cadeia de Markov.

Também desenvolvemos um modelo geral de apreçamento e hedging de derivativos em mercados incompletos, a tempo discreto e espaço de estados contínuo, que é capaz de acomodar qualquer tipo de payoff com característica européia que dependa de um ativo de risco cujos retornos sejam representados por um processo de difusão com saltos.

Desenvolvemos, ainda, expressões analíticas fechadas para o apreçamento e hedging de uma opção de compra européia vanilla em duas situações: (1) quando os retornos do ativo de risco são representados por um processo de difusão com saltos, e (2) quando os retornos do ativo de risco são representados por um processo de Wiener.

Por fim, realizamos simulações numéricas para o controle (hedging) de uma opção de compra européia vanilla quando os retornos do ativo de risco são representados por um processo de Wiener, e comparamos os resultados obtidos com a estratégia de controle derivada do modelo de Black \& Scholes. 


\section{Abstract}

In this thesis we approach the mean-variance hedging problem of derivatives in incomplete markets employing the theory of stochastic optimal control with quadratic optimization criteria.

We developed a general derivatives pricing and hedging model in incomplete markets, in discrete time, capable of accommodating any type of European payoff contingent on $n$ risky assets. In this model, the market may exhibit different operating modes, which were mathematically formalized by means of a Markov chain.

We also developed a general derivatives pricing and hedging model in incomplete markets, in discrete time and continuous state space, capable of accommodating any type of European payoff contingent on one risky asset whose returns are described by a jump diffusion process.

Even further, we developed closed-form analytical expressions for the pricing and hedging of a European vanilla call option in two situations: (1) when the risky asset returns are described by a jump diffusion process, and (2) when the risky asset returns are described by a Wiener process.

Finally, we simulated the control (hedging) of a European vanilla call option when the risky asset returns are described by a Wiener process, and compared the results to those obtained with the control strategy derived from the Black \& Scholes model. 


\section{Sumário}

1 Introdução 9

1.1 Motivação Para a Tese . . . . . . . . . . . . . . . . . . . . . 9

1.2 Objetivo da Tese . . . . . . . . . . . . . . . . . . 10

1.3 Estrutura da Tese . . . . . . . . . . . . . . . . . . . 10

1.4 Contribuições Originais . . . . . . . . . . . . . . . . . 11

2 Revisão Bibliográfica 13

2.1 Conceitos Básicos . . . . . . . . . . . . . . . . . 13

2.2 Estratégias de Controle (Hedging) com Critérios Quadráticos em

Tempo Contínuo . . . . . . . . . . . . . . . . 15

2.3 Estratégias de Controle (Hedging) com Critérios Quadráticos em Tempo Discreto . . . . . . . . . . . . . . . . . . . . . 18

3 Modelos em Tempo Discreto 19

3.1 Modelo Geral . . . . . . . . . . . . . . . . . . . . . . . . . . . . . 19

3.1 .1 Definições . . . . . . . . . . . . . . . . . . . . . 20

3.1.2 Descrição dos Elementos do Modelo Geral . . . . . . . . . 22

3.1.3 Formulação do Problema de Hedging de Mínima Variância 23

3.1.4 Variance Optimal Measure. . . . . . . . . . . . . . . 26

3.1 .5 Cálculo de $H(\tau) \ldots \ldots \ldots \ldots \ldots$

3.1 .6 Uma Interpretação Para $H(\tau) \ldots \ldots \ldots \ldots$

3.2 Modelo com $n$ Ativos de Risco sem Cadeia de Markov . . . . . . 29

3.3 Modelo com Um Ativo de Risco sem Cadeia de Markov . . . . . 31

4 Modelo em Tempo Discreto, com Espaço de Estados Contínuo, com Um Ativo de Risco e sem Cadeia de Markov 33

4.1 Conceitos Preliminares . . . . . . . . . . . . . . . . . . 33

4.2 Equações de Estado Para $\Delta X$ num Espaço de Estados Contínuo 36

4.3 Resultados Principais . . . . . . . . . . . . . . . . . 40

5 Aplicação 42

5.1 Opção de Compra Européia Vanilla com Jumps . . . . . . . . . . 42

5.2 Opção de Compra Européia Vanilla sem Jumps . . . . . . . . . . 46

6 Simulações Numéricas $\quad 49$

6.1 Estrutura das Simulações . . . . . . . . . . . . . . . . . 49

6.2 Resultados das Simulações . . . . . . . . . . . . . . . . . . 52

6.3 Discussão dos Resultados . . . . . . . . . . . . . . . . 65 
7 Conclusões e Pesquisas Futuras

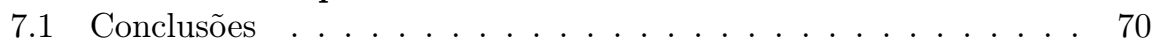

7.2 Pesquisas Futuras . . . . . . . . . . . . . . . . 72

A Demonstrações 


\section{Capítulo 1}

\section{Introdução}

Neste capítulo, inicialmente apresentamos o problema que serviu de motivação para esta tese e, em seguida, descrevemos qual é o objetivo a ser atingido. Na sequência, descrevemos a forma como esta tese está estruturada, discorrendo brevemente sobre o conteúdo de cada capítulo. Por fim, apontamos de forma objetiva quais são as contribuições originais contidas neste trabalho.

\subsection{Motivação Para a Tese}

Um derivativo financeiro $H$ é um instrumento financeiro cujo valor depende do valor de outros instrumentos financeiros mais elementares, chamados de ativos base ou ativos objeto. Em termos matemáticos, sendo $S(t)$ um vetor contendo os preços dos ativos base, o valor do derivativo financeiro no instante de tempo $t$ é representado por uma variável aleatória $H(t, S(t)), \mathcal{F}_{t}$-mensurável.

Problemas fundamentais da área de finanças matemáticas são o de apreçamento e o de hedging de derivativos financeiros empregando-se estratégias dinâmicas envolvendo os ativos base existentes no mercado.

Os problemas de apreçamento e de hedging podem ser formulados da seguinte forma: qual o preço pelo qual o vendedor do derivativo $H$ deve vendê-lo ao comprador? Tendo vendido o derivativo, que portfólio contendo os ativos base do mercado o vendedor deve criar para replicar esse derivativo, protegendose, portanto, de perdas no instante de tempo $T$ geradas pelo comportamento estocástico de $S(T)$ ?

Assim, seguindo essa lógica, consideremos uma instituição financeira que, no instante de tempo $t_{0}$, venda a seus clientes um contrato financeiro derivativo cujo valor em $T$, instante de seu vencimento, seja $H(T, S(T)$ ) (chamado de payoff do derivativo). Para que esta instituição possa reduzir sua exposição a risco financeiro, protegendo-se das perdas estocásticas mencionadas acima, ela precisa ser capaz de replicar o derivativo financeiro vendido, ou seja, ela precisa ser capaz de construir uma estratégia auto-financiável que use os instrumentos financeiros existentes no mercado, e que gere um portfólio cujo valor no instante de tempo $T$ seja o mais próximo possível de $H(T, S(T))$.

Esse problema tem altíssima relevância prática, pois está relacionado à possibilidade de ocasionar perdas financeiras vultosas aos vendedores de instrumentos derivativos caso as ações de controle apropriadas não sejam tomadas correta- 
mente. Assim, por sua importância, esse problema provê a motivação para esta tese. No item a seguir, explicamos com mais detalhes como esse problema será abordado.

\subsection{Objetivo da Tese}

O objetivo desta tese é explorar e resolver o problema descrito acima. A abordagem adotada é a de se determinar o hedging de mínima variância de um contrato derivativo não-replicável usando métodos de programação dinâmica estocástica em tempo discreto, com espaço de estados contínuo. Entende-se por hedging de mínima variância aquele que busca minimizar o valor esperado do desvio quadrático entre o valor do portfólio gerado pela estratégia de hedging e o valor de mercado $H(T, S(T))$ do contrato derivativo não-replicável (i.e., contrato derivativo para o qual não há estratégia replicante).

Conforme a descrição a ser apresentada no Capítulo 2, muito já se explorou com relação ao problema de hedging de mínima variância em tempo e estado contínuo, incluindo-se aí modelos em que os retornos dos ativos base apresentam volatilidade estocástica e/ou descontinuidades, também chamadas de saltos. Poucos trabalhos abordaram esse problema em tempo e espaço de estados discretos. Não foram encontrados na literatura trabalhos explorando o caso em que o domínio do tempo é discreto e o espaço de estados é contínuo. Entretanto, essa é a situação que mais se aproxima de um modelo apropriado para descrever o problema real de hedging de um contrato derivativo.

De fato, os processos de tomada de decisão de investimento (rebalanceamento de portfólio) ocorrem em instantes de tempo determinados que assumem valores num domínio discreto. Portanto, modelar o tempo como uma variável pertencente a um domínio discreto é uma decisão correta. Os preços dos ativos, por sua vez, também assumem valores num domínio discreto, pois são quantizados, i.e., eles podem assumir apenas determinados valores compatíveis com os chamados ticks de mercado, que são definidos como o módulo da mínima variação de preço entre duas cotações diferentes (o preço de uma ação jamais será $\sqrt{150}$, por exemplo). Porém, embora os preços dos ativos sejam quantizados, os ticks de mercado são em geral pequenos o suficiente para permitir que esses preços assumam muitos valores diferentes, fazendo com que um espaço de estados contínuo seja o melhor modelo para descrevê-los. Portanto, modelar os preços por processos estocásticos que assumam valores sobre um continuum é uma decisão correta.

Assim, nesta tese exploramos o problema de hedging de mínima variância por meio de modelos que descrevam mais adequadamente as condições e práticas reais envolvidas no mercado financeiro. São empregados modelos com dinâmicas contendo parâmetros estocásticos e descontinuidades (saltos).

\subsection{Estrutura da Tese}

Esta tese está estruturada da seguinte forma. No Capítulo 1, apresentamos a motivação para o desenvolvimento da tese, definimos o objetivo a ser atingido com os estudos realizados, descrevemos sumariamente a forma como esta tese está estruturada e, por fim, destacamos objetivamente os resultados originais 
obtidos na tese.

No Capítulo 2, apresentamos uma breve revisão teórica da literatura sobre o controle ótimo de um portfólio que busque replicar o valor de um contrato derivativo, i.e., de um portfólio que busque ser o "melhor" hedging para um derivativo.

No Capítulo 3, apresentamos resultados gerais para o apreçamento e hedging de um derivativo qualquer com característica européia (i.e., que apenas pode ser exercido no seu vencimento) cujo payoff dependa de $n$ ativos de risco, num modelo de mercado que opere em tempo discreto, em diferentes modos, que são representados por uma cadeia de Markov, e que contenha $n$ ativos de risco e um outro livre de risco. Essa abordagem também se aplica ao caso de derivativos com payoff dependente da trajetória. Apenas os derivativos com característica americana (i.e., que podem ser exercidos a qualquer momento até seu vencimento) estão excluídos dessa abordagem.

Ainda no Capítulo 3, efetuamos simplificações no modelo geral. Mais especificamente, inicialmente adotamos um modelo de mercado que opere em apenas um modo (i.e., eliminamos a cadeia de Markov) e que contenha $n$ ativos de risco e um outro livre de risco. Esta simplificação leva o nosso modelo a resultados que coincidem com os obtidos por A. Černý [5]. Em seguida, restringimos nosso modelo ao caso em que o mercado opere em um único modo e que contenha apenas um ativo de risco e um outro livre de risco.

No Capítulo 4, apresentamos resultados gerais para o apreçamento e hedging de um derivativo qualquer com característica européia cujo payoff dependa de apenas um ativo de risco. O modelo assumido aqui é o de um mercado que opere em tempo discreto e em apenas um modo, num espaço de estados contínuo. O processo que descreve a dinâmica dos retornos do ativo de risco é um processo de difusão com saltos (jump diffusion process) e com parâmetros constantes (constant investment opportunity set).

No Capítulo 5, aplicamos os resultados gerais obtidos no Capítulo 4 para o caso de uma opção vanilla de compra européia, obtendo expressões explícitas fechadas para o preço desta opção e para o controle necessário à execução do hedging desse derivativo. Em seguida, esses resultados são simplificados ao caso em que o processo que descreve a dinâmica dos retornos do ativo de risco é um movimento browniano (i.e., um processo de Wiener) com parâmetros constantes.

No Capítulo 6, são apresentados os resultados de simulações numéricas feitas para o hedging de uma opção vanilla de compra européia quando os retornos do ativo de risco são descritos por um movimento browniano.

No Capítulo 7 são apresentadas as conclusões desta tese, bem como algumas oportunidades futuras de pesquisa que derivam diretamente do que foi pesquisado e apresentado aqui.

Por fim, os apêndices contêm lemas e teoremas auxiliares, sua demonstração, bem como as demonstrações dos lemas, teoremas e corolários apresentados no corpo da tese.

\subsection{Contribuições Originais}

As contribuições originais desta tese são as seguintes.

1. Resultados apresentados no Capítulo 3, Item 3.1; 
2. Todos os resultados apresentados no Capítulo 4;

3. Todos os resultados apresentados no Capítulo 5.

Os resultados mencionados acima e apresentados no Capítulo 3 generalizam resultados já obtidos em outras pesquisas, tornando-os casos particulares da teoria aqui desenvolvida.

Os resultados apresentados no Capítulo 4 representam um avanço interessante em relação ao modelo desenvolvido no Capítulo 3, pois incorporam elementos concretos e práticos da estrutura de um mercado financeiro real, a saber, a dinâmica dos retornos do ativo de risco descrita segundo um processo de difusão com saltos num espaço de estados contínuo. Esses resultados estabelecem a estrutura teórica a partir da qual se podem obter expressões analíticas fechadas para: (1) o preço de um derivativo com um payoff qualquer (i.e., completamente genérico), desde que de característica européia e com tratabilidade matemática, e (2) o controle ótimo da carteira que faz o hedging de mínima variância desse derivativo, num modelo de mercado em que o tempo é discreto e o espaço de estados é contínuo, atendendo assim às premissas que melhor refletem as condições de funcionamento do mercado financeiro real (conforme já descrito no Item 1.2). No caso de não haver tratabilidade matemática suficiente, impossibilitando a obtenção de expressões analíticas fechadas, essa mesma estrutura apresentada no Capítulo 4 provê os meios para a execução de simulações numéricas de Monte Carlo [17, 12], em todas as suas nuanças, permitindo então obter os mesmos resultados descritos acima, porém de forma numérica.

Os resultados apresentados no Capítulo 5 correspondem à aplicação dos resultados obtidos no Capítulo 4 - onde o modelo adotado para o mercado é discreto com relação ao tempo e contínuo com relação ao espaço de estados a um tipo de derivativo já muito bem conhecido e usado no mercado financeiro: uma opção de compra européia vanilla. Esses resultados são muito interessantes, não só pela originalidade, mas também porque correspondem a um paralelo mais realista e mais correto, do ponto de vista prático, do resultado apresentado por Merton [20], onde o modelo adotado para o mercado é contínuo com relação ao tempo e ao espaço de estados.

Ainda no Capítulo 5, particularizamos os resultados obtidos para a opção de compra vanilla européia (mencionados acima) ao caso em que os retornos do ativo de risco são descritos por um processo de difusão sem saltos, i.e., por um processo browniano (ou de Wiener). Esses resultados também são interessantes, não só pela originalidade, mas também porque correspondem a um paralelo mais realista e mais correto, do ponto de vista prático, do resultado apresentado por Black \& Scholes [3], onde o modelo adotado para o mercado é contínuo com relação ao tempo e ao espaço de estados. 


\section{Capítulo 2}

\section{Revisão Bibliográfica}

Neste capítulo fazemos uma breve revisão bibliográfica do assunto de hedging de mínima variância. No Item 2.1, apresentamos os conceitos básicos necessários para se compreender a formalização matemática do problema. No Item 2.2, apresentamos resumidamente o que já se desenvolveu sobre apreçamento e hedging de derivativos em mercados incompletos, em tempo contínuo, usando-se critérios quadráticos como medida de risco. No Item 2.3, repetimos o que foi feito no item anterior, considerando, agora, o caso em que o tempo é discreto.

\subsection{Conceitos Básicos}

Seja um mercado financeiro com $d+1$ ativos primários (ativos base) disponíveis para negociação. Os preços desses ativos são representados pelos processos estocásticos $S^{i}=\left\{S_{t}^{i}\right\}_{0 \leq t \leq T} \operatorname{com} i=0,1, \cdots, d$. O ativo $S^{0}$ é considerado o ativo livre de risco. Os outros são chamados ativos de risco.

Uma forma natural de abordar questões apresentadas no Item 1.1 é considerar estratégias dinâmicas de portfólio da forma $\left(u, u_{0}\right)=\left(u_{t}, u_{0, t}\right)_{0 \leq t \leq T}$, onde $u$ é um processo $d$-dimensional previsível de componentes $u_{t}^{i}, i=1, \cdots, d$, e $u_{0}$ é um processo adaptado. Nessa estratégia, $u_{t}^{i}$ descreve o número de unidades do ativo $i$ existentes no portfólio no instante de tempo $t$, e $u_{0, t}$ é o montante investido no ativo livre de risco no instante de tempo $t$. A previsibilidade de $u$ é a formalização matemática da restrição informal de que $u$ não pode antecipar movimentos dos ativos base (i.e., não existe informação privilegiada, ou insider trading).

Empreguemos o ativo livre de risco como numerário, i.e., façamos todos os preços dos ativos $S^{i}$ do mercado serem expressos em relação ao preço $S^{0}$ do ativo livre de risco. Assim, todos os preços dos ativos do mercado passam a ser descontados por $S^{0}$, e o ativo livre de risco passa a possuir preço descontado unitário (i.e., igual a 1) em todos os instantes de tempo $t$. Os preços dos outros ativos, $S^{i}$, passam a ser expressos por um vetor $X$ de componentes $X^{i}=\frac{S^{i}}{S^{0}}, i=1, \cdots, d$. Num instante de tempo $t$, o valor do portfólio $\left(u_{t}, u_{0, t}\right)$ é dado por $V_{t}=u_{t} X_{t}+u_{0, t}$, e os ganhos acumulados pela estratégia até o instante de tempo $t$ correspondem a $G_{t}(u)=\int_{0}^{t} u_{s} d X_{s}$. Para que a última expressão seja bem-definida, assumimos que $X$ seja um semi-martingale [25, 26], o que faz, então, $G_{t}(u)$ ser a integral estocástica de $u$ com respeito a $X$. Os custos 
acumulados até o instante de tempo $t$ devidos ao uso da estratégia $\left(u, u_{0}\right)$ são dados pelo processo $C_{t}(u)=V_{t}-\int_{0}^{t} u_{s} d X_{s}=V_{t}-G_{t}(u)$. Uma estratégia é chamada de auto-financiável se o seu processo de custos acumulados $C$ é constante ao longo do tempo, ou, equivalentemente, se o processo que representa o valor $V_{t}$ da estratégia for dado por:

$$
V_{t}=V_{0}+\int_{0}^{t} u_{s} d X_{s}=V_{0}+G_{t}(u)
$$

onde $V_{0}=C_{0}$ é o valor inicial investido no portfólio, i.e., o valor inicial requerido para se iniciar a estratégia. Notemos que uma estratégia auto-financiável é completamente determinada por $V_{0}$ e $u$. De fato, sendo a estratégia autofinanciável, a partir de $V_{0}$ e $u$ é possível se obter $V_{t}$ para qualquer $t$; e, a partir de $V_{t}$ e $u_{t}$ se obtém $u_{0, t}$.

Consideremos um derivativo $H$ e suponhamos que exista uma estratégia auto-financiável $\left(V_{0}, u\right)$ cujo valor terminal $V_{T}$ seja igual a $H_{T}$ com probabilidade 1. Se nosso modelo do mercado financeiro não permitir arbitragem, é evidente que o preço de $H$ no instante de tempo $t=0, H_{0}$, deve ser dado por $V_{0}$, e que $u$ fornece uma estratégia de hedging com relação a $H$. Adicionalmente, pode-se provar que esta estratégia auto-financiável $\left(V_{0}, u\right)$ é única [21]. Esta foi a idéia básica que levou ao modelo de Black \& Scholes [3] para apreçamento de opções. A estrutura matemática desse problema e suas conexões com a teoria de martingales $[34,25,26]$ foram subseqüentemente desenvolvidas e esclarecidas por J. M. Harrison e D. M. Kreps; uma descrição detalhada pode ser encontrada no artigo de J. M. Harrison e S. R. Pliska [14]. De acordo com a terminologia empregada por eles, um derivativo financeiro $H$ é realizável se existir uma estratégia auto-financiável com $V_{T}=H_{T} P$-a.s.. Conforme a Equação (2.1), isso significa que $H_{T}$ pode ser escrito como:

$$
H_{T}=H_{0}+\int_{0}^{T} u_{s}^{H} d X_{s},
$$

i.e., como uma soma de uma constante $H_{0}$ e uma integral estocástica de $u_{s}^{H}$ com respeito a $X$, onde $u_{s}^{H}$ é a estratégia que tem por objetivo replicar o derivativo $H$. O mercado é completo quando todo derivativo financeiro é realizável. A importância do conceito de um mercado completo se deve ao fato de que, numa tal situação, o apreçamento e o hedging de derivativos financeiros podem ser feitos independentemente das preferências dos agentes econômicos que participam do mercado. Contudo, a completude de um modelo de um mercado é uma propriedade bastante delicada que já se destrói mesmo com a introdução de pequenas alterações da estrutura desse mercado. Por exemplo, um modelo em que os ativos se comportam de acordo com um movimento browniano geométrico (modelo clássico considerado por Black \& Scholes) se torna incompleto se a volatilidade for influenciada por um outro fator estocástico, ou se a dinâmica dos preços considerar saltos (descontinuidades). Ainda, se não for possível efetuar rebalanceamentos de uma carteira a intervalos infinitesimais de tempo (o que, evidentemente, é o que ocorre na prática), o modelo também torna-se incompleto.

Numa situação em que o derivativo não é realizável (i.e., o mercado é incompleto), não existe, por definição, um portfólio que replique esse derivativo, e pode-se mostrar que não há um preço único para esse derivativo [23, 21]. Nesse 
caso, se ainda insistirmos em uma abordagem de apreçamento e hedging que independa das preferências dos agentes econômicos, podemos estudar o intervalo de possíveis preços para o derivativo $H$ que são consistentes com ausência de arbitragem em um mercado que possua o ativo de risco, o ativo livre de risco, e o derivativo $H$ como instrumentos financeiros negociados. Uma abordagem alternativa é introduzir critérios subjetivos de acordo com os quais são escolhidas estratégias e os preços dos derivativos são calculados.

Se considerarmos um mercado completo, ou seja, um mercado em que para qualquer instrumento financeiro há uma estratégia que reproduza perfeitamente os seus retornos (chamada estratégia replicante), uma instituição que venda um derivativo qualquer pode eliminar totalmente o seu risco financeiro simplesmente construindo essa estratégia replicante. Por outro lado, se considerarmos um mercado incompleto, onde há instrumentos financeiros para os quais não existe uma estratégia replicante, a redução de risco financeiro pode apenas ocorrer de forma parcial.

Nesse caso, uma estratégia de redução de risco possível é conhecida como hedging de mínima variância, que tem como critério de otimização minimizar o valor esperado do desvio quadrático entre o valor do portfólio gerado pela estratégia de hedging e o valor de mercado $H(T, S(T))$ do contrato derivativo para o qual não há estratégia replicante.

No item a seguir, apresentamos uma breve revisão da literatura acerca de apreçamento e hedging de derivativos em mercados incompletos por meio de critérios quadráticos.

\subsection{Estratégias de Controle (Hedging) com Cri- térios Quadráticos em Tempo Contínuo}

Para um derivativo financeiro não-realizável, é impossível, por definição, encontrar uma estratégia auto financiável com valor terminal $V_{T}=H_{T}$. Uma primeira abordagem possível na busca de uma estratégia de hedging para o derivativo é insistir na condição terminal $V_{T}=H_{T}$. Como é permitido a $u_{0}$ ser um processo adaptado, esta condição terminal $V_{T}=H_{T}$ pode sempre ser atingida pela escolha de $u_{0, T}$. Contudo, como estas estratégias não são, em geral, auto-financiáveis, uma "boa" estratégia pode ser entendida como aquela que tenha um processo de custos acumulados $C$ "pequeno". O emprego de um critério quadrático para medir o risco de uma estratégia foi primeiramente proposto por H. Fölmer e D. Sondermann [11] para o caso em que $X$ é um martingale. M. Schweizer [28] estendeu esse modelo para o caso geral em que $X$ é um semi-martingale. As estratégias derivadas desta abordagem são chamadas de estratégias de mínimo risco local.

Sob algumas hipóteses técnicas [31], estratégias de mínimo risco local podem ser caracterizadas pela seguinte propriedade: seu processo de custos acumulados $C$ deve ser um martingale (de forma que, ainda que a estratégia não seja autofinanciável em todos os instantes de tempo, ela permaneça auto-financiável em média) ortogonal à componente do processo de $X$ que também é um martingale (esta componente de $X$ que é um martingale é usualmente chamada de $M$ ). Aplicando esses conceitos ao derivativo $H$, obtém-se que existe uma estratégia de mínimo risco local para $H$ se, e somente se, $H$ admitir uma decomposição 
da forma:

$$
H_{T}=H_{0}+\int_{0}^{T} u_{s}^{H} d X_{s}+L_{T}^{H} \quad P-a . s .,
$$

onde $L_{T}^{H}$ é um martingale ortogonal a $M$.

A decomposição indicada pela Equação (2.3) tem sido chamada de decomposição de Föllmer-Schweizer de $H$. Ela pode ser vista como uma generalização para o caso semi-martingale da decomposição clássica de Galtchouk-KunitaWatanabe da teoria de martingales. Sua importância em finanças matemáticas reside no fato de ela prover diretamente a estratégia de mínimo risco local para $H$. A componente $u$ relacionada ao ativo base $X$ é dada pelo integrando $u^{H}$, e $u_{0}$ é determinado pela imposição de que o processo de custos acumulados $C$ coincida com $H_{0}+L^{H}$.

Notemos que o caso especial de um derivativo realizável, representado pela Equação (2.2), simplesmente corresponde à ausência do termo ortogonal $L_{T}^{H}$ na decomposição indicada na Equação (2.3). Em casos particulares, é possível encontrar construções mais explícitas para a decomposição indicada na Equação (2.3). No caso de o domínio do tempo ser discreto e finito, $u^{H}$ e $L^{H}$ podem ser computados recursivamente de trás-para-frente no tempo. Se $X$ for um processo contínuo, a decomposição de Föllmer-Schweizer sob a medida de probabilidade $P$ pode ser obtida como uma decomposição de Galtchouk-Kunita-Watanabe computada sob a chamada medida mínima de martingale $\hat{P}$.

Uma desvantagem da abordagem descrita acima se deve ao fato de que é necessário trabalhar com estratégias não auto-financiáveis. Se preferirmos evitar custos intermediários, ou fluxos de caixa não planejados ao longo do tempo, uma segunda abordagem na busca de uma estratégia de hedging para o derivativo consiste em insistir na restrição de que essa estratégia seja auto-financiável (Equação (2.1)). Os possíveis resultados de estratégias como essa são da forma $V_{0}+G_{T}(u)$, onde $V_{0} \in \mathbb{R}$ é um investimento inicial e $u$ pertence ao conjunto $\Theta$ de todos os integrandos permitidos na Equação (2.1). Por definição, um derivativo não-realizável $H$ não pode ser expresso dessa forma e, portanto, parece natural procurar por uma melhor aproximação de $H_{T}$ pelo valor terminal $V_{0}+G_{T}(u)$, gerado por algum par $\left(V_{0}, u\right)$.

O uso de um critério quadrático para medir a qualidade dessa aproximação foi proposto por N. Bouleau e D. Lamberton [4] para o caso em que $X$ é simultaneamente um martingale e uma função de um processo markoviano. Casos mais gerais de $X$ foram tratados por D. Duffie e H. Richardson [9] e por M. Schäl [27], entre outros. As estratégias derivadas dessa abordagem são chamadas de estratégias ótimas de mínima variância. Uma solução geral e completa para o problema foi finalmente obtida por T. Rheinländer e M. Schweizer [24] e por C. Gourieroux, J. P. Laurent e H. Pahm [13].

T. Rheinländer e M. Schweizer [24] descrevem a estrutura das projeções $L^{2}(P)$ que mapeiam uma variável aleatória $\mathcal{F}_{\tau}$-mensurável $H \in L^{2}(P)$ no espaço $G_{T}(\Theta)$ das integrais estocásticas $G(u)=\int u d X$, e provêem o integrando $u^{H} \in \Theta$ resultante desta projeção na forma de uma recursão. Esses conceitos provêem os meios para se encontrarem as estratégias de hedging de mínima variância mencionadas anteriormente, e generalizam resultados obtidos anteriormente que impunham restrições bastante severas em $X$.

C. Gourieroux, J. P. Laurent e H. Pahm [13] consideram o problema de hedging de mínima variância para a situação em que o processo que descreve o 
comportamento dos ativos com risco é um semi-martingale contínuo. Enquanto a abordagem tradicional ao problema trata de portfólios auto-financiáveis envolvendo os ativos primitivos disponíveis no mercado, esses autores introduzem no mercado um numerário como um ativo passível de negociação e mostram como portfólios auto-financiáveis podem ser expressos com relação a esse novo conjunto estendido de ativos, sem alterar o conjunto dos derivativos realizáveis existentes no mercado. O numerário de hedging introduzido é então relacionado à chamada medida de martingale de ótima variância. Usando esse numerário simultaneamente como um deflator e para estender o conjunto primitivo de ativos, os autores transformam o problema original do hedging de mínima variância em um outro equivalente, porém mais simples. O problema de otimização quadrática transformado resultante é então resolvido pelo teorema de projeção de Galtchouk-Kunita-Watanabe sob uma medida de probabilidade de martingale para o conjunto de ativos estendido. Com isso, os autores provêem uma descrição explícita da estratégia ótima de hedging para o problema original de hedging de mínima variância.

J. P. Laurent e H. Pahm [18] usam a estrutura estabelecida por C. Gourieroux, J. P. Laurent e H. Pahm [13] e consideram o problema de hedging de mínima variância quando os preços dos ativos são descritos por processos de Itô [26] em um mercado incompleto. O numerário de hedging e a medida de martingale de ótima variância mostram-se ferramentas fundamentais na determinação estratégia ótima de hedging. Esses autores estudam o numerário de hedging e a medida de martingale de ótima variância em modelos de volatilidade estocástica, e obtêm caracterizações explícitas para eles, usando métodos de programação dinâmica para resolver um problema de controle estocástico.

Arai [1] estende as abordagens de T. Rheinländer e M. Schweizer [24] e de C. Gourieroux, J. P. Laurent e H. Pahm [13] e considera o problema de hedging de mínima variância para modelos em que os preços dos ativos são descontínuos.

Quando os preços dos ativos são descritos por processos de Itô, o valor de um portfólio formado por esses ativos evolui segundo uma equação diferencial estocástica linear, e o problema de hedging de mínima variância pode ser tratado como um problema de controle ótimo linear quadrático (LQ).

Tradicionalmente nos problemas de controle LQ, os parâmetros que descrevem a dinâmica das variáveis envolvidas são considerados determinísticos. Nesta situação, o problema de controle LQ se reduz a resolver a chamada equação diferencial ordinária de Riccati. Se essa equação possuir uma solução positiva definida, pode-se resolver o problema LQ e encontrar o controle e o custo ótimos.

X. Y. Zhou e D. Li [36] tratam o problema de hedging de mínima variância como um problema LQ de parâmetros determinísticos e obtêm sua solução estudando a equação de Riccati associada. Nesse caso, graças à estrutura desse problema de finanças, a equação diferencial ordinária de Riccati se reduz a uma equação linear e sua solução decorre imediatamente da teoria padrão. A. Lim [19] aborda os problemas de apreçamento e de hedging de mínima variância em um mercado incompleto onde os preços dos ativos seguem movimentos brownianos geométricos. Os parâmetros que descrevem o modelo do mercado podem ser processos estocásticos (e.g., volatilidade estocástica). Lim aborda esses problemas sob a perspectiva do controle ótimo linear quadrático e das equações diferenciais estocásticas reversas. Em outras palavras, Lim se concentra na chamada equação de Ricatti estocástica associada aos problemas de apreçamento 
e de hedging de mínima variância, demonstrando a existência e unicidade da solução dessa equação para essa classe de problemas originados de aplicações em finanças. Lim mostra ainda que, como já se poderia esperar, existe uma estreita relação entre a equação de Ricatti estocástica e a medida de martingale de ótima variância.

\subsection{Estratégias de Controle (Hedging) com Cri- térios Quadráticos em Tempo Discreto}

Todos os trabalhos citados acima consideram o problema do hedging de mínima variância em tempo contínuo, i.e., em todos as abordagens mencionadas acima, o tempo é uma variável que assume valores no conjunto dos números reais nãonegativos $\left(t \in \mathbb{R}_{+}\right)$.

Estudos sobre o problema do hedging de mínima variância em tempo discreto (i.e., o tempo é indexado por uma variável que assume valores num conjunto enumerável) são relativamente poucos. M. Schäl [27] aplica métodos de programação dinâmica no caso de um conjunto de oportunidades de investimento constante para analisar o desempenho de diferentes critérios de otimização quadráticos no problema de hedging de opções. M. Schweizer [29] resolve o problema de hedging de mínima variância para um derivativo genérico $H$ num mercado sem friç̧ão composto por um ativo com risco e por taxas de juros não-estocásticas. A solução encontrada pelo autor, entretanto, não desfruta de uma estrutura completamente recursiva. Mais especificamente, a estratégia ótima proposta pelo autor, $\left\{\xi_{k}\right\}_{k=1, \ldots, T}$, é composta por dois processos previsíveis $\left\{\beta_{k}\right\}_{k=1, \ldots, T}$ e $\left\{\rho_{k}\right\}_{k=1, \ldots, T}$ expressos como esperanças condicionais em cada um dos nós do espaço de estados. Esse procedimento é altamente ineficiente do ponto de vista computacional: em um árvore trinomial recombinante, por exemplo, são necessárias $3^{(T-t)}$ operações a cada instante de tempo $t$, para todos os instantes $t$. Na mesma situação, uma solução completamente recursiva obtida por meio de um algoritmo de programação dinâmica requer apenas três operações a cada instante de tempo $t$, para todos os instantes $t$. Essa solução foi encontrada por D. Bertsimas, L. Kogan e A. W. Lo [2] para o caso de um ativo com risco e taxas de juros não-estocásticas. A. Černý [5] propõe uma solução para o problema geral de hedging de mínima variância em tempo e espaço de estados discretos usando métodos de programação dinâmica. As contribuições de seu trabalho são resolver o problema de hedging de mínima variância para um número arbitrário de ativos com risco em ambientes de taxas de juros estocásticas, e propor uma solução simples completamente recursiva que, em modelos markovianos para o mercado, melhoram significativamente a eficiência computacional em comparação aos resultado obtidos por M. Schweizer [29]. 


\section{Capítulo 3}

\section{Modelos em Tempo Discreto}

Nos itens a seguir, apresentamos 3 modelos de mercado financeiro operando em tempo discreto.

O primeiro deles, chamado de "modelo geral", considera que o mercado financeiro pode operar, a cada instante de tempo $\tau$, em $N$ diferentes modos possíveis (por exemplo, mercado "normal", mercado "nervoso"e mercado "calmo"). Além disso, nesse mercado existem $n$ ativos de risco básicos e um ativo livre de risco.

Os dois outros modelos são obtidos a partir de simplificações desse primeiro.

Assim, o segundo modelo, chamado de "modelo com $n$ ativos de risco sem cadeia de Markov", é obtido a partir do primeiro modelo simplesmente adicionandose a restrição de que existe apenas um único modo de operação possível para o mercado a cada instante de tempo $\tau$. Nesse segundo modelo, continuam existindo no mercado $n$ ativos de risco básicos.

O terceiro modelo, chamado de "modelo com 1 ativo de risco sem cadeia de Markov", é obtido a partir do segundo modelo simplesmente adicionando-se a restrição de que existe apenas um ativo de risco básico no mercado, em oposição aos $n$ existentes anteriormente.

\subsection{Modelo Geral}

Neste item consideramos um modelo de mercado financeiro que opere em tempo discreto, em diferentes modos, e que contenha $n+1$ ativos básicos (também chamados de ativos base), sendo um deles livre de risco, e os outros $n$ com risco (também chamados de ativos de risco). Além disso, esse mercado contém também um derivativo não-realizável, de característica européia, cujo payoff depende dos $n$ ativos básicos com risco. Consideramos, ainda, a existência de um portfólio auto-financiável composto por uma combinação dos $n+1$ ativos base.

O propósito deste item é encontrar a estratégia ótima de controle do portfólio, i.e., a composição ótima do portfólio que produza um hedging de mínima variância para o derivativo não-realizável.

No Item 3.1.1 definimos variáveis e elementos matemáticos básicos envolvidos na modelagem do problema de hedging de mínima variância de um contrato derivativo não-replicável. No Item 3.1.2 descrevemos os elementos do modelo, 
estabelecendo a dinâmica, i.e., a equação de estados, do valor do portfólio autofinanciável contendo os $n+1$ ativos base. No Item seguinte, 3.1.3, formulamos o problema de controle ótimo estocástico que descreve o hedging de mínima variância para o derivativo não-realizável. Por fim, no Teorema 3.1 apresentamos a solução desse problema de controle ótimo estocástico.

\subsubsection{Definições}

Abordar o tempo de forma discreta significa admitir que o tempo consiste em uma seqüência ordenada de pontos, i.e., que o tempo $\tau$ é representado por elementos do conjunto $\{\tau \mid \tau \in \mathbb{N}\}$. Esse tipo de abordagem para o tempo é conveniente quando os eventos e suas conseqüências ocorrem, ou são levados em consideração, apenas a intervalos de tempo discretos, e.g., a cada $n$ dias, a cada $n$ semanas, a cada $n$ meses, a cada $n$ anos etc.

Um processo $X$ ser $\mathcal{F}_{\tau}$-mensurável (ou $\mathcal{F}_{\tau}$-adaptado) significa que o valor de $X$ é conhecido no instante de tempo $\tau$ e desconhecido em instantes anteriores a $\tau$.

Um processo $X$ ser previsível em $\tau$ significa que o valor de $X_{\tau}$ já é conhecido em instantes de tempo anteriores a $\tau$, i.e., $X$ é $\mathcal{F}_{\tau^{-}}$-mensurável, onde $\tau^{-}<\tau$.

Um mercado financeiro operando em tempo discreto e em diferentes modos corresponde a um sistema dinâmico que pode ser descrito por um espaço de probabilidade $(\Omega, \mathcal{F}, \mathbb{P})$, um horizonte de tempo $\mathcal{T} \in \mathbb{N}$, uma filtragem $\left\{\mathcal{F}_{\tau}\right\}_{\tau=1, \cdots, \mathcal{T}}$ e uma classe de modelos matemáticos $\mathcal{G}_{i}, i \in\{1, \ldots, N\}$. Admitimos que o número de modos de operação diferentes que o mercado financeiro pode assumir ao longo do tempo seja finito, contável e igual a $N$, e que cada um desses modos de operação possa ser adequadamente descrito por um dos modelos $\mathcal{G}_{i}, i \in\{1, \ldots, N\}$, mencionados anteriormente.

Denotamos os modos de operação do mercado por um processo estocástico $\theta(\tau)$ que assume valores no conjunto $\{i \mid i=1, \ldots, N\}$. Assim, $\theta(\tau)=i$ significa que, no instante de tempo $\tau$, o modo de operação do mercado é adequadamente descrito pelo modelo matemático $\mathcal{G}_{i}$. Admitimos que o processo estocástico $\theta(\tau)$ é $\mathcal{F}_{\tau}$-mensurável, ou seja, que o modo de operação $\theta(\tau)$ do mercado é conhecido a cada instante de tempo $\tau$.

As transições (i.e., os saltos) do mercado financeiro de um modo de operação para outro são causadas por alterações e choques de natureza qualitativa, que admitimos ocorrer possivelmente apenas nos instantes de tempo $\tau=1, \cdots, \mathcal{T}$. Portanto, entre dois instantes de tempo consecutivos, o modo de operação do mercado permanece inalterado.

Admitimos que as transições evoluem estocasticamente segundo uma cadeia de Markov. Assim, dado que num instante de tempo $\tau$ o modo de operação do mercado é $\theta(\tau)=i$, nós sabemos a probabilidade de o mercado, no instante de tempo seguinte, passar a operar em qualquer outro modo admissível, ou mesmo de permanecer no modo atual, e esta probabilidade depende apenas do modo atual de operação. De forma matemática: $P\{\theta(\tau+1)=j \mid \theta(\tau)=i\}=p_{i, j}(\tau)=$ $p_{i, j}$ (suprimimos a dependência explícita do tempo para simplificar a notação).

$E_{\tau}^{P}\{X\}=E^{P}\left\{X \mid \mathcal{F}_{\tau}\right\}$ corresponde ao valor esperado da variável aleatória $X$ com relação à medida de probabilidade $P$, condicionado ao conjunto de informação $\mathcal{F}_{\tau}$ disponível no instante de tempo $\tau$.

$S_{0}\left(\tau, \omega_{\tau} \in \Omega, \theta(\tau-1)\right)$, indicado simplificadamente por $S_{0}(\tau)$ ou por $S_{\theta(\tau-1), 0}(\tau)$, conforme apropriado, é uma variável aleatória previsível que repre- 
senta o valor de mercado (i.e., o preço) no instante de tempo $\tau$, dado que no instante de tempo $\tau-1$ o mercado encontra-se no modo de operação $\theta(\tau-1)$, de um título de renda fixa livre de risco com valor inicial unitário (i.e., $S_{0}(0)=1$ ). Como esse processo é previsível, $S_{0}(\tau)$ já é conhecido no instante de tempo $\tau-1$ (i.e., é $\mathcal{F}_{\tau-1}$-mensurável). Mais especificamente, $E_{\tau-1}^{P}\left\{S_{0}(\tau)\right\}=S_{0}(\tau)$.

$r_{\theta(\tau)}(\tau)$ é uma variável aleatória $\mathcal{F}_{\tau}$-mensurável que representa o retorno, do instante de tempo $\tau$ para o instante de tempo $\tau+1$, de uma unidade monetária aplicada, no instante de tempo $\tau$, no ativo livre de risco quando o mercado encontra-se no modo de operação $\theta(\tau)$. Ou seja, uma unidade monetária aplicada no ativo livre de risco desde o instante de tempo $\tau$ até o instante de tempo $\tau+1$ produz um rendimento, quando o mercado encontra-se no modo de operação $\theta(\tau)$, de $r_{\theta(\tau)}(\tau)$ unidades monetárias no instante de tempo $\tau+1$. É simples ver que $S_{0}(\tau)=\left(1+r_{\theta(0)}(0)\right)\left(1+r_{\theta(1)}(1)\right) \cdots\left(1+r_{\theta(\tau-1)}(\tau-1)\right)$ e que $S_{\theta(\tau), 0}(\tau+1)=S_{0}(\tau)\left(1+r_{\theta(\tau)}(\tau)\right)$.

$S_{l}\left(\tau, \omega_{\tau} \in \Omega, \theta(\tau-1)\right), l=1, \ldots, n$, indicado simplificadamente por $S_{l}(\tau)$ ou por $S_{\theta(\tau-1), l}(\tau)$, conforme apropriado, é uma variável aleatória $\mathcal{F}_{\tau}$-mensurável que representa o preço, no instante de tempo $\tau$, dado que no instante de tempo $\tau-1$ o mercado encontra-se no modo de operação $\theta(\tau-1)$, do $l$-ésimo ativo de risco.

$S_{\theta(\tau-1)}(\tau)$ é um vetor $n \times 1$ cujos elementos são os $S_{\theta(\tau-1), l}(\tau), l=1, \ldots, n$. Portanto, $S_{\theta(\tau-1)}(\tau)$ é um vetor de preços que contém os preços dos ativos de risco $(l=1, \ldots, n)$ :

$$
S_{\theta(\tau-1)}(\tau)=\left(\begin{array}{c}
S_{\theta(\tau-1), 1}(\tau) \\
\vdots \\
S_{\theta(\tau-1), n}(\tau)
\end{array}\right) .
$$

$\xi_{l}\left(\tau, \omega_{\tau} \in \Omega, \theta(\tau-1)\right)$, indicado simplificadamente por $\xi_{l}(\tau)$ ou por $\xi_{\theta(\tau-1), l}(\tau)$, conforme apropriado, é uma variável aleatória $\mathcal{F}_{\tau}$-mensurável que representa os dividendos pagos no instante $\tau$, dado que no instante de tempo $\tau-1$ o mercado encontra-se no modo de operação $\theta(\tau-1)$, para o detentor de uma unidade do $l$-ésimo ativo de risco durante o intervalo de tempo entre os instantes $\tau-1$ e $\tau$.

$\xi_{\theta(\tau-1)}(\tau)$ é um vetor $n \times 1$ cujos elementos são os $\xi_{\theta(\tau-1), l}(\tau+1), l=1, \ldots, n$. Assim:

$$
\xi_{\theta(\tau-1)}(\tau)=\left(\begin{array}{c}
\xi_{\theta(\tau-1), 1}(\tau) \\
\vdots \\
\xi_{\theta(\tau-1), n}(\tau)
\end{array}\right) .
$$

$u_{l}(\tau, S(\tau), \theta(\tau)), l=0, \ldots, n$, indicado simplificadamente por $u_{l}(\tau)$, é uma variável $\mathcal{F}_{\tau}$-mensurável que representa a decisão de controle tomada no instante de tempo $\tau$. Em outras palavras, $u_{l}(\tau)$ corresponde à quantidade de recursos financeiros investida no $l$-ésimo ativo base (de valor $S_{l}(\tau)$ ) do portfólio no instante de tempo $\tau$.

$u(\tau)$ é um vetor cujos elementos são os $u_{l}(\tau), l=1, \ldots, n$. Assim:

$$
u(\tau)=\left(\begin{array}{c}
u_{1}(\tau) \\
\vdots \\
u_{n}(\tau)
\end{array}\right) .
$$

A seqüência $\left\{u_{0}(\tau), u(\tau)\right\}_{\tau=1, \cdots, \mathcal{T}}$ é chamada de política de controle. A política ótima de controle é chamada de estratégia de hedging. 
$V(\tau, S(\tau), u(\tau), \theta(\tau))$, indicado simplificadamente por $V(\tau)$, é uma variável $\mathcal{F}_{\tau}$-mensurável que representa o valor de um portfólio auto-financiável que evolui de acordo com as decisões de controle $\{u(\tau)\}_{\tau=0, \cdots, \mathcal{T}-1}$. Em outras palavras, dizemos que esse portfólio é gerado pela política de controle $\{u(\tau)\}_{\tau=0, \cdots, \mathcal{T}-1}$. No instante de tempo $\tau=0$, o valor do portfólio auto-financiável é $V(0)$.

\subsubsection{Descrição dos Elementos do Modelo Geral}

Consideremos um modelo em que os preços dos $n$ ativos de risco no instante de tempo $\tau$ sejam descritos pelo vetor $S(\tau)$ :

$$
S(\tau)=\left(\begin{array}{c}
S_{1}(\tau) \\
\vdots \\
S_{n}(\tau)
\end{array}\right) .
$$

Suponhamos que, no instante de tempo $\tau$, o mercado encontre-se num modo de operação $\theta(\tau)$ conhecido. Os preços dos $n$ ativos de risco no instante de tempo $\tau+1$, dado que no instante de tempo $\tau$ o mercado encontra-se no modo de operação $\theta(\tau)$, são variáveis aleatórias que descrevemos pelo vetor $S_{\theta(\tau)}(\tau+1)$ :

$$
S_{\theta(\tau)}(\tau+1)=\left(\begin{array}{c}
S_{\theta(\tau), 1}(\tau+1) \\
\vdots \\
S_{\theta(\tau), n}(\tau+1)
\end{array}\right) .
$$

De forma análoga, o preço do ativo livre de risco no instante de tempo $\tau+1$, dado que no instante de tempo $\tau$ o mercado encontra-se no modo de operação $\theta(\tau)$, corresponde a $S_{\theta(\tau), 0}(\tau+1)$, e pode ser escrito como:

$$
S_{\theta(\tau), 0}(\tau+1)=S_{0}(\tau)\left(1+r_{\theta(\tau)}(\tau)\right) .
$$

Seja um portfólio auto-financiável composto por $n+1$ instrumentos financeiros: $n$ ativos de risco e um ativo livre de risco. Suponhamos que, no instante de tempo $\tau$, o portfólio possua valor $V(\tau)$ e contenha $u_{l}(\tau), l=1, \ldots, n$, unidades do $l$-ésimo ativo com risco. Dessa forma, no instante de tempo $\tau$, a composição total do portfólio é tal que $\sum_{l=1}^{n} u_{l}(\tau) S_{l}(\tau)=u^{*}(\tau) S(\tau)$ corresponde ao valor financeiro aplicado nos ativos com risco, e $\left(V(\tau)-u^{*}(\tau) S(\tau)\right)$ corresponde ao valor $u_{0}(\tau)$ aplicado no ativo livre de risco. No instante de tempo $\tau+1$, o valor $V(\tau+1)$ do portfólio auto-financiável passa a ser expresso por:

$$
\begin{aligned}
& V(\tau+1)=u^{*}(\tau)\left(S_{\theta(\tau)}(\tau+1)+\xi_{\theta(\tau)}(\tau+1)\right)+ \\
& \quad\left(V(\tau)-u^{*}(\tau) S(\tau)\right)\left(1+r_{\theta(\tau)}(\tau)\right) .
\end{aligned}
$$

Essa equação se justifica pois as $u(\tau)$ unidades dos ativos de risco presentes no portfólio, incialmente de valor total $u^{*}(\tau) S(\tau)$, passam a valer $u^{*}(\tau)\left(S_{\theta(\tau)}(\tau+\right.$ $\left.1)+\xi_{\theta(\tau)}(\tau+1)\right)$ no instante de tempo $\tau+1$ (evidentemente, já considerando os dividendos pagos pelos ativos de risco). A parcela de valor $\left(V(\tau)-u^{*}(\tau) S(\tau)\right)$, aplicada no ativo livre de risco desde o instante de tempo $\tau$ até o instante de tempo $\tau+1$, se transforma em $\left(V(\tau)-u^{*}(\tau) S(\tau)\right)\left(1+r_{\theta(\tau)}(\tau)\right)$. 
A Equação (3.7) pode ser rescrita como:

$$
\begin{aligned}
V(\tau+1)= & \left(1+r_{\theta(\tau)}(\tau)\right) V(\tau)+ \\
& u^{*}(\tau)\left[S_{\theta(\tau)}(\tau+1)+\xi_{\theta(\tau)}(\tau+1)-S(\tau)\left(1+r_{\theta(\tau)}(\tau)\right)\right] .
\end{aligned}
$$

Lembrando que $S_{\theta(\tau), 0}(\tau+1)=S_{0}(\tau)\left(1+r_{\theta(\tau)}(\tau)\right)$ e definindo:

$$
\begin{gathered}
a_{\theta(\tau)}(\tau)=1+r_{\theta(\tau)}(\tau), \\
\Delta X_{\theta(\tau)}(\tau+1)=\frac{S_{\theta(\tau)}(\tau+1)+\xi_{\theta(\tau)}(\tau+1)}{S_{\theta(\tau), 0}(\tau+1)}-\frac{S(\tau)}{S_{0}(\tau)},
\end{gathered}
$$

a Expressão (3.8), valor do portfólio auto-financiável, pode ser expressa como:

$$
V(\tau+1)=a_{\theta(\tau)}(\tau) V(\tau)+S_{\theta(\tau), 0}(\tau+1) \Delta X_{\theta(\tau)}^{*}(\tau+1) u(\tau) .
$$

$\Delta X_{\theta(\tau)}(\tau+1)$, tal como definido na Equação (3.10), é chamado de processo de ganhos descontado dos ativos de risco.

Apenas para referência futura, definamos $G(\tau+1)$, chamado de processo de ganhos descontado do portfólio auto-financiável. $G(\tau+1)$ é dado por:

$$
\begin{aligned}
& G(\tau+1)=\frac{V(\tau+1)}{S_{\theta(\tau), 0}(\tau+1)}-\frac{V(\tau)}{S_{0}(\tau)}= \\
& \frac{a_{\theta(\tau)}(\tau) V(\tau)+S_{\theta(\tau), 0}(\tau+1) \Delta X_{\theta(\tau)}^{*}(\tau+1) u(\tau)}{S_{\theta(\tau), 0}(\tau+1)}-\frac{V(\tau)}{S_{0}(\tau)}= \\
& \frac{a_{\theta(\tau)}(\tau)}{S_{\theta(\tau), 0}(\tau+1)} V(\tau)+\frac{S_{\theta(\tau), 0}(\tau+1)}{S_{\theta(\tau), 0}(\tau+1)} \Delta X_{\theta(\tau)}^{*}(\tau+1) u(\tau)-\frac{V(\tau)}{S_{0}(\tau)} .
\end{aligned}
$$

Usando $S_{\theta(\tau), 0}(\tau+1)=S_{0}(\tau) a_{\theta(\tau)}(\tau)$, obtemos:

$$
G(\tau+1)=\Delta X_{\theta(\tau)}^{*}(\tau+1) u(\tau) .
$$

\subsubsection{Formulação do Problema de Hedging de Mínima Va- riância}

Denotemos por $\mathcal{F}_{\tau}$ a $\sigma$-álgebra gerada por $\{S(0), \theta(0), \ldots, S(\tau), \theta(\tau)\}$, e definamos:

$$
u_{\tau}=\{u(\tau), \ldots, u(\mathcal{T}-1)\}, \text { onde } u(s) \text { é } \mathcal{F}_{s}-\text { mensurável. }
$$

Consideremos um derivativo não-realizável cujo valor no instante do seu vencimento $\tau=\mathcal{T}$ seja especificado em contrato e representado por uma função $\mathcal{F}_{\mathcal{T}}$-mensurável $H_{\theta(\mathcal{T})}(\mathcal{T})$.

Queremos encontrar uma solução para o problema geral de hedging de mínima variância em tempo discreto, i.e., queremos encontrar uma política ótima de controle que minimize, em $\tau=0$, o valor esperado do desvio quadrático entre o valor terminal $V(\mathcal{T})$ do portfólio gerado por esta política ótima (i.e., pela estratégia de hedging) e o valor terminal $H_{\theta(\mathcal{T})}(\mathcal{T})$ do derivativo não-realizável. 
Em outras palavras, queremos encontrar uma política ótima que minimize o erro de hedging esperado quadrático em $\tau=0$.

O índice de desempenho (ou índice de performance) $\mathcal{J}(\cdot)$ a ser minimizado (i.e., o erro de hedging esperado quadrático em $\tau=0$ ) é dado pelo seguinte funcional:

$$
\begin{gathered}
\mathcal{J}\left(V(\tau=0), \theta(\tau=0),\{u(\tau)\}_{\tau=0, \cdots, \mathcal{T}-1}, \tau=0\right) \\
=\mathcal{J}\left(V(0), \theta(0),\{u(\tau)\}_{\tau=0, \cdots, \mathcal{T}-1}, 0\right) \\
=E_{0}^{P}\left\{\left(V(\mathcal{T})-H_{\theta(\mathcal{T})}(\mathcal{T})\right)^{2}\right\}
\end{gathered}
$$

onde $V(\mathcal{T})$ é o valor terminal do portfólio gerado pela política de controle $\{u(\tau)\}_{\tau=0, \cdots, \mathcal{T}-1}, V(0)$ é o valor inicial investido para criar o portfólio e $H_{\theta(\mathcal{T})}(\mathcal{T})$ é valor terminal do derivativo não-realizável.

Portanto, o problema de hedging de mínima variância de um contrato derivativo não-realizável pode ser expresso como um problema de controle ótimo estocástico em tempo discreto e com variáveis de controle $V(0)$ e $\{u(\tau)\}_{\tau=0, \cdots, \mathcal{T}-1}$, dado por:

$$
\overline{\mathcal{J}}_{0, \mathcal{T}}(\theta(0), 0)=_{V(0),\{u(0), \ldots, u(\mathcal{T}-1)\} \in u_{0}} E^{P}\left\{\left(V(\mathcal{T})-H_{\theta(\mathcal{T})}(\mathcal{T})\right)^{2} \mid \mathcal{F}_{0}\right\} .
$$

A solução desse problema é feita em duas etapas. Primeiramente, consideramos o seguinte problema de controle ótimo estocástico:

$$
\begin{aligned}
\overline{\mathcal{J}}(V(\tau=0), \theta(\tau=0), \tau=0)=\overline{\mathcal{J}}(V(0), \theta(0), 0)= \\
\inf _{\{u(0), \ldots, u(\mathcal{\mathcal { T }}-1)\} \in u_{\tau}} E^{P}\left(\left(V(\mathcal{T})-H_{\theta(\mathcal{T})}(\mathcal{T})\right)^{2} \mid \mathcal{F}_{\tau}\right) .
\end{aligned}
$$

A solução desse problema, ou seja, os $\{\bar{u}(0), \ldots, \bar{u}(\mathcal{T}-1)\} \in u_{0}$, provê a estratégia ótima de controle do portfólio, i.e., a composição ótima do portfólio que produz um hedging de mínima variância para o derivativo não-realizável. Em seguida, tendo sido obtidos os $\{\bar{u}(0), \ldots, \bar{u}(\mathcal{T}-1)\} \in u_{0}$, encontramos $\bar{V}(0)$, que é o valor de $V(0)$ que minimiza $\overline{\mathcal{J}}(V(0), \theta(0), 0)$; i.e., encontramos $\overline{\mathcal{J}}_{0, \mathcal{T}}(\theta(0), 0)=\overline{\mathcal{J}}(\bar{V}(0), \theta(0), 0)$.

Os teoremas apresentados a seguir provêem a solução para o problema dado pela Equação (3.16).

Teorema 3.1. Seja o problema definido pela Equação (3.17). Então, para cada $\tau=\mathcal{T}, \ldots, 0$, a estratégia ótima de controle do portfólio $\bar{u}(\tau)$ e a função objetivo ótima $\overline{\mathcal{J}}(V(\tau), \theta(\tau), \tau)$ são dadas por:

$$
\begin{aligned}
& \bar{u}(\tau)= \\
& -M_{\theta(\tau)}^{-1} \sum_{j} p_{\theta(\tau), j} E_{\tau}^{P}\left\{k_{j}(\tau+1) \Delta X_{\theta(\tau)}(\tau+1)\left(\frac{V(\tau)}{S_{0}(\tau)}-\frac{H_{j}(\tau+1)}{S_{\theta(\tau), 0}(\tau+1)}\right)\right\}= \\
& -M_{\theta(\tau)}^{-1} E_{\tau}^{P}\left\{k_{\theta(\tau+1)}(\tau+1) \Delta X_{\theta(\tau)}(\tau+1)\left(\frac{V(\tau)}{S_{0}(\tau)}-\frac{H_{\theta(\tau+1)}(\tau+1)}{S_{\theta(\tau), 0}(\tau+1)}\right)\right\} \\
& \overline{\mathcal{J}}(V(\tau), \theta(\tau), \tau)=k_{\theta(\tau)}(\tau)\left(V(\tau)-H_{\theta(\tau)}(\tau)\right)^{2}+\delta_{\theta(\tau)}(\tau),
\end{aligned}
$$


onde, para $\theta(\tau)=1, \ldots, N, \tau=\mathcal{T}-1, \ldots, 0$ :

$$
\begin{aligned}
& k_{\theta(\tau)}(\tau)=a_{\theta(\tau)}(\tau)^{2}\left[E_{\tau}^{P}\left\{k_{\theta(\tau+1)}(\tau+1)\right\}-\right. \\
& E_{\tau}^{P}\left(k_{\theta(\tau+1)}(\tau+1) \Delta X_{\theta(\tau)}^{*}(\tau+1)\right) M_{\theta(\tau)}^{-1} \\
& \left.E_{\tau}^{P}\left(k_{\theta(\tau+1)}(\tau+1) \Delta X_{\theta(\tau)}(\tau+1)\right)\right],
\end{aligned}
$$

$$
\begin{array}{r}
H_{\theta(\tau)}(\tau)=\frac{1}{\frac{k_{\theta(\tau)}(\tau)}{a_{\theta(\tau)}(\tau)^{2}}} \\
E_{\tau}^{P}\left\{\left[k_{\theta(\tau+1)}(\tau+1)-E_{\tau}^{P}\left[k_{\theta(\tau+1)}(\tau+1) \Delta X_{\theta(\tau)}^{*}(\tau+1)\right]\right.\right. \\
\left.\left.\quad M_{\theta(\tau)}^{-1} k_{\theta(\tau+1)}(\tau+1) \Delta X_{\theta(\tau)}(\tau+1)\right] \frac{H_{\theta(\tau+1)}(\tau+1)}{a_{\theta(\tau)}(\tau)}\right\},
\end{array}
$$$$
\delta_{\theta(\tau)}(\tau)=E_{\tau}^{P}\left\{k_{\theta(\tau+1)}(\tau+1)\right.
$$$$
\left(a_{\theta(\tau)}(\tau) H_{\theta(\tau)}(\tau)+S_{\theta(\tau), 0}(\tau+1) \Delta X_{\theta(\tau)}^{*}(\tau+1) u(\tau)-\right.
$$$$
\left.\left.H_{\theta(\tau+1)}(\tau+1)\right)^{2}\right\}+E_{\tau}^{P}\left(\delta_{\theta(\tau+1)}(\tau+1)\right) \geq 0,
$$$$
M_{\theta(\tau)}=E_{\tau}^{P}\left\{k_{\theta(\tau+1)}(\tau+1) \Delta X_{\theta(\tau)}(\tau+1) \Delta X_{\theta(\tau)}^{*}(\tau+1)\right\}
$$$$
u(\tau)=-\left[E_{\tau}^{P}\left\{k_{\theta(\tau+1)}(\tau+1) \Delta X_{\theta(\tau)}(\tau+1) \Delta X_{\theta(\tau)}^{*}(\tau+1)\right\}\right]^{-1}
$$$$
E_{\tau}^{P}\left\{k_{\theta(\tau+1)}(\tau+1) \Delta X_{\theta(\tau)}(\tau+1)\left(\frac{H_{\theta(\tau)}(\tau)}{S_{0}(\tau)}-\frac{H_{\theta(\tau+1)}(\tau+1)}{S_{\theta(\tau), 0}(\tau+1)}\right)\right\},
$$

$e$ as condições finais são:

$$
k_{\theta(\mathcal{T})}(\mathcal{T})=1, \quad \delta_{\theta(\mathcal{T})}(\mathcal{T})=0 .
$$

Prova: ver Apêndice A.

Teorema 3.2. Seja $\overline{\mathcal{J}}(V(\tau), \theta(\tau), \tau)$ tal como definido no Teorema 3.1. O valor de $V(0)$ que minimiza $\overline{\mathcal{J}}(V(0), \theta(0), 0)$, denominado por $\bar{V}(0)$, é dado por:

$$
\bar{V}(0)=H_{\theta(0)}(0) \text {. }
$$

Prova: ver Apêndice A. 
Teorema 3.3. Nas condições dos Teoremas 3.1 e 3.2, o erro quadrático de hedging esperado em $\tau=0, \delta_{\theta(0)}(0)$, é dado por

$$
\delta_{\theta(0)}(0)=\overline{\mathcal{J}}_{0, \mathcal{T}}(\theta(0), 0) .
$$

Prova: ver Apêndice A.

Os Teoremas 3.1, 3.2 e 3.3 provêem uma caracterização completa do problema de hedging de mínima variância. De fato, aplicamos o Teorema 3.1 de forma recursiva, partindo de $\tau=\mathcal{T}$ e usando $k_{\theta(\mathcal{T})}(\mathcal{T})=1$ e $\delta_{\theta(\mathcal{T})}(\mathcal{T})=0$, para obter $M_{\theta(\tau)}, k_{\theta(\tau)}(\tau), H_{\theta(\tau)}(\tau), u(\tau)$ e $\delta_{\theta(\tau)}(\tau)$ para $\tau=\mathcal{T}-1, \ldots, 0$. Ao final desse procedimento, i.e., em $\tau=0$, aplicamos o Teorema 3.2, que mostra que $\bar{V}(0)$, valor inicial investido para criar o portfólio, é igual a $H_{\theta(0)}(0)$. Além disso, pelo Teorema 3.3 , obtemos $\overline{\mathcal{J}}_{0, \mathcal{T}}(\theta(0), 0)=\delta_{\theta(0)}(0)$, que corresponde ao erro quadrático de hedging esperado em $\tau=0$.

Em seguida, num procedimento recursivo partindo de $\tau=0$ e indo até $\tau=\mathcal{T}$, podemos obter os $\{\bar{u}(\tau), \ldots, \bar{u}(\mathcal{T}-1)\} \in u_{\tau}$, que provêem a estratégia ótima de controle do portfólio, i.e., a composição ótima do portfólio que produz um hedging de mínima variância para o derivativo não-realizável. Evidentemente, conhecida essa estratégia ótima de controle, também podemos obter pela Equação (3.11), desde $\tau=0$ até $\tau=\mathcal{T}$, o valor $V(\tau)$ do portfólio auto-financiável gerado pela estratégia ótima de controle $\{\bar{u}(\tau)\}_{\tau=0, \cdots, \mathcal{T}-1}$.

\subsubsection{Variance Optimal Measure}

Consideremos o Teorema 3.1. Usando a Equação (A.66), e lembrando que $\frac{k_{\theta(\tau)}(\tau)}{a_{\theta(\tau)}(\tau)^{2}}$ é $\mathcal{F}_{\tau}$-mensurável, podemos escrever $H_{\theta(\tau)}(\tau)$ (Equação (3.21)) como:

$$
H_{\theta(\tau)}(\tau)=E_{\tau}^{P}\left\{m_{\tau+1, \tau}^{P \rightarrow Q}(\theta(\tau+1)) \frac{H_{\theta(\tau+1)}(\tau+1)}{a_{\theta(\tau)}(\tau)}\right\}
$$

onde:

$$
m_{\tau+1, \tau}^{P \rightarrow Q}(\theta(\tau+1))=\frac{A}{B}
$$

$$
\begin{aligned}
& A=k_{\theta(\tau+1)}(\tau+1)- \\
& E_{\tau}^{P}\left[k_{\theta(\tau+1)}(\tau+1) \Delta X_{\theta(\tau)}^{*}(\tau+1)\right] M_{\theta(\tau)}^{-1} k_{\theta(\tau+1)}(\tau+1) \Delta X_{\theta(\tau)}(\tau+1), \\
& B=E_{\tau}^{P}\left\{k_{\theta(\tau+1)}(\tau+1)\right\}- \\
& E_{\tau}^{P}\left[k_{\theta(\tau+1)}(\tau+1) \Delta X_{\theta(\tau)}^{*}(\tau+1)\right] M_{\theta(\tau)}^{-1} E_{\tau}^{P}\left[k_{\theta(\tau+1)}(\tau+1) \Delta X_{\theta(\tau)}(\tau+1)\right],
\end{aligned}
$$

$$
M_{\theta(\tau)}=E_{\tau}^{P}\left\{k_{\theta(\tau+1)}(\tau+1) \Delta X_{\theta(\tau)}(\tau+1) \Delta X_{\theta(\tau)}^{*}(\tau+1)\right\} .
$$

Usando as Equações (3.6) e (3.9), podemos rescrever a Equação (3.28) como:

$$
H_{\theta(\tau)}(\tau)=E_{\tau}^{P}\left\{m_{\tau+1, \tau}^{P \rightarrow Q}(\theta(\tau+1)) \frac{H_{\theta(\tau+1)}(\tau+1)}{\left(\frac{S_{\theta(\tau), 0}(\tau+1)}{S_{0}(\tau)}\right)}\right\} .
$$


Portanto:

$$
\frac{H_{\theta(\tau)}(\tau)}{S_{0}(\tau)}=E_{\tau}^{P}\left\{m_{\tau+1, \tau}^{P \rightarrow Q}(\theta(\tau+1)) \frac{H_{\theta(\tau+1)}(\tau+1)}{S_{\theta(\tau), 0}(\tau+1)}\right\} .
$$

Notemos que $E_{\tau}^{P}\left\{m_{\tau+1, \tau}^{P \rightarrow Q}(\theta(\tau+1))\right\}=1$, e que $m_{\tau+1, \tau}^{P \rightarrow Q}(\theta(\tau+1))$ é $\mathcal{F}_{\tau+1^{-}}$ mensurável. Portanto, a Equação $m_{\tau+1, \tau}^{P \rightarrow Q}(\theta(\tau+1))$ pode ser interpretada como uma mudança de medida de probabilidade da medida $P$ para a medida $Q$ (i.e., a derivada de Radon-Nikodým, $\frac{d Q}{d P}$, da medida $Q$ com relação à medida $P$ ), e a Equação (3.34) pode ser rescrita simplesmente como:

$$
\frac{H_{\theta(\tau)}(\tau)}{S_{0}(\tau)}=E_{\tau}^{Q}\left\{\frac{H_{\theta(\tau+1)}(\tau+1)}{S_{\theta(\tau), 0}(\tau+1)}\right\}
$$

Observemos ainda que, com relação à medida de probabilidade $Q$, a variável aleatória $\frac{H_{\theta(\tau+1)}(\tau+1)}{S_{\theta(\tau), 0}(\tau+1)}$ comporta-se como um martingale dado o conjunto de informações $\mathcal{F}_{\tau}$, i.e., $E_{\tau}^{Q}\left\{\frac{H_{\theta(\tau+1)}(\tau+1)}{S_{\theta(\tau), 0}(\tau+1)}\right\}=\frac{H_{\theta(\tau)}(\tau)}{S_{0}(\tau)}$. Portanto, a medida $Q$ é uma medida de martingale.

Também é interessante notar que, com relação a essa medida, o processo que representa os preços descontados dos ativos, $\frac{S(\tau)}{S_{0}(\tau)}$, bem como o processo que representa o valor descontado do portfólio $\frac{V(\tau)}{S_{0}(\tau)}$, comportam-se como martingales, pois o processo de ganhos descontado dos ativos de risco, $\Delta X_{\theta(\tau)}(\tau+1)$ (Equação (3.10)), bem como o processo de ganhos descontado do portfólio, $G(\tau+1)$ (Equação (3.13)), possuem esperança nula. De fato, para $\Delta X_{\theta(\tau)}(\tau+1)$ temos:

$$
E_{\tau}^{Q}\left\{\Delta X_{\theta(\tau)}^{*}(\tau+1)\right\}=E_{\tau}^{P}\left\{m_{\tau+1, \tau}^{P \rightarrow Q}(\theta(\tau+1)) \Delta X_{\theta(\tau)}^{*}(\tau+1)\right\}=E_{\tau}^{P}\left\{\frac{A}{B}\right\}
$$

onde:

$$
\begin{gathered}
A=E_{\tau}^{P}\left\{k_{\theta(\tau+1)}(\tau+1) \Delta X_{\theta(\tau)}^{*}(\tau+1)\right\}- \\
E_{\tau}^{P}\left\{k_{\theta(\tau+1)}(\tau+1) \Delta X_{\theta(\tau)}^{*}(\tau+1)\right\} M_{\theta(\tau)}^{-1} \\
E_{\tau}^{P}\left\{k_{\theta(\tau+1)}(\tau+1) \Delta X_{\theta(\tau)}(\tau+1) \Delta X_{\theta(\tau)}^{*}(\tau+1)\right\}, \\
B=E_{\tau}^{P}\left\{k_{\theta(\tau+1)}(\tau+1)\right\}- \\
E_{\tau}^{P}\left(k_{\theta(\tau+1)}(\tau+1) \Delta X_{\theta(\tau)}^{*}(\tau+1)\right) M_{\theta(\tau)}^{-1} E_{\tau}^{P}\left(k_{\theta(\tau+1)}(\tau+1) \Delta X_{\theta(\tau)}(\tau+1)\right) .
\end{gathered}
$$

Usando a Equação (3.32), obtemos:

$$
E_{\tau}^{Q}\left\{\Delta X_{\theta(\tau)}^{*}(\tau+1)\right\}=0
$$


Para $G(\tau+1)$, temos:

$$
\begin{aligned}
E_{\tau}^{Q}\{G(\tau+1)\}=E_{\tau}^{Q}\left\{\Delta X_{\theta(\tau)}^{*}(\tau+1) u(\tau)\right\}= \\
E_{\tau}^{P}\left\{m_{\tau+1, \tau}^{P \rightarrow Q}(\theta(\tau+1)) \Delta X_{\theta(\tau)}^{*}(\tau+1) u(\tau)\right\} .
\end{aligned}
$$

Como $u(\tau)$ é $\mathcal{F}_{\tau}$-mensurável, obtemos:

$$
\begin{aligned}
& E_{\tau}^{Q}\{G(\tau+1)\}=E_{\tau}^{P}\left\{m_{\tau+1, \tau}^{P \rightarrow Q}(\theta(\tau+1)) \Delta X_{\theta(\tau)}^{*}(\tau+1)\right\} u(\tau)= \\
& E_{\tau}^{Q}\left\{\Delta X_{\theta(\tau)}^{*}(\tau+1)\right\} u(\tau)=0 .
\end{aligned}
$$

Essa medida de martingale $Q$ que emerge da solução do problema de controle ótimo é chamada de variance optimal measure, e suas propriedades foram estudadas e demonstradas por M. Schweizer [30].

A mudança de medida de probabilidade $m_{\tau+1, \tau}^{P \rightarrow Q}(\theta(\tau+1))$ não é necessariamente estritamente positiva para quaisquer valores de $k_{\theta(\tau+1)}(\tau+1)$ e de $\Delta X_{\theta(\tau)}(\tau+1)$, ou seja, $Q$ é uma medida com sinal e não necessariamente equivalente à medida $P$. Entretanto, como estamos admitindo um mercado livre de arbitragem, $m_{\tau+1, \tau}^{P \rightarrow Q}(\theta(\tau+1))$ é uma mudança de medida de bem-definida, o que assegura que o operador matemático esperança condicional seja bem-definido com relação à medida de martingale $Q$.

\subsubsection{Cálculo de $H(\tau)$}

A Equação (3.28) permite calcular o valor do contrato derivativo no instante de tempo $\tau$ dado o valor do derivativo no instante de tempo $\tau+1$. Portanto, a partir de $H_{\theta(\mathcal{T})}(\mathcal{T})$, pode-se, por meio de recursões sucessivas, calcular o valor $H_{\theta(\tau)}(\tau)$. O teorema a seguir permite calcular $H_{\theta(\tau)}(\tau)$ diretamente a partir de $H_{\theta(\mathcal{T})}(\mathcal{T})$, sem necessidade de um processo recursivo.

Teorema 3.4. Seja:

$$
H_{\theta(\tau)}(\tau)=E_{\tau}^{P}\left\{m_{\tau+1, \tau}^{P \rightarrow Q}(\theta(\tau+1)) \frac{H_{\theta(\tau+1)}(\tau+1)}{a_{\theta(\tau)}(\tau)}\right\} .
$$

Então:

$$
H_{\theta(\tau)}(\tau)=E_{\tau}^{P}\left\{m_{\mathcal{T}, \tau}^{P \rightarrow Q} \frac{H_{\theta(\mathcal{T})}(\mathcal{T})}{\prod_{j=\tau}^{\mathcal{T}-1} a_{\theta(j)}(j)}\right\}=E_{\tau}^{Q}\left\{\frac{H_{\theta(\mathcal{T})}(\mathcal{T})}{\prod_{j=\tau}^{\mathcal{T}-1} a_{\theta(j)}(j)}\right\}
$$

onde $m_{\mathcal{T}, \tau}^{P \rightarrow Q}$, dado por:

$$
m_{\mathcal{T}, \tau}^{P \rightarrow Q}=\prod_{j=\tau}^{\mathcal{T}-1} m_{j+1, j}^{P \rightarrow Q}(\theta(j+1))
$$

corresponde à mudança de medida de probabilidade da medida P para a medida $Q$.

Prova: ver Apêndice A. 


\subsubsection{Uma Interpretação Para $H(\tau)$}

Num mercado financeiro livre de arbitragem e completo, qualquer instrumento financeiro (e.g., um contrato derivativo) é realizável e possui um preço único correspondente, conforme justificado por argumentos de não-arbitragem, ao valor da estratégia auto-financiável que o replica. Num mercado financeiro livre de arbitragem e incompleto, por outro lado, existem instrumentos financeiros nãorealizáveis, i.e., instrumentos para os quais não há uma estratégia replicante. Para esses instrumentos, não é possível determinarmos um preço empregando argumentos de não-arbitragem envolvendo uma carteira replicante (pois ela não existe). Esses instrumentos não-realizáveis não possuem um preço único, mas sim um conjunto de preços possíveis consistentes com ausência de arbitragem.

Como a medida de probabilidade $Q$ é uma medida de martingale, a Equação (3.43) assemelha-se a uma equação de apreçamento em condições de ausência de arbitragem num mercado completo. M. Schäl [27] refere-se a $H(\tau)$ nesta equação como um "preço justo de hedging"(i.e., fair hedging price) do instrumento financeiro não-realizável de valor terminal $H_{\theta(\mathcal{T})}(\mathcal{T})$. M. Schweizer [29] corretamente admite, contudo, que o termo "preço"pode ser enganoso, pois $H(\tau)$ não necessariamente corresponde a um preço livre de arbitragem para o instrumento financeiro não-realizável de valor terminal $H_{\theta(\mathcal{T})}(\mathcal{T})$. Isso ocorre porque a $v a$ riance optimal measure existe mesmo quando há arbitragem entre os ativos do mercado. A. Černý [5] provê um exemplo simples que ilustra esta situação.

\subsection{Modelo com $n$ Ativos de Risco sem Cadeia de Markov}

Neste item simplificamos o modelo geral apresentado anteriormente. Supomos agora que exista apenas um único modo de operação possível para o mercado a cada instante de tempo $\tau$. O número de ativos de risco básicos no mercado continua sendo igual a $n$.

É importante notarmos que esta simplificação leva o nosso modelo a resultados que coincidem com os obtidos por A. Černý [5].

Portanto, eliminando a cadeia de Markov no modelo geral, i.e., fazendo $\theta(\tau)=1, \tau=\mathcal{T}, \ldots, 0$, os Teoremas $3.1,3.2$ e 3.4 podem ser rescritos como:

Corolário 3.5. Para cada $\tau=\mathcal{T}, \ldots, 0$, a estratégia ótima de controle do portfólio $\bar{u}(\tau)$ e a função objetivo ótima $\overline{\mathcal{J}}(V(\tau), \tau)$ são dadas por:

$$
\begin{gathered}
\bar{u}(\tau)=-M^{-1} E_{\tau}^{P}\left\{k(\tau+1) \Delta X(\tau+1)\left(\frac{V(\tau)}{S_{0}(\tau)}-\frac{H(\tau+1)}{S_{0}(\tau+1)}\right)\right\}, \\
\overline{\mathcal{J}}(V(\tau), \tau)=k(\tau)(V(\tau)-H(\tau))^{2}+\delta(\tau)
\end{gathered}
$$

onde, para $\tau=\mathcal{T}-1, \ldots, 0$ :

$$
\begin{aligned}
k(\tau)=a(\tau)^{2}\left[E_{\tau}^{P}\{k(\tau+1)\}-E_{\tau}^{P}\left(k(\tau+1) \Delta X^{*}(\tau+1)\right) M^{-1}\right. & \\
& \left.E_{\tau}^{P}(k(\tau+1) \Delta X(\tau+1))\right],
\end{aligned}
$$




$$
\begin{gathered}
H(\tau)=\frac{1}{\frac{k(\tau)}{a(\tau)^{2}}}\left(E _ { \tau } ^ { P } \left\{\left[k(\tau+1)-E_{\tau}^{P}\left[k(\tau+1) \Delta X^{*}(\tau+1)\right]\right.\right.\right. \\
\left.\left.\left.M^{-1} k(\tau+1) \Delta X(\tau+1)\right] \frac{H(\tau+1)}{a(\tau)}\right\}\right), \\
\delta(\tau)=E_{\tau}^{P}\left\{k ( \tau + 1 ) \left(a(\tau) H(\tau)+S_{0}(\tau+1) \Delta X^{*}(\tau+1) u(\tau)-\right.\right. \\
\left.H(\tau+1))^{2}\right\}+E_{\tau}^{P}(\delta(\tau+1)) \geq 0, \\
M=E_{\tau}^{P}\left\{k(\tau+1) \Delta X(\tau+1) \Delta X^{*}(\tau+1)\right\} \\
u(\tau)=-\left[E_{\tau}^{P}\left\{k(\tau+1) \Delta X(\tau+1) \Delta X^{*}(\tau+1)\right\}\right]^{-1} \\
E_{\tau}^{P}\left\{k(\tau+1) \Delta X(\tau+1)\left(\frac{H(\tau)}{S_{0}(\tau)}-\frac{H(\tau+1)}{S_{0}(\tau+1)}\right)\right\}
\end{gathered}
$$

e as condições finais são:

$$
k(\mathcal{T})=1, \quad \delta(\mathcal{T})=0
$$

Prova: ver Apêndice A.

Corolário 3.6. Seja $\overline{\mathcal{J}}(V(0), \theta(0), 0)$ tal como definido no Corolário 3.5. O valor de $V(0)$ que minimiza $\overline{\mathcal{J}}(V(0), \theta(0), 0)$, denominado por $\bar{V}(0)$, é dado por:

$$
\bar{V}(0)=H(0)
$$

Prova: ver Apêndice A .

Corolário 3.7. Para o modelo com n Ativos de Risco sem Cadeia de Markov, $H_{\theta(\tau)}(\tau)$, tal como definido na Equação (3.42) do Teorema 3.4, escreve-se como:

$$
H(\tau)=E_{\tau}^{P}\left\{m_{\mathcal{T}, \tau}^{P \rightarrow Q} \frac{H(\mathcal{T})}{\prod_{j=\tau}^{\mathcal{T}-1} a(j)}\right\}=E_{\tau}^{Q}\left\{\frac{H(\mathcal{T})}{\prod_{j=\tau}^{\mathcal{T}-1} a(j)}\right\},
$$

onde $m_{\mathcal{T}, \tau}^{P \rightarrow Q}$, dado por:

$$
m_{\mathcal{T}, \tau}^{P \rightarrow Q}=\prod_{j=\tau}^{\mathcal{T}-1} m_{j+1, j}^{P \rightarrow Q},
$$

corresponde à mudança de medida de probabilidade da medida $P$ para a medida $Q$.

Prova: ver Apêndice $A$. 


\subsection{Modelo com Um Ativo de Risco sem Cadeia de Markov}

Este modelo pode ser obtido a partir do modelo anterior simplesmente eliminandose a notação vetorial e adotando-se a notação escalar, já que há apenas um ativo de risco básico disponível no mercado.

Portanto, num modelo em que o mercado opere em apenas um modo, e havendo apenas um ativo de risco básico, os Teoremas 3.1, 3.2 e 3.4 podem ser rescritos como:

Corolário 3.8. Para cada $\tau=\mathcal{T}, \ldots, 0$, a estratégia ótima de controle do portfólio $\bar{u}(\tau)$ e a função objetivo ótima $\overline{\mathcal{J}}(V(\tau), \tau)$ são dadas por:

$$
\begin{gathered}
\bar{u}(\tau)=-\frac{E_{\tau}^{P}\left\{k(\tau+1) \Delta X(\tau+1)\left(\frac{V(\tau)}{S_{0}(\tau)}-\frac{H(\tau+1)}{S_{0}(\tau+1)}\right)\right\}}{E_{\tau}^{P}\left\{k(\tau+1)(\Delta X(\tau+1))^{2}\right\}}, \\
\mathcal{J}(V(\tau), \tau)=k(\tau)(V(\tau)-H(\tau))^{2}+\delta(\tau),
\end{gathered}
$$

onde, para $\tau=\mathcal{T}-1, \ldots, 0$ :

$$
\begin{gathered}
k(\tau)=a(\tau)^{2}\left[E_{\tau}^{P}\{k(\tau+1)\}-\frac{\left(E_{\tau}^{P}\{k(\tau+1) \Delta X(\tau+1)\}\right)^{2}}{E_{\tau}^{P}\left\{k(\tau+1)(\Delta X(\tau+1))^{2}\right\}}\right], \\
H(\tau)=E_{\tau}^{P}\left\{m_{\tau+1, \tau}^{P \rightarrow Q} \frac{H(\tau+1)}{a(\tau)}\right\} \\
m_{\tau+1, \tau}^{P \rightarrow Q}=\frac{k(\tau+1)-\frac{E_{\tau}^{P}\{k(\tau+1) \Delta X(\tau+1)\}}{E_{\tau}^{P}\left\{k(\tau+1)(\Delta X(\tau+1))^{2}\right\}} k(\tau+1) \Delta X(\tau+1)}{E_{\tau}^{P}\{k(\tau+1)\}-\frac{\left(E_{\tau}^{P}\{k(\tau+1) \Delta X(\tau+1)\}\right)^{2}}{E_{\tau}^{P}\left\{k(\tau+1)(\Delta X(\tau+1))^{2}\right\}}} \\
\delta(\tau)=E_{\tau}^{P}(\delta(\tau+1))+\quad \\
E_{\tau}^{P}\left\{k(\tau+1)(a(\tau) H(\tau)+S(\tau+1) \Delta X(\tau+1) u(\tau)-H(\tau+1))^{2}\right\} \geq 0 \\
u(\tau)=-\frac{E_{\tau}^{P}\left\{k(\tau+1) \Delta X(\tau+1)\left(\frac{H(\tau)}{S_{0}(\tau)}-\frac{H(\tau+1)}{S_{0}(\tau+1)}\right)\right\}}{E_{\tau}^{P}\left\{k(\tau+1)(\Delta X(\tau+1))^{2}\right\}}
\end{gathered}
$$

e as condições finais são:

$$
k(\mathcal{T})=1, \quad \delta(\mathcal{T})=0
$$

Prova: ver Apêndice $A$ 
Corolário 3.9. Seja $\overline{\mathcal{J}}(V(0), \theta(0), 0)$ tal como definido no Corolário 3.8. O valor de $V(0)$ que minimiza $\overline{\mathcal{J}}(V(0), \theta(0), 0)$, denominado por $\bar{V}(0)$, é dado por:

$$
\bar{V}(0)=H(0)
$$

Prova: ver Apêndice A

Corolário 3.10. Para o modelo com um Ativo de Risco sem Cadeia de Markov, $H_{\theta(\tau-1)}(\tau-1)$, tal como definido na Equação (3.42) do Teorema 3.4, escreve-se como:

$$
H(\tau)=E_{\tau}^{P}\left\{m_{\mathcal{T}, \tau}^{P \rightarrow Q} \frac{H(\mathcal{T})}{\prod_{j=\tau}^{\mathcal{T}-1} a(j)}\right\}=E_{\tau}^{Q}\left\{\frac{H(\mathcal{T})}{\prod_{j=\tau}^{\mathcal{T}-1} a(j)}\right\},
$$

onde $m_{\mathcal{T}, \tau}^{P \rightarrow Q}$, dado por:

$$
m_{\mathcal{T}, \tau}^{P \rightarrow Q}=\prod_{j=\tau}^{\mathcal{T}-1} m_{j+1, j}^{P \rightarrow Q},
$$

corresponde à mudança de medida de probabilidade da medida P para a medida $Q$.

Prova: ver Apêndice A 


\section{Capítulo 4}

\section{Modelo em Tempo Discreto, com Espaço de Estados Contínuo, com Um Ativo de Risco e sem Cadeia de Markov}

Neste item será desenvolvida a estrutura teórica que permite determinar o preço de um derivativo de característica européia e com payoff qualquer, bem como o controle ótimo da carteira que faz o hedging de mínima variância desse derivativo, num modelo de mercado em que o tempo é discreto e o espaço de estados é contínuo, atendendo assim às premissas que melhor refletem as condições de funcionamento do mercado financeiro real (conforme já descrito no Item 1.2). O modelo empregado para descrever a dinâmica dos retornos do ativo de risco corresponde a um processo de difusão com saltos (jump diffusion process).

No Item 4.1 são apresentados conceitos básicos preliminares sobre processos com saltos que serão empregados nos modelos desenvolvidos em itens a seguir. No Item 4.2 é estabelecida a equação de estado para $\Delta X(\cdot)$ num espaço de estados contínuo. Esta equação e outros resultados apresentados no apêndice desta tese são substituídos nas equações estabelecidas no Capítulo 3, Item 3.3, para produzir os resultados principais deste Capítulo 4, que são apresentados no Item 4.3. No Teorema 4.2 determinamos o fair hedging price do derivativo cujo payoff é $H(\mathcal{T})$. No Teorema 4.3 determinamos o controle ótimo da carteira que faz o hedging de mínima variância desse derivativo.

\subsection{Conceitos Preliminares}

Neste item apresentamos alguns conceitos preliminares acerca de processos com saltos.

Uma função $S:[0, T] \rightarrow \mathbb{R}^{d}$ é dita cadlag se ela é contínua à direita com 
limite à esquerda, i.e., se para cada $t \in[0, T]$, os limites:

$$
S(t-)=\lim _{u \rightarrow t, u<t} S(u) \quad S(t+)=\lim _{u \rightarrow t, u>t} S(u)
$$

existem e $S(t)=S(t+)$.

Sendo $t \in[0, T], S(t-)$ é conhecida como a versão contínua à esquerda de $S(t)$ no intervalo $[0, T]$.

Qualquer função contínua é cadlag, mas funções cadlag podem ter descontinuidades. Se $t$ é um ponto de descontinuidade, denotamos por:

$$
\Delta S(t)=S(t)-S(t-)
$$

o salto de $S$ no instante de tempo $t$, onde $t$ - corresponde ao instante de tempo imediatamente anterior a $t$.

Seja $Q(t)$ o seguinte processo composto de Poisson:

$$
Q(t)=\sum_{i=1}^{N^{P}(t)}\left(e^{Y_{i}^{P}}-1\right),
$$

onde $N^{P}(t)$ é um processo de Poisson de intensidade $\lambda^{P}$, e os $Y_{i}^{P}$ são variáveis aleatórias independentes e identicamente distribuídas com relação à medida de probabilidade $P$.

O incremento $\Delta Q(t)$ desse processo corresponde à variação de $Q(t)$ quando ocorre um salto, e é dado por:

$$
\Delta Q(t)=Q(t)-Q(t-)=\left(e^{Y_{i}^{P}}-1\right) .
$$

Usando a lei das expectativas iteradas, $E^{P}\{\cdot\}=E^{P}\left\{E^{P}\{\cdot\}\right\}$, o valor esperado desse processo é:

$$
\begin{gathered}
E^{P}\{Q(t)\}=E^{P}\left\{\sum_{i=1}^{N^{P}(t)}\left(e^{Y_{i}^{P}}-1\right)\right\}= \\
E^{P}\left\{E^{P}\left\{\sum_{i=1}^{N^{P}(t)}\left(e^{Y_{i}^{P}}-1\right) \mid N(t)=k\right\}\right\}= \\
E^{P}\left\{E^{P}\left\{\sum_{i=1}^{k}\left(e^{Y_{i}^{P}}-1\right)\right\}\right\}=E^{P}\left\{\sum_{i=1}^{k}\left(E^{P}\left\{e^{Y_{i}^{P}}\right\}-1\right)\right\} .
\end{gathered}
$$

Seja $\varphi_{Y_{i}}(u)$ a função geradora de momentos da VA $Y_{i}^{P}$, i.e.:

$$
\varphi_{Y_{i}^{P}}(u)=E^{P}\left\{e^{u Y_{i}^{P}}\right\} .
$$

Usando esta notação para continuar o desenvolvimento da esperança $E^{P}\{Q(t)\}$ 
acima (Equação (4.5)), obtemos:

$$
\begin{gathered}
E^{P}\{Q(t)\}=E^{P}\left\{\sum_{i=1}^{k}\left(\varphi_{Y_{i}^{P}}(1)-1\right)\right\}=E^{P}\left\{k\left(\varphi_{Y_{i}^{P}}(1)-1\right)\right\}= \\
\sum_{k=0}^{\infty} \frac{k\left(\varphi_{Y_{i}^{P}}(1)-1\right) e^{-\lambda t}\left(\lambda^{P} t\right)^{k}}{k !}=\left(\varphi_{Y_{i}^{P}}(1)-1\right) e^{-\lambda t} \sum_{k=0}^{\infty} \frac{k}{\left(\lambda^{P} t\right)^{k}}= \\
\left(\varphi_{Y_{i}^{P}}(1)-1\right) e^{-\lambda t} \sum_{k=1}^{\infty} \frac{k}{k !}=\left(\varphi_{Y_{i}^{P}}(1)-1\right) e^{-\lambda t} \lambda^{P} t \sum_{k=1}^{\infty} \frac{\left(\lambda^{P} t\right)^{k-1}}{(k-1) !}= \\
\left(\varphi_{Y_{i}^{P}}(1)-1\right) e^{-\lambda t} \lambda^{P} t \underbrace{\sum_{s=0}^{\infty} \frac{\left(\lambda^{P} t\right)^{s}}{s !}}_{e^{\lambda^{P} t}}=\left(\varphi_{Y_{i}^{P}}(1)-1\right) e^{-\lambda^{P} t} \lambda^{P} t e^{\lambda^{P} t}= \\
\left(\varphi_{Y_{i}^{P}}(1)-1\right) \lambda^{P} t .
\end{gathered}
$$

Portanto:

$$
E^{P}\{Q(t)\}=\left(\varphi_{Y_{i}^{P}}(1)-1\right) \lambda^{P} t .
$$

Consideremos que as variáveis aleatórias $Y_{i}^{P}$ possuam distribuição normal com $E\left\{Y_{i}\right\}=\beta_{Y}$ e $\sigma^{2}\left\{Y_{i}\right\}=\sigma_{Y}^{2}$, i.e., $Y_{i}^{P} \sim N\left(\beta_{Y}, \sigma_{Y}^{2}\right)$. Portanto:

$$
\varphi_{Y_{i}^{P}}(u)=E^{P}\left\{e^{u Y_{i}^{P}}\right\}=e^{u \beta_{Y}+\frac{1}{2} u^{2} \sigma_{Y}^{2}},
$$

e:

$$
\varphi_{Y_{i}^{P}}(1)=e^{\beta_{Y}+\frac{1}{2} \sigma_{Y}^{2}} .
$$

Assim, substituindo na equação para $E^{P}\{Q(t)\}$ (Equação (4.8)), obtemos:

$$
E^{P}\{Q(t)\}=\left(\varphi_{Y_{i}^{P}}(1)-1\right) \lambda^{P} t=\left(e^{\beta_{Y}+\frac{1}{2} \sigma_{Y}^{2}}-1\right) \lambda^{P} t .
$$

Seja $J(t)$ o seguinte processo puro de saltos:

$$
J(t)=\prod_{i=1}^{N^{P}(t)} e^{Y_{i}^{P}}=\exp \left\{\sum_{i=1}^{N^{P}(t)} Y_{i}^{P}\right\} .
$$

Sendo $t$ o instante de tempo em que ocorre o $i$-ésimo salto, e $t$ - o instante de tempo imediatamente anterior a esse instante, podemos escrever:

$$
J(t)=J(t-) e^{Y_{i}^{P}},
$$

e

$$
\begin{gathered}
\Delta J(t)=J(t)-J(t-)=J(t-) e^{Y_{i}^{P}}-J(t-)= \\
\quad J(t-)\left(e^{Y_{i}^{P}}-1\right)=J(t-) \Delta Q(t) .
\end{gathered}
$$


Novamente usando a lei das expectativas iteradas, o valor esperado do processo $(J(t))^{m}$ é:

$$
\begin{gathered}
E^{P}\left\{(J(t))^{m}\right\}=E^{P}\left\{\left(\prod_{i=1}^{N^{P}(t)} e^{Y_{i}^{P}}\right)^{m}\right\}= \\
E^{P}\left\{E^{P}\left\{\prod_{i=1}^{N^{P}(t)} e^{m Y_{i}^{P}} \mid N[t]=k\right\}\right\}=E^{P}\left\{\prod_{i=1}^{k}\left(E^{P}\left\{e^{m Y_{i}^{P}}\right\}\right)\right\}= \\
E^{P}\left\{\prod_{i=1}^{k} e^{m \beta_{Y}+\frac{1}{2} m^{2} \sigma_{Y}^{2}}\right\}=E^{P}\left\{\left(e^{m \beta_{Y}+\frac{1}{2} m^{2} \sigma_{Y}^{2}}\right)^{k}\right\}= \\
\sum_{k=0}^{\infty} \frac{\left(e^{m \beta_{Y}+\frac{1}{2} m^{2} \sigma_{Y}^{2}}\right)^{k}\left(e^{-\lambda \Delta t}\left(\lambda^{P} t\right)^{k}\right)}{k !}=e^{-\lambda \Delta t} \sum_{k=0}^{\infty} \frac{\left(\lambda^{P} t\left(e^{m \beta_{Y}+\frac{1}{2} m^{2} \sigma_{Y}^{2}}\right)\right)^{k}}{k !}= \\
e^{-\lambda^{P} t} e^{\lambda^{P} t e^{m \beta_{Y}+\frac{1}{2} m^{2} \sigma_{Y}^{2}}}=\exp \left[\lambda^{P} t\left(e^{m \beta_{Y}+\frac{1}{2} m^{2} \sigma_{Y}^{2}}-1\right)\right] .
\end{gathered}
$$

Aplicando esta expressão para $m=1$, temos:

$$
E^{P}\{J(t)\}=E^{P}\left\{\prod_{i=1}^{N^{P}(t)} e^{Y_{i}^{P}}\right\}=\exp \left[\lambda^{P} t\left(e^{\beta_{Y}+\frac{1}{2} \sigma_{Y}^{2}}-1\right)\right] .
$$

\subsection{Equações de Estado Para $\Delta X$ num Espaço de Estados Contínuo}

Neste item, estabelecemos as equações de estado para as variáveis $\Delta X(\cdot)$ e $S_{0}(\cdot)$, usadas nas equações do Corolário 3.8, para o modelo em que o espaço de estados é contínuo. Inicialmente, obtemos as equações de estado a tempo contínuo. Quando resolvidas, essas equações são então expressas a tempo discreto, gerando o modelo desejado: tempo discreto e espaço de estados contínuo.

Para relacionar instantes de tempo discretos aos seus instantes correspondentes contínuos, usamos a seguinte notação: os instantes de tempo contínuo $t_{0}$, $t_{1}=t_{0}+\Delta t_{0}, \ldots, t_{k+1}=t_{k}+\Delta t_{k}, \ldots, T=t_{\mathcal{T}}=t_{\mathcal{T}-1}+\Delta t_{\mathcal{T}-1}$ correspondem aos instantes de tempo discretos $\tau=0,1, \ldots, \mathcal{T}$.

No modelo a tempo contínuo, a equação diferencial que descreve a dinâmica do preço do ativo livre de risco é:

$$
d S_{0}(t)=S_{0}(t) r \mathrm{dt},
$$

onde $r$, taxa de juros livre de risco continuamente capitalizada, é suposta constante. Resolvendo esta simples equação diferencial, a solução para $S_{0}\left(t+\Delta t_{t}\right)$, a partir de uma condição inicial $\left(t, S_{0}(t)\right)$, é:

$$
S_{0}\left(t+\Delta t_{t}\right)=S_{0}(t) \exp \left(r \Delta t_{t}\right) .
$$

Deste ponto em diante, o preço $S\left(t_{f}\right)$ do ativo de risco num instante de tempo futuro $t_{f}>t, t$ sendo o instante de tempo atual, quando expresso em relação a uma medida de probabilidade $M$, é representado por $S^{M}\left(t_{f}\right)$. Quando 
o preço do ativo de risco for mensurável com relação ao conjunto de informações disponível no instante de tempo atual, a medida de probabilidade não é explicitada, já que não possui influência alguma.

Assim, para descrever a equação diferencial estocástica de $S(t)$ no modelo a tempo e espaço de estados contínuos, adotemos um processo de difusão com saltos. Então, expressemos pela seguinte equação diferencial estocástica a variação de $S(t)$ durante um intervalo de tempo $d t$ :

$$
\begin{gathered}
d S^{P}(t)=S^{P}(t+\mathrm{dt})-S(t)= \\
\underbrace{(\mu-\rho) S(t) d t}_{d R(t)}+\underbrace{\sigma S(t) d W^{P}(t)}_{d I(t)}+ \\
\underbrace{S(t-) d\left(Q^{P}(t)-\left(e^{\beta_{Y}+\frac{1}{2} \sigma_{Y}^{2}}-1\right) \lambda^{P} t\right)}_{d J(t)},
\end{gathered}
$$

onde $W^{P}(t)$ é um processo de Wiener em relação à medida de probabilidade $P$; $Q^{P}(t)$, definido na Equação (4.3), é um processo composto de Poisson em relação à medida de probabilidade $P$; e $\mu, \rho, \sigma, \beta_{Y}, \sigma_{Y}$ e $\lambda^{P}$ são parâmetros constantes ( $\mu$ corresponderia à taxa de retorno esperada da ação caso não houvesse pagamentos de dividendos; $\rho$ é a taxa de dividendos esperada da ação, pagos continuamente; como veremos a seguir, $\mu-\rho$ corresponde à taxa de retorno esperada da ação; $\sigma$ correponde ao coeficiente de difusão, i.e., à volatilidade, dos retornos do ativo; $\beta_{Y}, \sigma_{Y}$ e $\lambda^{P}$ foram definidos no item anterior).

O segundo termo do lado direito da Equação (4.19), $d I(t)$, corresponde a um processo de difusão representado por um movimento browniano geométrico. Esse termo representa variações estocásticas, porém contínuas, da variável de estado $S(t)$ durante um intervalo de tempo $d t$. O termo $d I(t)$ possui esperança nula:

$$
E_{t}^{P}\{d I(t)\}=E_{t}^{P}\left\{\sigma S(t) d W^{P}(t)\right\}=\sigma S(t) E_{t}^{P}\left\{d W^{P}(t)\right\}=0 .
$$

O terceiro termo do lado direito da Equação (4.19), $d J(t)$, corresponde a um processo de salto representado por um processo composto de Poisson. Esse termo representa possíveis variações estocásticas descontínuas da variável de estado $S(t)$ durante um intervalo de tempo $d t$. O termo $d J(t)$ possui esperança nula. De fato, usando a Equação (4.11):

$$
\begin{aligned}
E_{t}^{P}\{d J(t)\}= & E_{t}^{P}\left\{S(t-) d\left(Q(t)-\left(e^{\beta_{Y}+\frac{1}{2} \sigma_{Y}^{2}}-1\right) \lambda^{P} t\right)\right\}= \\
& S(t-) E_{t}^{P}\left\{d Q(t)-\left(e^{\beta_{Y}+\frac{1}{2} \sigma_{Y}^{2}}-1\right) \lambda^{P} \mathrm{dt}\right\}= \\
& S(t-)\left[d\left(E_{t}^{P}\left\{Q^{P}(t)\right\}\right)-\left(e^{\beta_{Y}+\frac{1}{2} \sigma_{Y}^{2}}-1\right) \lambda^{P} \mathrm{dt}\right]=0 .
\end{aligned}
$$

Por fim, o primeiro termo do lado direito da Equação (4.19), $d R(t)$, corresponde à variação esperada em $S(t)$ durante um intervalo de tempo $d t$. De fato:

$$
\begin{aligned}
E_{t}^{P}\left\{d S^{P}(t)\right\}= & E_{t}^{P}\{d R(t)+d I(t)+d J(t)\} \\
=E_{t}^{P}\{d R(t)\}+E_{t}^{P}\{d I(t)\}+ & E_{t}^{P}\{d J(t)\}= \\
& d R(t)=(\mu-\rho) S(t) d t .
\end{aligned}
$$


A Equação (4.19) pode ser rescrita como:

$$
\begin{aligned}
& d S^{P}(t)=(\mu-\rho) S(t) \mathrm{dt}+\sigma S(t) d W^{P}(t)+ \\
& \quad S(t-) d Q^{P}(t)-S(t-)\left(e^{\beta_{Y}+\frac{1}{2} \sigma_{Y}^{2}}-1\right) \lambda^{P} d t .
\end{aligned}
$$

Como $S(t-)$ é a versão contínua à esquerda de $S(t)$, estas funções apenas diferem em um conjunto de medida nula. Portanto, as integrais de $S(t) d t$ e de $S(t-) d t$ coincidem:

$$
\int_{0}^{u} S(t-) d t=\int_{0}^{u} S(t) d t,
$$

e a Equação (4.23) pode ser escrita como:

$$
\begin{aligned}
d S^{P}(t)=\left(\mu-\rho-\left(e^{\beta_{Y}+\frac{1}{2} \sigma_{Y}^{2}}-1\right) \lambda^{P}\right) & S(t) d t+ \\
& \sigma S(t) d W^{P}(t)+S(t-) d Q^{P}(t) .
\end{aligned}
$$

Para um instante de tempo $t+\Delta t_{t}$, a solução para $S^{P}\left(t+\Delta t_{t}\right)$, a partir de uma condição inicial $(t, S(t))$, é [32]:

$$
\begin{aligned}
S^{P}\left(t+\Delta t_{t}\right) & =S(t) \exp \left\{\sigma\left(W^{P}\left(t+\Delta t_{t}\right)-W^{P}(t)\right)+\right. \\
& \left.\left(\mu-\rho-\left(e^{\beta_{Y}+\frac{1}{2} \sigma_{Y}^{2}}-1\right) \lambda^{P}-\frac{1}{2} \sigma^{2}\right) \Delta t_{t}\right\} \prod_{i=1}^{N^{P}\left(\Delta t_{t}\right)} e^{Y_{i}^{P}} .
\end{aligned}
$$

Acrescentando a $S^{P}\left(t+\Delta t_{t}\right)$ os dividendos pagos pelo ativo de risco, no instante $t+\Delta t_{t}$, como remuneração durante os instantes de tempo $t$ e $t+\Delta t_{t}$, obtemos:

$$
\begin{gathered}
S^{P}\left(t+\Delta t_{t}\right)+\delta\left(t+\Delta t_{t}\right)=S^{P}\left(t+\Delta t_{t}\right) \exp \left(\rho \Delta t_{t}\right)= \\
S(t) \exp \left\{\sigma \Delta W^{P}\left(t+\Delta t_{t}\right)+\left(\mu-\left(e^{\beta_{Y}+\frac{1}{2} \sigma_{Y}^{2}}-1\right) \lambda^{P}-\frac{1}{2} \sigma^{2}\right) \Delta t_{t}\right\} \prod_{i=1}^{N^{P}\left(\Delta t_{t}\right)} e^{Y_{i}^{P}} .
\end{gathered}
$$

Rescrevendo as Equações (4.18) e (4.27) com a notação para tempo discreto, obtemos:

$$
S_{0}(\tau+1)=S_{0}(\tau) \exp \left(r \Delta t_{\tau}\right),
$$

$$
\begin{aligned}
& S^{P}(\tau+1)+\delta(\tau+1)= \\
& S(\tau) \exp \left\{\sigma \Delta W^{P}(\tau+1)+\left(\mu-\left(e^{\beta_{Y}+\frac{1}{2} \sigma_{Y}^{2}}-1\right) \lambda^{P}-\frac{1}{2} \sigma^{2}\right) \Delta t_{\tau}\right\} \prod_{i=1}^{N^{P}\left(\Delta t_{\tau}\right)} e^{Y_{i}^{P}} .
\end{aligned}
$$


Substituindo estas equações na Equação (3.10), obtemos para $\Delta X(\tau+1)$ :

$$
\begin{aligned}
& \Delta X(\tau+1)=\frac{S(\tau)}{S_{0}(\tau)}\left(\operatorname { e x p } \left\{\sigma \Delta W^{P}(\tau+1)+\right.\right. \\
& \left.\left.\quad\left(\mu-r-\left(e^{\beta_{Y}+\frac{1}{2} \sigma_{Y}^{2}}-1\right) \lambda^{P}-\frac{1}{2} \sigma^{2}\right) \Delta t_{\tau}\right\} \prod_{i=1}^{N^{P}\left(\Delta t_{\tau}\right)} e^{Y_{i}^{P}}-1\right) .
\end{aligned}
$$

Lembrando que:

- $\Delta W^{P}(\tau)$ é um processo de Wiener com $E_{\tau}^{P}\left\{\Delta W^{P}(\tau+1)\right\}=0$ e $E_{\tau}^{P}\left\{\left(\Delta W^{P}(\tau+\right.\right.$ 1) $\left.)^{2}\right\}=\Delta t_{\tau}$

- $N^{P}(\cdot)$ é um processo de Poisson de intensidade $\lambda^{P}$;

- Os $Y_{i}^{P}$ são variáveis aleatórias independentes e normalmente distribuídas com média $E_{\tau}^{P}\left\{Y_{i}^{P}\right\}=\beta_{Y}$ e desvio-padrão $\sigma_{\tau}^{P}\left\{Y_{i}^{P}\right\}=\sigma_{Y}$

- $S_{0}(\tau), S(\tau), \mu, \sigma$ e $r$ são constantes;

estabeleçamos o seguinte lema:

Lema 4.1. Seja $\Delta X(\tau+1)$ tal como definido na Equação (4.30). Considerando $\mu \neq r, \Delta X(\tau+1)$ pode ser expresso como:

$$
\begin{aligned}
& \Delta X(\tau+1)= \\
& \quad \frac{S(\tau)}{S_{0}(\tau)}\left(e^{(\mu-r) \Delta t_{\tau}}-1\right)\left(\alpha_{0}\left(\Delta t_{\tau}\right)+\alpha_{1}\left(\Delta t_{\tau}\right) Z_{1}\left(\Delta t_{\tau}\right) Z_{2}\left(\Delta t_{\tau}\right)\right),
\end{aligned}
$$

onde:

$$
\begin{gathered}
\alpha_{0}\left(\Delta t_{\tau}\right)=-\frac{1}{e^{(\mu-r) \Delta t_{\tau}-1}}, \\
\alpha_{1}\left(\Delta t_{\tau}\right)=\frac{e^{(\mu-r) \Delta t_{\tau}}}{e^{(\mu-r) \Delta t_{\tau}-1}}, \\
Z_{1}\left(\Delta t_{\tau}\right)=\exp \left\{\sigma \Delta W^{P}(\tau+1)-\frac{1}{2} \sigma^{2} \Delta t_{\tau}\right\} \\
Z_{2}\left(\Delta t_{\tau}\right)=e^{\left(\lambda^{P}-\lambda^{Q}\right) \Delta t_{\tau}} \prod_{i=1}^{N^{P}\left(\Delta t_{\tau}\right)} \frac{\lambda^{Q} f^{Q}\left(Y_{i}^{P}\right)}{\lambda^{P} f^{P}\left(Y_{i}^{P}\right)}, \\
\lambda^{Q}=\lambda^{P} e^{\frac{1}{2} \sigma_{Y}^{2}+\beta_{Y}}, \\
f_{i}^{Q}\left(Y_{i}^{P}\right)=\frac{1}{\sqrt{2 \pi} \sigma_{Y}} e^{-\frac{1}{2}\left(\frac{Y_{i}^{P}-\beta_{Y}}{\sigma_{Y}}\right)^{2}}, \\
\sqrt{2 \pi} \sigma_{Y} e^{-\frac{1}{2}\left(\frac{Y_{i}^{P}-\left(\sigma_{Y}^{2}+\beta_{Y}\right)}{\sigma_{Y}}\right)^{2}} .
\end{gathered}
$$

Prova: ver Apêndice A. 


\subsection{Resultados Principais}

Neste item são apresentados os resultados principais do Capítulo 4. No Teorema 4.2, apresentamos a expressão do fair hedging price do derivativo cujo payoff é $H(\mathcal{T})$. A seguir, no Teorema 4.3, determinamos o controle ótimo da carteira que faz o hedging de mínima variância desse derivativo.

Ambos os teoremas apresentados a seguir fazem uso da medida de probabilidade $Q_{l}$, definida no Lema A.8 por meio de sua derivada de Radon-Nikodým com relação à medida de probabilidade $P$. Essa medida, por sua vez, foi construída levando em conta que a dinâmica dos retornos do ativo de risco é descrita por um processo de difusão com saltos num espaço de estados contínuo.

Dado um derivativo com um payoff qualquer de característica européia, os resultados desses teoremas provêem a estrutura teórica a partir da qual se podem obter, caso haja tratabilidade matemática suficiente, expressões analíticas fechadas para o preço e para o controle ótimo da carteira que faz o hedging de mínima variância desse derivativo. Caso não haja a tratabilidade mencionada anteriormente, os resultados dos teoremas provêem, então, a estrutura adequada e direta para serem realizadas simulações de Monte Carlo produzindo numericamente o preço e o controle ótimo da carteira que faz o hedging de mínima variância do derivativo em questão.

Teorema 4.2. Sejam $H(\tau)$, tal como definido no Corolário 3.10, e $E_{\tau}^{Q_{l}}\{\cdot\}$, operador esperança condicional com relação à medida de probabilidade $Q_{l}$, cuja derivada de Radon-Nikodým com relação à medida de probabilidade $P, m_{\mathcal{T}}{ }_{{ }_{\tau}} Q_{l}$, encontra-se definida no Lema A.8. Então, $H(\tau)$ pode ser escrito como:

$$
\begin{aligned}
H(\tau)=e^{-r n \Delta t} \sum_{p=0}^{n}\left(a_{0}^{n-p} a_{1}^{p} \sum_{l=1}^{\left(\begin{array}{l}
n \\
p
\end{array}\right)} E_{\tau}^{P}\left\{m_{\mathcal{T}, \tau}^{P \rightarrow Q_{l}} H(\mathcal{T})\right\}\right)= \\
e^{-r n \Delta t} \sum_{p=0}^{n}\left(a_{0}^{n-p} a_{1}^{p} \sum_{l=1}^{\left(\begin{array}{l}
n \\
p
\end{array}\right)} E_{\tau}^{Q_{l}}\{H(\mathcal{T})\}\right),
\end{aligned}
$$

onde $n=\mathcal{T}-\tau, a_{0}$ e $a_{1}$ são dados por:

$$
\begin{aligned}
& a_{0}=\frac{e^{\Delta t\left(r+2 e^{\frac{1}{2} \sigma_{Y}^{2}+\beta_{Y}} \lambda^{P}-\lambda^{P}-\mu\right)}-e^{\Delta t\left(\sigma^{2}+e^{2\left(\sigma_{Y}^{2}+\beta_{Y}\right)} \lambda^{P}\right)}}{e^{\left(-1+2 e^{\frac{1}{2} \sigma_{Y}^{2}+\beta_{Y}}\right) \Delta t \lambda^{P}}-e^{\Delta t\left(\sigma^{2}+e^{2\left(\sigma_{Y}^{2}+\beta_{Y}\right)} \lambda^{P}\right)}},
\end{aligned}
$$

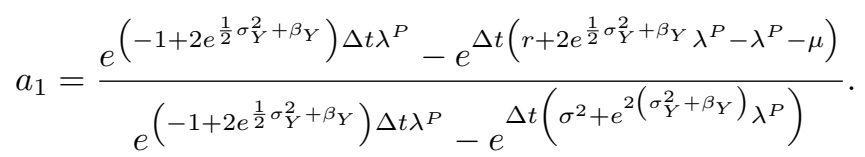

Prova: ver Apêndice A.

Teorema 4.3. Sejam $\Delta X(\tau+1)$ e $k(\tau+1)$ tais como definidos, respectivamente, nos Lemas 4.1 e A.5, $H(\tau+1)$ e $E_{\tau}^{Q_{l}}\{\cdot\}$ tais como definidos no Teorema 4.2, e $C_{p, l}^{n}$ e $C_{n-p, s}^{n}$ tais como definidos no Lema A.6. Então, a estratégia ótima $\bar{u}(\tau)$, dada pela Equação (3.56), pode ser escrita como: 


$$
\begin{gathered}
\bar{u}(\tau)=\left(e^{(\mu-r) \Delta t}-1\right) \frac{A-V(\tau)}{S(\tau) B}, \\
A=\sum_{p=0}^{n} e^{-r n \Delta t} a_{1}^{p} a_{0}^{n-p} \sum_{l=1}^{\left(\begin{array}{l}
n \\
p
\end{array}\right)}\left[\left(\frac{\alpha_{1}}{a_{1}}\right)^{\mathbf{I}_{C_{p, l}^{n}}(1)}\left(\frac{\alpha_{0}}{a_{0}}\right)^{\mathbf{I}_{C_{n-p, s}^{n}}(1)} E_{\tau}^{Q_{l}}\{H(\mathcal{T})\}\right], \\
B=\exp \left[\left(2 \mu-2 r+\sigma^{2}+\lambda^{P}\left(1-2 e^{\beta_{Y}+\frac{1}{2} \sigma_{Y}^{2}}+e^{2\left(\sigma_{Y}^{2}+\beta_{Y}\right)}\right)\right) \Delta t\right]- \\
2 \exp [(\mu-r) \Delta t]+1, \\
V(\tau)=e^{r \Delta t} V(\tau-1)+u(\tau-1)\left[S(\tau) e^{\rho \Delta t}-S(\tau-1) e^{r \Delta t}\right] .
\end{gathered}
$$

Prova: ver Apêndice A. 


\section{Capítulo 5}

\section{Aplicação}

Neste capítulo aplicamos os resultados apresentados no Capítulo 4 a uma opção de compra européia vanilla. Esta opção possui um payoff dado por:

$$
H(\mathcal{T})=\max \left(S^{Q_{l}}(\mathcal{T})-K, 0\right)=\left(S^{Q_{l}}(\mathcal{T})-K\right) \mathbf{I}_{\left\{S^{\left.Q_{l}(\mathcal{T})>K\right\}}\right.} .
$$

No Item 5.1 abordamos o caso em que os retornos do ativo de risco são descontínuos, pois são descritos por um processo de difusão com saltos. No Item 5.2, a dinâmica dos retornos do ativo de risco é simplificada para um processo browniano (processo de Wiener).

\subsection{Opção de Compra Européia Vanilla com Jumps}

A estrutura deste item está descrita a seguir.

No Lema 5.1 apresentamos o processo estocástico que descreve o preço do ativo de risco na data $\mathcal{T}, S^{Q_{l}}(\mathcal{T})$. O índice sobrescrito $Q_{l}$ apenas indica, como já explicado anteriormente, que o preço do ativo de risco está expresso em relação a uma medida de probabilidade $Q_{l}$.

No Teorema 5.2 apresentamos o fair hedging price da opção de compra européia quando o preço do ativo de risco é tal como descrito no Lema 5.1. No Teorema 5.3, apresentamos o controle ótimo da carteira que faz o hedging de mínima variância desse derivativo nas condições do Lema 5.1.

Os resultados dos Teoremas 5.2 e 5.3 correspondem a um paralelo do resultado apresentado por Merton [20] para o apreçamento de opções quando os retornos são descontínuos. A diferença entre esses resultados reside no fato de Merton ter considerado um modelo de mercado contínuo com relação ao tempo e ao espaço de estados, ao passo que aqui, mantendo o espaço de estados contínuo, consideramos um modelo discreto em relação ao tempo.

Lema 5.1. Seja $S^{P}(\cdot)$, preço do ativo de risco com relação à medida de probabilidade $P$, tal como definido na Equação (4.26). Então, $S^{Q_{l}}(\mathcal{T})$, o preço do ativo de risco com relação à medida de probabilidade $Q_{l}$ definida no Lema A.8, 
pode ser escrito como:

$$
\begin{aligned}
& S^{Q_{l}}(\mathcal{T})= \\
& S(\tau) \exp \left[\sum_{j=1}^{[\mathcal{T}-\tau} \sigma \Delta W^{Q_{l}}(\tau+j)+\sum_{i=1}^{N^{Q}((\mathcal{T}-\tau) \Delta t)}\left(Y_{i}^{Q}+\sigma_{Y}^{2}\right)+\right. \\
& \left.\quad\left(\mu-\rho-\left(e^{\beta_{Y}+\frac{1}{2} \sigma_{Y}^{2}}-1\right) \lambda^{P}-\frac{1}{2} \sigma^{2}\right)(\mathcal{T}-\tau) \Delta t+\sigma^{2} p \Delta t\right] .
\end{aligned}
$$

Prova: ver Apêndice A.

Teorema 5.2. Sejam $H(\tau)$ tal como definido no Teorema 4.2; $H(\mathcal{T})$, dado por:

$$
H(\mathcal{T})=\left(S^{Q_{l}}(\mathcal{T})-K\right) \mathbf{I}_{\left\{S^{Q_{l}}(\mathcal{T})>K\right\}}
$$

o payoff de uma European vanilla call option (i.e., opção de compra vanilla européia); e $S^{Q_{l}}(\mathcal{T})$ o preço do ativo de risco na data $\mathcal{T}$ tal como definido na Equação (5.2). Nessas condições, $H(\tau)$ pode ser escrito como:

$$
H(\tau)=e^{-r n \Delta t} \sum_{p=0}^{n}\left[\left(\begin{array}{l}
n \\
p
\end{array}\right) a_{0}^{n-p} a_{1}^{p} E_{\tau}^{Q_{l}}\{H(\mathcal{T})\}\right]
$$

onde:

$$
\begin{aligned}
& n=\mathcal{T}-\tau, \\
& a_{0}=\frac{e^{\Delta t\left(r+2 e^{\frac{1}{2} \sigma_{Y}^{2}+\beta_{Y}} \lambda^{P}-\lambda^{P}-\mu\right)}-e^{\Delta t\left(\sigma^{2}+e^{2\left(\sigma_{Y}^{2}+\beta_{Y}\right)} \lambda^{P}\right)}}{e^{\left(-1+2 e^{\frac{1}{2} \sigma_{Y}^{2}+\beta_{Y}}\right) \Delta t \lambda^{P}}-e^{\Delta t\left(\sigma^{2}+e^{2\left(\sigma_{Y}^{2}+\beta_{Y}\right)} \lambda^{P}\right)}},
\end{aligned}
$$

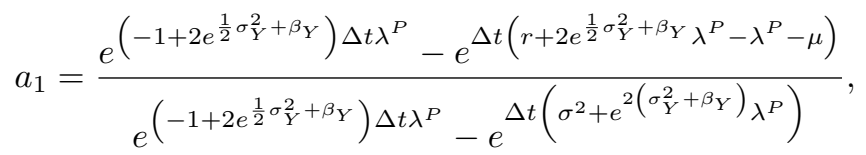

$$
\begin{aligned}
& E_{\tau}^{Q_{l}}\{H(\mathcal{T})\}=T_{1} G_{1}-K, \\
& T_{1}=S(\tau) e^{\left[(\mu-\rho)(\mathcal{T}-\tau)+\sigma^{2} p\right] \Delta t} \frac{\exp \left[\left(1-e^{\beta_{Y}+\frac{1}{2} \sigma_{Y}^{2}}\right) \lambda^{P}(\mathcal{T}-\tau) \Delta t\right]}{\exp \left[\left(1-e^{\beta_{Y}+\frac{3}{2} \sigma_{Y}^{2}}\right) \lambda^{Q}(\mathcal{T}-\tau) \Delta t\right]}, \\
& \lambda^{Q}=\lambda^{P} e^{\frac{1}{2} \sigma_{Y}^{2}+\beta_{Y}}, \\
& G_{1}=\sum_{\eta=0}^{\infty}\left(\frac{\phi\left(d_{R, p, \eta}\right)}{\eta !} \exp \left[-\lambda^{P} e^{2\left(\sigma_{Y}^{2}+\beta_{Y}\right)}(\mathcal{T}-\tau) \Delta t\right]\right. \\
& \left.\left(\lambda^{P} e^{2\left(\sigma_{Y}^{2}+\beta_{Y}\right)}(\mathcal{T}-\tau) \Delta t\right)^{\eta}\right)
\end{aligned}
$$




$$
\begin{aligned}
& G_{2}=\sum_{\eta=0}^{\infty}\left(\frac{\phi\left(d_{Q, p, \eta}\right)}{\eta !} \exp \left[-\lambda^{P} e^{\frac{1}{2} \sigma_{Y}^{2}+\beta_{Y}}(\mathcal{T}-\tau) \Delta t\right]\right. \\
& \left.\left(\lambda^{P} e^{\frac{1}{2} \sigma_{Y}^{2}+\beta_{Y}}(\mathcal{T}-\tau) \Delta t\right)^{\eta}\right) \\
& \phi(s)=\frac{1}{\sqrt{2 \pi}} \int_{-\infty}^{s} e^{-\frac{x^{2}}{2}} d x \\
& d_{Q, p, \eta}=\frac{A}{\left(\sigma^{2}(\mathcal{T}-\tau) \Delta t+\eta \sigma_{Y}^{2}\right)^{\frac{1}{2}}}, \\
& A=\ln \left(\frac{S(\tau)}{K}\right)+\sigma^{2} p \Delta t+\eta\left(\sigma_{Y}^{2}+\beta_{Y}\right)+ \\
& \left(\mu-\rho-\left(e^{\beta_{Y}+\frac{1}{2} \sigma_{Y}^{2}}-1\right) \lambda^{P}-\frac{1}{2} \sigma^{2}\right)(\mathcal{T}-\tau) \Delta t, \\
& d_{R, p, \eta}=\frac{B}{\left(\sigma^{2}(\mathcal{T}-\tau) \Delta t+\eta \sigma_{Y}^{2}\right)^{\frac{1}{2}}}, \\
& B=\ln \left(\frac{S(\tau)}{K}\right)+\sigma^{2} p \Delta t+\eta\left(2 \sigma_{Y}^{2}+\beta_{Y}\right)+ \\
& \left(\mu-\rho-\left(e^{\beta_{Y}+\frac{1}{2} \sigma_{Y}^{2}}-1\right) \lambda^{P}+\frac{1}{2} \sigma^{2}\right)(\mathcal{T}-\tau) \Delta t .
\end{aligned}
$$

Prova: ver Apêndice A.

Teorema 5.3. Sejam $\bar{u}(\tau)$ tal como definido no Teorema 4.3, $H(\mathcal{T})$, dado por:

$$
H(\mathcal{T})=\left(S^{Q_{l}}(\mathcal{T})-K\right) \mathbf{I}_{\left\{S^{Q_{l}}(\mathcal{T})>K\right\}}
$$

o payoff de uma European vanilla call option (i.e., opção de compra vanilla européia); e $S^{Q_{l}}(\mathcal{T})$ o preço do ativo de risco na data $\mathcal{T}$ tal como definido na Equação (5.2). Nessas condições, $\bar{u}(\tau)$ pode ser escrito como:

$$
\begin{gathered}
\bar{u}(\tau)=\left(e^{(\mu-r) \Delta t}-1\right) \frac{A-V(\tau)}{S(\tau) B} \\
A=\sum_{p=0}^{n} e^{-r n \Delta t} a_{1}^{p} a_{0}^{n-p}\left[\left(\begin{array}{c}
n-1 \\
p
\end{array}\right)\left(\frac{\alpha_{0}}{a_{0}}\right)+\left(\begin{array}{c}
n-1 \\
n-p
\end{array}\right)\left(\frac{\alpha_{1}}{a_{1}}\right)\right] E_{\tau}^{Q_{l}}\{H(\mathcal{T})\} \\
B=\exp \left[\left(2 \mu-2 r+\sigma^{2}+\lambda^{P}\left(1-2 e^{\beta_{Y}+\frac{1}{2} \sigma_{Y}^{2}}+e^{2\left(\sigma_{Y}^{2}+\beta_{Y}\right)}\right)\right) \Delta t\right]- \\
2 \exp [(\mu-r) \Delta t]+1
\end{gathered}
$$

onde: 


$$
\begin{aligned}
& n=\mathcal{T}-\tau, \\
& \alpha_{0}=-\frac{1}{e^{(\mu-r) \Delta t}-1}, \\
& \alpha_{1}=\frac{e^{(\mu-r) \Delta t}}{e^{(\mu-r) \Delta t}-1} \\
& a_{0}=\frac{e^{\Delta t\left(r+2 e^{\frac{1}{2} \sigma_{Y}^{2}+\beta_{Y}} \lambda^{P}-\lambda^{P}-\mu\right)}-e^{\Delta t\left(\sigma^{2}+e^{2\left(\sigma_{Y}^{2}+\beta_{Y}\right)} \lambda^{P}\right)}}{e^{\left(-1+2 e^{\frac{1}{2} \sigma_{Y}^{2}+\beta_{Y}}\right) \Delta t \lambda^{P}}-e^{\Delta t\left(\sigma^{2}+e^{2\left(\sigma_{Y}^{2}+\beta_{Y}\right)} \lambda^{P}\right)}}, \\
& a_{1}=\frac{e^{\left(-1+2 e^{\frac{1}{2} \sigma_{Y}^{2}+\beta_{Y}}\right) \Delta t \lambda^{P}}-e^{\Delta t\left(r+2 e^{\frac{1}{2} \sigma_{Y}^{2}+\beta_{Y}} \lambda^{P}-\lambda^{P}-\mu\right)}}{e^{\left(-1+2 e^{\frac{1}{2} \sigma_{Y}^{2}+\beta_{Y}}\right) \Delta t \lambda^{P}}-e^{\Delta t\left(\sigma^{2}+e^{2\left(\sigma_{Y}^{2}+\beta_{Y}\right)} \lambda^{P}\right)}}, \\
& E_{\tau}^{Q_{l}}\{H(\mathcal{T})\}=T_{1} G_{1}-K G_{2}, \\
& T_{1}=S(\tau) e^{\left[(\mu-\rho)(\mathcal{T}-\tau)+\sigma^{2} p\right] \Delta t} \frac{\exp \left[\left(1-e^{\beta_{Y}+\frac{1}{2} \sigma_{Y}^{2}}\right) \lambda^{P}(\mathcal{T}-\tau) \Delta t\right]}{\exp \left[\left(1-e^{\beta_{Y}+\frac{3}{2} \sigma_{Y}^{2}}\right) \lambda^{Q}(\mathcal{T}-\tau) \Delta t\right]}, \\
& \lambda^{Q}=\lambda^{P} e^{\frac{1}{2} \sigma_{Y}^{2}+\beta_{Y}}, \\
& G_{1}=\sum_{\eta=0}^{\infty}\left(\frac{\phi\left(d_{R, p, \eta}\right)}{\eta !} \exp \left[-\lambda^{P} e^{2\left(\sigma_{Y}^{2}+\beta_{Y}\right)}(\mathcal{T}-\tau) \Delta t\right]\right. \\
& \left.\left(\lambda^{P} e^{2\left(\sigma_{Y}^{2}+\beta_{Y}\right)}(\mathcal{T}-\tau) \Delta t\right)^{\eta}\right) \\
& G_{2}=\sum_{\eta=0}^{\infty}\left(\frac{\phi\left(d_{Q, p, \eta}\right)}{\eta !} \exp \left[-\lambda^{P} e^{\frac{1}{2} \sigma_{Y}^{2}+\beta_{Y}}(\mathcal{T}-\tau) \Delta t\right]\right. \\
& \left.\left(\lambda^{P} e^{\frac{1}{2} \sigma_{Y}^{2}+\beta_{Y}}(\mathcal{T}-\tau) \Delta t\right)^{\eta}\right) \\
& \phi(s)=\frac{1}{\sqrt{2 \pi}} \int_{-\infty}^{s} e^{-\frac{x^{2}}{2}} d x, \\
& d_{Q, p, \eta}=\frac{C}{\left(\sigma^{2}(\mathcal{T}-\tau) \Delta t+\eta \sigma_{Y}^{2}\right)^{\frac{1}{2}}},
\end{aligned}
$$




$$
\begin{gathered}
C=\ln \left(\frac{S(\tau)}{K}\right)+\sigma^{2} p \Delta t+\eta\left(\sigma_{Y}^{2}+\beta_{Y}\right)+ \\
\left(\mu-\rho-\left(e^{\beta_{Y}+\frac{1}{2} \sigma_{Y}^{2}}-1\right) \lambda^{P}-\frac{1}{2} \sigma^{2}\right)(\mathcal{T}-\tau) \Delta t \\
d_{R, p, \eta}=\frac{D}{\left(\sigma^{2}(\mathcal{T}-\tau) \Delta t+\eta \sigma_{Y}^{2}\right)^{\frac{1}{2}}}, \\
D=\ln \left(\frac{S(\tau)}{K}\right)+\sigma^{2} p \Delta t+\eta\left(2 \sigma_{Y}^{2}+\beta_{Y}\right)+ \\
\left(\mu-\rho-\left(e^{\beta_{Y}+\frac{1}{2} \sigma_{Y}^{2}}-1\right) \lambda^{P}+\frac{1}{2} \sigma^{2}\right)(\mathcal{T}-\tau) \Delta t \\
V(\tau)=e^{r \Delta t} V(\tau-1)+u(\tau-1)\left[S(\tau) e^{\rho \Delta t}-S(\tau-1) e^{r \Delta t}\right] .
\end{gathered}
$$

Prova: Ver apêndice A.

\subsection{Opção de Compra Européia Vanilla sem Jumps}

Neste item, particularizamos os resultados obtidos acima para o caso em que os retornos do ativo de risco são descritos por um processo de difusão sem saltos, i.e., por um processo browniano (ou de Wiener). A estrutura deste item está descrita a seguir.

No Teorema 5.4 apresentamos o fair hedging price da opção de compra européia vanilla quando o preço do ativo de risco segue um movimento browniano geométrico, e no Teorema 5.5 apresentamos o controle ótimo da carteira que faz o hedging de mínima variância desse derivativo nestas condições.

Os resultados dos Teoremas 5.4 e 5.5 correspondem a um paralelo do resultado apresentado por Black \& Scholes [3] para o apreçamento de opções quando os retornos seguem um movimento browniano. A diferença entre esses resultados reside no fato de Black \& Scholes terem considerado um modelo de mercado contínuo com relação ao tempo e ao espaço de estados, ao passo que aqui, mantendo o espaço de estados contínuo, consideramos um modelo discreto em relação ao tempo.

Teorema 5.4. Seja $H(\tau)$ tal como definido no Teorema 4.2. Seja ainda $H(\mathcal{T})$ dado por:

$$
H(\mathcal{T})=\left(S^{Q_{l}}(\mathcal{T})-K\right) \mathbf{I}_{\left\{S^{\left.Q_{l}(\mathcal{T})>K\right\}},\right.}
$$

correspondendo ao payoff de uma European vanilla call option (i.e., opção de compra vanilla européia). Nessas condições, $H(\tau)$ pode ser escrito como:

$$
H(\tau)=e^{-r n \Delta t} \sum_{p=0}^{n}\left[\left(\begin{array}{l}
n \\
p
\end{array}\right) a_{0}^{n-p} a_{1}^{p} E_{\tau}^{Q_{l}}\{H(\mathcal{T})\}\right],
$$


onde:

$$
\begin{gathered}
n=\mathcal{T}-\tau, \\
a_{0}=\frac{e^{(r-\mu) \Delta t}-e^{\sigma^{2} \Delta t}}{1-e^{\sigma^{2} \Delta t}}, \\
a_{1}=\frac{1-e^{(r-\mu) \Delta t}}{1-e^{\sigma^{2} \Delta t}}, \\
E_{\tau}^{Q_{l}}\{H(\mathcal{T})\}=S(\tau) e^{\left[(\mu-\rho)(\mathcal{T}-\tau)+\sigma^{2} p\right] \Delta t} \phi\left(d_{R, p}\right)-K \phi\left(d_{Q, p}\right), \\
\phi(s)=\frac{1}{\sqrt{2 \pi}} \int_{-\infty}^{s} e^{-\frac{x^{2}}{2}} d x, \\
d_{Q, p}=\frac{\ln \left(\frac{S(\tau)}{K}\right)+\left(\mu-\rho-\frac{1}{2} \sigma^{2}\right)(\mathcal{T}-\tau) \Delta t+\sigma^{2} p \Delta t}{\sigma \sqrt{(\mathcal{T}-\tau) \Delta t}}, \\
d_{R, p}=\frac{\ln \left(\frac{S(\tau)}{K}\right)+\left(\mu-\rho+\frac{1}{2} \sigma^{2}\right)(\mathcal{T}-\tau) \Delta t+\sigma^{2} p \Delta t}{\sigma \sqrt{(\mathcal{T}-\tau) \Delta t}} .
\end{gathered}
$$

Prova: ver Apêndice A.

Teorema 5.5. Seja $\bar{u}(\tau)$ tal como definido no Teorema 4.3. Seja ainda $H(\mathcal{T})$ dado por:

$$
H(\mathcal{T})=\left(S^{Q_{l}}(\mathcal{T})-K\right) \mathbf{I}_{\left\{S^{Q_{l}}(\mathcal{T})>K\right\}},
$$

correspondendo ao payoff de uma European vanilla call option (i.e., opção de compra vanilla européia). Nessas condições, $\bar{u}(\tau)$ pode ser escrito como:

$$
\begin{gathered}
\bar{u}(\tau)=\left(e^{(\mu-r) \Delta t}-1\right) \frac{A-V(\tau)}{S(\tau) B} \\
A=\sum_{p=0}^{n} e^{-r n \Delta t} a_{1}^{p} a_{0}^{n-p}\left[\left(\begin{array}{c}
n-1 \\
p
\end{array}\right)\left(\frac{\alpha_{0}}{a_{0}}\right)+\left(\begin{array}{c}
n-1 \\
n-p
\end{array}\right)\left(\frac{\alpha_{1}}{a_{1}}\right)\right] E_{\tau}^{Q_{l}}\{H(\mathcal{T})\} \\
B=e^{\left(2 \mu-2 r+\sigma^{2}\right) \Delta t}-2 e^{(\mu-r) \Delta t}+1
\end{gathered}
$$

onde:

$$
\begin{gathered}
n=\mathcal{T}-\tau, \\
\alpha_{0}=-\frac{1}{e^{(\mu-r) \Delta t}-1}, \\
\alpha_{1}=\frac{e^{(\mu-r) \Delta t}}{e^{(\mu-r) \Delta t}-1}, \\
a_{0}=\frac{e^{(r-\mu) \Delta t}-e^{\sigma^{2} \Delta t}}{1-e^{\sigma^{2} \Delta t}},
\end{gathered}
$$




$$
\begin{gathered}
a_{1}=\frac{1-e^{(r-\mu) \Delta t}}{1-e^{\sigma^{2} \Delta t}}, \\
E_{\tau}^{Q_{l}}\{H(\mathcal{T})\}=S(\tau) e^{\left[(\mu-\rho)(\mathcal{T}-\tau)+\sigma^{2} p\right] \Delta t} \phi\left(d_{R, p}\right)-K \phi\left(d_{Q, p}\right), \\
\phi(s)=\frac{1}{\sqrt{2 \pi}} \int_{-\infty}^{s} e^{-\frac{x^{2}}{2}} d x, \\
d_{Q, p}=\frac{\ln \left(\frac{S(\tau)}{K}\right)+\left(\mu-\rho-\frac{1}{2} \sigma^{2}\right)(\mathcal{T}-\tau) \Delta t+\sigma^{2} p \Delta t}{\sigma \sqrt{(\mathcal{T}-\tau) \Delta t}}, \\
d_{R, p}=\frac{\ln \left(\frac{S(\tau)}{K}\right)+\left(\mu-\rho+\frac{1}{2} \sigma^{2}\right)(\mathcal{T}-\tau) \Delta t+\sigma^{2} p \Delta t}{\sigma \sqrt{(\mathcal{T}-\tau) \Delta t}}, \\
V(\tau)=e^{r \Delta t} V(\tau-1)+u(\tau-1)\left[S(\tau) e^{\rho \Delta t}-S(\tau-1) e^{r \Delta t}\right] .
\end{gathered}
$$

Prova: ver Apêndice A. 


\section{Capítulo 6}

\section{Simulações Numéricas}

Neste item apresentamos simulações numéricas para o controle (i.e., hedging) de um portfólio composto apenas por uma opção de compra européia vanilla. A dinâmica do ativo base no caso estudado é dada por um movimento browniano geométrico.

Inicialmente, descrevemos detalhadamente a estrutura das simulações. Em seguida, apresentamos os resultados das simulações. Finalmente, comentamos os resultados obtidos nas simulações.

\subsection{Estrutura das Simulações}

Inicialmente, lembremo-nos de que o problema de hedging de mínima variância de um contrato derivativo não-realizável pode ser expresso como um problema de controle ótimo estocástico em tempo discreto e com variáveis de controle $V(0)$ e $\{u(\tau)\}_{\tau=0, \cdots, \mathcal{T}-1}$, dado por:

$$
\overline{\mathcal{J}}_{0, \mathcal{T}}(\theta(0), 0)=\underset{V(0),\{u(0), \ldots, u(\mathcal{T}-1)\} \in u_{0}}{\inf } E^{P}\left\{(V(\mathcal{T})-H(\mathcal{T}))^{2} \mid \mathcal{F}_{0}\right\}
$$

onde $V(\mathcal{T})$ é o valor do portfólio gerado pelas decisões de controle $\{u(\tau)\}_{\tau=0, \cdots, \mathcal{T}-1}$, e $H(\mathcal{T})$ é o valor terminal (i.e., o payoff) do derivativo não-realizável para o qual se pretende fazer o hedging.

As simulações numéricas realizadas nesta tese têm o objetivo de ilustrar o desempenho da estratégia ótima de controle obtida na solução do problema dado pela Equação $(6.1)$ quando $H(\mathcal{T})$ corresponde ao payoff de uma opção de compra européia vanilla, dado pela Equação (5.38). Como referência para avaliação dos resultados obtidos, comparamos os resultados gerados por esta estratégia ótima de controle com os obtidos com o uso da estratégia de hedging compatível com o modelo de Black \& Scholes, chamada de estratégia delta-neutra ou estratégia delta hedging $[35,16]$. A estratégia delta-neutra foi escolhida porque, além de extremamente simples, é a estratégia amplamente usada pelos profissionais do mercado financeiro para o hedging da opção em questão.

A estrutura das simulações realizadas é a seguinte. Inicialmente, identificamos o número $n$ de rebalanceamentos, i.e., de ações de controle, que haverá no portfólio de hedging, desde o instante de tempo atual (i.e., $\tau=0$ na notação de 
tempo discreto, e $t_{0}$ na notação de tempo contínuo), até o instante de maturidade da opção (i.e., $\mathcal{T}$ na notação de tempo discreto, e $t_{\mathcal{T}}$ na notação de tempo contínuo). O número de ações de controle é dado por $n=\mathcal{T}-\tau=\mathcal{T}$. Conforme definições apresentadas no Item $4.2, n$ pode ser calculado como:

$$
n=\mathcal{T}-\tau=\frac{t_{\mathcal{T}}-t_{0}}{\Delta t}=\frac{t_{\mathcal{T}}}{\Delta t},
$$

onde $\Delta t$ é o intervalo de tempo entre duas ações de controle consecutivas (i.e., entre dois rebalanceamentos consecutivos do portfólio).

De posse de $n$ e do valor do ativo base no instante de tempo atual $\tau=0$, geramos uma seqüência de valores para o ativo base nos $n$ instantes de tempo em que há rebalanceamentos. Isto é feito usando-se sucessivamente a Equação (4.26), simplificada para o caso em que não há saltos:

$$
S_{i}(j+1)=S_{i}(j) \exp \left[\left(\mu-\rho-\frac{1}{2} \sigma^{2}\right) \Delta t+\sigma\left(W_{j+1}-W_{j}\right)\right],
$$

onde $j=0, \ldots, \mathcal{T}-1$ e o índice $i, i=1, \ldots, N_{\text {sim }}$, indica apenas que se trata da $i$-ésima simulação (ou da $i$-ésima trajetória) num conjunto de $N_{\text {sim }}$ simulações. Esses $n=\mathcal{T}$ valores de $S_{i}(\cdot)$ gerados representam uma possível trajetória realizada pelo preço ativo de risco desde o instante de tempo atual $\tau=0$ até o instante de maturidade $\mathcal{T}$ do derivativo. A partir dessa trajetória de preços simulada, calculamos $H_{i}(\mathcal{T})$ (Equação (5.38)).

Chamemos de $V_{i}^{\mathrm{PD}}(\mathcal{T})$ o valor do portfólio que é gerado pelas decisões de controle $\{u(\tau)\}_{\tau=0, \cdots, \mathcal{T}-1}$ obtidas a partir da solução do problema dado pela Equação (6.1) e aplicadas à $i$-ésima trajetória de preços simulada. Chamemos de $V_{i}^{\mathrm{BS}}(\mathcal{T})$ o valor do portfólio gerado por decisões de controle $\{u(\tau)\}_{\tau=0, \cdots, \mathcal{T}-1}$ obtidas a partir do modelo de Black \& Scholes (estratégia delta-neutra) e aplicadas à $i$-ésima trajetória de preços simulada.

Assim, de posse da $i$-ésima trajetória de preços simulada, usamos as equações estabelecidas nos Teoremas 5.4 e 5.5 para encontrar $V_{i}^{\mathrm{PD}}(\mathcal{T})$. De forma análoga, usamos a equação de controle para a estratégia delta-neutra $[35,16]$ e a Equação (5.60) para encontrar $V_{i}^{\mathrm{BS}}(\mathcal{T})$. Então, para esta $i$-ésima trajetória de preços simulada, calculamos o erro quadrático (chamado de $\mathrm{eq}_{i}^{\mathrm{PD}}$ ) de $V_{i}^{\mathrm{PD}}(\mathcal{T})$ em relação a $H_{i}(\mathcal{T})$, dado por:

$$
\mathrm{eq}_{i}^{\mathrm{PD}}=\left(V_{i}^{\mathrm{PD}}(\mathcal{T})-H_{i}(\mathcal{T})\right)^{2},
$$

e, analogamente, o erro quadrático (chamado de $\mathrm{eq}_{i}^{\mathrm{BS}}$ ) de $V_{i}^{\mathrm{BS}}(\mathcal{T})$ em relação a $H_{i}(\mathcal{T})$, dado por:

$$
\mathrm{eq}_{i}^{\mathrm{BS}}=\left(V_{i}^{\mathrm{BS}}(\mathcal{T})-H_{i}(\mathcal{T})\right)^{2}
$$

Finalmente, repetimos esse procedimento $N_{\text {sim }}$ vezes, obtendo $N_{\text {sim }}$ valores para $\mathrm{eq}_{i}^{\mathrm{PD}}$ e para $\mathrm{eq}_{i}^{\mathrm{PD}}$. A lei forte dos grandes números $[15,22]$ assegura então que, com probabilidade 1:

$$
\begin{aligned}
E^{P}\left\{\left(V^{\mathrm{PD}}(\mathcal{T})-H(\mathcal{T})\right)^{2} \mid \mathcal{F}_{0}\right\} & = \\
\lim _{N_{\mathrm{sim}} \rightarrow \infty} & {\left[\frac{1}{N_{\text {sim }}}\left(\sum_{i=1}^{N_{\mathrm{sim}}}\left(V_{i}^{\mathrm{PD}}(\mathcal{T})-H_{i}(\mathcal{T})\right)^{2}\right)\right] }
\end{aligned}
$$


e:

$$
\begin{aligned}
E^{P}\left\{\left(V^{\mathrm{BS}}(\mathcal{T})-H(\mathcal{T})\right)^{2} \mid \mathcal{F}_{0}\right\}= & \\
\lim _{N_{\mathrm{sim}} \rightarrow \infty} & {\left[\frac{1}{N_{\mathrm{sim}}}\left(\sum_{i=1}^{N_{\text {sim }}}\left(V_{i}^{\mathrm{BS}}(\mathcal{T})-H_{i}(\mathcal{T})\right)^{2}\right)\right], }
\end{aligned}
$$

ou seja, a esperança do erro quadrático de hedging pode ser aproximada pela média aritmética dos erros quadráticos de hedging (i.e., pelo erro quadrático médio de hedging) obtidos nas $N_{\text {sim }}$ simulações.

Definamos como REQM(PD) a raiz quadrada do erro quadrático médio de hedging obtido nas simulações em que foi usada a estratégia de hedging derivada pela técnica de programação dinâmica, e como $\operatorname{REQM}(\mathrm{BS})$ a raiz quadrada do erro quadrático médio de hedging obtido nas simulações em que foi usada a estratégia de hedging derivada do modelo de Black \& Scholes (delta-neutra). Assim:

$$
\begin{aligned}
& \operatorname{REQM}(\mathrm{PD})=\sqrt{\frac{1}{N_{\text {sim }}} \sum_{i=1}^{N_{\text {sim }}} \mathrm{eq}_{i}^{\mathrm{PD}},} \\
& \operatorname{REQM}(\mathrm{BS})=\sqrt{\frac{1}{N_{\text {sim }}} \sum_{i=1}^{N_{\text {sim }}} \mathrm{eq}_{i}^{\mathrm{BS}} .}
\end{aligned}
$$

Esses valores provêem uma estimativa do erro esperado de hedging de uma opção de compra vanilla européia quando usamos a estratégia de hedging obtida com a técnica de controle ótimo estocástico (programação dinâmica estocástica), e quando usamos a estratégia de hedging derivada do modelo de Black \& Scholes (delta-neutra).

Em cada um dos gráficos do item seguinte, representamos os resultados obtidos para $H_{i}(\mathcal{T}), V_{i}^{\mathrm{PD}}(\mathcal{T})$ e $V_{i}^{\mathrm{BS}}(\mathcal{T})$ em $N_{\text {sim }}=1.500$ simulações de trajetórias de preços do ativo base. Cada um desses gráficos corresponde a uma determinada opção de compra vanilla européia (refletida pelo preço de exercício $K$ da opção), numa determinada condição de mercado (refletida no retorno esperado do ativo e na volatilidade desse retorno), e com um determinado número de rebalanceamentos (i.e., ações de controle). A Tabela (6.1) define as 36 diferentes situações consideradas:

Com relação ao preço de exercício das opções, consideramos os três tipos de casos possíveis: opções dentro do dinheiro, no dinheiro e fora do dinheiro. Apenas lembrando, uma opção de compra está dentro do dinheiro quando $S>$ $K$, no dinheiro quando $S=K$ e fora do dinheiro quando $S<K$. Entretanto, aqui, essa classificação foi considerada com relação ao preço forward $F_{0}$ do ativo base $^{1}$, dado por:

$$
\begin{aligned}
F_{0} & =E^{P}\left\{S(\mathcal{T}) \mid \mathcal{F}_{0}\right\}= \\
& E^{P}\left\{S(0) \exp \left[\left(\mu-\rho-\frac{1}{2} \sigma^{2}\right) \mathcal{T}+\sigma\left(W_{\mathcal{T}}-W_{0}\right)\right] \mid \mathcal{F}_{0}\right\}=S(0) e^{(\mu-\rho) \mathcal{T}} .
\end{aligned}
$$

\footnotetext{
${ }^{1}$ Assim, a classificação dentro , no e fora do dinheiro passam a ser dadas, respectivamente, por $F_{0}>K, F_{0}=K$ e $F_{0}<K$.
} 
Tabela 6.1: Parâmetros usados nas simulações

\begin{tabular}{lc} 
Variável & Valores utilizados \\
\hline Prazo de vencimento da opção & $T=1$ ano \\
Taxa de juros livre de risco & $r=15 \%$ a.a. \\
Preço inicial do ativo base & $S(0)=100$ \\
Taxa de dividendos do ativo base & $\rho=0$ \\
Taxa de retorno esperada do ativo & $\mu=20 \%$ a.a., $10 \% a . a .,-5 \% a . a$. \\
Volatilidade do ativo & $\sigma=30 \%$ a.a., $45 \%$ a.a. \\
Preço de exercício da opção (K) & Dentro/no/fora do dinheiro forward \\
Número de rebalanceamentos & $n=6,12$
\end{tabular}

Além disso, os gráficos mostram outras informações. Duas delas, REQM(PD) e REQM(BS), são definidas nas Equações (6.8) e (6.9), respectivamente. Para dar maior significado a estas variáveis, elas estão expressas em valor presente e em relação ao valor de $H(0)=H_{0}$. Assim, um REQM(PD) de, por exemplo, $17 \%$, indica que a raiz quadrada do erro quadrático esperado de hedging, expresso em valores monetários para a data atual (i.e., em valor presente), corresponde a $17 \%$ do valor $H(0)$ da opção. A variável "REQM relat." nos gráficos corresponde simplesmente a:

$$
\mathrm{REQM} \text { relat } .=\frac{\operatorname{REQM}(\mathrm{PD})}{\mathrm{REQM}(\mathrm{BS})}-1 .
$$

Por fim, a variável "\% $\mathrm{PD}<\mathrm{BS} "$, também mostrada nos gráficos, indica o percentual de vezes em que a estratégia de hedging obtida por programação dinâmica estocástica produziu melhor resultado, i.e., apresentou menor erro quadrático, que a estratégia de hedging de Black \& Scholes.

\subsection{Resultados das Simulações}

Neste item apresentamos os gráficos, contidos nas Figuras (6.1) a (6.12), com os resultados das simulações, tal como descrito no item anterior. 


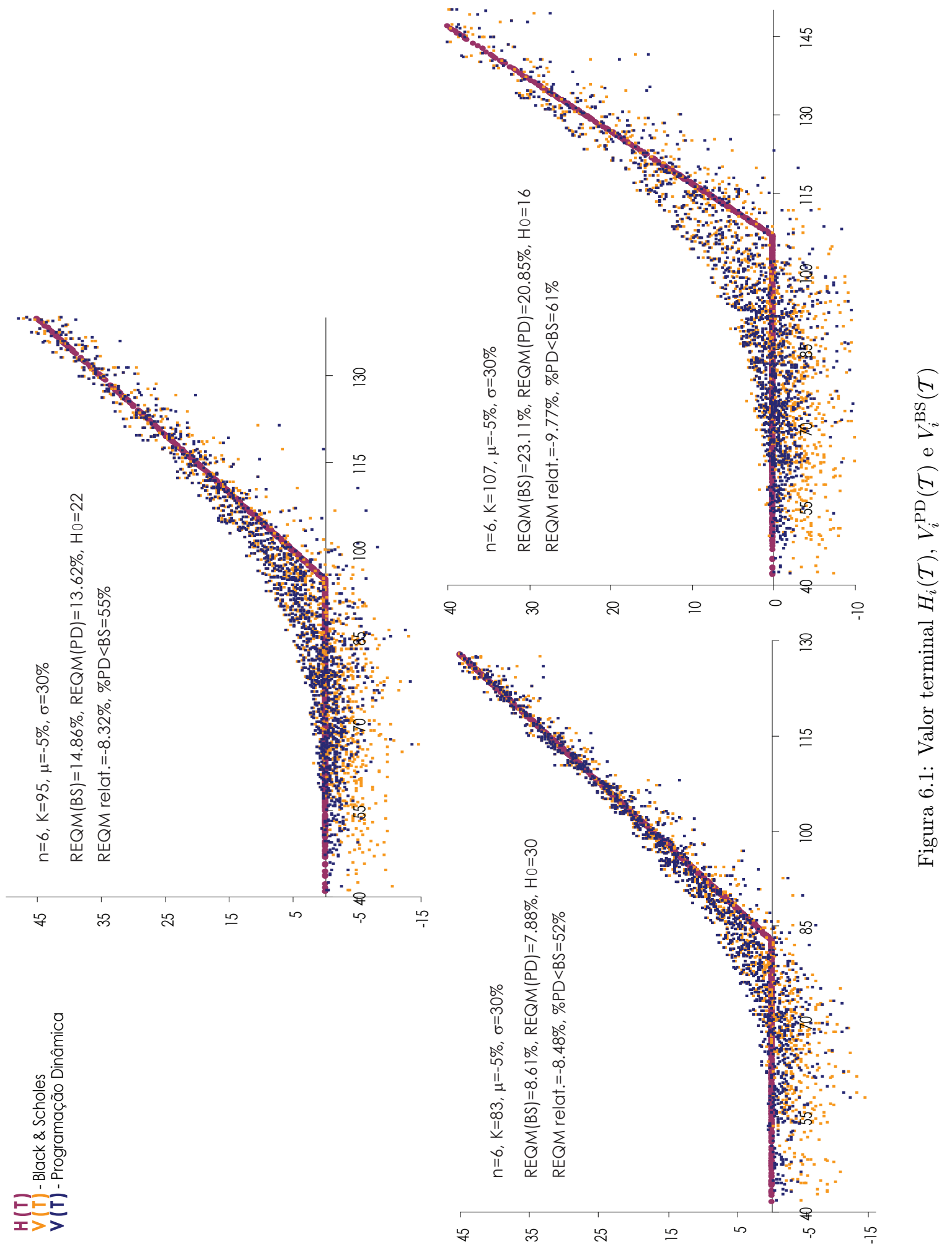



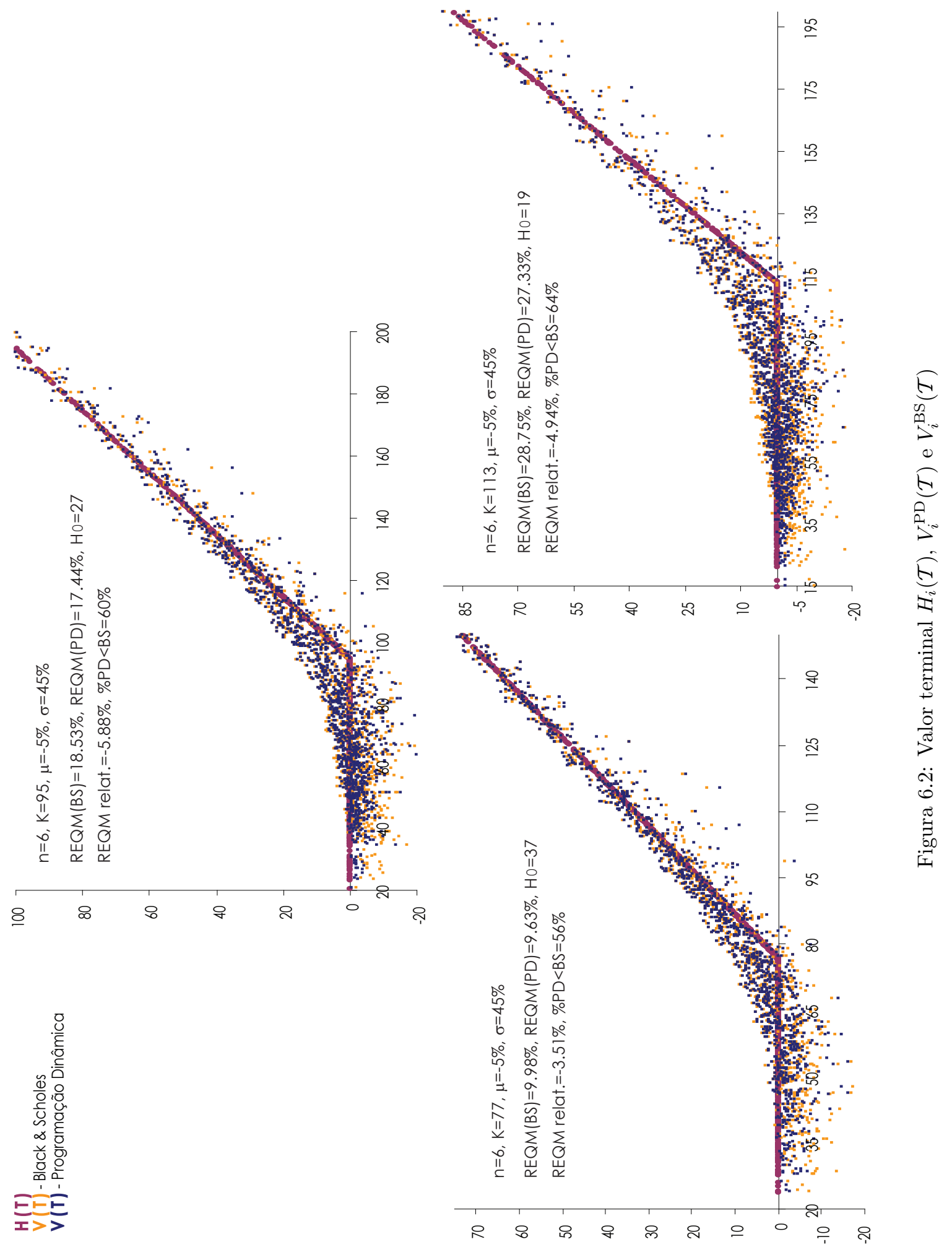


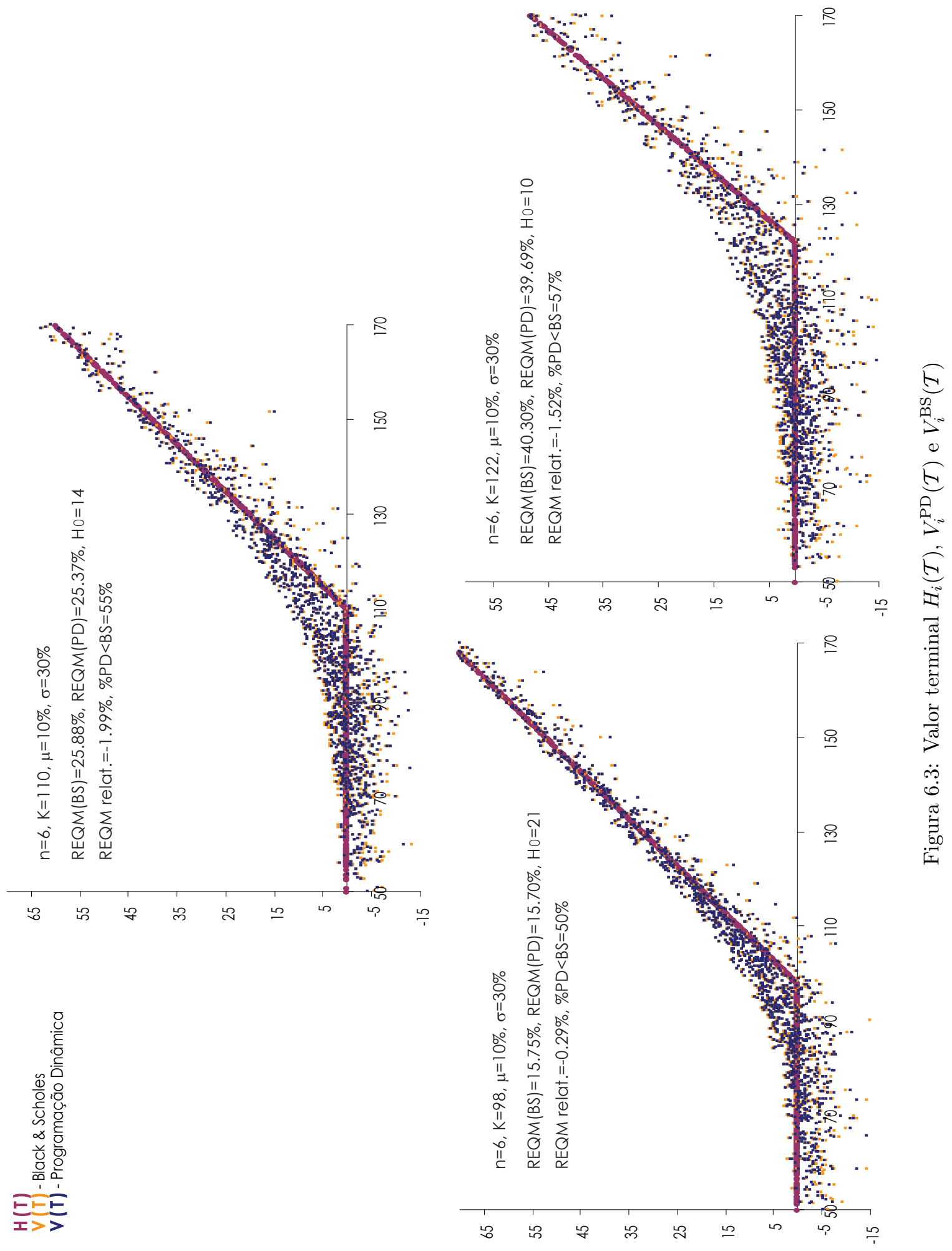

55 


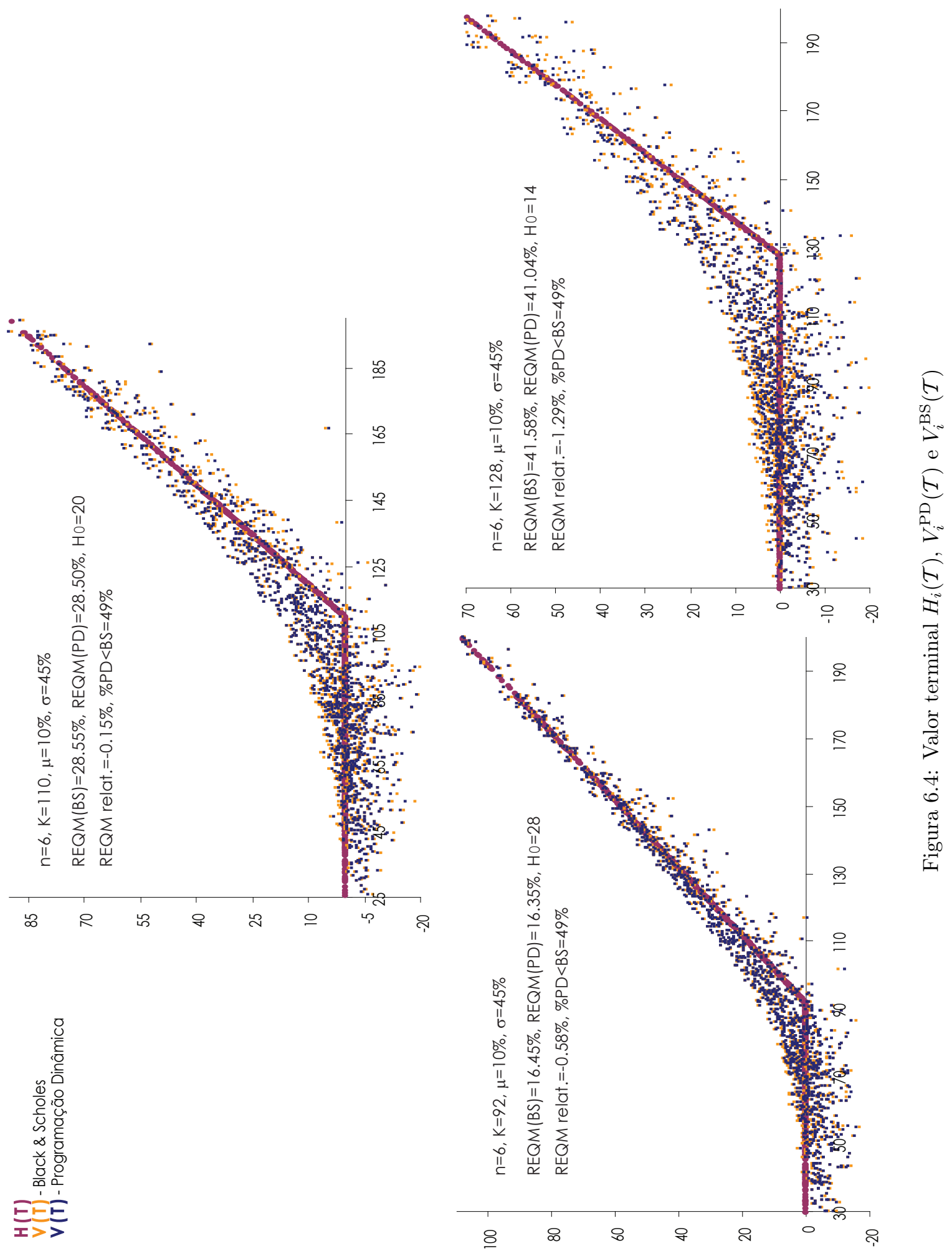

56 

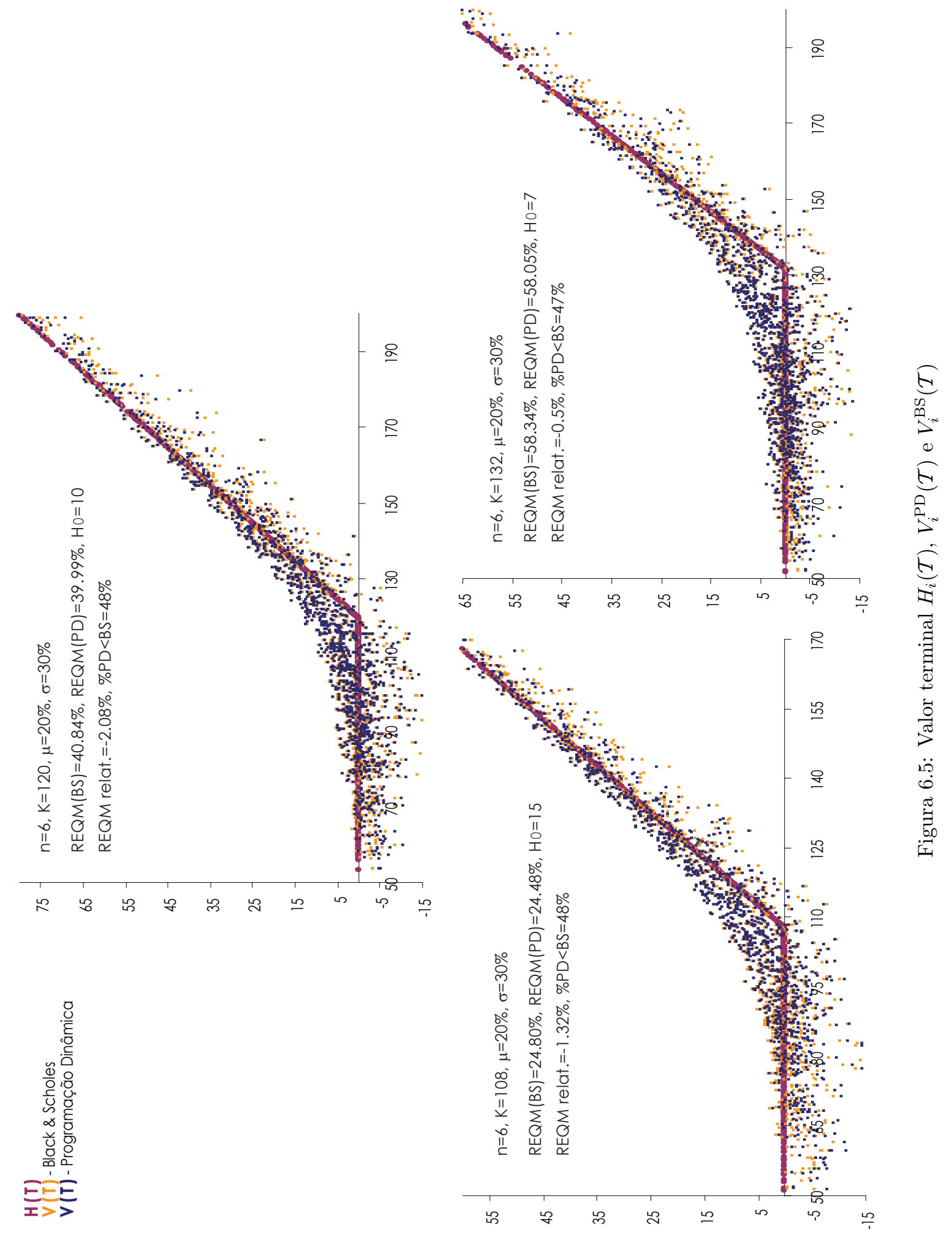

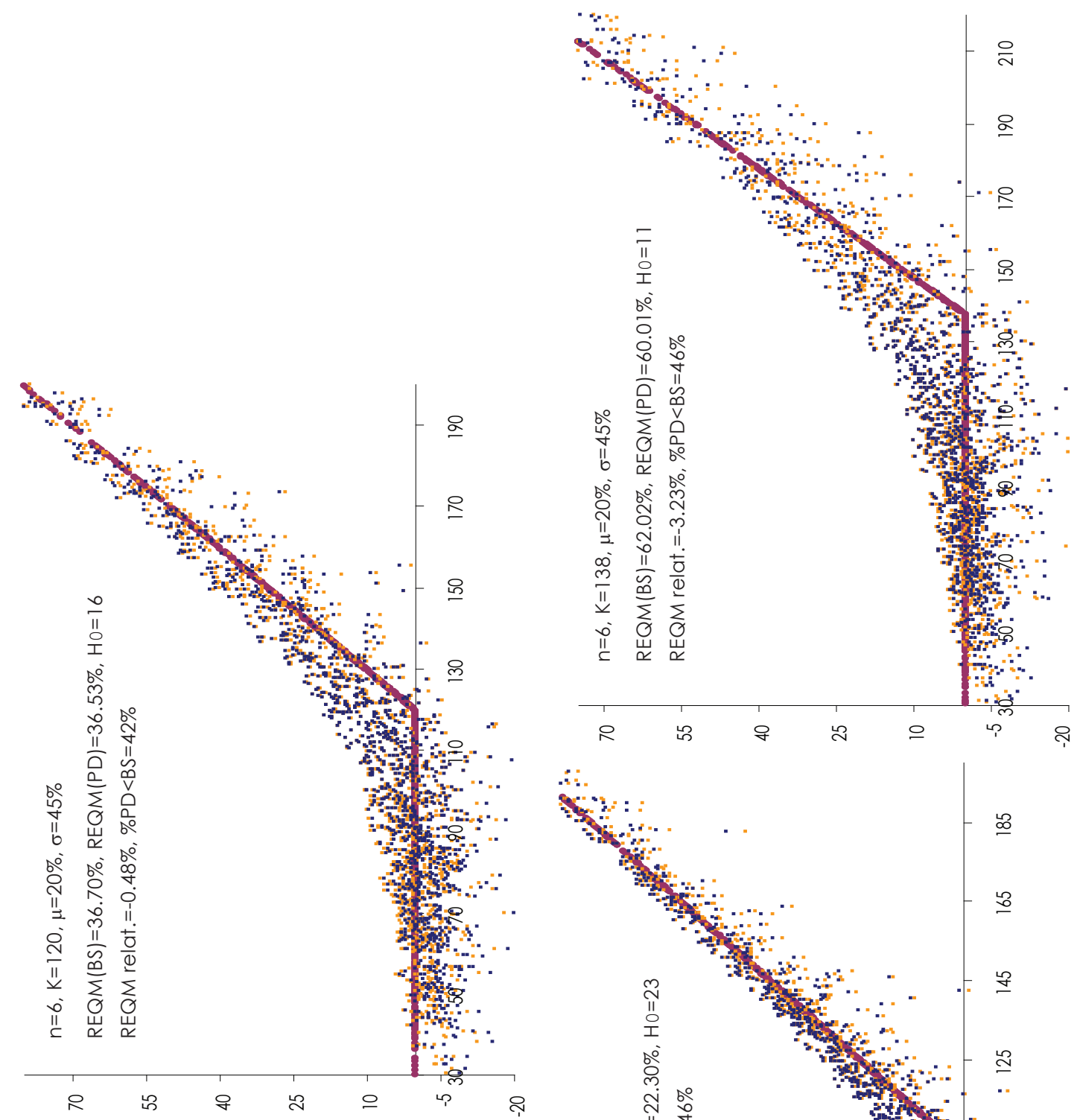

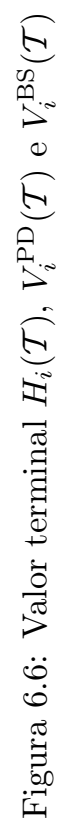
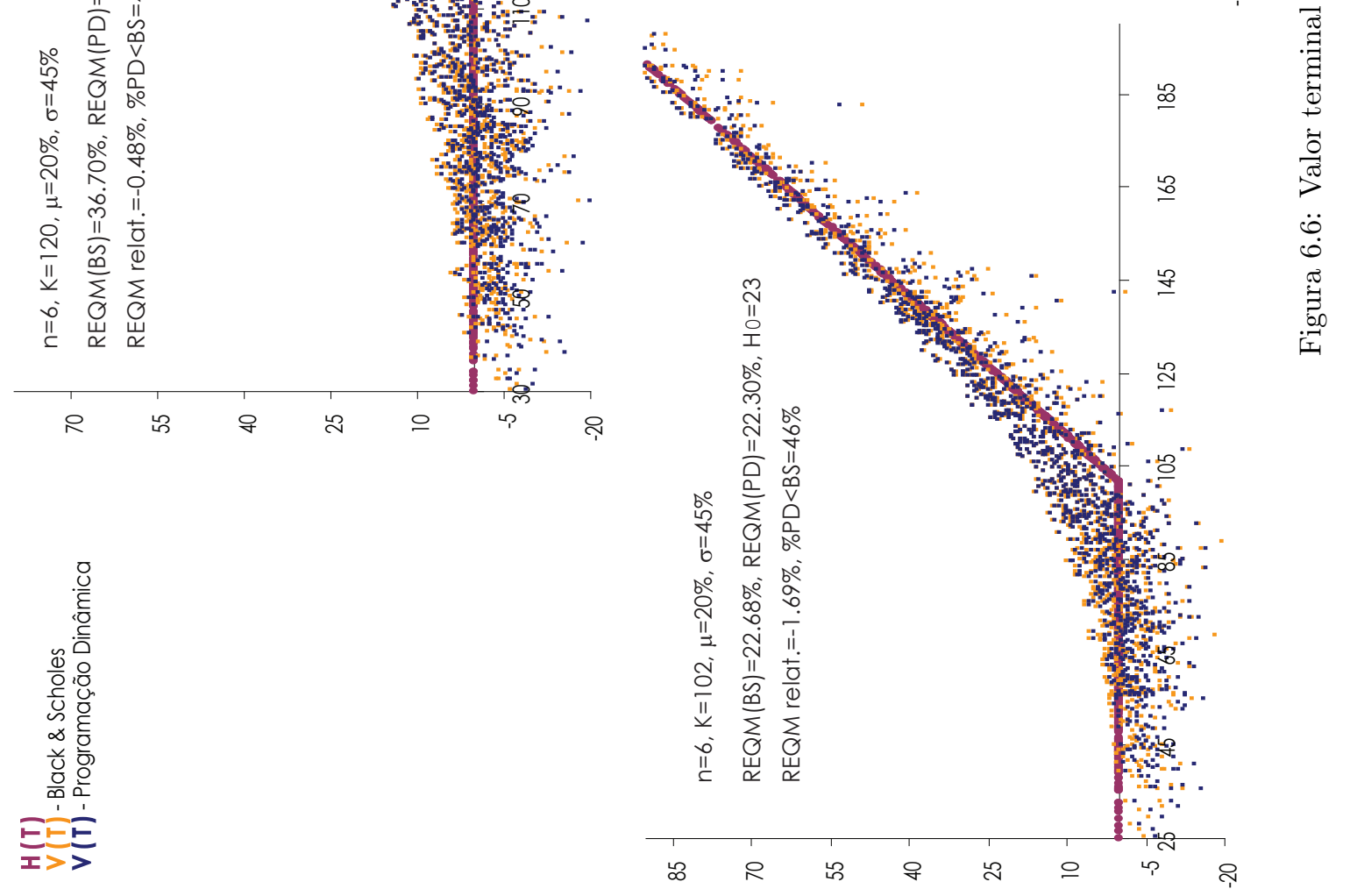


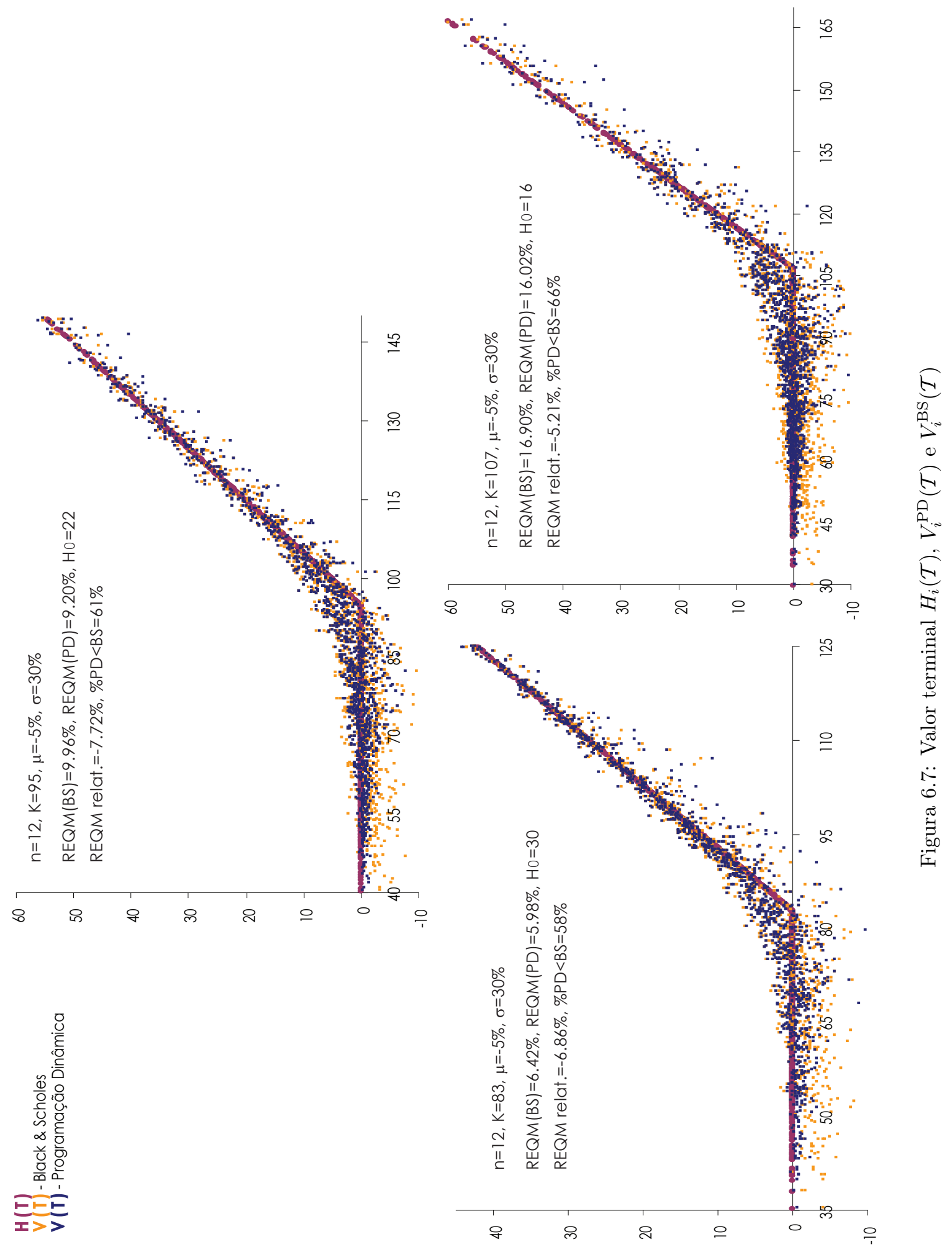




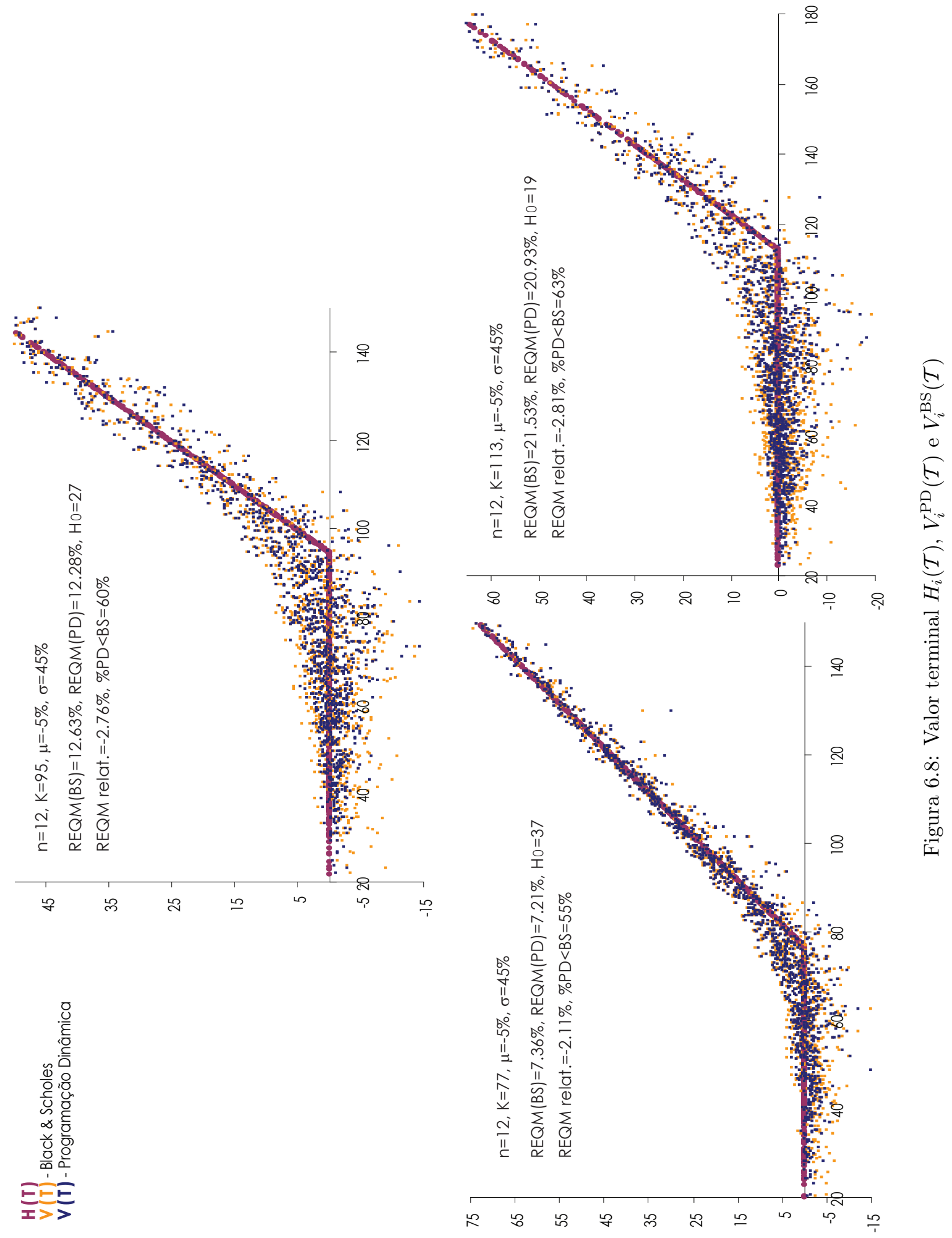

60 


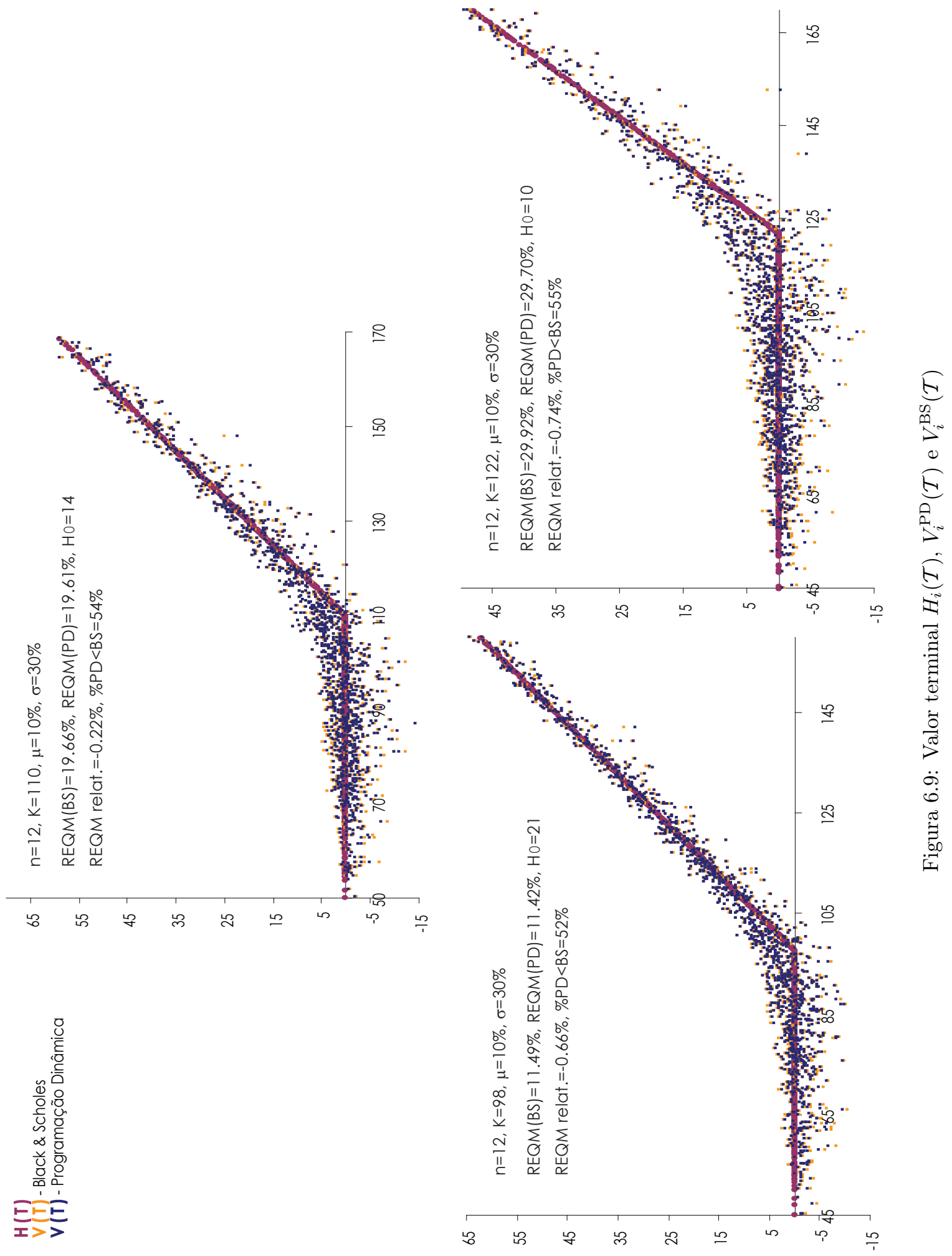

61 


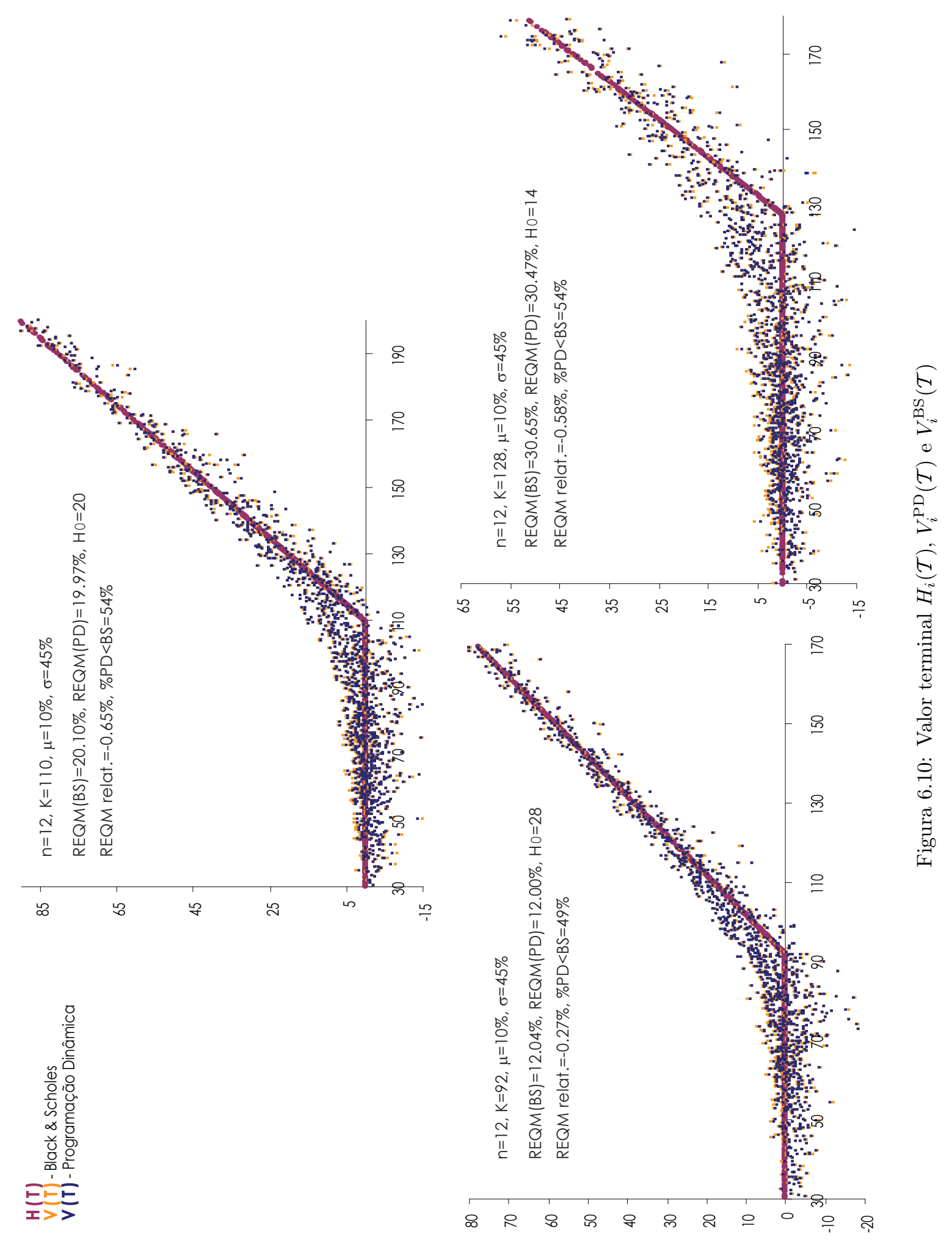




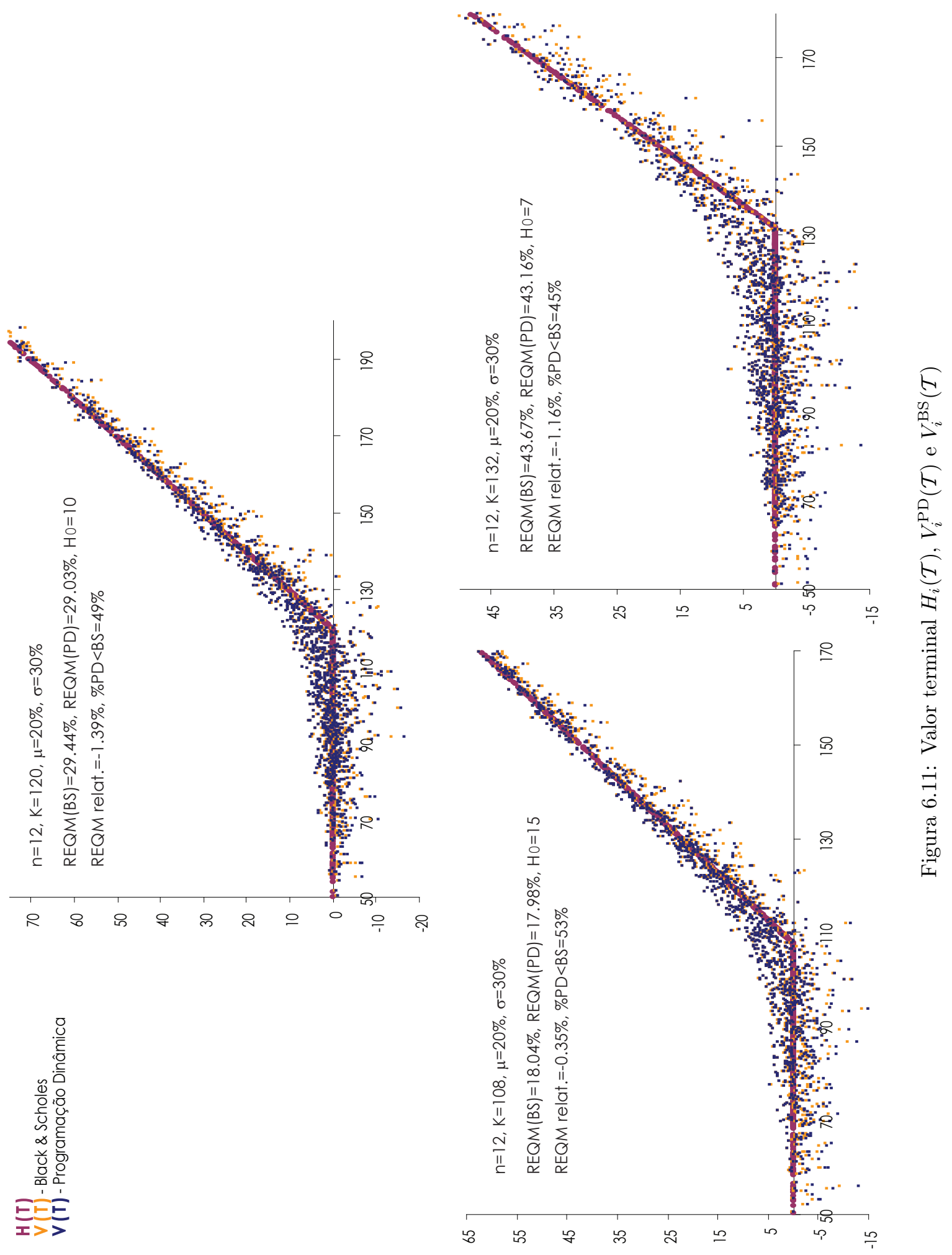

63 

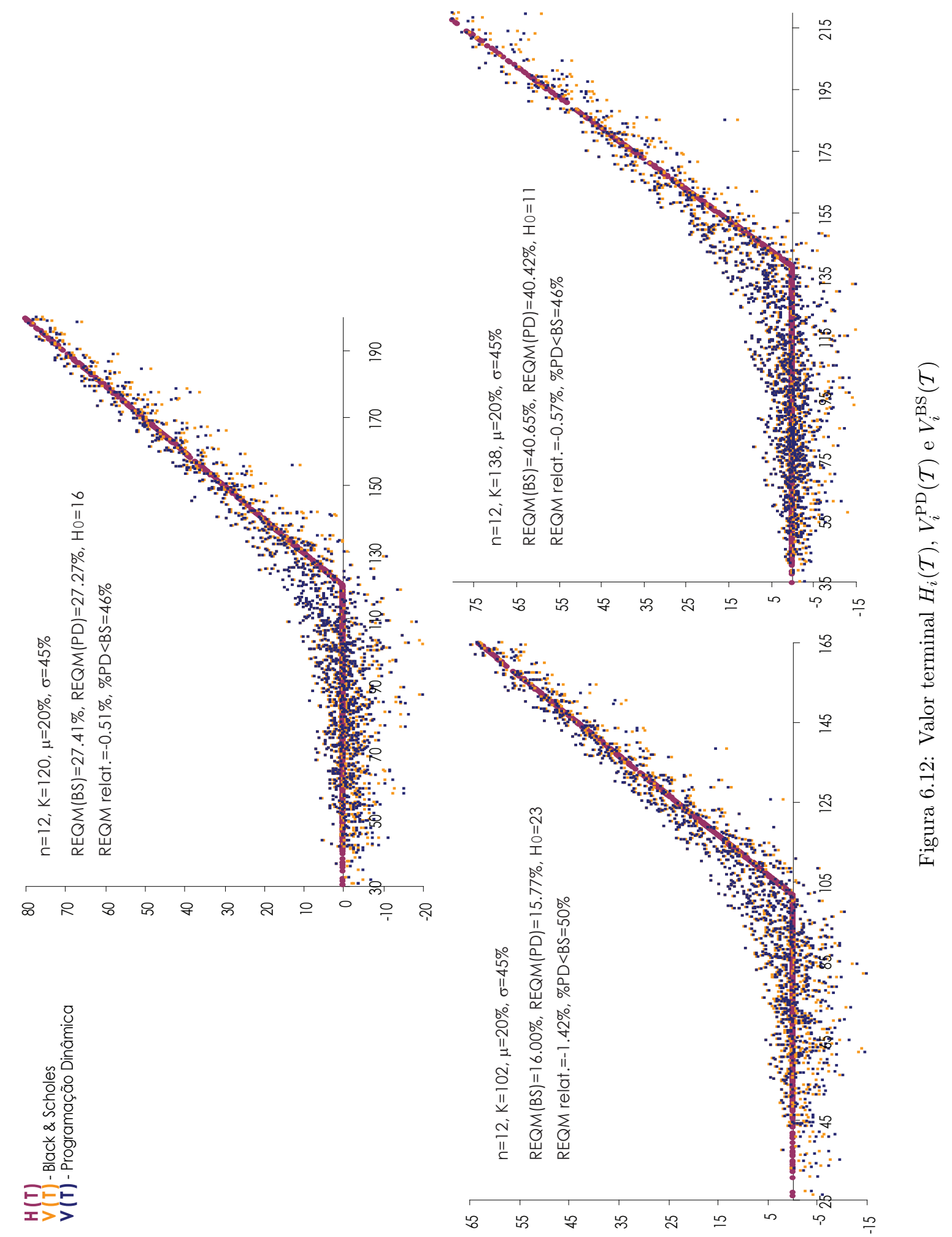


\subsection{Discussão dos Resultados}

Os gráficos da Figura (6.13) permitem fazer uma análise comparativa da raiz quadrada do erro quadrático médio de hedging (REQM) obtido nas simulações ilustradas acima quando usamos a estratégia de hedging derivada da técnica de controle ótimo estocástico (programação dinâmica estocástica), e quando usamos a estratégia de hedging derivada do modelo de Black \& Scholes (deltaneutra). A variável mostrada nos gráficos a seguir e que nos permite fazer essa análise comparativa é a REQM relat., definida no item anterior (Equação (6.11)). 

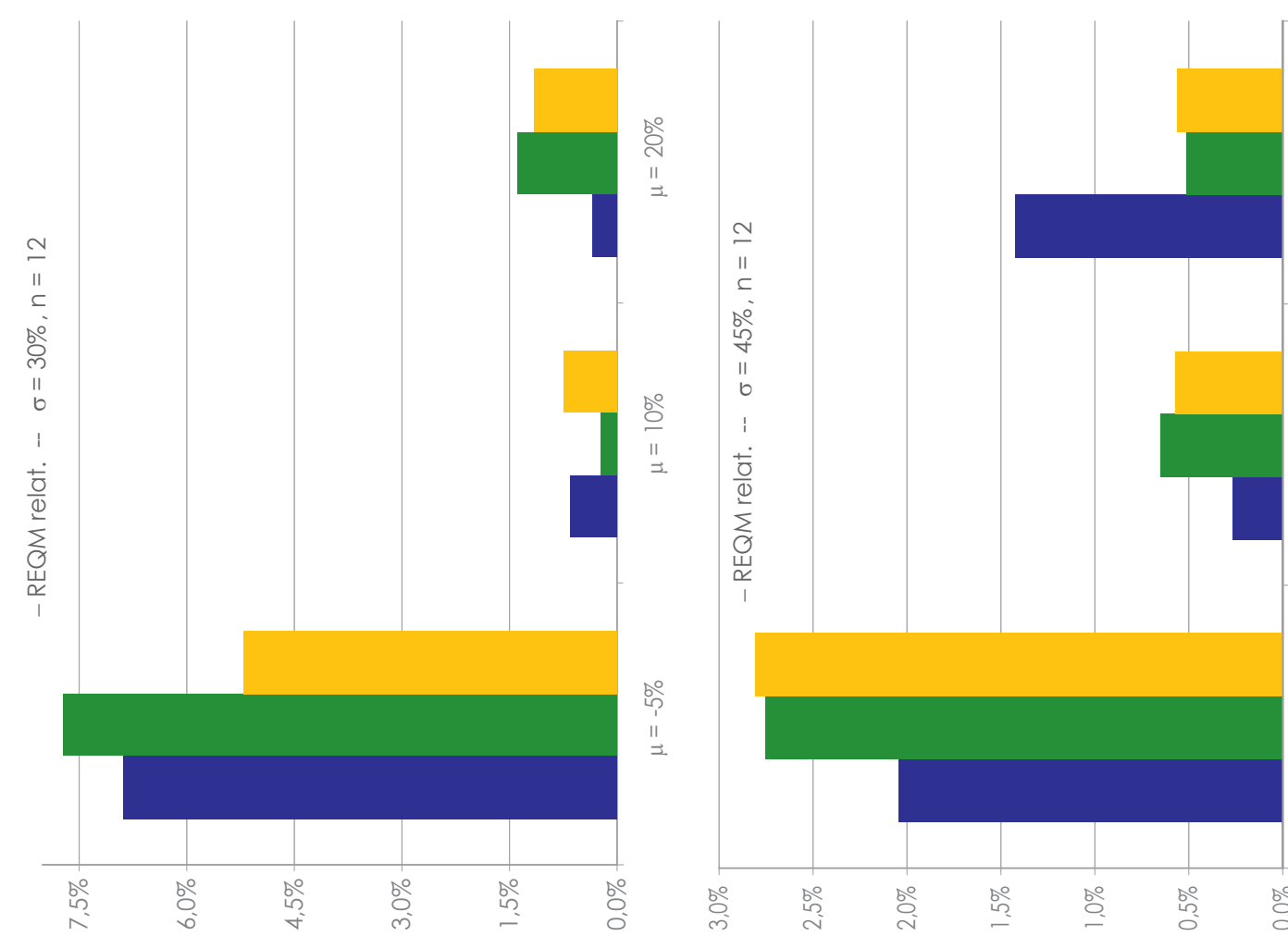

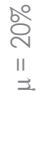

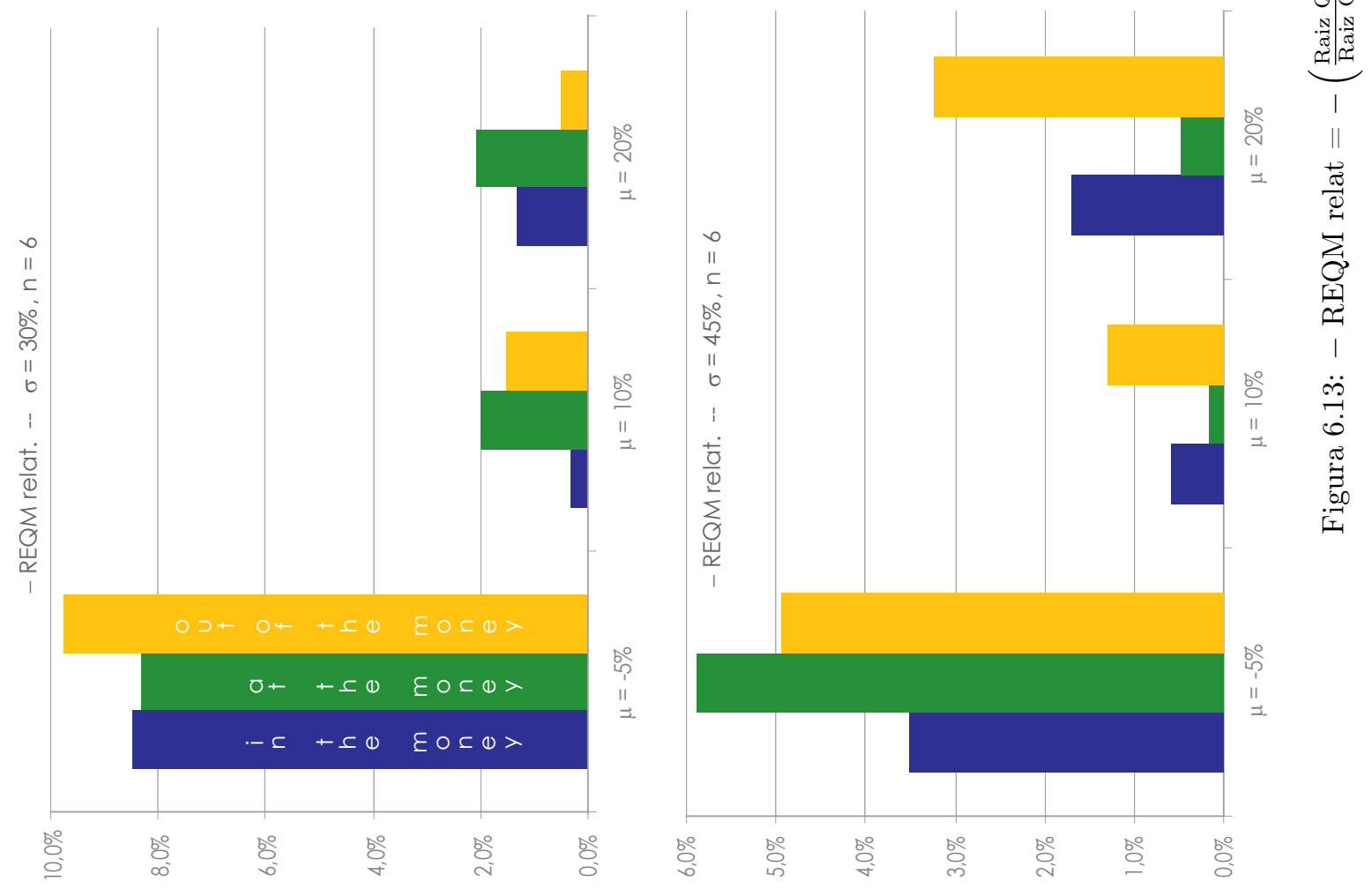


Por esses gráficos, constatamos que a estratégia de hedging derivada da técnica de controle ótimo estocástico (chamada, a partir de agora, de estratégia $\mathrm{PD}$ ) realmente provê resultados consistentemente melhores do que os resultados obtidos a partir da estratégia de hedging derivada do modelo de Black \& Scholes (chamada, a partir de agora, de estratégia BS). Em outras palavras, o erro quadrático médio de hedging obtido com as estratégias PD é inferior a esse erro obtido com as estratégias BS.

$\mathrm{Na}$ maioria dos casos analisados, o desempenho da estratégia PD, embora consistentemente melhor que o da estratégia BS, não é significativamente melhor a ponto de haver comprovação estatística relevante de tal superioridade. Entretanto, essa superioridade é obviamente esperada por construção, uma vez que a estratégia PD foi obtida como solução do problema teórico de minimização do erro quadrático esperado de hedging (Equação (6.1)).

Pelos gráficos, podemos notar que, a princípio, a superioridade da estratégia PD com relação à estratégia BS não apresenta dependência consistente e relevante com relação ao moneyness das opções, i.e., não apresenta dependência consistente e importante ao fato de as opções estarem dentro do dinheiro (in the money), fora do dinheiro (out of the money) ou no dinheiro (at the money).

Em todos os casos analisados, os melhores resultados para o desempenho da estratégia $\mathrm{PD}$ com relação à estratégia BS foram obtidos para $\mu=-5 \%$ a.a..

A estratégia BS é completamente independente da taxa de retorno esperada $\mu$ do ativo base, pois o modelo de Black \& Scholes assume que os hedgings podem ser feitos continuamente ao longo do tempo, eliminando completamente o risco de flutuações estocásticas no preço do ativo base [3, 35]. A estratégia PD, por sua vez, depende explicitamente de $\mu$, como se pode ver pelas equações do Teorema 5.5. Assim, é razoável esperarmos que a superioridade da estratégia PD em relação à estratégia BS apresente dependência em relação a $\mu$. Entretanto, os efeitos da forma com que ocorre esta dependência não estão claros até o presente momento, e podem ser objeto de maiores estudos no futuro. $\mathrm{O}$ fato aqui observado é que o desempenho da estratégia PD foi tanto melhor quanto menor a taxa de retorno esperada $\mu$ do ativo base.

De forma geral, a estratégia PD apresentou melhor desempenho em relação à estratégia BS quando o número de rebalanceamentos foi menor, ou seja, quando $n=6$. Isso de fato era esperado, pois o modelo de Black \& Scholes assume que os rebalanceamentos são refeitos continuamente. O modelo desenvolvido nesta tese, por sua vez, considera que os rebalanceamentos são refeitos em instantes pertencentes a um domínio discreto do tempo. Assim, quanto menor o número de rebalanceamentos, mais nos distanciamos das hipóteses do modelo de Black \& Scholes [3, 16, 35], e pior é o resultado da estratégia BS em relação à estratégia PD.

Embora a estratégia PD tenha sido consistentemente mais eficiente que a estratégia BS, devemos destacar dois fatos.

O primeiro é que a magnitude dos erros de hedging (medidos por REQM, raiz quadrada do erro quadrático médio de hedging) para as duas estratégias varia de um valor mínimo em torno de $6 \%$ com relação a $H(0)$, a um valor máximo em torno de $60 \%$ com relação a $H(0)$. Assim, os erros de hedging podem ser realmente bastante significativos. Os gráficos da Figura (6.14) ilustram os erros de hedging (i.e., REQM(PD) e REQM(BS)) para as duas estratégias. Uma análise aprofundada do comportamento desses erros não faz parte do escopo desta tese, e pode ser objeto de estudo futuro. A propósito, qualquer estudo do 
comportamento dos erros de hedging para a estratégia PD deve começar pela Equação (3.22). Além disso, conforme apresentado no Teorema 3.3, $\overline{\mathcal{J}}_{0, \mathcal{T}}(0)=$ $\delta(0)$ corresponde ao erro quadrático de hedging esperado em $\tau=0$.

O segundo fato a ser destacado é o bom desempenho da estratégia BS, ainda que consistentemente superada pela estratégia PD. Podemos atribuir esse desempenho a pelo menos dois fatores fundamentais. O primeiro deles é que as simulações foram feitas em condições que não se distanciam muito das hipóteses do modelo de Black \& Scholes. Mais especificamente, de todas as hipóteses do modelo, a única violada nessas simulações foi a de que aqui os rebalanceamentos do portfólio foram feitos de forma discreta, e não de forma contínua, como requer o modelo. O segundo fator responsável pelo bom desempenho da estratégia BS é a robustez do modelo de Black \& Scholes [6, 8]. Devido a essa robustez, o modelo funciona muito bem mesmo que diversas de suas hipóteses sejam violadas, fato que certamente é responsável pela sua grande popularidade entre os profissionais do mercado financeiro.

Finalmente, é importante observar que, ainda que o desempenho da estratégia PD seja apenas pouco melhor que o desempenho da estratégia BS, no mercado financeiro real, os portfólios de opções podem, em algumas instituições com participação ativa nesse mercado, chegar a valores da ordem de bilhões de dólares. Assim, uma pequena melhora no desempenho da estratégia de hedging pode significar, nesses casos, uma economia de centenas de milhares de dólares, o que certamente justifica os custos de implementação da estratégia PD. 


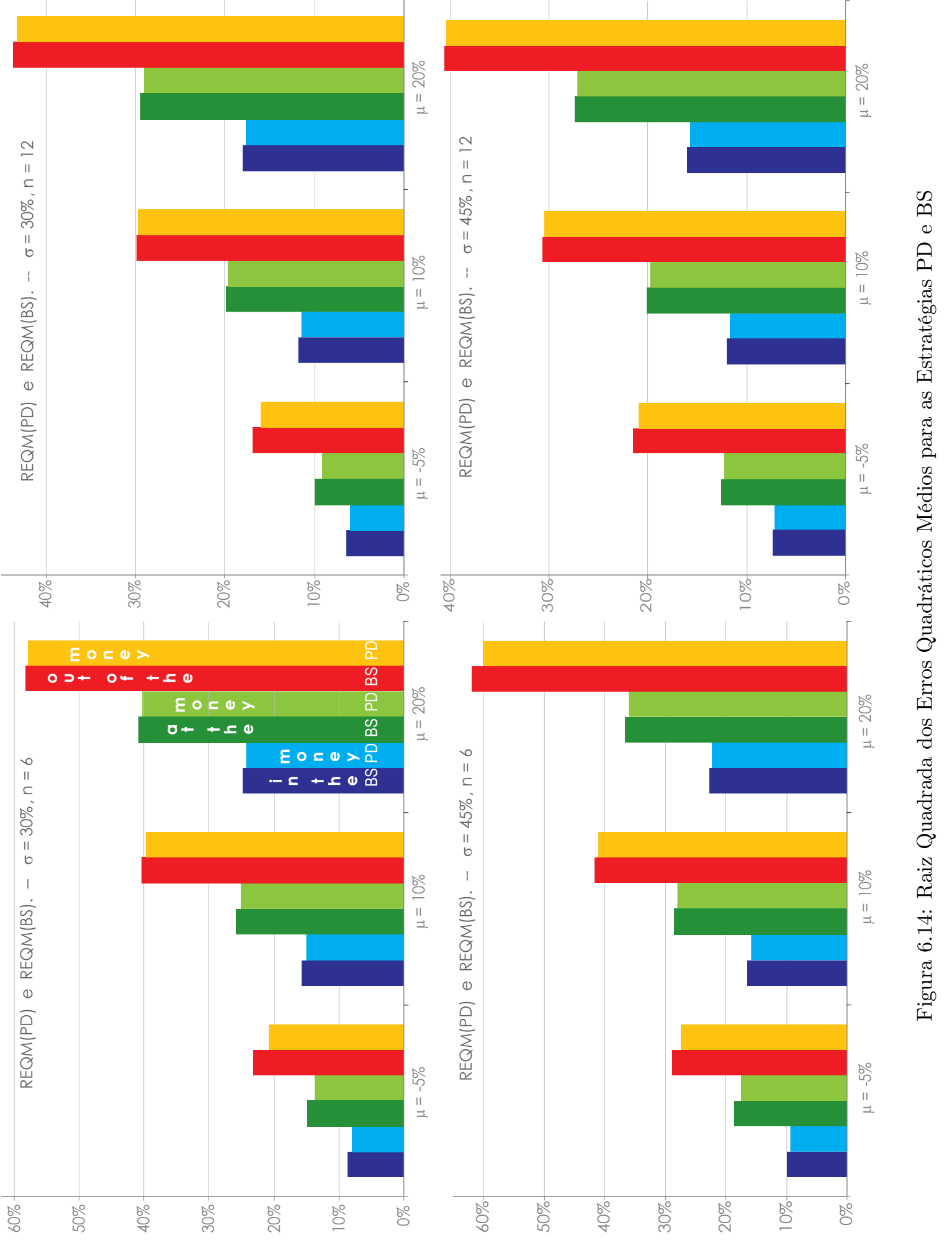




\section{Capítulo 7}

\section{Conclusões e Pesquisas Futuras}

Neste item, inicialmente apresentamos as conclusões desta tese, reiterando os objetivos desta tese, os resultados obtidos e suas implicações. Em seguida, apresentamos nossas recomendações para pesquisas futuras, identificando melhorias possíveis neste trabalho, bem como questões que podem ser objeto de estudo mais aprofundado e desenvolvimento no futuro.

\subsection{Conclusões}

O objetivo desta tese era o de explorar e resolver o problema de hedging de mínima variância de um contrato derivativo não-replicável usando métodos de programação dinâmica estocástica em tempo discreto, com espaço de estados contínuo.

A estratégia adotada para atingirmos esse objetivo foi a de, inicialmente, desenvolver um modelo geral para o apreçamento e hedging de derivativos de característica européia com payoffs completamente genéricos, possivelmente dependentes dos $n$ ativos de risco existentes no mercado, ou da trajetória seguida pelos preços desses ativos. O mercado adotado como modelo opera em tempo discreto e em diferentes modos, que foram matematicamente representados por uma cadeia de Markov. O modelo de apreçamento e hedging desenvolvido não depende de qualquer hipótese sobre a dinâmica dos preços dos ativos de risco existentes no mercado, e se aplica igualmente bem a modelos com espaço de estado discreto ou contínuo. Em outras palavras, a dinâmica dos preços dos ativos de risco pode ser representada por um grid de pontos (i.e., por uma árvore), ou por uma equação diferencial estocástica. Vale dizer, ainda, que esse modelo desenvolvido também é capaz de tratar situações em que o ativo livre de risco existente no mercado possui comportamento estocástico. Esse modelo foi apresentado no Capítulo 3 e foi a primeira contribuição original desta tese.

Em termos práticos, um modelo em que o mercado opere em diferentes modos significa que, a cada instante do domínio do tempo, o comportamento do mercado pode ser descrito por um modelo matemático $\mathcal{G}_{i}, i \in\{1, \ldots, N\}$, onde cada um desses modelos pode conferir ao mercado elementos como, por exemplo: (1) ativos de risco cujos retornos apresentem volatilidade estocástica, 
(2) taxas de retornos estocásticas para os ativos de risco, (3) taxas de juros estocásticas descritas por processos com parâmetros variáveis.

Assim, o modelo desenvolvido provê uma estrutura geral que se pode empregar para o apreçamento e hedging de derivativos de característica européia, segundo diversos modelos de funcionamento do mercado, quando consideramos o tempo como pertencente a um domínio discreto (que, como explicado antes, é o caso que melhor reflete a realidade dos processos de tomada de decisão de investimento).

Após termos desenvolvido esse modelo geral, fizemos algumas simplificações em sua estrutura. Mais especificamente, a primeira simplificação correspondeu a adotarmos um modelo de mercado que opere em apenas um modo. Em outras palavras, eliminamos a cadeia de Markov que representava os diferentes modos de operação do mercado. Mostramos, então, que essa primeira simplificação leva o nosso modelo a resultados que coincidem com os obtidos por Černý [5]. Dessa forma, mostramos que o modelo proposto por Černý pode ser entendido como um caso particular do modelo desenvolvido nesta tese.

A próxima simplificação que fizemos correspondeu a restringir nosso modelo ao caso em que o mercado opere em um único modo e que contenha apenas um ativo de risco e um outro livre de risco. No Capítulo 4, aplicamos esse modelo de apreçamento e hedging ao caso em que os retornos do ativo de risco assumem valores num espaço de estados contínuo, sendo descritos por um processo de difusão descontínuo (i.e., com saltos, também chamado de jump diffusion process) e com parâmetros constantes (constant investment opportunity set).

Os resultados obtidos no Capítulo 4 correspondem a um importante avanço do modelo geral desenvolvido no Capítulo 3 em direção à prática dos mercado financeiros, pois incorporam um elemento bastante concreto da estrutura de um mercado financeiro real, a saber, a dinâmica dos retornos do ativo de risco descrita segundo um processo de difusão com saltos num espaço de estados contínuo. Uma importante contribuição desses resultados obtidos nesta tese à teoria de apreçamento e hedging de derivativos é que eles estabelecem a estrutura a partir da qual se podem obter expressões analíticas fechadas para: (1) o preço de um derivativo com um payoff qualquer (i.e., completamente genérico), desde que de característica européia e com tratabilidade matemática, e (2) o controle ótimo da carteira que faz o hedging de mínima variância desse derivativo, num modelo de mercado em que o tempo é discreto, o espaço de estados é contínuo e a dinâmica dos retornos do ativo de risco é descrita segundo um processo de difusão com saltos, atendendo assim às premissas que melhor refletem as condições de funcionamento do mercado financeiro real. No caso de não haver tratabilidade matemática suficiente, impossibilitando a obtenção de expressões analíticas fechadas, essa mesma estrutura que apresentamos no Capítulo 4 provê os meios para a execução de simulações numéricas de Monte Carlo [17, 12], em todas as suas variações, permitindo então obter os mesmos resultados descritos acima, porém de forma numérica.

Devido à sua tratabilidade matemática e à sua ampla difusão no mercado financeiro, no Capítulo 5 aplicamos os resultados gerais obtidos no Capítulo 4 para o caso de uma opção vanilla de compra européia, obtendo expressões explícitas fechadas para o preço dessa opção e para o controle necessário à execução do hedging desse derivativo. Os resultados obtidos neste capítulo são muito interessantes, não só pela originalidade, mas também porque correspondem a um paralelo mais realista e mais correto, do ponto de vista prático, do resultado 
apresentado por Merton [20], onde o modelo adotado para o mercado é contínuo com relação ao tempo e ao espaço de estados.

Infelizmente, o desempenho desse modelo na prática não pôde ser testado aqui por motivo de limitação no tempo de desenvolvimento desta tese. Em outras palavras, não foram feitas simulações numéricas usando os resultados mencionados acima.

Ainda no Capítulo 5, particularizamos os resultados obtidos para a op̧̧ão de compra vanilla européia mencionados acima ao caso em que os retornos do ativo de risco são descritos por um processo de difusão sem saltos, i.e., por um processo browniano (ou de Wiener). Esses resultados também são interessantes, não só pela originalidade, mas também porque correspondem a um paralelo mais realista e mais correto, do ponto de vista prático, do resultado apresentado por Black \& Scholes [3], onde o modelo adotado para o mercado é contínuo com relação ao tempo e ao espaço de estados.

Para esse modelo, as simulações numéricas foram realizadas. Essas simulações nos permitiram, então, avaliar o desempenho, na prática, do hedging de uma opção vanilla de compra européia segundo o modelo desenvolvido nesta tese para o caso em que os retornos do ativo de risco são descritos por um movimento browniano. O desempenho do modelo aqui desenvolvido foi comparado ao desempenho da estratégia de hedging derivada do modelo de Black \& Scholes, chamada estratégia delta-neutra. Os resultados dessa comparação foram discutidos extensamente no Capítulo 6.

De forma bem resumida, devemos destacar dois pontos fundamentais como conclusão final para essas simulações. O primeiro deles é que, caso o portfólio de opções a ser protegido por uma estratégia dinâmica de hedging seja extremamente vultoso, aí sim o modelo aqui proposto apresenta grande vantagem em relação à estratégia delta-neutra, que é simples e de fácil implementação. Caso o portfólio de derivativos cujo risco deva ser minimizado corresponda a um volume financeiro pequeno, a melhor decisão é usar a estratégia derivada do modelo de Black \& Scholes, i.e., a estratégia delta-neutra.

O segundo ponto é que, para qualquer modelo que se use, os erros quando do hedging de uma opção não são triviais. Pelo contrário, dependendo da situação (como se pôde ver claramente nos gráficos apresentados), eles podem ser muito elevados, o que realmente requer que o vendedor de tal produto cobre um prêmio do comprador suficiente para eventualmente compensar suas perdas financeiras na maturidade do contrato.

Como último comentário, é interessante notar nos gráficos que mostram REQM(PD) e REQM(BS) (i.e., a raiz quadrada dos erros quadráticos médios para as estratégias PD e BS) que o erro de hedging é maior para a opção fora do dinheio, qualquer que seja a situação analisada. Isto pode ser mais uma razão, entre outras várias [33, 7], que contribui para a existência do smile de volatilidade.

\subsection{Pesquisas Futuras}

A literatura da área de finanças quantitativas é extremamente rica com relação ao estudo do problema de hedging de mínima variância em tempo e estado contínuos. No caso de tempo e espaço de estados discretos, o número de trabalhos que abordaram esse problema até o presente momento ainda é relativamente 
pequeno. Contudo, com relação ao caso em que o domínio do tempo é discreto e o espaço de estados é contínuo, parece que esse ainda é um campo relativamente bastante inexplorado (já que não encontramos referências sobre o assunto). Apesar de esse último problema ter sido o objeto de estudo desta tese, o tema, evidentemente, está muito longe do esgotamento.

Contudo, como explicado no Item 1.2, esse parece, e deveria, ser o problema de maior interesse dos profissionais que realmente atuam no mercado financeiro negociando e gerindo o risco de contratos derivativos, já que essa situação é a que mais se aproxima de um modelo apropriado para descrever o problema real de hedging desse tipo de instrumento financeiro.

Vultosas somas de dinheiro estão envolvidas nas aplicações práticas desse problema. De fato, um controle mais eficaz do risco existente numa carteira de derivativos pode evitar perdas estocásticas significativas devido a flutuações nos preços dos ativos base dos quais depende esta carteira. Além disso, agentes financeiros que disponham de melhores técnicas para gestão desse tipo de risco podem ser mais competitivos na estruturação e geração de negócios junto às suas contrapartes (clientes e mercado), aumentando sua receita e expandindo sua influência no mercado. Portanto, o interesse sobre técnicas mais apuradas de hedging de derivativos continuará, cada vez mais, a merecer atenção por parte de acadêmicos e profissionais do mercado.

Nesse contexto, os resultados desta tese, que estabelecem uma estrutura teórica para o apreçamento e hedging de derivativos num modelo mais próximo da realidade do mercado financeiro, podem ser futuramente estendidos e aprimorados, contribuindo ainda mais com a busca de soluções satisfatórias para as questões apontadas anteriormente. Citamos a seguir alguns possíveis futuros desenvolvimentos:

1. Volatilidade estocástica. O modelo geral desenvolvido no Capítulo 3, onde o mercado pode apresentar diferentes modos de operação, acomoda, como já comentado anteriormente, (1) ativos de risco cujos retornos apresentem volatilidade estocástica, (2) taxas de retornos estocásticas para os ativos de risco, (3) taxas de juros estocásticas descritas por processos com parâmetros variáveis. Desses, o ponto mais significativo parece ser a inclusão dos efeitos de retornos com volatilidade estocástica aos resultados obtidos, especialmente às soluções fechadas apresentadas no Capítulo 5, já que sabemos que a volatilidade é uma variável à qual os preços das opções apresentam bastante sensibilidade. Assim, simplesmente usando-se a estrutura teórica estabelecida nesta tese, é possível se estabelecerem dinâmicas estocásticas para a volatilidade e, de forma análoga ao que fizemos nesta tese, acrescentar aos resultados obtidos os efeitos dessas dinâmicas aos preços e à forma de controle dos derivativos estudados.

2. Critérios de otimização mais gerais. O modelo de hedging desenvolvido nesta tese adotou um critério de otimização quadrático para considerar os erros que se desejava minimizar. Uma extensão futura bastante interessante seria a de considerar critérios de otimização diferentes, como, por exemplo, da forma $J(x)=a x^{2}+b x^{4}$, sendo $x$ o erro de hedging, e $a$ e $b$ constantes pré-determinadas. Um critério desse tipo tem a vantagem de punir mais rigorosamente os erros de hedging de maior valor, levando o modelo a propor estratégias de controle que evitem grandes perdas gera- 
das por eventos extremos. A presença de um termo de segunda ordem tem a função de assegurar que termos menores sejam também considerados.

3. Simulações de Monte Carlo. Como descrito anteriormente, a estrutura teórica estabelecida nesta tese para o apreçamento e hedging de derivativos pode ser usada quando não existe tratabilidade matemática suficiente para se encontrarem expressões analíticas fechadas para o preço e para o controle desses derivativos. Assim, considerar o caso de derivativos com payoffs não-tratáveis analiticamente, especialmente aqueles que apresentem dependência da trajetória dos ativos base, parece também uma interessante possibilidade de extensão deste trabalho. Nesses casos, os resultados e a eficiência do modelo aqui proposto poderiam ser comparados aos produzidos pelas técnicas atuais de apreçamento e hedging.

4. Retornos com dinâmicas mais gerais. Os resultados analíticos fechados obtidos aqui consideram que os retonos do ativo de risco são dados por processos de difusão gaussianos com saltos descritos por um processo composto de Poisson. Uma extensão possível seria a de se considerarem distribuições não-gaussianas, com caudas pesadas, por exemplo, para descrever o processo de difusão dos retornos do ativo de risco. Para os saltos, poderiam ser considerados processos mais gerais empregados, por exemplo, na teoria de valores extremos [10].

5. Custos de transação. Uma extensão óbvia aos modelos aqui propostos seria a inclusão de custos de transação. Esses custos são importantes, pois contribuem para aumentar o erro de hedging, já que, a cada rebalanceamento (i.e., a cada ação de controle), reduzem o valor do portfólio cujo objetivo é reproduzir o derivativo não-replicável analisado. A dificuldade enfrentada aqui é que o modelo se tornará, salvo melhor juízo, não-linear.

6. Freqüência ótima de rebalanceamento. Uma vez inseridos custos de transação no modelo, poderia ser incluída uma nova variável à função sendo otimizada: a freqüência de rebalanceamento, ou seja, a freqüência com que são tomadas as decisões de controle. Evidentemente, quanto maior esta freqüência, menores tendem a ser os desvios da estratégia de controle em relação ao derivativo em questão. Contudo, nesse caso, crescem também os custos de transação, o que, como explicado anteriormente, aumenta o erro de hedging. Por outro lado, se as decisões de controle forem menos freqüentes, embora os custos de transação sejam menores, maiores tendem a ser os desvios da estratégia de controle em relação ao derivativo que se deseja replicar. Claramente, então, existe uma política ótima também com relação à freqüência de hedging.

Além destas, uma outra extensão óbvia e trivial, e que certamente será feita pelo próprio autor, é a de se realizarem simulações numéricas totalmente análogas às já apresentadas aqui, usando, entretando, o modelo de apreçamento e hedging desenvolvido nesta tese em que os retornos são descritos por um processo de difusão com saltos (modelo apresentado no Item 5.1). Como dito anteriormente, essas simulações não foram feitas aqui por restrições de tempo. 


\section{Apêndice A}

\section{Demonstrações}

Lema A.1. Consideremos o problema:

$$
U=\min _{u} \sum_{j} p_{i, j} E^{P}\left(\left(Y_{j}+X_{j}^{*} u\right)^{2}+\delta_{j}\right),
$$

onde $p_{i, j} \geq 0$. Assumamos que:

$$
\sum_{j} p_{i, j} E^{P}\left(X_{j} X_{j}^{*}\right)>0 .
$$

Então, o u ótimo, $\bar{u}$, é dado por:

$$
\bar{u}=-\left[\sum_{j} p_{i, j} E^{P}\left(X_{j} X_{j}^{*}\right)\right]^{-1}\left(\sum_{j} p_{i, j} E^{P}\left(X_{j} Y_{j}\right)\right)
$$

$$
U=U_{0}+\sum_{j} p_{i, j} E^{P}\left(\delta_{j}\right)
$$

onde,

$$
\begin{aligned}
U_{0} & =\sum_{j} p_{i, j} E^{P}\left(Y_{j}^{2}\right)- \\
& \left(\sum_{j} p_{i, j} E^{P}\left(Y_{j} X_{j}^{*}\right)\right)\left[\sum_{l} p_{i, l} E^{P}\left(X_{l} X_{l}^{*}\right)\right]^{-1}\left(\sum_{j} p_{i, j} E^{P}\left(X_{j} Y_{j}\right)\right) \geq 0 .
\end{aligned}
$$

Demonstração. Temos que:

$$
\begin{aligned}
U=\min _{u} \sum_{j} p_{i, j} E^{P} & \left(\left(Y_{j}+X_{j}^{*} u\right)^{2}+\delta_{j}\right)= \\
& \underbrace{\min _{u} \sum_{j} p_{i, j} E^{P}\left(\left(Y_{j}+X_{j}^{*} u\right)^{2}\right)}_{U_{0}}+\sum_{j} p_{i, j} E^{P}\left(\delta_{j}\right) .
\end{aligned}
$$


Evidentemente, $U_{0} \geq 0$ e:

$$
\begin{gathered}
\sum_{j} p_{i, j} E^{P}\left(\left(Y_{j}+X_{j}^{*} u\right)^{2}\right)= \\
\sum_{j} p_{i, j} E^{P}\left(Y_{j}^{2}+2 Y_{j} X_{j}^{*} u+u^{*} X_{j}^{*} X_{j} u\right)= \\
\sum_{j} p_{i, j} E^{P}\left(Y_{j}^{2}\right)+2 \sum_{j} p_{i, j} E^{P}\left(Y_{j} X_{j}^{*}\right) u+u^{*} \sum_{j} p_{i, j} E^{P}\left(X_{j} X_{j}^{*}\right) u .
\end{gathered}
$$

Derivando a expressão acima em relação a $u$ e igualando a zero:

$$
\sum_{j} p_{i, j} E^{P}\left(X_{j} X_{j}^{*}\right) \bar{u}=-\sum_{j} p_{i, j} E^{P}\left(X_{j} Y_{j}\right)
$$

Isolando $\bar{u}$ :

$$
\bar{u}=-\left[\sum_{j} p_{i, j} E^{P}\left(X_{j} X_{j}^{*}\right)\right]^{-1}\left(\sum_{j} p_{i, j} E^{P}\left(X_{j} Y_{j}\right)\right) .
$$

Derivando mais uma vez em relação a $u$, temos:

$$
\frac{d^{2}}{d u^{2}}\left(\sum_{j} p_{i, j} E^{P}\left(\left(Y_{j}+X_{j}^{*} u\right)^{2}+\delta_{j}\right)\right)=\sum_{j} p_{i, j} E^{P}\left(X_{j} X_{j}^{*}\right)>0
$$

por hipótese, o que mostra que $\bar{u}$ é de fato a solução ótima que minimiza a função objetivo.

Substituindo $\bar{u}$ na função objetivo, obtemos:

$$
\begin{gathered}
U_{0}=\sum_{j} p_{i, j} E^{P}\left(Y_{j}^{2}\right)+2\left(\sum_{j} p_{i, j} E^{P}\left(Y_{j} X_{j}^{*}\right)\right) u+u^{*} B u= \\
\sum_{j} p_{i, j} E^{P}\left(Y_{j}^{2}\right)-2\left(\sum_{j} p_{i, j} E^{P}\left(Y_{j} X_{j}^{*}\right)\right) B^{-1}\left(\sum_{j} p_{i, j} E^{P}\left(X_{j} Y_{j}\right)\right)+ \\
\left(B^{-1}\left(\sum_{j} p_{i, j} E^{P}\left(X_{j} Y_{j}\right)\right)\right)^{*} B B^{-1}\left(\sum_{j} p_{i, j} E^{P}\left(X_{j} Y_{j}\right)\right), \\
B=\sum_{j} p_{i, j} E^{P}\left(X_{j} X_{j}^{*}\right) .
\end{gathered}
$$

Simplificando: 


$$
\begin{aligned}
& U_{0}=\sum_{j} p_{i, j} E^{P}\left(Y_{j}^{2}\right)- \\
& 2\left(\sum_{j} p_{i, j} E^{P}\left(Y_{j} X_{j}^{*}\right)\right)\left[\sum_{j} p_{i, j} E^{P}\left(X_{j} X_{j}^{*}\right)\right]^{-1}\left(\sum_{j} p_{i, j} E^{P}\left(X_{j} Y_{j}\right)\right)+ \\
& \left(\sum_{j} p_{i, j} E^{P}\left(Y_{j} X_{j}^{*}\right)\right)\left[\sum_{j} p_{i, j} E^{P}\left(X_{j} X_{j}^{*}\right)\right]^{-1}\left(\sum_{j} p_{i, j} E^{P}\left(X_{j} Y_{j}\right)\right) .
\end{aligned}
$$

Portanto $U$ é igual a:

$$
\begin{aligned}
U=U_{0}+\sum_{j} p_{i, j} E^{P}\left(\delta_{j}\right) & =\sum_{j} p_{i, j} E^{P}\left(Y_{j}^{2}\right)- \\
\left(\sum_{j} p_{i, j} E^{P}\left(Y_{j} X_{j}^{*}\right)\right)\left[\sum_{j} p_{i, j} E^{P}\left(X_{j} X_{j}^{*}\right)\right]^{-1}\left(\sum_{j} p_{i, j} E^{P}\left(X_{j} Y_{j}\right)\right)+ & \sum_{j} p_{i, j} E^{P}\left(\delta_{j}\right) .
\end{aligned}
$$

Lema A.2. Suponhamos que $k_{j}>0, j=1, \ldots, N$, e que não existam oportunidades de arbitragem. Então $\vec{k}_{i}>0$, onde:

$$
\begin{gathered}
M_{\theta(\tau)}=\sum_{j} p_{\theta(\tau), j} E_{\tau}^{P}\left\{k_{j} \Delta X_{\theta(\tau)} \Delta X_{\theta(\tau)}^{*}\right\}, \\
\bar{k}_{j}=a_{\theta(\tau)}(\tau)^{2}\left[\sum_{j} p_{\theta(\tau), j} E^{P}\left(k_{j}\right)-\right. \\
\left.\left(\sum_{j} p_{\theta(\tau), j} E^{P}\left(k_{j} \Delta X_{\theta(\tau)}^{*}\right)\right) M_{\theta(\tau)}^{-1}\left(\sum_{j} p_{\theta(\tau), j} E^{P}\left(k_{j} \Delta X_{\theta(\tau)}\right)\right)\right] .
\end{gathered}
$$

Demonstração. Mostremos primeiramente que $\bar{k}_{i} \geq 0$. Consideremos o problema:

$$
\mathcal{J}=\min _{u} \sum_{j} p_{\theta(\tau), j} E_{\tau}^{P}\left(\left(a_{\theta(\tau)} \sqrt{k_{j}}-a_{\theta(\tau)} \sqrt{k_{j}} \Delta X_{\theta(\tau)}^{*} u\right)^{2}\right)
$$

Pelo Lema A.1, a solução ótima desse problema é:

$$
\begin{array}{r}
\bar{u}=-\left[\sum_{j} p_{\theta(\tau), j} E^{P}\left(k_{j} \Delta X_{\theta(\tau)} \Delta X_{\theta(\tau)}^{*}\right)\right]^{-1}\left(\sum_{j} p_{\theta(\tau), j} E^{P}\left(k_{j} \Delta X_{\theta(\tau)}\right)\right)= \\
M_{\theta(\tau)}^{-1}\left(\sum_{j} p_{\theta(\tau), j} E^{P}\left(k_{j} \Delta X_{\theta(\tau)}\right)\right)
\end{array}
$$




$$
\begin{aligned}
& \mathcal{J}=k_{\theta(\tau)}=a_{\theta(\tau)}(\tau)^{2}\left[\sum_{j} p_{\theta(\tau), j} E^{P}\left(k_{j}\right)-\right. \\
& \left.\left(\sum_{j} p_{\theta(\tau), j} E^{P}\left(k_{j} \Delta X_{\theta(\tau)}^{*}\right)\right) M_{\theta(\tau)}^{-1}\left(\sum_{j} p_{\theta(\tau), j} E^{P}\left(k_{j} \Delta X_{\theta(\tau)}\right)\right)\right] \geq 0 .
\end{aligned}
$$

Ou seja, $\bar{k}_{\theta(\tau)} \geq 0$. Suponha que $\bar{k}_{\theta(\tau)}=0$. Então:

$$
\begin{aligned}
& \sum_{j} p_{\theta(\tau), j} E_{\tau}^{P}\left(\left(a_{\theta(\tau)} \sqrt{k_{j}}-a_{\theta(\tau)} \sqrt{k_{j}} \Delta X_{\theta(\tau)}^{*} \bar{u}\right)^{2}\right)= \\
& E_{\tau}^{P}\left(a_{\theta(\tau)}(\tau)^{2} k_{\theta(\tau+1)}\left(1-\Delta X_{\theta(\tau)}^{*} \bar{u}\right)^{2} \mid \mathcal{F}_{\tau}\right)=0
\end{aligned}
$$

Isto é:

$$
a_{\theta(\tau)}(\tau)^{2} k_{\theta(\tau+1)}\left(1-\Delta X_{\theta(\tau)}^{*} \bar{u}\right)=0 \quad \text { a.s. }
$$

Como $k_{j}>0, j=1, \ldots, N$ e $a_{\theta(\tau)}(\tau)^{2}>0$, temos que:

$$
\Delta X_{\theta(\tau)}^{*} \bar{u}=1 \quad \text { a.s. }
$$

A condição de ausência de arbitragem assegura que existe uma medida de probabilidade $\pi$ tal que:

$$
E^{\pi}\left(S_{\theta(\tau)}(\tau+1) \mid \mathcal{F}_{\tau}\right)=S(\tau)\left(1+r_{\theta(\tau)}(\tau)\right)=S(\tau) a_{\theta(\tau)}(\tau) .
$$

Como:

$$
\Delta X_{\theta(\tau)}=\frac{S_{\theta(\tau)}(\tau+1)}{S_{\theta(\tau), 0}(\tau+1)}-\frac{S(\tau)}{S_{0}(\tau)}
$$

e

$$
S_{\theta(\tau), 0}(\tau+1)=S_{0}(\tau) a_{\theta(\tau)}(\tau),
$$

podemos escrever:

$$
\begin{aligned}
E^{\pi}\left(\Delta X_{\theta(\tau)} \mid \mathcal{F}_{\tau}\right)= & E^{\pi}\left(\frac{S_{\theta(\tau)}(\tau+1)}{S_{0}(\tau) a_{\theta(\tau)}(\tau)}-\frac{S(\tau)}{S_{0}(\tau)} \mid \mathcal{F}_{\tau}\right)= \\
& E^{\pi}\left(\frac{S_{\theta(\tau)}(\tau+1)}{S_{0}(\tau) a_{\theta(\tau)}(\tau)} \mid \mathcal{F}_{\tau}\right)-E^{\pi}\left(\frac{S(\tau)}{S_{0}(\tau)} \mid \mathcal{F}_{\tau}\right)=0
\end{aligned}
$$

Neste caso, $E^{\pi}\left(\Delta X_{\theta(\tau)}^{*} \bar{u} \mid \mathcal{F}_{\tau}\right)=0$, em contradição com $\Delta_{\theta(\tau)}^{*} \bar{u}=1$ a.s.. Portanto: $\bar{k}_{\theta(\tau)}>0$. 
Lema A.3. Consideremos a hipótese do Lema A.2, e

$$
\begin{aligned}
& \mathcal{J}(V(\tau), \theta(\tau), \tau)= \\
& S_{\theta(\tau), 0}(\tau+1)^{2}\left\{\sum_{j} p_{\theta(\tau), j} E_{\tau}^{P}\left\{k_{j}(\tau+1)\left(\frac{V(\tau)}{S_{0}(\tau)}-\frac{H_{j}(\tau+1)}{S_{\theta(\tau), 0}(\tau+1)}\right)^{2}\right\}-\right. \\
& \left(\sum_{j} p_{\theta(\tau), j} E_{\tau}^{P}\left(k_{j}(\tau+1)\left(\frac{V(\tau)}{S_{0}(\tau)}-\frac{H_{j}(\tau+1)}{S_{\theta(\tau), 0}(\tau+1)}\right) \Delta X_{\theta(\tau)}^{*}(\tau+1)\right)\right) M_{\theta(\tau)}^{-1} \\
& \left.\left(\sum_{j} p_{\theta(\tau), j} E_{\tau}^{P}\left(k_{j}(\tau+1)\left(\frac{V(\tau)}{S_{0}(\tau)}-\frac{H_{j}(\tau+1)}{S_{\theta(\tau), 0}(\tau+1)}\right) \Delta X_{\theta(\tau)}(\tau+1)\right)\right)\right\}+ \\
& \sum_{j} p_{\theta(\tau), j} E\left(\delta_{j}\right),
\end{aligned}
$$

onde:

$$
M_{\theta(\tau)}=\sum_{j} p_{\theta(\tau), j} E_{\tau}^{P}\left\{k_{j}(\tau+1) \Delta X_{\theta(\tau)}(\tau+1) \Delta X_{\theta(\tau)}^{*}(\tau+1)\right\} .
$$

Então:

$$
\mathcal{J}(V(\tau), \theta(\tau), \tau)=\bar{K}(V(\tau)-\bar{H})^{2}+\bar{R}-\frac{\bar{U}^{2}}{\bar{K}},
$$

Onde:

$$
\begin{gathered}
\bar{K}=a_{\theta(\tau)}(\tau)^{2}\left[E_{\tau}^{P}\left\{k_{\theta(\tau+1)}(\tau+1)\right\}-\right. \\
\left.E_{\tau}^{P}\left(k_{\theta(\tau+1)}(\tau+1) \Delta X_{\theta(\tau)}^{*}(\tau+1)\right) M_{\theta(\tau)}^{-1} E_{\tau}^{P}\left(k_{\theta(\tau+1)}(\tau+1) \Delta X_{\theta(\tau)}(\tau+1)\right)\right],
\end{gathered}
$$

$$
\begin{gathered}
\bar{H}=\frac{1}{\frac{\bar{K}}{a_{\theta(\tau)}(\tau)^{2}}}\left(E _ { \tau } ^ { P } \left\{\left[k_{\theta(\tau+1)}(\tau+1)-\right.\right.\right. \\
\left.E_{\tau}^{P}\left[k_{\theta(\tau+1)}(\tau+1) \Delta X_{\theta(\tau)}^{*}(\tau+1)\right] M_{\theta(\tau)}^{-1} k_{\theta(\tau+1)}(\tau+1) \Delta X_{\theta(\tau)}(\tau+1)\right] \\
\left.\left.\frac{H_{\theta(\tau+1)}(\tau+1)}{a_{\theta(\tau)}(\tau)}\right\}\right),
\end{gathered}
$$

$\bar{U}=a_{\theta(\tau)}(\tau)\left[E_{\tau}^{P}\left\{k_{\theta(\tau+1)}(\tau+1) H_{\theta(\tau+1)}(\tau+1)\right\}-\right.$

$$
\begin{array}{r}
E_{\tau}^{P}\left(E_{\tau}^{P}\left(k_{\theta(\tau+1)}(\tau+1) \Delta X_{\theta(\tau)}^{*}(\tau+1)\right) M_{\theta(\tau)}^{-1} k_{\theta(\tau+1)}(\tau+1)\right. \\
\left.\left.H_{\theta(\tau+1)}(\tau+1) \Delta X_{\theta(\tau)}(\tau+1)\right)\right],
\end{array}
$$

$$
\begin{aligned}
& \bar{R}= E_{\tau}^{P}\left\{k_{\theta(\tau+1)}(\tau+1)\left(H_{\theta(\tau+1)}(\tau+1)\right)^{2}\right\}- \\
& E_{\tau}^{P}\left(k_{\theta(\tau+1)}(\tau+1) H_{\theta(\tau+1)}(\tau+1) \Delta X_{\theta(\tau)}^{*}(\tau+1)\right) M_{\theta(\tau)}^{-1} \\
& E_{\tau}^{P}\left(k_{\theta(\tau+1)}(\tau+1) H_{\theta(\tau+1)}(\tau+1) \Delta X_{\theta(\tau)}(\tau+1)\right)+E\left(\delta_{\theta(\tau+1)}\right) .
\end{aligned}
$$


Demonstraçãao. Expandindo o termo quadrático na Expressão (A.27), obtemos:

$$
\begin{gathered}
\frac{\mathcal{J}(V(\tau), \theta(\tau), \tau)-\sum_{j} p_{\theta(\tau), j} E\left(\delta_{j}\right)}{S_{\theta(\tau), 0}(\tau+1)^{2}}=\sum_{j} p_{\theta(\tau), j} E_{\tau}^{P}\left\{k _ { j } ( \tau + 1 ) \left[\left(\frac{V(\tau)}{S_{0}(\tau)}\right)^{2}-\right.\right. \\
\left.\left.2 \frac{V(\tau)}{S_{0}(\tau)} \frac{H_{j}(\tau+1)}{S_{\theta(\tau), 0}(\tau+1)}+\left(\frac{H_{j}(\tau+1)}{S_{\theta(\tau), 0}(\tau+1)}\right)^{2}\right]\right\}- \\
\left(\sum_{j} p_{\theta(\tau), j} E_{\tau}^{P}\left(k_{j}(\tau+1)\left(\frac{V(\tau)}{S_{0}(\tau)}-\frac{H_{j}(\tau+1)}{S_{\theta(\tau), 0}(\tau+1)}\right) \Delta X_{\theta(\tau)}^{*}(\tau+1)\right)\right) \\
M_{\theta(\tau)}^{-1}\left(\sum_{j} p_{\theta(\tau), j} E_{\tau}^{P}\left(k_{j}(\tau+1)\left(\frac{V(\tau)}{S_{0}(\tau)}-\frac{H_{j}(\tau+1)}{S_{\theta(\tau), 0}(\tau+1)}\right) \Delta X_{\theta(\tau)}(\tau+1)\right)\right) .
\end{gathered}
$$

Expandindo os valores esperados, obtemos:

$$
\begin{gathered}
\frac{\mathcal{J}(V(\tau), \theta(\tau), \tau)-\sum_{j} p_{\theta(\tau), j} E\left(\delta_{j}\right)}{S_{\theta(\tau), 0}(\tau+1)^{2}}= \\
\left(\sum_{j} p_{\theta(\tau), j} E_{\tau}^{P}\left\{k_{j}(\tau+1)\left(\frac{V(\tau)}{S_{0}(\tau)}\right)^{2}\right\}\right)- \\
2\left(\sum_{j} p_{\theta(\tau), j} E_{\tau}^{P}\left\{k_{j}(\tau+1) \frac{V(\tau)}{S_{0}(\tau)} \frac{H_{j}(\tau+1)}{S_{\theta(\tau), 0}(\tau+1)}\right\}\right)+ \\
\left(\sum_{j} p_{\theta(\tau), j} E_{\tau}^{P}\left\{k_{j}(\tau+1)\left(\frac{H_{j}(\tau+1)}{S_{\theta(\tau), 0}(\tau+1)}\right)^{2}\right\}\right)- \\
\left(\left(\sum_{j} p_{\theta(\tau), j} E_{\tau}^{P}\left(k_{j}(\tau+1) \frac{V(\tau)}{S_{0}(\tau)} \Delta X_{\theta(\tau)}^{*}(\tau+1)\right)\right)-\right. \\
\left.\left(\sum_{j} p_{\theta(\tau), j} E_{\tau}^{P}\left(k_{j}(\tau+1) \frac{H_{j}(\tau+1)}{S_{\theta(\tau), 0}(\tau+1)} \Delta X_{\theta(\tau)}^{*}(\tau+1)\right)\right)\right) M_{\theta(\tau)}^{-1} \\
\left(\left(\sum_{j} p_{\theta(\tau), j} E_{\tau}^{P}\left(k_{j}(\tau+1) \frac{V(\tau)}{S_{0}(\tau)} \Delta X_{\theta(\tau)}(\tau+1)\right)\right)-\right. \\
\left.\left(\sum_{j} p_{\theta(\tau), j} E_{\tau}^{P}\left(k_{j}(\tau+1) \frac{H_{j}(\tau+1)}{S_{\theta(\tau), 0}(\tau+1)} \Delta X_{\theta(\tau)}(\tau+1)\right)\right)\right)
\end{gathered}
$$


Efetuando as multiplicações cruzadas, obtemos:

$$
\begin{aligned}
& \frac{\mathcal{J}(V(\tau), \theta(\tau), \tau)-\sum_{j} p_{\theta(\tau), j} E\left(\delta_{j}\right)}{S_{\theta(\tau), 0}(\tau+1)^{2}}= \\
& \left(\sum_{j} p_{\theta(\tau), j} E_{\tau}^{P}\left\{k_{j}(\tau+1)\right\}\right) \frac{V(\tau)^{2}}{S_{0}(\tau)^{2}}- \\
& 2\left(\sum_{j} p_{\theta(\tau), j} E_{\tau}^{P}\left\{k_{j}(\tau+1) \frac{H_{j}(\tau+1)}{S_{\theta(\tau), 0}(\tau+1)}\right\}\right) \frac{V(\tau)}{S_{0}(\tau)}+ \\
& \left(\sum_{j} p_{\theta(\tau), j} E_{\tau}^{P}\left\{k_{j}(\tau+1)\left(\frac{H_{j}(\tau+1)}{S_{\theta(\tau), 0}(\tau+1)}\right)^{2}\right\}\right)- \\
& \left\{\left(\sum_{j} p_{\theta(\tau), j} E_{\tau}^{P}\left(k_{j}(\tau+1) \Delta X_{\theta(\tau)}^{*}(\tau+1)\right)\right) M_{\theta(\tau)}^{-1}\right. \\
& \left(\sum_{j} p_{\theta(\tau), j} E_{\tau}^{P}\left(k_{j}(\tau+1) \Delta X_{\theta(\tau)}(\tau+1)\right)\right) \frac{V(\tau)^{2}}{S_{0}(\tau)^{2}}- \\
& \left(\sum_{j} p_{\theta(\tau), j} E_{\tau}^{P}\left(k_{j}(\tau+1) \Delta X_{\theta(\tau)}^{*}(\tau+1)\right)\right) M_{\theta(\tau)}^{-1} \\
& \left(\sum_{j} p_{\theta(\tau), j} E_{\tau}^{P}\left(k_{j}(\tau+1) \frac{H_{j}(\tau+1)}{S_{\theta(\tau), 0}(\tau+1)} \Delta X_{\theta(\tau)}(\tau+1)\right)\right) \frac{V(\tau)}{S_{0}(\tau)}- \\
& \left(\sum_{j} p_{\theta(\tau), j} E_{\tau}^{P}\left(k_{j}(\tau+1) \frac{H_{j}(\tau+1)}{S_{\theta(\tau), 0}(\tau+1)} \Delta X_{\theta(\tau)}^{*}(\tau+1)\right)\right) M_{\theta(\tau)}^{-1} \\
& \left(\sum_{j} p_{\theta(\tau), j} E_{\tau}^{P}\left(k_{j}(\tau+1) \Delta X_{\theta(\tau)}(\tau+1)\right)\right) \frac{V(\tau)}{S_{0}(\tau)}+ \\
& \left(\sum_{j} p_{\theta(\tau), j} E_{\tau}^{P}\left(k_{j}(\tau+1) \frac{H_{j}(\tau+1)}{S_{\theta(\tau), 0}(\tau+1)} \Delta X_{\theta(\tau)}^{*}(\tau+1)\right)\right) M_{\theta(\tau)}^{-1} \\
& \left.\left(\sum_{j} p_{\theta(\tau), j} E_{\tau}^{P}\left(k_{j}(\tau+1) \frac{H_{j}(\tau+1)}{S_{\theta(\tau), 0}(\tau+1)} \Delta X_{\theta(\tau)}(\tau+1)\right)\right)\right\} .
\end{aligned}
$$

Isolando novamente $\mathcal{J}(V(\tau), \theta(\tau), \tau)$ e agrupando os termos em $V(\tau)^{2}$ e 
$V(\tau)$, obtemos:

$$
\begin{gathered}
\mathcal{J}(V(\tau), \theta(\tau), \tau)=\left[\left(\sum_{j} p_{\theta(\tau), j} E_{\tau}^{P}\left\{k_{j}(\tau+1)\right\}\right)-\right. \\
\left(\sum_{j} p_{\theta(\tau), j} E_{\tau}^{P}\left(k_{j}(\tau+1) \Delta X_{\theta(\tau)}^{*}(\tau+1)\right)\right) M_{\theta(\tau)}^{-1} \\
\left.\left(\sum_{j} p_{\theta(\tau), j} E_{\tau}^{P}\left(k_{j}(\tau+1) \Delta X_{\theta(\tau)}(\tau+1)\right)\right)\right] \underbrace{\frac{S_{\theta(\tau), 0}(\tau+1)^{2}}{S_{0}(\tau)^{2}}}_{a_{\theta(\tau)}(\tau)^{2}} V(\tau)^{2}- \\
\left.\left(\sum_{j} p_{\theta(\tau), j} E_{\tau}^{P}\left(k_{j}(\tau+1) \Delta X_{\theta(\tau)}^{*}(\tau+1)\right)\right) M_{\theta(\tau)}^{-1} p_{\theta(\tau), j} E_{\tau}^{P}\left\{k_{j}(\tau+1) H_{j}(\tau+1)\right\}\right)- \\
\left.\left(\sum_{j} p_{\theta(\tau), j} E_{\tau}^{P}\left(k_{j}(\tau+1) H_{j}(\tau+1) \Delta X_{\theta(\tau)}(\tau+1)\right)\right)\right] \underbrace{\frac{\mathrm{S}_{\theta(\tau), 0}(\tau+1)}{\mathrm{S}_{0}(\tau)}}_{a_{\theta(\tau)}(\tau)} V(\tau)+ \\
\left(\sum_{j} p_{\theta(\tau), j} E_{\tau}^{P}\left\{k_{j}(\tau+1)\left(H_{j}(\tau+1)\right)^{2}\right\}\right)- \\
M_{\theta(\tau)}^{-1}\left(\sum_{j} p_{\theta(\tau), j} E_{\tau}^{P}\left(k_{j}(\tau+1) H_{j}(\tau+1) \Delta X_{\theta(\tau)}(\tau+1)\right)\right)+\sum_{j} p_{\theta(\tau), j} E\left(k_{\tau}\left(k_{j}(\tau+1) H_{j}(\tau+1) \Delta X_{\theta(\tau)}^{*}(\tau+1)\right)\right)
\end{gathered}
$$

Fazendo:

$$
\begin{aligned}
\bar{K}=a_{\theta(\tau)}(\tau)^{2} & {\left[\left(\sum_{j} p_{\theta(\tau), j} E_{\tau}^{P}\left\{k_{j}(\tau+1)\right\}\right)-\right.} \\
& \left(\sum_{j} p_{\theta(\tau), j} E_{\tau}^{P}\left(k_{j}(\tau+1) \Delta X_{\theta(\tau)}^{*}(\tau+1)\right)\right) M_{\theta(\tau)}^{-1} \\
& \left.\left(\sum j p_{\theta(\tau), j} E_{\tau}^{P}\left(k_{j}(\tau+1) \Delta X_{\theta(\tau)}(\tau+1)\right)\right)\right],
\end{aligned}
$$




$$
\begin{gathered}
\bar{U}=a_{\theta(\tau)}(\tau)\left[\left(\sum_{j} p_{\theta(\tau), j} E_{\tau}^{P}\left\{k_{j}(\tau+1) H_{j}(\tau+1)\right\}\right)-\right. \\
\left(\sum_{j} p_{\theta(\tau), j} E_{\tau}^{P}\left(k_{j}(\tau+1) \Delta X_{\theta(\tau)}^{*}(\tau+1)\right)\right) M_{\theta(\tau)}^{-1} \\
\left.\left.\left.\left(\sum_{j} p_{\theta(\tau), j} E_{\tau}^{P}\left(k_{j}(\tau+1) H_{j}(\tau+1) \Delta X_{\theta(\tau)}(\tau+1)\right)\right)\right], \quad \text { (A.3.3 }\right)\right] \\
\bar{R}=\left(\sum_{j} p_{\theta(\tau), j} E_{\tau}^{P}\left\{k_{j}(\tau+1)\left(H_{j}(\tau+1)\right)^{2}\right\}\right)- \\
\left(\sum_{j} p_{\theta(\tau), j} E_{\tau}^{P}\left(k_{j}(\tau+1) H_{j}(\tau+1) \Delta X_{\theta(\tau)}^{*}(\tau+1)\right)\right) \\
M_{\theta(\tau)}^{-1}\left(\sum_{j} p_{\theta(\tau), j} E_{\tau}^{P}\left(k_{j}(\tau+1) H_{j}(\tau+1) \Delta X_{\theta(\tau)}(\tau+1)\right)\right)+\sum_{j} p_{\theta(\tau), j} E\left(\delta_{j}\right),
\end{gathered}
$$

podemos escrever a Expressão (A.36) como:

$$
\begin{aligned}
& \mathcal{J}(V(\tau), \theta(\tau), \tau)= \\
& \underbrace{\left(\bar{K}^{1 / 2} V(\tau)\right)^{2}-2 \bar{K}^{1 / 2} \frac{\bar{U}}{\bar{K}^{1 / 2}} V(\tau)+\left(\frac{\bar{U}}{\bar{K}^{1 / 2}}\right)^{2}}_{\left(\bar{K}^{1 / 2} V(\tau)-\frac{\bar{U}}{\bar{K}^{1 / 2}}\right)}+\underbrace{\bar{R}-\left(\frac{\bar{U}}{\bar{K}^{1 / 2}}\right)^{2}}_{\bar{L}} .
\end{aligned}
$$

Portanto:

$$
\mathcal{J}(V(\tau), \theta(\tau), \tau)=\bar{K}\left(V(\tau)-\frac{\bar{U}}{\bar{K}}\right)^{2}+\underbrace{\bar{R}-\frac{\bar{U}^{2}}{\bar{K}}}_{\bar{L}} .
$$

O termo $\bar{K}$ pode ser rescrito deixando-se implícito o valor esperado em relação à VA $\theta(\tau+1)$ (que está explícito na Expressão (A.37) devido à presença $\left.\operatorname{dos} p_{\theta(\tau), j}\right)$. Assim:

$$
\begin{aligned}
& \bar{K}=a_{\theta(\tau)}(\tau)^{2}\left[E_{\tau}^{P}\left\{k_{\theta(\tau+1)}(\tau+1)\right\}-\right. \\
& \left.E_{\tau}^{P}\left(k_{\theta(\tau+1)}(\tau+1) \Delta X_{\theta(\tau)}^{*}(\tau+1)\right) M_{\theta(\tau)}^{-1} E_{\tau}^{P}\left(k_{\theta(\tau+1)}(\tau+1) \Delta X_{\theta(\tau)}(\tau+1)\right)\right],
\end{aligned}
$$

onde $M_{\theta(\tau)}$, dado pela Expressão (A.43), pode ser rescrito como:

$$
M_{\theta(\tau)}=E_{\tau}^{P}\left\{k_{\theta(\tau+1)}(\tau+1) \Delta X_{\theta(\tau)}(\tau+1) \Delta X_{\theta(\tau)}^{*}(\tau+1)\right\} .
$$


O termo $\bar{U}$ (Equação (A.38)) também pode ser rescrito. Escrevendo o valor esperado com relação à VA $\theta(\tau+1)$ de forma implícita, obtemos:

$$
\begin{aligned}
\bar{U}=a_{\theta(\tau)}(\tau)\left[E_{\tau}^{P}\left\{k_{\theta(\tau+1)}(\tau+1) H_{\theta(\tau+1)}(\tau+1)\right\}-\right. \\
E_{\tau}^{P}\left(k_{\theta(\tau+1)}(\tau+1) \Delta X_{\theta(\tau)}^{*}(\tau+1)\right) M_{\theta(\tau)}^{-1} \\
\left.E_{\tau}^{P}\left(k_{\theta(\tau+1)}(\tau+1) H_{\theta(\tau+1)}(\tau+1) \Delta X_{\theta(\tau)}(\tau+1)\right)\right],
\end{aligned}
$$

O segundo termo da Expressão (A.44) pode ser rescrito "jogando-se"o termo $E_{\tau}^{P}\left(k_{\theta(\tau+1)}(\tau+1) \Delta X_{\theta(\tau)}^{*}(\tau+1)\right) M_{\theta(\tau)}^{-1}$, que é é $\mathcal{F}_{\tau}$-mensurável, para dentro do valor esperado:

$$
\begin{gathered}
\bar{U}=a_{\theta(\tau)}(\tau)\left[E_{\tau}^{P}\left\{k_{\theta(\tau+1)}(\tau+1) H_{\theta(\tau+1)}(\tau+1)\right\}-\right. \\
E_{\tau}^{P}\left(E_{\tau}^{P}\left(k_{\theta(\tau+1)}(\tau+1) \Delta X_{\theta(\tau)}^{*}(\tau+1)\right) M_{\theta(\tau)}^{-1}\right. \\
\left.\left.k_{\theta(\tau+1)}(\tau+1) H_{\theta(\tau+1)}(\tau+1) \Delta X_{\theta(\tau)}(\tau+1)\right)\right] .
\end{gathered}
$$

Unificando os valores esperados e simplificando, obtemos:

$$
\begin{gathered}
\bar{U}=a_{\theta(\tau)}(\tau)^{2} E_{\tau}^{P}\left\{\left[k_{\theta(\tau+1)}(\tau+1)-\right.\right. \\
\left.E_{\tau}^{P}\left[k_{\theta(\tau+1)}(\tau+1) \Delta X_{\theta(\tau)}^{*}(\tau+1)\right] M_{\theta(\tau)}^{-1} k_{\theta(\tau+1)}(\tau+1) \Delta X_{\theta(\tau)}(\tau+1)\right] \\
\left.\frac{H_{\theta(\tau+1)}(\tau+1)}{a_{\theta(\tau)}(\tau)}\right\} \cdot
\end{gathered}
$$

O termo $\bar{H}=\frac{\bar{U}}{\bar{K}}$ da Expressão (A.49) pode ser então escrito como:

$$
\begin{aligned}
\bar{H}=\frac{\bar{U}}{\bar{K}}= & \frac{1}{\frac{\bar{K}}{a_{\theta(\tau)}(\tau)^{2}}}\left(E _ { \tau } ^ { P } \left\{\left[k_{\theta(\tau+1)}(\tau+1)-E_{\tau}^{P}\left[k_{\theta(\tau+1)}(\tau+1) \Delta X_{\theta(\tau)}^{*}(\tau+1)\right]\right.\right.\right. \\
& \left.\left.\left.\quad M_{\theta(\tau)}^{-1} k_{\theta(\tau+1)}(\tau+1) \Delta X_{\theta(\tau)}(\tau+1)\right] \frac{H_{\theta(\tau+1)}(\tau+1)}{a_{\theta(\tau)}(\tau)}\right\}\right) .
\end{aligned}
$$

De forma análoga, o termo $\bar{R}$ (Equação (A.39)) pode ser rescrito como:

$$
\begin{aligned}
\bar{R}= & E_{\tau}^{P}\left\{k_{\theta(\tau+1)}(\tau+1)\left(H_{\theta(\tau+1)}(\tau+1)\right)^{2}\right\}- \\
& E_{\tau}^{P}\left(k_{\theta(\tau+1)}(\tau+1) H_{\theta(\tau+1)}(\tau+1) \Delta X_{\theta(\tau)}^{*}(\tau+1)\right) M_{\theta(\tau)}^{-1} \\
& E_{\tau}^{P}\left(k_{\theta(\tau+1)}(\tau+1) H_{\theta(\tau+1)}(\tau+1) \Delta X_{\theta(\tau)}(\tau+1)\right)+E\left(\delta_{\theta(\tau+1)}\right) .
\end{aligned}
$$

Finalmente, a Expressão (A.49) pode ser rescrita como:

$$
\mathcal{J}(V(\tau), \theta(\tau), \tau)=\bar{K}(V(\tau)-\bar{H})^{2}+\underbrace{\bar{R}-\frac{\bar{U}^{2}}{\bar{K}}}_{\bar{L}},
$$


onde $\bar{K}$ é dado pela Equação (A.66), $\bar{H}$ pela Equação (A.47), $\bar{R}$ pela Equação (A.48) e $\bar{U}$ pela Equação (A.46).

Demonstração do Teorema 3.1. A prova é feita por indução finita. Para $\tau=\mathcal{T}$ o resultado é claramente verdadeiro, pois:

$$
\overline{\mathcal{J}}(V(\mathcal{T}), \theta(\mathcal{T}), \mathcal{T})=\left(V(\mathcal{T})-H_{\theta(\mathcal{T})}(\mathcal{T})\right)^{2},
$$

já que:

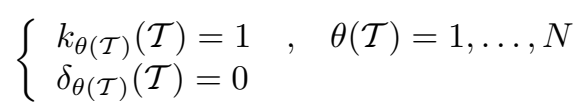

Suponhamos que o resultado valha para $\tau+1$. Pela equação de Bellman, temos que:

$$
\begin{gathered}
\overline{\mathcal{J}}(V(\tau), \theta(\tau), \tau)= \\
\min _{u(\tau)} E^{P}\left\{\overline{\mathcal{J}}(V(\tau+1), \theta(\tau+1), \tau+1) \mid \mathcal{F}_{\tau}\right\}= \\
\min _{u(\tau)} E^{P}\left\{k_{\theta(\tau+1)}(\tau+1)\left(V(\tau+1)-H_{\theta(\tau+1)}(\tau+1)\right)^{2}+\delta_{\theta(\tau+1)}(\tau+1) \mid \mathcal{F}_{\tau}\right\}= \\
\left(\min _{u(\tau)} E^{P}\left\{k_{\theta(\tau+1)}(\tau+1)\left(V(\tau+1)-H_{\theta(\tau+1)}(\tau+1)\right)^{2} \mid \mathcal{F}_{\tau}\right\}\right)+ \\
E^{P}\left\{\delta_{\theta(\tau+1)}(\tau+1) \mid \mathcal{F}_{\tau}\right\} \quad(\mathrm{A} .52)
\end{gathered}
$$

onde:

$$
E^{P}\left(\delta_{\theta(\tau+1)}(\tau+1) \mid \mathcal{F}_{\tau}\right)=\sum_{j} p_{\theta(\tau+1), j} E^{P}\left(\delta_{j}(\tau+1) \mid \mathcal{F}_{\tau}\right),
$$

e

$$
\begin{aligned}
& E^{P}\left(k_{\theta(\tau+1)}(\tau+1)\left(V(\tau+1)-H_{\theta(\tau+1)}(\tau+1)\right)^{2} \mid \mathcal{F}_{\tau}\right)= \\
& E^{P}\left[k_{\theta(\tau+1)}(\tau+1)\right. \\
& \left.(\underbrace{a_{\theta(\tau)}(\tau) V(\tau)+S_{\theta(\tau), 0}(\tau+1) \Delta X_{\theta(\tau)}^{*}(\tau+1) u(\tau)}_{V(\tau+1)}-H_{\theta(\tau+1)}(\tau+1))^{2} \mid \mathcal{F}_{\tau}\right]= \\
& E^{P}\left[k_{\theta(\tau+1)}(\tau+1) S_{\theta(\tau), 0}(\tau+1)^{2}\right. \\
& \left.\left(\frac{a_{\theta(\tau)}(\tau)}{S_{\theta(\tau), 0}(\tau+1)} V(\tau)+\Delta X_{\theta(\tau)}^{*}(\tau+1) u(\tau)-\frac{H_{\theta(\tau+1)}(\tau+1)}{S_{\theta(\tau), 0}(\tau+1)}\right)^{2} \mid \mathcal{F}_{\tau}\right]
\end{aligned}
$$

Lembrando que $S_{\theta(\tau), 0}(\tau+1)=S_{0}(\tau) a_{\theta(\tau)}(\tau)$, a expressão acima pode ser 
rescrita como:

$$
\begin{gathered}
E^{P}\left(k_{\theta(\tau+1)}(\tau+1)\left(V(\tau+1)-H_{\theta(\tau+1)}(\tau+1)\right)^{2} \mid \mathcal{F}_{\tau}\right)= \\
E^{P}\left(k_{\theta(\tau+1)}(\tau+1) S_{\theta(\tau), 0}(\tau+1)^{2}\right. \\
\left.\left(\frac{V(\tau)}{S_{0}(\tau)}+\Delta X_{\theta(\tau)}^{*}(\tau+1) u(\tau)-\frac{H_{\theta(\tau+1)}(\tau+1)}{S_{\theta(\tau), 0}(\tau+1)}\right)^{2} \mid \mathcal{F}_{\tau}\right)
\end{gathered}
$$

Calculando explicitamente o valor esperado em relação à VA $\theta(\tau+1)$, obtemos:

$$
\begin{gathered}
E^{P}\left(k_{\theta(\tau+1)}(\tau+1)\left(V(\tau+1)-H_{\theta(\tau+1)}(\tau+1)\right)^{2} \mid \mathcal{F}_{\tau}\right)= \\
\sum_{j} p_{\theta(\tau), j} E^{P}\left\{k_{j}(\tau+1) S_{\theta(\tau), 0}(\tau+1)^{2}\right. \\
\left.\left(\frac{V(\tau)}{S_{0}(\tau)}+\Delta X_{\theta(\tau)}^{*}(\tau+1) u(\tau)-\frac{H_{j}(\tau+1)}{S_{\theta(\tau), 0}(\tau+1)}\right)^{2} \mid \mathcal{F}_{\tau}\right\} .
\end{gathered}
$$

Escrevendo:

$$
X_{j}=S_{\theta(\tau), 0}(\tau+1) \sqrt{k_{j}(\tau+1)} \Delta X_{\theta(\tau)}(\tau+1)
$$

$$
Y_{j}=S_{\theta(\tau), 0}(\tau+1) \sqrt{k_{j}(\tau+1)}\left(\frac{V(\tau)}{S_{0}(\tau)}-\frac{H_{j}(\tau+1)}{S_{\theta(\tau), 0}(\tau+1)}\right),
$$

o problema apresentado em (A.52) pode ser rescrito da seguinte forma:

$$
\left(\min _{u(\tau)} \sum_{j} p_{\theta(\tau), j} E\left\{\left(Y_{j}+X_{j}^{*} u(\tau)\right)^{2} \mid \mathcal{F}_{\tau}\right\}\right)+\sum_{j} p_{\theta(\tau), j} E^{P}\left(\delta_{j}(\tau+1) \mid \mathcal{F}_{\tau}\right) .
$$


Conforme o Lema A.1, a solução ótima $\bar{u}(\tau)$ desse problema é:

$$
\begin{aligned}
& \bar{u}(\tau)=-\left[\sum_{j} p_{\theta(\tau), j} E_{\tau}^{P}\left(X_{j} X_{j}^{*}\right)\right]^{-1}\left(\sum_{j} p_{\theta(\tau), j} E_{\tau}^{P}\left(X_{j} Y_{j}\right)\right)= \\
& -\left[\sum_{j} p_{\theta(\tau), j} E_{\tau}^{P}\{\underbrace{S_{\theta(\tau), 0}(\tau+1) \sqrt{k_{j}(\tau+1)} \Delta X_{\theta(\tau)}(\tau+1)}_{X_{j}}\right. \\
& \underbrace{S_{\theta(\tau), 0}(\tau+1) \sqrt{k_{j}(\tau+1)} \Delta X_{\theta(\tau)}^{*}(\tau+1)}_{X_{j}^{*}}\}]^{-1} \\
& \sum_{j} p_{\theta(\tau), j} E_{\tau}^{P}\{\underbrace{S_{\theta(\tau), 0}(\tau+1) \sqrt{k_{j}(\tau+1)} \Delta X_{\theta(\tau)}(\tau+1)}_{X_{j}} \\
& \underbrace{S_{\theta(\tau), 0}(\tau+1) \sqrt{k_{j}(\tau+1)}\left(\frac{V(\tau)}{S_{0}(\tau)}-\frac{H_{j}(\tau+1)}{S_{\theta(\tau), 0}(\tau+1)}\right)}_{Y_{j}}\} .
\end{aligned}
$$

Como $S_{\theta(\tau), 0}(\tau+1)$ é um processo previsível e, portanto, $\mathcal{F}_{\tau}$-mensurável, podemos simplificar esta expressão, obtemos:

$$
\begin{gathered}
\bar{u}(\tau)=-\left[\sum_{j} p_{\theta(\tau), j} E_{\tau}^{P}\left\{k_{j}(\tau+1) \Delta X_{\theta(\tau)}(\tau+1) \Delta X_{\theta(\tau)}^{*}(\tau+1)\right\}\right]^{-1} \\
\sum_{j} p_{\theta(\tau), j} E_{\tau}^{P}\left\{k_{j}(\tau+1) \Delta X_{\theta(\tau)}(\tau+1)\left(\frac{V(\tau)}{S_{0}(\tau)}-\frac{H_{j}(\tau+1)}{S_{\theta(\tau), 0}(\tau+1)}\right)\right\}= \\
-\left[E_{\tau}^{P}\left\{k_{\theta(\tau+1)}(\tau+1) \Delta X_{\theta(\tau)}(\tau+1) \Delta X_{\theta(\tau)}^{*}(\tau+1)\right\}\right]^{-1} \\
E_{\tau}^{P}\left\{k_{\theta(\tau+1)}(\tau+1) \Delta X_{\theta(\tau)}(\tau+1)\left(\frac{V(\tau)}{S_{0}(\tau)}-\frac{H_{\theta(\tau+1)}(\tau+1)}{S_{\theta(\tau), 0}(\tau+1)}\right)\right\} .
\end{gathered}
$$

Usando novamente os resultados do Lema A.1, podemos obter a função ob- 
jetivo $\overline{\mathcal{J}}(V(\tau), \theta(\tau), \tau)$ correspondente a $\bar{u}(\tau)$ :

$$
\begin{gathered}
\overline{\mathcal{J}}(V(\tau), \theta(\tau), \tau)=\sum_{j} p_{\theta(\tau), j} E_{\tau}^{P}\left(Y_{j}^{2}\right)- \\
\left(\sum_{j} p_{\theta(\tau), j} E_{\tau}^{P}\left(Y_{j} X_{j}^{*}\right)\right)\left[\sum_{j} p_{\theta(\tau), j} E_{\tau}^{P}\left(X_{j} X_{j}^{*}\right)\right]^{-1} \\
\left(\sum_{j} p_{\theta(\tau), j} E_{\tau}^{P}\left(X_{j} Y_{j}\right)\right)+\sum_{j} p_{\theta(\tau), j} E_{\tau}^{P}\left(\delta_{j}\right)= \\
\sum_{j} p_{\theta(\tau), j} E_{\tau}^{P}\left\{\left[S_{\theta(\tau), 0}(\tau+1) \sqrt{k_{j}(\tau+1)}\left(\frac{V(\tau)}{S_{0}(\tau)}-\frac{H_{j}(\tau+1)}{S_{\theta(\tau), 0}(\tau+1)}\right)\right]^{2}\right\}- \\
\sum_{j} p_{\theta(\tau), j} E_{\tau}^{P}\left\{S_{\theta(\tau), 0}(\tau+1) \sqrt{k_{j}(\tau+1)} \Delta X_{\theta(\tau)}(\tau+1)\right. \\
\left.\left.S_{\theta(\tau), 0}(\tau+1) \sqrt{k_{j}(\tau+1)} \Delta X_{\theta(\tau)}^{*}(\tau+1)\right\}\right]^{-1} \\
\left(\sum _ { j } p _ { \theta ( \tau ) , j } E _ { \tau } ^ { P } \left\{S_{\theta(\tau), 0}(\tau+1) \sqrt{k_{j}(\tau+1)} \Delta X_{\theta(\tau)}(\tau+1)\right.\right. \\
\left.\left.S_{\theta(\tau), 0}(\tau+1) \sqrt{k_{j}(\tau+1)}\left(\frac{V(\tau)}{S_{0}(\tau)}-\frac{H_{j}(\tau+1)}{S_{\theta(\tau), 0}(\tau+1)}\right)\right\}\right)+
\end{gathered}
$$

Simplificando esta expressão, obtemos:

$$
\begin{gathered}
\overline{\mathcal{J}}(V(\tau), \theta(\tau), \tau)=S_{\theta(\tau), 0}(\tau+1)^{2} \\
\left\{\sum_{j} p_{\theta(\tau), j} E_{\tau}^{P}\left\{k_{j}(\tau+1)\left(\frac{V(\tau)}{S_{0}(\tau)}-\frac{H_{j}(\tau+1)}{S_{\theta(\tau), 0}(\tau+1)}\right)^{2}\right\}-\right. \\
\left(\sum_{j} p_{\theta(\tau), j} E_{\tau}^{P}\left(k_{j}(\tau+1)\left(\frac{V(\tau)}{S_{0}(\tau)}-\frac{H_{j}(\tau+1)}{S_{\theta(\tau), 0}(\tau+1)}\right) \Delta X_{\theta(\tau)}^{*}(\tau+1)\right)\right) M_{\theta(\tau)}^{-1} \\
\left.\left(\sum_{j} p_{\theta(\tau), j} E_{\tau}^{P}\left(k_{j}(\tau+1)\left(\frac{V(\tau)}{S_{0}(\tau)}-\frac{H_{j}(\tau+1)}{S_{\theta(\tau), 0}(\tau+1)}\right) \Delta X_{\theta(\tau)}(\tau+1)\right)\right)\right\} \\
+\sum_{j} p_{\theta(\tau), j} E\left(\delta_{j}\right),
\end{gathered}
$$

onde:

$$
\begin{array}{r}
M_{\theta(\tau)}=\sum_{j} p_{\theta(\tau), j} E_{\tau}^{P}\left\{k_{j}(\tau+1) \Delta X_{\theta(\tau)}(\tau+1) \Delta X_{\theta(\tau)}^{*}(\tau+1)\right\}= \\
E_{\tau}^{P}\left\{k_{\theta(\tau+1)}(\tau+1) \Delta X_{\theta(\tau)}(\tau+1) \Delta X_{\theta(\tau)}^{*}(\tau+1)\right\},
\end{array}
$$


Para completar a prova por indução finita, precisamos mostrar que $\overline{\mathcal{J}}(V(\tau), \theta(\tau), \tau)$ pode ser escrito da seguinte forma:

$$
\overline{\mathcal{J}}(V(\tau), \theta(\tau), \tau)=k_{\theta(\tau)}(\tau)\left(V(\tau)-H_{\theta(\tau)}(\tau)\right)^{2}+\delta_{\theta(\tau)}(\tau)
$$

Isso, de fato, pode ser feito. De acordo com o Lema A.3, a Expressão (A.63) pode ser expressa na forma da Expressão (A.65) se assumirmos que:

$$
\begin{aligned}
& k_{\theta(\tau)}(\tau)=a_{\theta(\tau)}(\tau)^{2}\left[E_{\tau}^{P}\left\{k_{\theta(\tau+1)}(\tau+1)\right\}-\right. \\
& \left.E_{\tau}^{P}\left(k_{\theta(\tau+1)}(\tau+1) \Delta X_{\theta(\tau)}^{*}(\tau+1)\right) M_{\theta(\tau)}^{-1} E_{\tau}^{P}\left(k_{\theta(\tau+1)}(\tau+1) \Delta X_{\theta(\tau)}(\tau+1)\right)\right]
\end{aligned}
$$

$$
\begin{gathered}
H_{\theta(\tau)}(\tau)=\frac{1}{\frac{k_{\theta(\tau)}(\tau)}{a_{\theta(\tau)}(\tau)^{2}}} \\
\left(E _ { \tau } ^ { P } \left\{\left[k_{\theta(\tau+1)}(\tau+1)-E_{\tau}^{P}\left[k_{\theta(\tau+1)}(\tau+1) \Delta X_{\theta(\tau)}^{*}(\tau+1)\right]\right.\right.\right. \\
\left.\left.\left.M_{\theta(\tau)}^{-1} k_{\theta(\tau+1)}(\tau+1) \Delta X_{\theta(\tau)}(\tau+1)\right] \frac{H_{\theta(\tau+1)}(\tau+1)}{a_{\theta(\tau)}(\tau)}\right\}\right) \\
M_{\theta(\tau)}=E_{\tau}^{P}\left\{k_{\theta(\tau+1)}(\tau+1) \Delta X_{\theta(\tau)}(\tau+1) \Delta X_{\theta(\tau)}^{*}(\tau+1)\right\}
\end{gathered}
$$

$$
\delta_{\theta(\tau)}(\tau)=\bar{L}=\bar{R}-\frac{\bar{U}^{2}}{\bar{K}},
$$

conforme definido no Lema A.3.

Para encontrarmos uma expressão mais simples para $\delta_{\theta(\tau)}(\tau)$, resolvamos o problema dado pela Equação (A.59) considerando:

$$
X_{j}=S_{\theta(\tau), 0}(\tau+1) \sqrt{k_{j}(\tau+1)} \Delta X_{\theta(\tau)}(\tau+1)
$$

$\mathrm{e}$

$$
Y_{j}=S_{\theta(\tau), 0}(\tau+1) \sqrt{k_{j}(\tau+1)}\left(\frac{H_{\theta(\tau)}(\tau)}{S_{0}(\tau)}-\frac{H_{j}(\tau+1)}{S_{\theta(\tau), 0}(\tau+1)}\right) .
$$


Assim, o problema dado pela Equação (A.59) se transforma em:

$$
\begin{gathered}
\left(\min _{u(\tau)} \sum_{j} p_{\theta(\tau), j} E\left\{\left(Y_{j}+X_{j}^{*} u(\tau)\right)^{2} \mid \mathcal{F}_{\tau}\right\}\right)+\sum_{j} p_{\theta(\tau), j} E\left(\delta_{j}(\tau+1) \mid \mathcal{F}_{\tau}\right)= \\
\left(\operatorname { m i n } _ { u ( \tau ) } \sum _ { j } p _ { \theta ( \tau ) , j } E \left\{k _ { j } ( \tau + 1 ) \left(a_{\theta(\tau)}(\tau) H_{\theta(\tau)}(\tau)+\right.\right.\right. \\
\left.\left.\left.S_{\theta(\tau), 0}(\tau+1) \Delta X_{\theta(\tau)}^{*}(\tau+1) u(\tau)-H_{j}(\tau+1)\right)^{2} \mid \mathcal{F}_{\tau}\right\}\right)+ \\
\sum_{j} p_{\theta(\tau), j} E\left(\delta_{j}(\tau+1) \mid \mathcal{F}_{\tau}\right)= \\
\left(\operatorname { m i n } _ { u ( \tau ) } E \left\{k _ { \theta ( \tau + 1 ) } ( \tau + 1 ) \left(a_{\theta(\tau)}(\tau) H_{\theta(\tau)}(\tau)+\right.\right.\right. \\
\left.\left.\left.S_{\theta(\tau), 0}(\tau+1) \Delta X_{\theta(\tau)}^{*}(\tau+1) u(\tau)-H_{\theta(\tau+1)}(\tau+1)\right)^{2} \mid \mathcal{F}_{\tau}\right\}\right)+ \\
E\left(\delta_{\theta(\tau+1)}(\tau+1) \mid \mathcal{F}_{\tau}\right) . \quad(\mathrm{A} .72)
\end{gathered}
$$

Conforme o Lema A.1, a solução ótima $u(\tau)$ desse problema é:

$$
\begin{gathered}
u(\tau)=-\left[\sum_{j} p_{\theta(\tau), j} E_{\tau}^{P}\left\{k_{j}(\tau+1) \Delta X_{\theta(\tau)}(\tau+1) \Delta X_{\theta(\tau)}^{*}(\tau+1)\right\}\right]^{-1} \\
\sum_{j} p_{\theta(\tau), j} E_{\tau}^{P}\left\{k_{j}(\tau+1) \Delta X_{\theta(\tau)}(\tau+1)\left(\frac{H_{\theta(\tau)}(\tau)}{S_{0}(\tau)}-\frac{H_{j}(\tau+1)}{S_{\theta(\tau), 0}(\tau+1)}\right)\right\}= \\
-\left[E_{\tau}^{P}\left\{k_{\theta(\tau+1)}(\tau+1) \Delta X_{\theta(\tau)}(\tau+1) \Delta X_{\theta(\tau)}^{*}(\tau+1)\right\}\right]^{-1} \\
E_{\tau}^{P}\left\{k_{\theta(\tau+1)}(\tau+1) \Delta X_{\theta(\tau)}(\tau+1)\left(\frac{H_{\theta(\tau)}(\tau)}{S_{0}(\tau)}-\frac{H_{\theta(\tau+1)}(\tau+1)}{S_{\theta(\tau), 0}(\tau+1)}\right)\right\} .
\end{gathered}
$$

De forma análoga à que procedemos anteriormente, podemos usar novamente os Lemas A.1 e A.3 para obter a função objetivo $\overline{\mathcal{J}}\left(H_{\theta(\tau)}(\tau), \theta(\tau), \tau\right)$ correspondente a $u(\tau)$ :

$$
\overline{\mathcal{J}}\left(H_{\theta(\tau)}(\tau), \theta(\tau), \tau\right)=k_{\theta(\tau)}(\tau)\left(H_{\theta(\tau)}(\tau)-H_{\theta(\tau)}(\tau)\right)^{2}+\delta_{\theta(\tau)}(\tau)=\delta_{\theta(\tau)}(\tau) .
$$

Portanto, a função objetivo é precisamente $\delta_{\theta(\tau)}(\tau)$, que queríamos simplifi- 
car. Assim:

$$
\begin{gathered}
\delta_{\theta(\tau)}(\tau)=\left(\sum _ { j } p _ { \theta ( \tau ) , j } E _ { \tau } ^ { P } \left\{k _ { j } ( \tau + 1 ) \left(a_{\theta(\tau)}(\tau) H_{\theta(\tau)}(\tau)+\right.\right.\right. \\
\left.\left.\left.S_{\theta(\tau), 0}(\tau+1) \Delta X_{\theta(\tau)}^{*}(\tau+1) u(\tau)-H_{j}(\tau+1)\right)^{2}\right\}\right)+ \\
\sum_{j} p_{\theta(\tau), j} E_{\tau}^{P}\left(\delta_{j}(\tau+1)\right)= \\
E_{\tau}^{P}\left\{k _ { \theta ( \tau + 1 ) } ( \tau + 1 ) \left(a_{\theta(\tau)}(\tau) H_{\theta(\tau)}(\tau)+S_{\theta(\tau), 0}(\tau+1) \Delta X_{\theta(\tau)}^{*}(\tau+1) u(\tau)-\right.\right. \\
\left.\left.H_{\theta(\tau+1)}(\tau+1)\right)^{2}\right\}+E_{\tau}^{P}\left(\delta_{\theta(\tau+1)}(\tau+1)\right),
\end{gathered}
$$

onde $u(\tau)$ é dado pela Equação (A.73).

De acordo com o Lema A.2, $k_{\theta(\tau+1)}(\tau+1)>0$. Além disso:

$$
\begin{array}{r}
E_{\tau}^{P}\left\{k _ { \theta ( \tau + 1 ) } ( \tau + 1 ) \left(a_{\theta(\tau)}(\tau) H_{\theta(\tau)}(\tau)+S_{\theta(\tau), 0}(\tau+1) \Delta X_{\theta(\tau)}^{*}(\tau+1) u(\tau)-\right.\right. \\
\left.\left.H_{\theta(\tau+1)}(\tau+1)\right)^{2}\right\} \geq 0, \quad(\mathrm{~A} .
\end{array}
$$

por se tratar de uma variável positiva multiplicada por uma expressão quadrática, que, evidentemente, é não-negativa. Como $\delta_{\theta(\mathcal{T})}(\mathcal{T})=0$, é fácil ver (e provar por indução finita) que $\delta_{\theta(\tau)}(\tau) \geq 0, \tau=\mathcal{T}, \ldots, 0$.

Demonstração do Teorema 3.2. Derivando $\overline{\mathcal{J}}(V(0), \theta(0), 0)$ em relação a $V(0)$ e igualando a zero, obtemos:

$$
\begin{aligned}
& {\left[\frac{\partial}{\partial(V(0))}(\overline{\mathcal{J}}(V(0), \theta(0), 0))\right]_{V(0)=\bar{V}(0)}=} \\
& \quad\left[\frac{\partial}{\partial(V(0))}\left(k_{\theta(0)}(0)\left(V(0)-H_{\theta(0)}(0)\right)^{2}+\delta_{\theta(0)}(0)\right)\right]_{V(0)=\bar{V}(0)}=0 .
\end{aligned}
$$

Portanto:

$$
2 k_{\theta(\tau)}(0)\left(\bar{V}(0)-H_{\theta(0)}(0)\right)=0 \Longrightarrow \bar{V}(0)=H_{\theta(0)}(0) .
$$

Demonstração do Teorema 3.3. O erro de hedging esperado quadrático em $\tau=0$ é dado pela Equação (3.16). Do Teorema 3.2, $\bar{V}(0)=H_{\theta(0)}(0)$. Portanto:

$$
\overline{\mathcal{J}}_{0, \mathcal{T}}(\theta(0), 0)=\overline{\mathcal{J}}(\bar{V}(0), \theta(0), 0)=\overline{\mathcal{J}}\left(H_{\theta(0)}(0), \theta(0), 0\right),
$$

Usando a Equação (3.19) para calcular a expressão acima, obtemos que:

$$
\overline{\mathcal{J}}\left(H_{\theta(0)}(0), \theta(0), 0\right)=k_{\theta(0)}(0)\left(H_{\theta(0)}(0)-H_{\theta(0)}(0)\right)^{2}+\delta_{\theta(0)}(0)=\delta_{\theta(0)}(0)
$$

Portanto:

$$
\overline{\mathcal{J}}_{0, \mathcal{T}}(\theta(0), 0)=\delta_{\theta(0)}(0),
$$

i.e., o erro de hedging esperado quadrático em $\tau=0$ é dado por $\delta_{\theta(0)}(0)$. 
Demonstração do Teorema 3.4. A prova é feita por indução finita.

Inicialmente, notemos que esta expressão se verifica para $\tau=\mathcal{T}-1$. De fato:

$$
\begin{array}{r}
H(\mathcal{T}-1)=E_{\mathcal{T}-1}^{P}\left\{\left(\prod_{j=\mathcal{T}-1}^{\mathcal{T}-1} m_{j+1, j}^{P \rightarrow Q}(\theta(j+1))\right) \frac{H(\mathcal{T})}{\prod_{j=\mathcal{T}-1}^{\mathcal{T}-1} a_{\theta(j)}(j)}\right\}= \\
E_{\mathcal{T}-1}^{P}\left\{m_{\mathcal{T}, \mathcal{T}-1}^{P \rightarrow Q} \frac{H(\mathcal{T})}{a_{\theta(\mathcal{T}-1)}(\mathcal{T}-1)}\right\} .
\end{array}
$$

Para o instante $\tau-1$, a Expressão (3.42) se torna:

$$
H_{\theta(\tau-1)}(\tau-1)=E_{\tau-1}^{P}\left\{m_{\tau, \tau-1}^{P \rightarrow Q}(\theta(\tau)) \frac{H_{\theta(\tau)}(\tau)}{a_{\theta(\tau-1)}(\tau-1)}\right\} .
$$

Assumindo que seja verdadeira a Expressão (3.43), e substituindo esta expressão (juntamente com a Expressão (3.44)) na Equação (A.83), obtemos:

$$
\begin{aligned}
& H_{\theta(\tau-1)}(\tau-1)= \\
& E_{\tau-1}^{P}\left\{m_{\tau, \tau-1}^{P \rightarrow Q}(\theta(\tau)) \frac{E_{\tau}^{P}\left\{\prod_{j=\tau}^{\mathcal{T}-1} m_{j+1, j}^{P \rightarrow Q}(\theta(j+1)) \frac{H_{\theta(\mathcal{T})}(\mathcal{T})}{\prod_{j=\tau}^{\mathcal{T}-1} a_{\theta(j)}(j)}\right\}}{a_{\theta(\tau-1)}(\tau-1)}\right\}= \\
& E_{\tau-1}^{P}\left\{E_{\tau}^{P}\left\{\prod_{j=\tau-1}^{\mathcal{T}-1} m_{j+1, j}^{P \rightarrow Q}(\theta(j+1)) \frac{H_{\theta(\mathcal{T})}(\mathcal{T})}{\prod_{j=\tau-1}^{\mathcal{T}-1} a_{\theta(j)}(j)}\right\}\right\}
\end{aligned}
$$

Usando a lei das expectativas iteradas, i.e., $E_{\tau-1}^{P}\left\{E_{\tau}^{P}\{X\}\right\}=E_{\tau-1}^{P}\{X\}$, a última expressão pode ser simplificada para:

$$
H_{\theta(\tau-1)}(\tau-1)=E_{\tau-1}^{P}\left\{\prod_{j=\tau-1}^{\mathcal{T}-1} m_{j+1, j}^{P \rightarrow Q}(\theta(j+1)) \frac{H_{\theta(\mathcal{T})}(\mathcal{T})}{\prod_{j=\tau-1}^{\mathcal{T}-1} a_{\theta(j)}(j)}\right\}
$$

Usando o fato de que $m_{j+1, j}^{P \rightarrow Q}$ e $m_{j, j-1}^{P \rightarrow Q}$ são independentes, e que $E_{\tau}^{P}\left\{m_{\tau+1, \tau}^{P \rightarrow Q}(\theta(\tau+1))\right\}=$ 1, podemos escrever:

$$
\begin{aligned}
E_{\tau-1}^{P}\left\{m_{\mathcal{T}, \tau}^{P \rightarrow Q}\right\}=E_{\tau}^{P}\left\{\prod_{j=\tau-1}^{\mathcal{T}-1} m_{j+1, j}^{P \rightarrow Q}(\theta(j+1))\right\}= \\
\prod_{j=\tau-1}^{\mathcal{T}-1} E_{\tau}^{P}\left\{m_{j+1, j}^{P \rightarrow Q}(\theta(j+1))\right\}=1 .
\end{aligned}
$$

Além disso, $m_{\mathcal{T}, \tau}^{P \rightarrow Q}$ é $\mathcal{F}_{\mathcal{T}}$-mensurável. Portanto, a expressão $m_{\mathcal{T}, \tau}^{P \rightarrow Q}$ pode ser interpretada como uma mudança de medida de probabilidade da medida $P$ para 
a medida $Q$ (i.e., a derivada de Radon-Nikodým, $\frac{\mathrm{dQ}}{\mathrm{dP}}$,da medida $Q$ com relação à medida $P$ ), e a Expressão (A.85) pode ser rescrita simplesmente como:

$$
H_{\theta(\tau-1)}(\tau-1)=E_{\tau-1}^{Q}\left\{\frac{H_{\theta(\mathcal{T})}(\mathcal{T})}{\prod_{j=\tau-1}^{\mathcal{T}-1} a_{\theta(j)}(j)}\right\} .
$$

Portanto, mostramos por indução finita que a Expressão (3.43) é, de fato, verdadeira, o que completa a prova do Teorema 3.4.

Demonstração do Corolário 3.5. A prova deste corolário é trivial. Basta substituir $\theta(\tau)=1, \tau=\mathcal{T}, \ldots, 0$, no Teorema 3.1.

Demonstração do Corolário 3.6. A prova deste corolário é trivial. Basta substituir $\theta(0)=1$ no Teorema 3.2 .

Demonstração do Corolário 3.7. A prova deste corolário é trivial. Basta substituir $\theta(j)=1, j \in\{0, \ldots, \mathcal{T}\}$, no Teorema (3.4).

Demonstração do Corolário 3.8. A prova deste corolário é muito simples. Inicialmente, notemos que:

$$
\Delta X^{*}(\tau+1)=\Delta X(\tau+1)
$$

$$
\begin{aligned}
M^{-1}=\left[E_{\tau}^{P}\left\{k(\tau+1) \Delta X(\tau+1) \Delta X^{*}(\tau+1)\right\}\right]^{-1}= & \frac{1}{E_{\tau}^{P}\left\{k(\tau+1)(\Delta X(\tau+1))^{2}\right\}} .
\end{aligned}
$$

Usando estas relações, a Expressão (3.48) pode ser escrita como:

$$
\begin{aligned}
& H(\tau)=\frac{1}{\frac{k(\tau)}{a(\tau)^{2}}}\left(E_{\tau}^{P}\{(k(\tau+1)-\right. \\
& \left.\left.\left.\frac{E_{\tau}^{P}\{k(\tau+1) \Delta X(\tau+1)\}}{E_{\tau}^{P}\left\{k(\tau+1)(\Delta X(\tau+1))^{2}\right\}} k(\tau+1) \Delta X(\tau+1)\right) \frac{H(\tau+1)}{a(\tau)}\right\}\right) .
\end{aligned}
$$

Como $\frac{k(\tau)}{a(\tau)^{2}}$ é $\mathcal{F}_{\tau^{-}}$-mensurável, a expressão acima pode ser rescrita como:

$H(\tau)=E_{\tau}^{P}\left\{\frac{k(\tau+1)-\frac{E_{\tau}^{P}\{k(\tau+1) \Delta X(\tau+1)\}}{E_{\tau}^{P}\left\{k(\tau+1)(\Delta X(\tau+1))^{2}\right\}} k(\tau+1) \Delta X(\tau+1)}{\frac{k(\tau)}{a(\tau)^{2}}} \frac{H(\tau+1)}{a(\tau)}\right\}$.

Substituindo a Equação (3.58) na expressão acima, obtemos as equações (3.60) e (3.59):

$$
m_{\tau+1, \tau}^{P \rightarrow Q}=\frac{k(\tau+1)-\frac{E_{\tau}^{P}\{k(\tau+1) \Delta X(\tau+1)\}}{E_{\tau}^{P}\left\{k(\tau+1)(\Delta X(\tau+1))^{2}\right\}} k(\tau+1) \Delta X(\tau+1)}{E_{\tau}^{P}\{k(\tau+1)\}-\frac{\left(E_{\tau}^{P}\{k(\tau+1) \Delta X(\tau+1)\}\right)^{2}}{E_{\tau}^{P}\left\{k(\tau+1)(\Delta X(\tau+1))^{2}\right\}}},
$$




$$
H(\tau)=E_{\tau}^{P}\left\{m_{\tau+1, \tau}^{P \rightarrow Q} \frac{H(\tau+1)}{a(\tau)}\right\} .
$$

As outras expressões deste corolário podem ser trivialmente obtidas. Portanto, não há necessidade de desenvolvimentos adicionais.

Demonstração do Corolário 3.9. A prova deste corolário é trivial. Basta substituir $\theta(0)=1$ no Teorema 3.2 .

Demonstração do Corolário 3.10. A prova deste corolário é trivial. Basta substituir $\theta(j)=1, j \in\{0, \ldots, \mathcal{T}\}$, no Teorema 3.4.

Demonstração do Lema 4.1. A Equação (4.30) pode ser rescrita como:

$$
\begin{array}{r}
\Delta X(\tau+1)=\frac{S(\tau)}{S_{0}(\tau)}(e^{(\mu-r) \Delta t_{\tau}} \underbrace{\exp \left\{\sigma \Delta W^{P}(\tau+1)-\frac{1}{2} \sigma^{2} \Delta t_{\tau}\right\}}_{Z_{1}\left(\Delta t_{\tau}\right)} \\
\underbrace{\exp \left\{\left(1-e^{\beta_{Y}+\frac{1}{2} \sigma_{Y}^{2}}\right) \lambda^{P} \Delta t_{\tau}\right\} \prod_{i=1}^{N^{P}\left(\Delta t_{\tau}\right)} e^{Y_{i}^{P}}}_{Z_{2}\left(\Delta t_{\tau}\right)}-1) .
\end{array}
$$

Multiplicando e dividindo a expressão acima por $\left(e^{(\mu-r) \Delta t_{\tau}}-1\right)$, obtemos:

$$
\begin{array}{r}
\Delta X(\tau+1)=\frac{S(\tau)}{S_{0}(\tau)}\left(e^{(\mu-r) \Delta t_{\tau}}-1\right)(\underbrace{\frac{e^{(\mu-r) \Delta t_{\tau}}}{e^{(\mu-r) \Delta t_{\tau}}-1}}_{\alpha_{1}\left(\Delta t_{\tau}\right)} \\
Z_{1}\left(\Delta t_{\tau}\right) Z_{2}\left(\Delta t_{\tau}\right)+\underbrace{\frac{-1}{e^{(\mu-r) \Delta t_{\tau}}-1}}_{\alpha_{0}\left(\Delta t_{\tau}\right)}) .
\end{array}
$$

Definindo:

$$
\begin{aligned}
\alpha_{0}\left(\Delta t_{\tau}\right) & =-\frac{1}{e^{(\mu-r) \Delta t_{\tau}}-1}, \\
\alpha_{1}\left(\Delta t_{\tau}\right) & =\frac{e^{(\mu-r) \Delta t_{\tau}}}{e^{(\mu-r) \Delta t_{\tau}}-1},
\end{aligned}
$$

a Expressão (A.95) pode ser rescrita como:

$$
\Delta X(\tau+1)=\frac{S(\tau)}{S_{0}(\tau)}\left(e^{(\mu-r) \Delta t_{\tau}}-1\right)\left(\alpha_{0}\left(\Delta t_{\tau}\right)+\alpha_{1}\left(\Delta t_{\tau}\right) Z_{1}\left(\Delta t_{\tau}\right) Z_{2}\left(\Delta t_{\tau}\right)\right) .
$$


Para completar a prova, devemos escrever $Z_{2}\left(\Delta t_{\tau}\right)$, destacado na Expressão (A.94), na forma:

$$
Z_{2}\left(\Delta t_{\tau}\right)=e^{\left(\lambda^{P}-\lambda^{Q}\right) \Delta t_{\tau}} \prod_{i=1}^{N^{P}\left(\Delta t_{\tau}\right)} \frac{\lambda^{Q} f^{Q}\left(Y_{i}^{P}\right)}{\lambda^{P} f^{P}\left(Y_{i}^{P}\right)}
$$

onde $f^{P}\left(Y_{i}^{P}\right)$ é a função densidade de probabilidade da variável aleatória $Y_{i}^{P}$ com relação à medida $P$, e $f^{Q}\left(Y_{i}^{P}\right)$ é a função densidade de probabilidade da variável aleatória $Y_{i}^{P}$ com relação a uma outra medida de probabilidade: a medida $Q$.

Sabemos, por hipótese, que $f^{P}\left(Y_{i}^{P}\right)$ corresponde a uma normal com $E_{\tau}^{P}\left\{Y_{i}^{P}\right\}=$ $\beta_{Y}$ e $\sigma_{\tau}^{P}\left\{Y_{i}^{P}\right\}=\sigma_{Y}$ :

$$
f^{P}\left(Y_{i}^{P}\right)=\frac{1}{\sqrt{2 \pi} \sigma_{Y}} \exp \left(-\frac{1}{2}\left(\frac{Y_{i}^{P}-\beta_{Y}}{\sigma_{Y}}\right)^{2}\right) .
$$

Portanto, para que a Igualdade (A.99) seja possível, devemos encontrar $f^{Q}\left(Y_{i}^{P}\right)$ e $\lambda^{Q}$ tais que:

$$
\begin{gathered}
e^{\left(\lambda^{P}-\lambda^{Q}\right) \Delta t_{\tau}} \prod_{i=1}^{N^{P}\left(\Delta t_{\tau}\right)} \frac{\lambda^{Q} f^{Q}\left(Y_{i}^{P}\right)}{\lambda^{P} \underbrace{\frac{1}{\sqrt{2 \pi} \sigma_{Y}} \exp \left(-\frac{1}{2}\left(\frac{Y_{i}^{P}-\beta_{Y}}{\sigma_{Y}}\right)^{2}\right)}_{f^{P}\left(Y_{i}^{P}\right)}}= \\
\underbrace{\exp \left\{\left(1-e^{\beta_{Y}+\frac{1}{2} \sigma_{Y}^{2}}\right) \lambda^{P} \Delta t_{\tau}\right\}^{N^{P}\left(\Delta t_{\tau}\right)} \prod_{i=1}^{Y_{i}^{P}}}_{Z_{2}\left(\Delta t_{\tau}\right)} .
\end{gathered}
$$

Esta equação é satisfeita se:

$$
e^{\left(\lambda^{P}-\lambda^{Q}\right) \Delta t_{\tau}}=\exp \left(\left(1-e^{\beta_{Y}+\frac{1}{2} \sigma_{Y}^{2}}\right) \lambda^{P} \Delta t_{\tau}\right) \Longrightarrow \lambda^{Q}=\lambda^{P} e^{\beta_{Y}+\frac{1}{2} \sigma_{Y}^{2}},
$$

e se:

$\frac{\lambda^{Q} f^{Q}\left(Y_{i}^{P}\right)}{\frac{\lambda^{P}}{\sqrt{2 \pi} \sigma_{Y}} \exp \left(-\frac{1}{2}\left(\frac{Y_{i}^{P}-\beta_{Y}}{\sigma_{Y}}\right)^{2}\right)}=e^{Y_{i}^{P}} \Longrightarrow f^{Q}\left(Y_{i}^{P}\right)=\frac{1}{\sqrt{2 \pi} \sigma_{Y}} e^{-\frac{1}{2}\left(\frac{Y_{i}^{P}-\left(\sigma_{Y}^{2}+\beta_{Y}\right)}{\sigma_{Y}}\right)^{2} .}$

Portanto, $Z_{2}\left(\Delta t_{\tau}\right)$ de fato pode ser escrito na forma da Equação (A.99), com $f^{P}\left(Y_{i}^{P}\right), \lambda^{Q}$ e $f^{Q}\left(Y_{i}^{P}\right)$ dados, respectivamente, por (A.100), (A.102), e (A.103), completando a prova.

Lema A.4. Seja $\Delta X(\tau+1)$ tal como definido no Lema 4.1. Nestas condições, $E_{\tau}^{P}\{\Delta X(\tau+1)\}$ e $E_{\tau}^{P}\left\{(\Delta X(\tau+1))^{2}\right\}$ podem ser expressos como:

$$
E_{\tau}^{P}\{\Delta X(\tau+1)\}=\frac{S(\tau)}{S_{0}(\tau)}\left(e^{(\mu-r) \Delta t_{\tau}}-1\right)
$$




$$
\begin{aligned}
& E_{\tau}^{P}\left\{(\Delta X(\tau+1))^{2}\right\}=\left(\frac{S(\tau)}{S_{0}(\tau)}\right)^{2}\left\{\operatorname { e x p } \left[\left(2 \mu-2 r+\sigma^{2}+\right.\right.\right. \\
& \left.\left.\left.\lambda^{P}\left(1-2 e^{\beta_{Y}+\frac{1}{2} \sigma_{Y}^{2}}+e^{2\left(\sigma_{Y}^{2}+\beta_{Y}\right)}\right)\right) \Delta t_{\tau}\right]-2 \exp \left[(\mu-r) \Delta t_{\tau}\right]+1\right\}
\end{aligned}
$$

Demonstraçãao. Calculemos inicialmente $E_{\tau}^{P}\{\Delta X(\tau+1)\}$. Como os processos $Z_{1}\left(\Delta t_{\tau}\right)$ e $Z_{2}\left(\Delta t_{\tau}\right)$ dados, respectivamente, pelas Equações (4.34) e (4.35) são independentes, temos:

$$
\begin{aligned}
& E_{\tau}^{P}\{\Delta X(\tau+1)\}= \\
& \frac{S(\tau)}{S_{0}(\tau)}\left(e^{(\mu-r) \Delta t_{\tau}}-1\right)\left(\alpha_{0}\left(\Delta t_{\tau}\right)+\alpha_{1}\left(\Delta t_{\tau}\right) E_{\tau}^{P}\left\{Z_{1}\left(\Delta t_{\tau}\right)\right\} E_{\tau}^{P}\left\{Z_{2}\left(\Delta t_{\tau}\right)\right\}\right)
\end{aligned}
$$

Sendo $\Theta$ uma variável aleatória com distribuição normal de média $\mu$ e variância $\sigma^{2}$, i.e., $\Theta N\left(\mu_{\Theta}, \sigma_{\Theta}^{2}\right)$, temos:

$$
E\left\{e^{u \Theta}\right\}=\exp \left(u \mu_{\Theta}+\frac{1}{2} u^{2} \sigma_{\Theta}^{2}\right)
$$

Aplicando esta expressão ao cálculo de $E_{\tau}^{P}\left\{Z_{1}\left(\Delta t_{\tau}\right)\right\}$, obtemos:

$$
\begin{gathered}
E_{\tau}^{P}\left\{Z_{1}\left(\Delta t_{\tau}\right)\right\}=E_{\tau}^{P}\{\exp \underbrace{\left(\sigma \Delta W^{P}(\tau+1)-\frac{1}{2} \sigma^{2} \Delta t_{\tau}\right)}_{1 \Theta}\}= \\
\exp (\underbrace{-\frac{1}{2} \sigma^{2} \Delta t_{\tau}}_{1 \mu_{\Theta}}+\frac{1}{2} \underbrace{\sigma^{2} \Delta t_{\tau}}_{1^{2} \sigma_{\Theta}^{2}})=1 .
\end{gathered}
$$

Antes de efetuarmos o cálculo de $E_{\tau}^{P}\left\{Z_{2}\left(\Delta t_{\tau}\right)\right\}$, consideremos o seguinte processo:

$$
\left(J\left(\Delta t_{\tau}\right)\right)^{m}=\left(\prod_{i=1}^{N^{P}\left(\Delta t_{\tau}\right)} e^{Y_{i}^{P}}\right)^{m} .
$$


O valor esperado de $\left(J\left(\Delta t_{\tau}\right)\right)^{m}$ é dado por:

$$
\begin{aligned}
& E_{\tau}^{P}\left\{\left(J\left(\Delta t_{\tau}\right)\right)^{m}\right\}=E_{\tau}^{P}\left\{\left(\prod_{i=1}^{N^{P}\left(\Delta t_{\tau}\right)} e^{Y_{i}^{P}}\right)^{m}\right\}= \\
& E_{\tau}^{P}\left\{E_{\tau}^{P}\left\{\prod_{i=1}^{N^{P}\left(\Delta t_{\tau}\right)} e^{m Y_{i}^{P}} \mid N\left[\Delta t_{\tau}\right]=k\right\}\right\}=E_{\tau}^{P}\left\{\prod_{i=1}^{k}\left(E_{\tau}^{P}\left\{e^{m Y_{i}^{P}}\right\}\right)\right\}= \\
& E_{\tau}^{P}\left\{\prod_{i=1}^{k} e^{m \beta_{Y}+\frac{1}{2} m^{2} \sigma_{Y}^{2}}\right\}=E_{\tau}^{P}\left\{\left(e^{m \beta_{Y}+\frac{1}{2} m^{2} \sigma_{Y}^{2}}\right)^{k}\right\}= \\
& \sum_{k=0}^{\infty} \frac{\left(e^{m \beta_{Y}+\frac{1}{2} m^{2} \sigma_{Y}^{2}}\right)^{k}\left(e^{-\lambda^{P} \Delta t_{\tau}}\left(\lambda^{P} \Delta t_{\tau}\right)^{k}\right)}{k !}= \\
& e^{-\lambda^{P} \Delta t} \sum_{k=0}^{\infty} \frac{\left(\lambda^{P} \Delta t_{\tau} e^{m \beta_{Y}+\frac{1}{2} m^{2} \sigma_{Y}^{2}}\right)^{k}}{k !}= \\
& e^{-\lambda^{P} \Delta t_{\tau}} \exp \left(\lambda^{P} \Delta t_{\tau} e^{m \beta_{Y}+\frac{1}{2} m^{2} \sigma_{Y}^{2}}\right)=\exp \left[\lambda^{P} \Delta t_{\tau}\left(e^{m \beta_{Y}+\frac{1}{2} m^{2} \sigma_{Y}^{2}}-1\right)\right] \text {. }
\end{aligned}
$$

Portanto, fazendo $m=1$ na expressão acima, obtemos:

$$
E^{P}\left\{J\left(\Delta t_{\tau}\right)\right\}=E^{P}\left\{\prod_{i=1}^{N^{P}\left(\Delta t_{\tau}\right)} e^{Y_{i}^{P}}\right\}=\exp \left[\lambda^{P} \Delta t_{\tau}\left(e^{\beta_{Y}+\frac{1}{2} \sigma_{Y}^{2}}-1\right)\right]
$$

Da demonstração do Lema 4.1 (Equação (A.94)), sabemos que $Z_{2}\left(\Delta t_{\tau}\right)$, dado pela Equação (4.35), também pode ser escrito como:

$$
Z_{2}\left(\Delta t_{\tau}\right)=\exp \left[\lambda^{P} \Delta t_{\tau}\left(1-e^{\beta_{Y}+\frac{1}{2} \sigma_{Y}^{2}}\right)\right] \prod_{i=1}^{N^{P}\left(\Delta t_{\tau}\right)} e^{Y_{i}^{P}}
$$

Usando então o resultado da Equação (A.111), podemos finalmente calcular $E_{\tau}^{P}\left\{Z_{2}\left(\Delta t_{\tau}\right)\right\}$ :

$$
E_{\tau}^{P}\left\{Z_{2}\left(\Delta t_{\tau}\right)\right\}=\exp \left[\lambda^{P} \Delta t_{\tau}\left(1-e^{\beta_{Y}+\frac{1}{2} \sigma_{Y}^{2}}\right)\right] E_{\tau}^{P}\left\{\prod_{i=1}^{N^{P}\left(\Delta t_{\tau}\right)} e^{Y_{i}^{P}}\right\}=1 .
$$

Portanto, substituindo na Expressão (A.106) os resultados obtidos em (A.108) e (A.113), e usando as equações (4.32) e (4.33), obtemos:

$$
\begin{array}{r}
E_{\tau}^{P}\{\Delta X(\tau+1)\}=\frac{S(\tau)}{S_{0}(\tau)}\left(e^{(\mu-r) \Delta t_{\tau}}-1\right)\left(\alpha_{0}\left(\Delta t_{\tau}\right)+\alpha_{1}\left(\Delta t_{\tau}\right)\right)= \\
\frac{S(\tau)}{S_{0}(\tau)}\left(e^{(\mu-r) \Delta t_{\tau}}-1\right) .
\end{array}
$$


Calculemos agora $E_{\tau}^{P}\left\{(\Delta X(\tau+1))^{2}\right\}$. Usando a Equação (4.31), obtemos para $(\Delta X(\tau+1))^{2}$ :

$$
\begin{gathered}
(\Delta X(\tau+1))^{2}=\left(\frac{S(\tau)}{S_{0}(\tau)}\right)^{2}\left(e^{(\mu-r) \Delta t_{\tau}}-1\right)^{2} \\
\left(\alpha_{0}\left(\Delta t_{\tau}\right)^{2}+2 \alpha_{0}\left(\Delta t_{\tau}\right) \alpha_{1}\left(\Delta t_{\tau}\right) Z_{1}\left(\Delta t_{\tau}\right) Z_{2}\left(\Delta t_{\tau}\right)+\alpha_{1}\left(\Delta t_{\tau}\right)^{2} Z_{1}\left(\Delta t_{\tau}\right)^{2} Z_{2}\left(\Delta t_{\tau}\right)^{2}\right) .
\end{gathered}
$$

Calculando o valor esperado desta expressão, obtemos:

$$
\begin{aligned}
& E_{\tau}^{P}\left\{(\Delta X(\tau+1))^{2}\right\}=\left(\frac{S(\tau)}{S_{0}(\tau)}\right)^{2}\left(e^{(\mu-r) \Delta t_{\tau}}-1\right)^{2} \\
&\left(\alpha_{0}\left(\Delta t_{\tau}\right)^{2}+2 \alpha_{0}\left(\Delta t_{\tau}\right) \alpha_{1}\left(\Delta t_{\tau}\right) E_{\tau}^{P}\left\{Z_{1}\left(\Delta t_{\tau}\right)\right\} E_{\tau}^{P}\left\{Z_{2}\left(\Delta t_{\tau}\right)\right\}+\right. \\
&\left.\alpha_{1}\left(\Delta t_{\tau}\right)^{2} E_{\tau}^{P}\left\{Z_{1}\left(\Delta t_{\tau}\right)^{2}\right\} E_{\tau}^{P}\left\{Z_{2}\left(\Delta t_{\tau}\right)^{2}\right\}\right)
\end{aligned}
$$

Usando a Expressão (A.107) com $u=2$ obtemos $E_{\tau}^{P}\left\{Z_{1}\left(\Delta t_{\tau}\right)^{2}\right\}$ :

$$
\begin{gathered}
E_{\tau}^{P}\left\{Z_{1}\left(\Delta t_{\tau}\right)^{2}\right\}=E_{\tau}^{P}\{\exp \underbrace{\left(2 \sigma \Delta \mathrm{W}^{\mathrm{P}}(\tau+1)-\sigma^{2} \Delta \mathrm{t}_{\tau}\right)}_{2 \Theta}\}= \\
\exp (\underbrace{-\sigma^{2} \Delta t_{\tau}}_{2 \mu_{\Theta}}+\frac{1}{2} \underbrace{4 \sigma^{2} \Delta t_{\tau}}_{2^{2} \sigma_{\Theta}^{2}})=e^{\sigma^{2} \Delta t_{\tau}} .
\end{gathered}
$$

Usando a Expressão (A.110) com $u=2$ obtemos $E_{\tau}^{P}\left\{Z_{2}\left(\Delta t_{\tau}\right)^{2}\right\}$ :

$$
\begin{gathered}
E_{\tau}^{P}\left\{Z_{2}\left(\Delta t_{\tau}\right)^{2}\right\}=E_{\tau}^{P}\left\{\exp \left[2 \lambda^{P} \Delta t_{\tau}\left(1-e^{\beta_{Y}+\frac{1}{2} \sigma_{Y}^{2}}\right)\right] \prod_{i=1}^{N^{P}\left(\Delta t_{\tau}\right)} e^{2 Y_{i}^{P}}\right\}= \\
\exp \left[2 \lambda^{P} \Delta t_{\tau}\left(1-e^{\beta_{Y}+\frac{1}{2} \sigma_{Y}^{2}}\right)\right] E_{\tau}^{P}\left\{\prod_{i=1}^{N^{P}\left(\Delta t_{\tau}\right)} e^{2 Y_{i}^{P}}\right\}= \\
\exp \left[2 \lambda^{P} \Delta t_{\tau}\left(1-e^{\beta_{Y}+\frac{1}{2} \sigma_{Y}^{2}}\right)\right] \exp \left[\lambda^{P} \Delta t_{\tau}\left(e^{2 \beta_{Y}+\frac{1}{2} 2^{2} \sigma_{Y}^{2}}-1\right)\right]= \\
\exp \left[\lambda^{P}\left(1-2 e^{\frac{1}{2} \sigma_{Y}^{2}+\beta_{Y}}+e^{2\left(\sigma_{Y}^{2}+\beta_{Y}\right)}\right) \Delta t_{\tau}\right] .
\end{gathered}
$$

Portanto, substituindo os resultados encontrados na Equação (A.116), e usando novamente as equações (4.32) e (4.33), obtemos:

$$
\begin{aligned}
& E_{\tau}^{P}\left\{(\Delta X(\tau+1))^{2}\right\}= \\
& \left(\frac{S(\tau)}{S_{0}(\tau)}\right)^{2}\left\{\exp \left[\left(2 \mu-2 r+\sigma^{2}+\lambda^{P}\left(1-2 e^{\beta_{Y}+\frac{1}{2} \sigma_{Y}^{2}}+e^{2\left(\sigma_{Y}^{2}+\beta_{Y}\right)}\right)\right) \Delta t_{\tau}\right]-\right. \\
& \left.2 \exp \left[(\mu-r) \Delta t_{\tau}\right]+1\right\}, \quad \text { (A.119) }
\end{aligned}
$$

o que completa a prova. 
Lema A.5. Seja $\Delta X(\tau+1)$ um processo estocástico tal como definido no Lema 4.1. O processo recursivo $k(\tau)$ dado por:

$$
k(\tau)=E_{\tau}^{P}\{k(\tau+1)\}-\frac{\left(E_{\tau}^{P}\{k(\tau+1) \Delta X(\tau+1)\}\right)^{2}}{E_{\tau}^{P}\left\{k(\tau+1)(\Delta X(\tau+1))^{2}\right\}} ; k(\mathcal{T})=1
$$

pode ser expresso como:

$$
k(\tau)=\prod_{i=\tau}^{(\mathcal{T}-1)} \theta(i),
$$

onde:

$$
\begin{gathered}
\theta(i)=1-\frac{\left(e^{(\mu-r) \Delta t_{i}}-1\right)^{2}}{A} \\
A=\exp \left[\left(2 \mu-2 r+\sigma^{2}+\lambda^{P}\left(1-2 e^{\beta_{Y}+\frac{1}{2} \sigma_{Y}^{2}}+e^{2\left(\sigma_{Y}^{2}+\beta_{Y}\right)}\right)\right) \Delta t_{i}\right]- \\
2 \exp \left[(\mu-r) \Delta t_{i}\right]+1 .
\end{gathered}
$$

Demonstração. A prova deste lema é feita por indução finita. Suponhamos:

$$
k(\tau+1)=\prod_{i=\tau+1}^{(\mathcal{T}-1)} \theta(i)
$$

No instante $\mathcal{T}$ (i.e., $\tau=\mathcal{T}-1$ ):

$$
k(\mathcal{T})=\prod_{i=\mathcal{T}}^{\mathcal{T}-1} \theta(i)=1 .
$$

No instante $\tau$ :

$$
\begin{aligned}
k(\tau)=E_{\tau}^{P}\{k(\tau+1)\}-\frac{\left(E_{\tau}^{P}\{k(\tau+1) \Delta X(\tau+1)\}\right)^{2}}{E_{\tau}^{P}\left\{k(\tau+1)(\Delta X(\tau+1))^{2}\right\}}= \\
E_{\tau}^{P}\left\{\prod_{i=\tau+1}^{(\mathcal{T}-1)} \theta(i)\right\}-\frac{\left(E_{\tau}^{P}\left\{\prod_{i=\tau+1}^{(\mathcal{T}-1)} \theta(i) \Delta X(\tau+1)\right\}\right)^{2}}{E_{\tau}^{P}\left\{\prod_{i=\tau+1}^{(\mathcal{T}-1)} \theta(i)(\Delta X(\tau+1))^{2}\right\}} .
\end{aligned}
$$

O processo $\prod_{i=\tau+1}^{(\mathcal{T}-1)} \theta(i)$ é $\mathcal{F}_{\tau}$-mensurável. Portanto:

$$
k(\tau)=\left(\prod_{i=\tau+1}^{(\mathcal{T}-1)} \theta(i)\right)\left(1-\frac{\left(E_{\tau}^{P}\{\Delta X(\tau+1)\}\right)^{2}}{E_{\tau}^{P}\left\{(\Delta X(\tau+1))^{2}\right\}}\right) .
$$

Usando os resultados do Lema A.4, podemos escrever:

$$
\frac{\left(E_{\tau}^{P}\{\Delta X(\tau+1)\}\right)^{2}}{E_{\tau}^{P}\left\{(\Delta X(\tau+1))^{2}\right\}}=\frac{\left(e^{(\mu-r) \Delta t_{\tau}}-1\right)^{2}}{A},
$$




$$
\begin{array}{r}
A=\exp \left(2 \mu-2 r+\sigma^{2}+\lambda^{P}\left(1-2 e^{\beta_{Y}+\frac{1}{2} \sigma_{Y}^{2}}+e^{2\left(\sigma_{Y}^{2}+\beta_{Y}\right)}\right)\right) \Delta t_{\tau}- \\
2 \exp \left[(\mu-r) \Delta t_{\tau}\right]+1 .
\end{array}
$$

Portanto:

$$
k(\tau)=(\prod_{i=\tau+1}^{(\mathcal{T}-1)} \theta(i) \underbrace{\left(1-\frac{\left(e^{(\mu-r) \Delta t_{\tau}}-1\right)^{2}}{A}\right)}_{\theta(\tau)}=\prod_{i=\tau}^{(\mathcal{T}-1)} \theta(i) .
$$

Isso completa a prova por indução finita.

Lema A.6. O produtório $\prod_{j=1}^{n}\left(a_{j}+b_{j}\right)$ pode ser escrito como:

$$
\begin{aligned}
\prod_{j=1}^{n}\left(a_{j}+b_{j}\right)= & \sum_{p=0}^{n} \sum_{l=1}^{\left(\begin{array}{c}
n \\
p
\end{array}\right)}\left[\left(\prod_{j \in C_{p, l}^{n}} a_{j}\right)\left(\prod_{j \in C_{n-p, s}^{n}} b_{j}\right)\right]= \\
& \sum_{p=0}^{n} \sum_{l=1}^{\left(\begin{array}{l}
n \\
p
\end{array}\right)}\left[\left(\prod_{j=1}^{n}\left(a_{j}\right)^{\mathbf{1}_{p, l}^{n}(j)}\right)\left(\prod_{j=1}^{n}\left(b_{j}\right)^{\mathbf{1}_{C_{n-p, s}^{n}}(j)}\right)\right]
\end{aligned}
$$

Onde:

- $s=\left[\left(\begin{array}{l}n \\ p\end{array}\right)-l+1\right]$;

- $C_{p}^{n}$ é o conjunto cujos elementos $C_{p, l}^{n}\left(1 \leq l \leq\left(\begin{array}{l}n \\ p\end{array}\right)\right)$ são subconjuntos, dispostos em ordem lexicográfica, formados por $p(0 \leq p \leq n)$ elementos extraídos do conjunto $\{1, \cdots, n\}$;

- $C_{n-p}^{n}$ é o conjunto cujos elementos $C_{n-p, s}^{n}\left(1 \leq s \leq\left(\begin{array}{c}n \\ n-p\end{array}\right)=\left(\begin{array}{l}n \\ p\end{array}\right)\right)$ são subconjuntos, dispostos em ordem lexicográfica, formados por $n-p(0 \leq$ $p \leq n)$ elementos extraídos do conjunto $\{1, \cdots, n\}$

$O$ conjunto $C_{p}^{n}$ possui $\left(\begin{array}{l}n \\ p\end{array}\right)=\frac{n !}{p !(n-p) !}$ elementos, e cada um desses elementos é um conjunto contendo $p$ elementos extraídos do conjunto $\{1, \cdots, n\}$. $O$ conjunto $C_{n-p}^{n}$ também possui $\left(\begin{array}{c}n \\ n-p\end{array}\right)=\frac{n !}{p !(n-p) !}$ elementos, e cada um desses elementos é um conjunto contendo $n-p$ elementos extraídos do conjunto $\{1, \cdots, n\}$.

Demonstração. Seja a prova por indução finita em $n$. 
Para $n=1$, temos:

$$
\begin{aligned}
& \sum_{p=0}^{n} \sum_{l=1}^{\left(\begin{array}{l}
n \\
p
\end{array}\right)}\left[\left(\prod_{j \in C_{p, l}^{n}} a_{j}\right)\left(\prod_{j \in C_{n-p, s}^{n}} b_{j}\right)\right]= \\
& \sum_{p=0}^{1} \sum_{l=1}^{\left(\begin{array}{l}
1 \\
p
\end{array}\right)}\left[\left(\prod_{j \in C_{p, l}^{1}} a_{j}\right)\left(\prod_{j \in C_{1-p, s}^{1}} b_{j}\right)\right]= \\
& \underbrace{\sum_{l=1}^{\left(\begin{array}{l}
1 \\
0
\end{array}\right)}\left[\left(\prod_{j \in C_{0, l}^{1}} a_{j}\right)\left(\prod_{j \in C_{1-0, s}^{1}} b_{j}\right)\right]}_{p=0}+\underbrace{\sum_{l=1}^{\sum_{1}^{1}}\left[\left(\prod_{j \in C_{1, l}^{1}} a_{j}\right)\left(\prod_{j \in C_{1-1, s}^{1}} b_{j}\right]\right.}_{p=1}=
\end{aligned}
$$

Assumindo que a Expressão (A.131) seja válida para $n$, devemos provar que ela também é válida para $n+1$, i.e.:

$$
\prod_{j=1}^{n+1}\left(a_{j}+b_{j}\right)=\sum_{p=0}^{n+1} \sum_{l=1}^{\left(\begin{array}{c}
n+1 \\
p
\end{array}\right)}\left[\left(\prod_{j \in C_{p, l}^{n+1}} a_{j}\right)\left(\prod_{j \in C_{n+1}^{n+1}-s, s} b_{j}\right)\right] .
$$

Inicialmente, notemos que os elementos dos conjuntos $C_{p}^{n}$ e $C_{n-p}^{n}$ são dispostos em ordem lexicográfica. Assim, por construção, para qualquer $l, 1 \leq l \leq\left(\begin{array}{l}n \\ p\end{array}\right)$ e $s=\left[\left(\begin{array}{l}n \\ p\end{array}\right)-l-1\right]$, os conjuntos $C_{p, l}^{n}$ e $C_{n-p, s}^{n}$ formam uma partição do conjunto $\{1,2, \ldots n\}$, ou seja, $C_{p, l}^{n} \cup C_{n-p, s}^{n}=\{1,2, \ldots, n\}$ e $C_{p, l}^{n} \cap C_{n-p, s}^{n}=\emptyset$. Consideremos a soma:

$$
S=\sum_{l=1}^{\left(\begin{array}{c}
n+1 \\
p
\end{array}\right)}\left[\left(\prod_{j \in C_{p, l}^{n+1}} a_{j}\right)\left(\prod_{j \in C_{n+1-p, s}^{n+1}} b_{j}\right)\right]
$$

A soma $S$ é composta por $\left(\begin{array}{c}n+1 \\ p\end{array}\right)$ parcelas. Cada parcela da soma $S$ é um produto de $n+1$ fatores. Como os conjuntos $C_{p, l}^{n+1}$ e $C_{n+1-p, s}^{n+1}$ formam uma partição do conjunto $\{1,2, \ldots, n, n+1\}$, então cada parcela da soma $S$ ou possui o fator $a_{n+1}$ ou possui o fator $b_{n+1}$. Dessa forma, é possível rescrever a soma $S$ evidenciando os termos $a_{n+1}$ e $b_{n+1}$ :

$$
\begin{aligned}
& S=\sum_{l=1}^{\left(\begin{array}{c}
n+1 \\
p
\end{array}\right)}\left[\left(\prod_{j \in C_{p, l}^{n+1}} a_{j}\right)\right.\left.\left(\prod_{j \in C_{n+1-p, s}^{n+1}} b_{j}\right)\right]= \\
& b_{n+1} \sum_{l=1}^{\left(\begin{array}{c}
n \\
p
\end{array}\right)}\left[\left(\prod_{j \in C_{p, l}^{n}} a_{j}\right)\left(\prod_{j \in C_{n-p, s}^{n}} b_{j}\right)\right]+ \\
& a_{n+1} \sum_{l=1}^{\left(\begin{array}{c}
n \\
p-1
\end{array}\right)}\left[\left(\prod_{j \in C_{p-1, l}^{n}} a_{j}\right)\left(\prod_{j \in C_{n-(p-1), s}^{n}} b_{j}\right)\right] .
\end{aligned}
$$


Notemos que a soma $S$, que continha $\left(\begin{array}{c}n+1 \\ p\end{array}\right)$ parcelas, foi dividida em duas somas com $\left(\begin{array}{c}n \\ p\end{array}\right)$ e $\left(\begin{array}{c}n \\ p-1\end{array}\right)$ parcelas. Com efeito, $\left(\begin{array}{c}n+1 \\ p\end{array}\right)=\left(\begin{array}{c}n \\ p\end{array}\right)+\left(\begin{array}{c}n \\ p-1\end{array}\right)$.

Considerando esse resultado parcial, rescrevemos a expressão abaixo, separando as parcelas $p=0$ e $p=n+1$ :

$$
\begin{aligned}
& \sum_{p=0}^{n+1} \sum_{l=1}^{\substack{n+1 \\
p}}\left[\left(\prod_{j \in C_{p, l}^{n+1}} a_{j}\right)\left(\prod_{j \in C_{n+1-p, s}^{n+1}} b_{j}\right)\right]= \\
& \underbrace{\sum_{l=1}^{\left(\begin{array}{c}
n+1 \\
0
\end{array}\right)}\left[\left(\prod_{j \in C_{0, l}^{n+1}} a_{j}\right)\left(\prod_{j \in C_{n+1-0, s}^{n+1}} b_{j}\right)\right]}_{T_{1}}+ \\
& \underbrace{\sum_{p=1}^{n} \sum_{l=1}^{\left(\begin{array}{c}
n+1 \\
p
\end{array}\right)}\left[\left(\prod_{j \in C_{p, l}^{n+1}} a_{j}\right)\left(\prod_{j \in C_{n+1-p, s}^{n+1}} b_{j}\right)\right]}_{T_{2}} \\
& +\underbrace{\sum_{l=1}^{\left(\begin{array}{c}
n+1 \\
n+1
\end{array}\right)}\left[\left(\prod_{j \in C_{n+1, l}^{n+1}} a_{j}\right)\left(\prod_{j \in C_{n+1-(n+1), s}^{n+1}} b_{j}\right)\right]}_{T_{3}} .
\end{aligned}
$$

Temos que:

$$
\begin{gathered}
T_{1}=\sum_{l=1}^{\left(\begin{array}{c}
n+1 \\
0
\end{array}\right)}\left[\left(\prod_{j \in C_{0, l}^{n+1}} a_{j}\right)\left(\prod_{j \in C_{n+1-0, s}^{n+1}} b_{j}\right)\right]= \\
b_{n+1} \sum_{l=1}^{\left(\begin{array}{c}
n \\
0
\end{array}\right)}\left[\left(\prod_{j \in C_{0, l}^{n}} a_{j}\right)\left(\prod_{j \in C_{n-0, s}^{n}} b_{j}\right)\right], \\
T_{3}=a_{n+1} \sum_{l=1}^{\left(\begin{array}{c}
n \\
n
\end{array}\right)}\left[\left(\prod_{j \in C_{n, l}^{n}} a_{j}\right)\left(\prod_{j \in C_{n-n, s}^{n}} b_{j}\right)\right] .
\end{gathered}
$$


Considerando o resultado da Equação (A.135):

$$
\begin{gathered}
\sum_{p=1}^{n} \sum_{l=1}^{\left(\begin{array}{c}
n+1 \\
p
\end{array}\right)}\left[\left(\prod_{j \in C_{p, l}^{n+1}} a_{j}\right)\left(\prod_{j \in C_{n+1-p, s}^{n+1}} b_{j}\right)\right]= \\
\sum_{p=1}^{n} b_{n+1} \sum_{l=1}^{\left(\begin{array}{c}
n \\
p
\end{array}\right)}\left[\left(\prod_{j \in C_{p, l}^{n}} a_{j}\right)\left(\prod_{j \in C_{n-p, s}^{n}} b_{j}\right)\right]+ \\
\sum_{p=1}^{n} a_{n+1} \sum_{l=1}^{\left(\begin{array}{c}
n \\
p-1
\end{array}\right)}\left[\left(\prod_{j \in C_{p-1, l}^{n}} a_{j}\right)\left(\prod_{j \in C_{n-(p-1), s}^{n}} b_{j}\right)\right]= \\
b_{n+1} \sum_{p=1}^{n} \sum_{l=1}^{\left(\begin{array}{c}
n \\
p
\end{array}\right)}\left[\left(\prod_{j \in C_{p, l}^{n}} a_{j}\right)\left(\prod_{j \in C_{n-p, s}^{n}} b_{j}\right)\right]+ \\
a_{n+1} \sum_{p=0}^{n-1} \sum_{l=1}^{\left(\begin{array}{l}
n \\
p
\end{array}\right)}\left[\left(\prod_{j \in C_{p, l}^{n}} a_{j}\right)\left(\prod_{j \in C_{n-p, s}^{n}} b_{j}\right)\right] .
\end{gathered}
$$

Utilizando (A.137), (A.138) e (A.139), rescrevemos a Equação (A.136):

$$
\begin{aligned}
\sum_{p=0}^{n+1} \sum_{l=1}^{\left(\begin{array}{c}
n+1 \\
p
\end{array}\right)}\left[\left(\prod_{j \in C_{p, l}^{n+1}} a_{j}\right)\left(\prod_{j \in C_{n+1-p, s}^{n+1}} b_{j}\right)\right]= \\
b_{n+1} \sum_{l=1}^{\left(\begin{array}{l}
n \\
0
\end{array}\right)}\left[\left(\prod_{j \in C_{0, l}^{n}} a_{j}\right)\left(\prod_{j \in C_{n-0, s}^{n}} b_{j}\right)\right]+ \\
b_{n+1} \sum_{p=1}^{n} \sum_{l=1}^{\left(\begin{array}{c}
n \\
p
\end{array}\right)}\left[\left(\prod_{j \in C_{p, l}^{n}} a_{j}\right)\left(\prod_{j \in C_{n-p, s}^{n}} b_{j}\right)+\right. \\
a_{n+1} \sum_{p=0}^{n-1} \sum_{l=1}^{\left(\begin{array}{c}
n \\
p
\end{array}\right)}\left[\left(\prod_{j \in C_{p, l}^{n}} a_{j}\right)\left(\prod_{j \in C_{n-p, s}^{n}} b_{j}\right)\right]+ \\
a_{n+1} \sum_{l=1}^{\left(\begin{array}{c}
n \\
n
\end{array}\right)}\left[\left(\prod_{j \in C_{n, l}^{n}} a_{j}\right)\left(\prod_{j \in C_{n-n, s}^{n}} b_{j}\right)\right] .
\end{aligned}
$$


Reagrupando termos, esta expressão se torna:

$$
\begin{aligned}
& \sum_{p=0}^{n+1} \sum_{l=1}^{\left(\begin{array}{c}
n+1 \\
p
\end{array}\right)}\left[\left(\prod_{j \in C_{p, l}^{n+1}} a_{j}\right)\right.\left.\left(\prod_{j \in C_{n+1-p, s}^{n+1}} b_{j}\right)\right]= \\
& b_{n+1} \sum_{p=0}^{n} \sum_{l=1}^{\left(\begin{array}{c}
n \\
p
\end{array}\right)}\left[\left(\prod_{j \in C_{p, l}^{n}} a_{j}\right)\left(\prod_{j \in C_{n-p, s}^{n}} b_{j}\right)+\right. \\
& a_{n+1} \sum_{p=0}^{n} \sum_{l=1}^{\left(\begin{array}{c}
n \\
p
\end{array}\right)}\left[\left(\prod_{j \in C_{p, l}^{n}} a_{j}\right)\left(\prod_{j \in C_{n-p, s}^{n}} b_{j}\right)\right],
\end{aligned}
$$

que pode ser finalmente simplificada para:

$$
\begin{aligned}
\sum_{p=0}^{n+1} \sum_{l=1}^{\left(\begin{array}{c}
n+1 \\
p
\end{array}\right)}\left[\left(\prod_{j \in C_{p, l}^{n+1}} a_{j}\right)\left(\prod_{j \in C_{n+1-p, s}^{n+1}} b_{j}\right)\right]= \\
\left(a_{n+1}+b_{n+1}\right) \underbrace{\sum_{p=0}^{n} \sum_{l=1}^{\left(\begin{array}{c}
n \\
p
\end{array}\right)}\left[\left(\prod_{j \in C_{p, l}^{n}} a_{j}\right)\left(\prod_{j \in C_{n-p, s}^{n}} b_{j}\right)\right.}_{\prod_{j=1}^{n}\left(a_{j}+b_{j}\right)} .
\end{aligned}
$$

Pela hipótese da indução (Equação (A.131)):

$$
\prod_{j=1}^{n}\left(a_{j}+b_{j}\right)=\sum_{p=0}^{n} \sum_{l=1}^{\left(\begin{array}{l}
n \\
p
\end{array}\right)}\left[\left(\prod_{j \in C_{p, l}^{n}} a_{j}\right)\left(\prod_{j \in C_{n-p, s}^{n}} b_{j}\right)\right] .
$$

Portanto:

$$
\begin{aligned}
\sum_{p=0}^{n+1} \sum_{l=1}^{\left(\begin{array}{c}
n+1 \\
p
\end{array}\right)}\left[\left(\prod_{j \in C_{p, l}^{n+1}} a_{j}\right)\right. & \left.\left(\prod_{j \in C_{n+1}^{n+1}-p, s} b_{j}\right)\right]= \\
\left(a_{n+1}+b_{n+1}\right) \prod_{j=1}^{n}\left(a_{j}+b_{j}\right) & =\prod_{j=1}^{n+1}\left(a_{j}+b_{j}\right),
\end{aligned}
$$

o que completa a prova.

\section{Exemplo de Aplicação}

Representemos $C_{p}^{n}$ com a seguinte notação: $C_{p}^{n}=\operatorname{PSubsets}[\{1, \cdots, n\}, p]$. Exemplos de PSubsets para o conjunto $\{1,2,3,4\}$ :

$$
\begin{gathered}
C_{0}=\text { PSubsets }[\{1,2,3,4\}, 0]=\{\{\}\} \\
C_{1}=\text { PSubsets }[\{1,2,3,4\}, 1]=\{\{1\},\{2\},\{3\},\{4\}\}
\end{gathered}
$$




$$
\begin{gathered}
C_{2}=\text { PSubsets }[\{1,2,3,4\}, 2]=\{\{1,2\},\{1,3\},\{1,4\},\{2,3\},\{2,4\},\{3,4\}\} \\
C_{3}=\text { PSubsets }[\{1,2,3,4\}, 3]=\{\{1,2,3\},\{1,2,4\},\{1,3,4\},\{2,3,4\}\} \\
\qquad C_{4}=\operatorname{PSubsets}[\{1,2,3,4\}, 4]=\{\{1,2,3,4\}\} \\
\text { Exemplo de } \prod_{j=1}^{n}\left(a_{j}+b_{j}\right): \\
\prod_{j=1}^{3}\left(a_{j}+b_{j}\right)=a_{1} a_{2} a_{3}+a_{1} a_{2} b_{3}+a_{1} a_{3} b_{2}+a_{2} a_{3} b_{1}+a_{1} b_{2} b_{3}+a_{2} b_{1} b_{3}+a_{3} b_{1} b_{2}+b_{1} b_{2} b_{3} .
\end{gathered}
$$

Vejamos como fica a fórmula:

$$
\sum_{p=0}^{3} \sum_{l=1}^{\left(\begin{array}{l}
3 \\
p
\end{array}\right)}\left[\left(\prod_{j \in C_{p, l}^{3}} a_{j}\right)\left(\prod_{j \in C_{3-p, s}^{3}} b_{j}\right)\right], s=\left[\left(\begin{array}{l}
3 \\
p
\end{array}\right)-l+1\right] .
$$

Expandindo esta fórmula, obtemos:

$$
\begin{gathered}
\sum_{p=0}^{3} \sum_{l=1}^{\left(\begin{array}{l}
3 \\
p
\end{array}\right)}\left[\left(\prod_{j \in C_{p, l}^{3}} a_{j}\right)\left(\prod_{j \in C_{3-p, s}^{3}} b_{j}\right)\right]= \\
\sum_{l=1}^{\left(\begin{array}{l}
3 \\
0
\end{array}\right)}\left[\left(\prod_{j \in C_{0, l}^{3}} a_{j}\right)\left(\prod_{j \in C_{3,2-l}^{3}} b_{j}\right)\right]+ \\
\sum_{l=1}^{\left(\begin{array}{l}
3 \\
1
\end{array}\right)}\left[\left(\prod_{j \in C_{1, l}^{3}} a_{j}\right)\left(\prod_{j \in C_{2,4-l}^{3}} b_{j}\right)\right]+\sum_{l=1}^{\left(\begin{array}{l}
3 \\
2
\end{array}\right)}\left[\left(\prod_{j \in C_{2, l}^{3}} a_{j}\right)\left(\prod_{j \in C_{1,4-l}^{3}} b_{j}\right)\right]+ \\
\sum_{l=1}^{\left(\begin{array}{l}
3 \\
3
\end{array}\right)}\left[\left(\prod_{j \in C_{3, l}^{3}} a_{j}\right)\left(\prod_{j \in C_{0,2-l}^{3}} b_{j}\right)\right] .
\end{gathered}
$$

Calculemos os $C_{p}^{n}, 0 \leq p \leq 3$ e os $C_{p, j}, 0 \leq p \leq 3,1 \leq l \leq 3$ para o conjunto $\{1,2,3\}$ :

$$
\begin{gathered}
C_{0}^{3}=\left\{C_{0,1}^{3}\right\}=\operatorname{PSubsets}[\{1,2,3\}, 0]=\{\{\}\} \\
C_{1}^{3}=\left\{C_{1,1}^{3}, C_{1,2}^{3}, C_{1,3}^{3}\right\}=\operatorname{PSubsets}[\{1,2,3\}, 1]=\{\{1\},\{2\},\{3\}\} \\
C_{2}^{3}=\left\{C_{2,1}^{3}, C_{2,2}^{3}, C_{2,3}^{3}\right\}=\operatorname{PSubsets}[\{1,2,3\}, 2]=\{\{1,2\},\{1,3\},\{2,3\}\} \\
C_{3}^{3}=\left\{C_{3,1}^{3}\right\}=\operatorname{PSubsets}[\{1,2,3\}, 0]=\{\{1,2,3\}\}
\end{gathered}
$$

Expandindo cada um dos somatórios da Expressão (A.147):

$$
\sum_{l=1}^{1}\left[\left(\prod_{j \in C_{0, l}^{3}} a_{j}\right)\left(\prod_{j \in C_{3,2-l}^{3}} b_{j}\right)\right]=\left(\prod_{j \in C_{0,1}^{3}} a_{j}\right)\left(\prod_{j \in C_{3,1}^{3}} b_{j}\right)=b_{1} b_{2} b_{3} .
$$




$$
\begin{gathered}
\sum_{l=1}^{3}\left[\left(\prod_{j \in C_{1, l}^{3}} a_{j}\right)\left(\prod_{j \in C_{2,4-l}^{3}} b_{j}\right)\right]= \\
\left(\prod_{j \in C_{1,1}^{3}} a_{j}\right)\left(\prod_{j \in C_{2,3}^{3}} b_{j}\right)+\left(\prod_{j \in C_{1,2}^{3}} a_{j}\right) \\
\left(\prod_{j \in C_{2,2}^{3}} b_{j}\right)+\left(\prod_{j \in C_{1,3}^{3} b_{2} b_{3}+a_{2} b_{1} b_{3}+a_{3} b_{1} b_{2} . \quad(\mathrm{A} .149)} a_{j \in C_{2,1}^{3}} b_{j}\right)= \\
\left(\prod_{l=1}^{3}\left[\left(\prod_{j \in C_{2, l}^{3}} a_{j} a_{j}\right)\left(\prod_{j \in C_{1,4-l}^{3}} b_{j}\right)\right]=\right. \\
\left.\sum_{j \in C_{1,3}^{3}} b_{j}\right)+\left(\prod_{j \in C_{2,2}^{3}} a_{j}\right)\left(\prod_{j \in C_{1,2}^{3}} b_{j}\right)+\left(\prod_{j \in C_{2,3}^{3}} a_{j}\right)\left(\prod_{j \in C_{1,1}^{3}} b_{j}\right)= \\
a_{1} a_{2} b_{3}+a_{1} a_{3} b_{2}+a_{2} a_{3} b_{1} . \quad(\mathrm{A} .150) \\
\sum_{l=1}^{1}\left[\left(\prod_{j \in C_{3, l}^{3}} a_{j}\right)\left(\prod_{j \in C_{0,2-l}^{3}} b_{j}\right)\right]=\left(\prod_{j \in C_{3,1}^{3}} a_{j}\right)\left(\prod_{j \in C_{0,1}^{3}} b_{j}\right)=a_{1} a_{2} a_{3} .
\end{gathered}
$$

Juntando os termos acima, a Expressão (A.147) se torna:

$$
\sum_{p=0}^{3} \sum_{l=1}^{\left(\begin{array}{l}
3 \\
p
\end{array}\right)}\left(a^{3-p} \prod_{j \in C_{p, l}^{n}} b_{j}\right)=
$$

$a_{1} a_{2} a_{3}+a_{1} a_{2} b_{3}+a_{1} a_{3} b_{2}+a_{2} a_{3} b_{1}+a_{1} b_{2} b_{3}+a_{2} b_{1} b_{3}+a_{3} b_{1} b_{2}+b_{1} b_{2} b_{3}=\prod_{j=1}^{3}\left(a_{j}+b_{j}\right)$,

que é exatamente igual ao resultado da Expressão (A.145).

Lema A.7. Sejam $\Delta X(\tau+1)$ e $k(\tau)$ processos tais como definidos no Lema A.5. O processo $m_{\tau+1, \tau}^{P \rightarrow Q}$ definido por:

$$
m_{\tau+1, \tau}^{P \rightarrow Q}=\frac{\left(k(\tau+1)-\frac{E_{\tau}^{P}\{k(\tau+1) \Delta X(\tau+1)\}}{E_{\tau}^{P}\left\{k(\tau+1)(\Delta X(\tau+1))^{2}\right\}} k(\tau+1) \Delta X(\tau+1)\right)}{\left(E_{\tau}^{P}\{k(\tau+1)\}-\frac{\left(E_{\tau}^{P}\{k(\tau+1) \Delta X(\tau+1)\}\right)^{2}}{E_{\tau}^{P}\left\{k(\tau+1)(\Delta X(\tau+1))^{2}\right\}}\right)}
$$

pode ser expresso como:

$$
m_{\tau+1, \tau}^{P \rightarrow Q}=a_{0}\left(\Delta t_{\tau}\right)+a_{1}\left(\Delta t_{\tau}\right) Z_{1}\left(\Delta t_{\tau}\right) Z_{2}\left(\Delta t_{\tau}\right)
$$

onde:

$$
a_{0}\left(\Delta t_{\tau}\right)=\frac{e^{\Delta t_{\tau}\left(r+2 e^{\frac{1}{2} \sigma_{Y}^{2}+\beta_{Y}} \lambda^{P}-\lambda^{P}-\mu\right)}-e^{\Delta t_{\tau}\left(\sigma^{2}+e^{2\left(\sigma_{Y}^{2}+\beta_{Y}\right)} \lambda^{P}\right)}}{e^{\left(-1+2 e^{\frac{1}{2} \sigma_{Y}^{2}+\beta_{Y}}\right) \Delta t_{\tau} \lambda^{P}}-e^{\Delta t_{\tau}\left(\sigma^{2}+e^{2\left(\sigma_{Y}^{2}+\beta_{Y}\right)} \lambda^{P}\right)}},
$$




$$
\begin{gathered}
a_{1}\left(\Delta t_{\tau}\right)=\frac{e^{\left(-1+2 e^{\frac{1}{2} \sigma_{Y}^{2}+\beta_{Y}}\right) \Delta t_{\tau} \lambda^{P}}-e^{\Delta t_{\tau}\left(r+2 e^{\frac{1}{2} \sigma_{Y}^{2}+\beta_{Y}} \lambda^{P}-\lambda^{P}-\mu\right)}}{e^{\left(-1+2 e^{\frac{1}{2} \sigma_{Y}^{2}+\beta_{Y}}\right) \Delta t_{\tau} \lambda^{P}}-e^{\Delta t_{\tau}\left(\sigma^{2}+e^{\left.2\left(\sigma_{Y}^{2}+\beta_{Y}\right)_{\lambda}\right)}\right.},} \\
Z_{1}\left(\Delta t_{\tau}\right)=\exp \left\{\sigma \Delta W^{P}(\tau+1)-\frac{1}{2} \sigma^{2} \Delta t_{\tau}\right\} \\
Z_{2}\left(\Delta t_{\tau}\right)=e^{\left(\lambda^{P}-\lambda^{Q}\right) \Delta t_{\tau}} \prod_{i=1}^{N^{P}\left(\Delta t_{\tau}\right)} \frac{\lambda^{Q} f^{Q}\left(Y_{i}^{P}\right)}{\lambda^{P} f^{P}\left(Y_{i}^{P}\right)} \\
\lambda^{Q}=\lambda^{P} e^{\frac{1}{2} \sigma_{Y}^{2}+\beta_{Y}}, \\
f^{P}\left(Y_{i}^{P}\right)=\frac{1}{\sqrt{2 \pi} \sigma_{Y}} e^{-\frac{1}{2}\left(\frac{Y_{i}^{P}-\beta_{Y}}{\sigma_{Y}}\right)^{2}} \\
f^{Q}\left(Y_{i}^{P}\right)=\frac{1}{\sqrt{2 \pi} \sigma_{Y}} e^{-\frac{1}{2}\left(\frac{Y_{i}^{P}-\left(\sigma_{Y}^{2}+\beta_{Y}\right)}{\sigma_{Y}}\right)^{2}} .
\end{gathered}
$$

Além disso, $E_{\tau}^{P}\left\{m_{\tau+1, \tau}^{P \rightarrow Q}\right\}=1$ e $m_{\tau+1, \tau}^{P \rightarrow Q}$ é $\mathcal{F}_{\tau+1}$-mensurável.

Demonstração. $k(\tau+1)$ tal como definido no Lema A.5 é $\mathcal{F}_{\tau}$-mensurável. Portanto, $m_{\tau+1, \tau}^{P \rightarrow Q}$ (Expressão (A.153)), pode ser rescrito como:

$$
m_{\tau+1, \tau}^{P \rightarrow Q}=\frac{\left(1-\frac{E_{\tau}^{P}\{\Delta X(\tau+1)\}}{E_{\tau}^{P}\left\{(\Delta X(\tau+1))^{2}\right\}} \Delta X(\tau+1)\right)}{\left(1-\frac{\left(E_{\tau}^{P}\{\Delta X(\tau+1)\}\right)^{2}}{E_{\tau}^{P}\left\{(\Delta X(\tau+1))^{2}\right\}}\right)} .
$$

Substituindo na expressão de $m_{\tau+1, \tau}^{P \rightarrow Q}$ acima os resultados do Lema A.4 para $E_{\tau}^{P}\{\Delta X(\tau+1)\}$ e para $E_{\tau}^{P}\left\{(\Delta X(\tau+1))^{2}\right\}$, e a Equação (4.30) para $\Delta X(\tau+1)$, obtemos:

$$
\begin{aligned}
& m_{\tau+1, \tau}^{P \rightarrow Q}= \\
& \frac{1-\frac{\left(e^{(\mu-r) \Delta t_{\tau}}-1\right)\left\{\exp \left[\sigma \Delta W^{P}(\tau+1)+\left(\mu-r-\left(e^{\beta_{Y}+\frac{1}{2} \sigma_{Y}^{2}}-1\right) \lambda^{P}-\frac{1}{2} \sigma^{2}\right) \Delta t_{\tau}\right] \prod_{i=1}^{N^{P}\left(\Delta t_{\tau}\right)} e^{Y_{i}^{P}}-1\right\}}{\exp \left[\left(2 \mu-2 r+\sigma^{2}+\lambda^{P}\left(1-2 e^{\left.\beta_{Y}+\frac{1}{2} \sigma_{Y}^{2}+e^{2\left(\sigma_{Y}^{2}+\beta_{Y}\right.}\right)}\right)\right) \Delta t_{\tau}\right]-2 \exp \left[(\mu-r) \Delta t_{\tau}\right]+1}}{1-\frac{\left(e^{(\mu-r) \Delta t_{\tau}}-1\right)^{2}}{\exp \left[\left(2 \mu-2 r+\sigma^{2}+\lambda^{P}\left(1-2 e^{\beta_{Y}+\frac{1}{2} \sigma_{Y}^{2}}+e^{2\left(\sigma_{Y}^{2}+\beta_{Y}\right.}\right)\right) \Delta t_{\tau}\right]-2 \exp \left[(\mu-r) \Delta t_{\tau}\right]+1}} .
\end{aligned}
$$

Definindo:

$$
a_{0}\left(\Delta t_{\tau}\right)=\frac{e^{\Delta t_{\tau}\left(r+2 e^{\frac{1}{2} \sigma_{Y}^{2}+\beta_{Y}} \lambda^{P}-\lambda^{P}-\mu\right)}-e^{\Delta t_{\tau}\left(\sigma^{2}+e^{2\left(\sigma_{Y}^{2}+\beta_{Y}\right)} \lambda^{P}\right)}}{e^{\left(-1+2 e^{\frac{1}{2} \sigma_{Y}^{2}+\beta_{Y}}\right) \Delta t_{\tau} \lambda^{P}}-e^{\Delta t_{\tau}\left(\sigma^{2}+e^{2\left(\sigma_{Y}^{2}+\beta_{Y}\right)} \lambda^{P}\right)}},
$$




$$
a_{1}\left(\Delta t_{\tau}\right)=\frac{e^{\left(-1+2 e^{\frac{1}{2} \sigma_{Y}^{2}+\beta_{Y}}\right) \Delta t_{\tau} \lambda^{P}}-e^{\Delta t_{\tau}\left(r+2 e^{\frac{1}{2} \sigma_{Y}^{2}+\beta_{Y}} \lambda^{P}-\lambda^{P}-\mu\right)}}{e^{\left(-1+2 e^{\frac{1}{2} \sigma_{Y}^{2}+\beta_{Y}}\right) \Delta t_{\tau} \lambda^{P}}-e^{\Delta t_{\tau}\left(\sigma^{2}+e^{2\left(\sigma_{Y}^{2}+\beta_{Y}\right)} \lambda^{P}\right)}},
$$

a Expressão (A.163) pode ser simplificada para:

$$
\begin{aligned}
m_{\tau+1, \tau}^{P \rightarrow Q}=a_{0}\left(\Delta t_{\tau}\right)+ & a_{1}\left(\Delta t_{\tau}\right) \underbrace{\exp \left(\sigma \Delta W^{P}(\tau+1)-\frac{1}{2} \sigma^{2} \Delta t_{\tau}\right)}_{Z_{1}\left(\Delta t_{\tau}\right)} \\
& \underbrace{\exp \left(\left(1-e^{\beta_{Y}+\frac{1}{2} \sigma_{Y}^{2}}\right) \lambda^{P} \Delta t_{\tau}\right)\left(\prod_{i=1}^{N^{P}\left(\Delta t_{\tau}\right)} e^{Y_{i}^{P}}\right)}_{Z_{2}\left(\Delta t_{\tau}\right)} .
\end{aligned}
$$

Conforme a demonstração do Lema $4.1, Z_{2}\left(\Delta t_{\tau}\right)$ pode ser escrito como:

$$
Z_{2}\left(\Delta t_{\tau}\right)=e^{\left(\lambda^{P}-\lambda^{Q}\right) \Delta t_{\tau}} \prod_{i=1}^{N^{P}\left(\Delta t_{\tau}\right)} \frac{\lambda^{Q} f^{Q}\left(Y_{i}^{P}\right)}{\lambda^{P} f^{P}\left(Y_{i}^{P}\right)}
$$

com $f^{P}\left(Y_{i}^{P}\right), \lambda^{Q}$ e $f^{Q}\left(Y_{i}^{P}\right)$ dados, respectivamente, por (A.100), (A.102), e (A.103). Portanto, $m_{\tau+1, \tau}^{P \rightarrow Q}$ pode ser finalmente escrito como:

$$
m_{\tau+1, \tau}^{P \rightarrow Q}=a_{0}\left(\Delta t_{\tau}\right)+a_{1}\left(\Delta t_{\tau}\right) Z_{1}\left(\Delta t_{\tau}\right) Z_{2}\left(\Delta t_{\tau}\right) .
$$

Além disso, notemos que $m_{\tau+1, \tau}^{P \rightarrow Q}$ é $\mathcal{F}_{\tau+1}$-mensurável, e que $E_{\tau}^{P}\left\{m_{\tau+1, \tau}^{P \rightarrow Q}\right\}=1$. De fato:

$$
\begin{gathered}
E_{\tau}^{P}\left\{m_{\tau+1, \tau}^{P \rightarrow Q}\right\}=E_{\tau}^{P}\left\{a_{0}\left(\Delta t_{\tau}\right)+a_{1}\left(\Delta t_{\tau}\right) Z_{1}\left(\Delta t_{\tau}\right) Z_{2}\left(\Delta t_{\tau}\right)\right\}= \\
a_{0}\left(\Delta t_{\tau}\right)+a_{1}\left(\Delta t_{\tau}\right) E_{\tau}^{P}\left\{Z_{1}\left(\Delta t_{\tau}\right) Z_{2}\left(\Delta t_{\tau}\right)\right\}= \\
a_{0}\left(\Delta t_{\tau}\right)+a_{1}\left(\Delta t_{\tau}\right) E_{\tau}^{P}\left\{Z_{1}\left(\Delta t_{\tau}\right)\right\} E_{\tau}^{P}\left\{Z_{2}\left(\Delta t_{\tau}\right)\right\}=a_{0}\left(\Delta t_{\tau}\right)+a_{1}\left(\Delta t_{\tau}\right)=1 .
\end{gathered}
$$

Portanto, como já era de se esperar conforme os conceitos apresentados no Item (3.1.4), a expressão $m_{\tau+1, \tau}^{P \rightarrow Q}$ pode ser interpretada como uma mudança de medida de probabilidade da medida $P$ para a medida $Q$ (i.e., a derivada de Radon-Nikodým, $\frac{\mathrm{dQ}}{\mathrm{dP}}$,da medida $Q$ com relação à medida $P$ ). E assim completamos a prova.

Lema A.8. Sejam $m_{j+1, j}^{P \rightarrow Q}$ e $m_{\mathcal{T}, \tau}^{P \rightarrow Q}$ tais como definidos, respectivamente, no Lema A.7 e no Corolário 3.10. Admitamos que os $\Delta t_{j}, 0 \leq j \leq \mathcal{T}-1$, possuam a mesma magnitude, i.e., $\Delta t_{j}=\Delta t$. Nestas condições, $m_{\mathcal{T}, \tau}^{P \rightarrow \bar{Q}}$ pode ser escrito como:

$$
m_{\mathcal{T}, \tau}^{P \rightarrow Q}=\sum_{p=0}^{n} a_{0}^{n-p} a_{1}^{p} \sum_{l=1}^{\left(\begin{array}{c}
n \\
p
\end{array}\right)} m_{\mathcal{T}, \tau}^{P \rightarrow Q_{l}}
$$


onde:

$$
\begin{aligned}
& n=\mathcal{T}-\tau, \\
& m_{\mathcal{T}, \tau}^{P \rightarrow Q_{l}}=Z_{1, l}(p \Delta t) Z_{2}(p \Delta t), \\
& Z_{1, l}(p \Delta t)=\exp \left(\sum_{j \in C_{p, l}^{n}}\left(\sigma \Delta W^{P}(\tau+j)-\frac{1}{2} \sigma^{2} \Delta t\right)\right)= \\
& \exp \left(\sum_{j=1}^{n}\left(\sigma_{l}(\tau+j-1) \Delta W^{P}(\tau+j)-\frac{1}{2} \sigma_{l}^{2}(\tau+j-1) \Delta t\right)\right), \\
& \sigma_{l}(\tau+j-1)=\sigma \mathbf{I}_{C_{p, l}^{n}}(j), \\
& \mathbf{I}_{C_{p, l}^{n}}(j)=\left\{\begin{array}{lll}
1, & \text { se } & j \in C_{p, l}^{n} \\
0, & \text { se } & j \notin C_{p, l}^{n}
\end{array},\right. \\
& Z_{2}(p \Delta t)=e^{\left(\lambda^{P}-\lambda^{Q}\right) p \Delta t} \prod_{i=1}^{N^{P}(p \Delta t)} \frac{\lambda^{Q} f^{Q}\left(Y_{i}^{P}\right)}{\lambda^{P} f^{P}\left(Y_{i}^{P}\right)} \\
& \lambda^{Q}=\lambda^{P} e^{\frac{1}{2} \sigma_{Y}^{2}+\beta_{Y}}, \\
& f^{P}\left(Y_{i}^{P}\right)=\frac{1}{\sqrt{2 \pi} \sigma_{Y}} e^{-\frac{1}{2}\left(\frac{Y_{i}^{P}-\beta_{Y}}{\sigma_{Y}}\right)^{2}}, \\
& f^{Q}\left(Y_{i}^{P}\right)=\frac{1}{\sqrt{2 \pi} \sigma_{Y}} e^{-\frac{1}{2}\left(\frac{Y_{i}^{P}-\left(\sigma_{Y}^{2}+\beta_{Y}\right)}{\sigma_{Y}}\right)^{2}} .
\end{aligned}
$$

Demonstraçãoo. Como se pode verificar facilmente pelas Equações (A.155) e (A.156), a hipótese de que $\Delta t_{j}=\Delta t$ faz com que $a_{0}\left(\Delta t_{j}\right)$ e $a_{1}\left(\Delta t_{j}\right)$ sejam constantes. Portanto, passemos a nos referir a estas variáveis simplesmente como $a_{0}$ e $a_{1}$.

Apesar de os $\Delta t_{j}, 0 \leq j \leq \mathcal{T}-1$, possuírem a mesma magnitude, manteremos os índices ao longo desta prova para evitar confusão na manipulação das variáveis aleatórias $N^{P}\left(\Delta t_{\tau+j-1}\right)$. Logo que apropriado, eliminamos os índices.

Escrevamos a Expressão (A.154) do Lema A.7 para dois instantes de tempo consecutivos quaisquer $\tau+j-1$ e $\tau+j, 1 \leq j \leq \mathcal{T}-\tau$ :

$$
m_{\tau+j, \tau+j-1}^{P \rightarrow Q}=a_{0}+a_{1} Z_{1}\left(\Delta t_{\tau+j-1}\right) Z_{2}\left(\Delta t_{\tau+j-1}\right),
$$

onde:

$$
Z_{1}\left(\Delta t_{\tau+j-1}\right)=\exp \left\{\sigma \Delta W^{P}(\tau+j)-\frac{1}{2} \sigma^{2} \Delta t_{\tau+j-1}\right\}
$$




$$
Z_{2}\left(\Delta t_{\tau+j-1}\right)=e^{\left(\lambda^{P}-\lambda^{Q}\right) \Delta t_{\tau+j-1}} \prod_{i=1}^{N^{P}\left(\Delta t_{\tau+j-1}\right)} \frac{\lambda^{Q} f^{Q}\left(Y_{i}^{P}\right)}{\lambda^{P} f^{P}\left(Y_{i}^{P}\right)} .
$$

Substituindo esta expressão na expressão de $m_{\mathcal{T}, \tau}^{P \rightarrow Q}$ (Equação 3.66) do Corolário 3.10, obtemos:

$$
\begin{aligned}
m_{\mathcal{T}, \tau}^{P \rightarrow Q}=\prod_{j=\tau}^{\mathcal{T}-1} m_{j+1, j}^{P \rightarrow Q}= & \prod_{j=1}^{n=\mathcal{T}-\tau} m_{\tau+j, \tau+j-1}^{P \rightarrow Q}= \\
& \prod_{j=1}^{n=\mathcal{T}-\tau}(\underbrace{a_{0}}_{b_{j}}+\underbrace{a_{1} Z_{1}\left(\Delta t_{\tau+j-1}\right) Z_{2}\left(\Delta t_{\tau+j-1}\right)}_{a_{j}}) .
\end{aligned}
$$

Usando o Lema A.6, a expressão acima pode ser rescrita como:

$$
m_{\mathcal{T}, \tau}^{P \rightarrow Q}=\prod_{j=1}^{n}\left(b_{j}+a_{j}\right)=\sum_{p=0}^{n} \sum_{l=1}^{\left(\begin{array}{l}
n \\
p
\end{array}\right)}\left(a_{0}^{n-p} \prod_{j \in C_{p, l}^{n}}\left[a_{1} Z_{1}\left(\Delta t_{\tau+j-1}\right) Z_{2}\left(\Delta t_{\tau+j-1}\right)\right]\right) .
$$

Expandindo o produtório em $j \in C_{p, l}^{n}$, a expressão acima se torna:

$$
m_{\mathcal{T}, \tau}^{P \rightarrow Q}=\sum_{p=0}^{n} \sum_{l=1}^{\left(\begin{array}{c}
n \\
p
\end{array}\right)}(a_{0}^{n-p} a_{1}^{p} \underbrace{\prod_{j \in C_{p, l}^{n}} Z_{1}\left(\Delta t_{\tau+j-1}\right)}_{Z_{1, l}(p \Delta t)} \underbrace{\prod_{j \in C_{p, l}^{n}} Z_{2}\left(\Delta t_{\tau+j-1}\right)}_{Z_{2}(p \Delta t)}) .
$$

O termo $Z_{1, l}(p \Delta t)$ pode ser escrito como:

$$
\begin{gathered}
Z_{1, l}(p \Delta t)=\prod_{j \in C_{p, l}^{n}} Z_{1}\left(\Delta t_{\tau+j-1}\right)=\prod_{j \in C_{p, l}^{n}} \exp \left(\sigma \Delta W^{P}(\tau+j)-\frac{1}{2} \sigma^{2} \Delta t_{\tau+j-1}\right)= \\
\quad \exp \left(\sum_{j \in C_{p, l}^{n}}\left(\sigma \Delta W^{P}(\tau+j)-\frac{1}{2} \sigma^{2} \Delta t\right)\right)= \\
\exp \left(\sum_{j=1}^{n}(\underbrace{\sigma \mathbf{I}_{C_{p, l}^{n}}(j)}_{\sigma_{l}(\tau+j-1)} \Delta W^{P}(\tau+j)-\frac{1}{2} \underbrace{\left(\sigma \mathbf{I}_{C_{p, l}^{n}}(j)\right)^{2}}_{\sigma_{l}^{2}(\tau+j-1)} \Delta t), \quad\right. \text { (A.186) }
\end{gathered}
$$

onde $\mathbf{I}_{A}(x)$ é a função indicadora de $x$ com relação ao conjunto $A$, i.e., $\mathbf{I}_{A}(x)=1$ se $x \in A$, e $\mathbf{I}_{A}(x)=0$ se $x \notin A$. 
O termo $Z_{2}(p \Delta t)$ pode ser escrito como:

$$
\begin{gathered}
Z_{2}(p \Delta t)=\prod_{j \in C_{p, l}^{n}} Z_{2}\left(\Delta t_{\tau+j-1}\right)= \\
\prod_{j \in C_{p, l}^{n}}\left(e^{\left(\lambda^{P}-\lambda^{Q}\right) \Delta t_{\tau+j-1}} \prod_{i=1}^{N^{P}\left(\Delta t_{\tau+j-1}\right)} \frac{\lambda^{Q} f^{Q}\left(Y_{i}^{P}\right)}{\lambda^{P} f^{P}\left(Y_{i}^{P}\right)}\right)= \\
\underbrace{\left(\prod_{j \in C_{p, l}^{n}} e^{\left(\lambda^{P}-\lambda^{Q}\right) \Delta t_{\tau+j-1}}\right)}_{Z_{2, a}(p \Delta t)} \underbrace{\left[\prod_{j \in C_{p, l}^{n}}\left(\prod_{i=1}^{N^{P}\left(\Delta t_{\tau+j-1}\right)} \frac{\lambda^{Q} f^{Q}\left(Y_{i}^{P}\right)}{\lambda^{P} f^{P}\left(Y_{i}^{P}\right)}\right)\right]}_{Z_{2, b}(p \Delta t)} .
\end{gathered}
$$

O termo $Z_{2, a}(p \Delta t)$ pode ser simplificado para:

$$
Z_{2, a}(p \Delta t)=e^{\left(\lambda^{P}-\lambda^{Q}\right) p \Delta t} .
$$

O termo $Z_{2, b}(p \Delta t)$ pode ser simplificado para:

$$
\begin{aligned}
Z_{2, b}(p \Delta t)=\prod_{i=1}^{\sum_{j \in C_{p, l}^{n}} \prod^{N^{P}\left(\Delta t_{\tau+j-1}\right)} \frac{\lambda^{Q} f^{Q}\left(Y_{i}^{P}\right)}{\lambda^{P} f^{P}\left(Y_{i}^{P}\right)}}= \\
\prod_{i=1}^{N^{P}\left(\sum_{\left.j \in C_{p, l}^{n} \Delta t_{\tau+j-1}\right)} \frac{\lambda^{Q} f^{Q}\left(Y_{i}^{P}\right)}{\lambda^{P} f^{P}\left(Y_{i}^{P}\right)}\right.}=\prod_{i=1}^{N^{P}(p \Delta t)} \frac{\lambda^{Q} f^{Q}\left(Y_{i}^{P}\right)}{\lambda^{P} f^{P}\left(Y_{i}^{P}\right)} .
\end{aligned}
$$

Definindo:

$$
m_{\mathcal{T}, \tau}^{P \rightarrow Q_{l}}=Z_{1, l}(p \Delta t) Z_{2}(p \Delta t)=Z_{1, l}(p \Delta t) \underbrace{Z_{2, a}(p \Delta t) Z_{2, b}(p \Delta t)}_{Z_{2}(p \Delta t)},
$$

podemos escrever $m_{\mathcal{T}, \tau}^{P \rightarrow Q}$ na Expressão (A.185) da seguinte forma:

$$
m_{\mathcal{T}, \tau}^{P \rightarrow Q}=\sum_{p=0}^{n} \sum_{l=1}^{\left(\begin{array}{l}
n \\
p
\end{array}\right)} a_{0}^{n-p} a_{1}^{p} m_{\mathcal{T}, \tau}^{P \rightarrow Q_{l}}=\sum_{p=0}^{n} a_{0}^{n-p} a_{1}^{p} \sum_{l=1}^{\left(\begin{array}{l}
n \\
p
\end{array}\right)} m_{\mathcal{T}, \tau}^{P \rightarrow Q_{l}}
$$

o que completa a prova.

Demonstração do Teorema 4.2. Substituindo a expressão de $m_{\mathcal{T}, \tau}^{P \rightarrow Q}$ (Lema A.8), Equação (A.170) na expressão de $H(\tau)$ (Corolário 3.10, Equação (3.65)), obtemos:

$$
\begin{gathered}
H(\tau)=E_{\tau}^{P}\left\{\sum_{p=0}^{n} a_{0}^{n-p} a_{1}^{p} \sum_{l=1}^{\left(\begin{array}{l}
n \\
p
\end{array}\right)} m_{\mathcal{T}, \tau}^{P \rightarrow Q_{l}} \frac{H(\mathcal{T})}{\prod_{j=\tau}^{\mathcal{T}-1} a(j)}\right\}= \\
E_{\tau}^{P}\left\{\sum_{p=0}^{n} a_{0}^{n-p} a_{1}^{p} \sum_{l=1}^{\left(\begin{array}{l}
n \\
p
\end{array}\right)}\left(m_{\mathcal{T}, \tau}^{P \rightarrow Q_{l}} \frac{H(\mathcal{T})}{\prod_{j=\tau}^{\mathcal{T}-1} a(j)}\right)\right\} .
\end{gathered}
$$


Como a esperança é um operador linear, podemos passar o operador $E_{\tau}^{P}\{\cdot\}$ para dentro dos somatórios, obtendo:

$$
H(\tau)=\sum_{p=0}^{n}\left(a_{0}^{n-p} a_{1}^{p} \sum_{l=1}^{\left(\begin{array}{l}
n \\
p
\end{array}\right)} E_{\tau}^{P}\left\{m_{\mathcal{T}, \tau}^{P \rightarrow Q_{l}} \frac{H(\mathcal{T})}{\prod_{j=\tau}^{\mathcal{T}-1} a(j)}\right\}\right) .
$$

O produtório $\prod_{j=\tau}^{\mathcal{T}-1} a(j)$ pode ser rescrito como:

$$
\prod_{j=\tau}^{\mathcal{T}-1} a(j)=\prod_{j=\tau}^{\mathcal{T}-1}(1+r(j))=\frac{S_{0}(\mathcal{T})}{S_{0}(\tau)}=e^{r(\mathcal{T}-\tau) \Delta t}=e^{r n \Delta t} .
$$

Portanto, a Equação (A.193) se torna:

$$
H(\tau)=e^{-r n \Delta t} \sum_{p=0}^{n}\left(a_{0}^{n-p} a_{1}^{p} \sum_{l=1}^{\left(\begin{array}{c}
n \\
p
\end{array}\right)} E_{\tau}^{P}\left\{m_{\mathcal{T}, \tau}^{P \rightarrow Q_{l}} H(\mathcal{T})\right\}\right) .
$$

Conforme mostrado em [32], $m_{\mathcal{T}, \tau}^{P \rightarrow Q_{l}}$, tal como definido no Lema (A.172), é a mudança de medida de probabilidade da medida $P$ para a medida $Q^{l}$, o que nos permite escrever a Equação (A.195) como:

$$
H(\tau)=e^{-r n \Delta t} \sum_{p=0}^{n}\left(a_{0}^{n-p} a_{1}^{p} \sum_{l=1}^{\left(\begin{array}{c}
n \\
p
\end{array}\right)} E_{\tau}^{Q_{l}}\{H(\mathcal{T})\}\right) .
$$

Devido a esta mudança de medida de probabilidade, quando do cálculo de $E_{\tau}^{Q_{l}}\{\cdot\}$, as variáveis aleatórias $\Delta W^{P}(\tau+j)$ são substituídas por $\Delta W^{Q_{l}}(\tau+j)$, que permanecem independentes e identicamente distribuídas sob a nova medida $Q_{l}$, respeitando-se as seguintes relações (dadas pelo teorema de Girsanov):

$$
\begin{gathered}
\Delta W^{P}(\tau+j)=\Delta W^{Q_{l}}(\tau+j)+\sigma_{l}(\tau+j-1) \Delta t, \\
\Delta W^{P}(\tau+j) N(0, \Delta t) \text { com respeito à medida } P, \\
\Delta W^{P}(\tau+j) N\left(\sigma_{l}(\tau+j-1) \Delta t, \Delta t\right) \text { com respeito à medida } Q_{l}, \\
\Delta W^{Q_{l}}(\tau+j) N(0, \Delta t) \text { com respeito à medida } Q_{l} .
\end{gathered}
$$

As variáveis aleatórias $Y_{i}^{P}$ são substituídas por $Y_{i}^{Q}$, que permanecem independentes e identicamente distribuídas sob a nova medida $Q_{l}$, respeitando-se as seguintes relações:

$$
\begin{gathered}
Y_{i}^{P}=Y_{i}^{Q}+\sigma_{Y}^{2}, \\
Y_{i}^{P} \sim N\left(\beta_{Y}, \sigma_{Y}^{2}\right) \text { com respeito à medida } P, \\
Y_{i}^{P} \sim N\left(\sigma_{Y}^{2}+\beta_{Y}, \sigma_{Y}^{2}\right) \text { com respeito à medida } Q_{l}, \\
Y_{i}^{Q} \sim N\left(\beta_{Y}, \sigma_{Y}^{2}\right) \text { com respeito à medida } Q_{l} .
\end{gathered}
$$

Além disso, o processo de Poisson $N^{P}(\cdot)$, de intensidade $\lambda^{P}$ com relação à medida de probabilidade $P$, é substituído por um novo processo de Poisson 
$N^{Q}(\cdot)$, de intensidade $\lambda^{Q}=\lambda^{P} e^{\frac{1}{2} \sigma_{Y}^{2}+\beta_{Y}}$ com relação à medida de probabilidade $Q_{l}$.

Demonstração do Teorema 4.3. Expandindo o numerador da expressão de $\bar{u}(\tau)$, obtemos:

$$
\bar{u}(\tau)=\frac{E_{\tau}^{P}\left\{k(\tau+1) \Delta X(\tau+1) \frac{H(\tau+1)}{S_{0}(\tau+1)}\right\}-E_{\tau}^{P}\left\{k(\tau+1) \Delta X(\tau+1) \frac{V(\tau)}{S_{0}(\tau)}\right\}}{E_{\tau}^{P}\left\{k(\tau+1)(\Delta X(\tau+1))^{2}\right\}} .
$$

Usando o fato de que $V(\tau), S_{0}(\tau), S_{0}(\tau+1)$ e $k(\tau+1)$ são $\mathcal{F}_{\tau}$-mensuráveis, e que $S_{0}(\tau+1)=S_{0}(\tau) a(\tau)$, temos:

$$
\bar{u}(\tau)=\frac{\overbrace{E_{\tau}^{P}\left\{\Delta X(\tau+1) \frac{H(\tau+1)}{a(\tau)}\right\}}^{T_{1}}-\overbrace{V(\tau) E_{\tau}^{P}\{\Delta X(\tau+1)\}}^{T_{2}}}{S_{0}(\tau) E_{\tau}^{P}\left\{(\Delta X(\tau+1))^{2}\right\}} .
$$

Sob a hipótese do Lema A.8 de que $\Delta t_{j}=\Delta t, 0 \leq j \leq \mathcal{T}-1$, e usando os resultados do Lema A.4, o termo $T_{2}$ da Expressão (A.198) pode ser escrito como:

$$
T_{2}=V(\tau) \frac{S(\tau)}{S_{0}(\tau)}\left(e^{(\mu-r) \Delta t}-1\right) .
$$

Para calcularmos o termo $T_{1}$ da Expressão (A.198), precisamos obter inicialmente $H(\tau+1)$. Aplicando as Equações (3.65), (3.66) e (A.154) para o instante de tempo discreto $\tau+1$, temos:

$$
H(\tau+1)=E_{\tau+1}^{P}\left\{\prod_{j=1}^{\check{n}}\left(a_{0}+a_{1} Z_{1}\left(\Delta t_{\tau+j}\right) Z_{2}\left(\Delta t_{\tau+j}\right)\right) \frac{H(\mathcal{T})}{\left(\prod_{j=\tau+1}^{\mathcal{T}-1} a(j)\right)}\right\},
$$

onde $\check{n}=\mathcal{T}-(\tau+1)=n-1$.

Lembrando que $a(\tau)=e^{r \Delta t}$, o produtório $\prod_{j=\tau+1}^{\mathcal{T}-1} a(j)$ pode ser rescrito como:

$$
\prod_{j=\tau+1}^{\mathcal{T}-1} a(j)=e^{r(\mathcal{T}-\tau-1) \Delta t}=e^{r \check{n} \Delta t} .
$$

Usando as Equações (A.200), (A.201) e os resultados do Lema 4.1, o termo $T_{1}$ da Expressão (A.198) pode ser escrito como:

$$
\begin{aligned}
E_{\tau}^{P}\left\{\Delta X(\tau+1) \frac{H(\tau+1)}{a(\tau)}\right\}= & \\
& E_{\tau}^{P}\left\{\frac{S(\tau)}{S_{0}(\tau)}\left(e^{(\mu-r) \Delta t}-1\right)\left(\alpha_{0}+\alpha_{1} Z_{1}\left(\Delta t_{\tau}\right) Z_{2}\left(\Delta t_{\tau}\right)\right)\right. \\
& \left.\frac{E_{\tau+1}^{P}\left\{\prod_{j=1}^{\check{n}}\left(a_{0}+a_{1} Z_{1}\left(\Delta t_{\tau+j}\right) Z_{2}\left(\Delta t_{\tau+j}\right)\right) H(\mathcal{T})\right\}}{e^{r \Delta t} e^{r \check{n} \Delta t}}\right\} .
\end{aligned}
$$


O termo $\left[\alpha_{0}+\alpha_{1} Z_{1}\left(\Delta t_{\tau}\right) Z_{2}\left(\Delta t_{\tau}\right)\right]$ é $\mathcal{F}_{\tau+1}$-mensurável. Portanto, ele pode ser passado para dentro da esperança $E_{\tau+1}^{P}\{\cdot\}$. Podemos então rescrever a Expressão (A.202) como:

$$
\begin{aligned}
& E_{\tau}^{P}\left\{\Delta X(\tau+1) \frac{H(\tau+1)}{a(\tau)}\right\}= \\
& \frac{S(\tau)}{S_{0}(\tau)} \frac{\left(e^{(\mu-r) \Delta t}-1\right)}{e^{r n \Delta t}} E_{\tau}^{P}\left\{E _ { \tau + 1 } ^ { P } \left\{\left(\left(\alpha_{0}+\alpha_{1} Z_{1}\left(\Delta t_{\tau}\right) Z_{2}\left(\Delta t_{\tau}\right)\right)\right.\right.\right. \\
&\left.\left.\left.\prod_{j=1}^{\check{n}}\left(a_{0}+a_{1} Z_{1}\left(\Delta t_{\tau+j}\right) Z_{2}\left(\Delta t_{\tau+j}\right)\right)\right) H(\mathcal{T})\right\}\right\} .
\end{aligned}
$$

Usando a lei das expectativas iteradas, $E_{\tau}^{P}\left\{E_{\tau+1}^{P}\{\cdot\}\right\}=E_{\tau}^{P}\{\cdot\}$, esta expressão se torna:

$$
\begin{gathered}
E_{\tau}^{P}\left\{\Delta X(\tau+1) \frac{H(\tau+1)}{a(\tau)}\right\}= \\
\frac{S(\tau)}{S_{0}(\tau)} \frac{\left(e^{(\mu-r) \Delta t}-1\right)}{e^{r n \Delta t}} \\
E_{\tau}^{P}\{\underbrace{\left(\left[\alpha_{0}+\alpha_{1} Z_{1}\left(\Delta t_{\tau}\right) Z_{2}\left(\Delta t_{\tau}\right)\right] \prod_{j=1}^{\check{n}}\left(a_{0}+a_{1} Z_{1}\left(\Delta t_{\tau+j}\right) Z_{2}\left(\Delta t_{\tau+j}\right)\right)\right)}_{m_{\mathcal{T}, \tau}^{P \rightarrow S}} H(\mathcal{T})\} .
\end{gathered}
$$

Definindo:

$$
\begin{aligned}
& A_{0}(j)=\left\{\begin{array}{ll}
a_{0} & j=2, \cdots, \mathcal{T}-\tau \\
\alpha_{0} & j=1
\end{array},\right. \\
& A_{1}(j)=\left\{\begin{array}{ll}
a_{1} & j=2, \cdots, \mathcal{T}-\tau \\
\alpha_{1} & j=1
\end{array},\right.
\end{aligned}
$$

podemos rescrever $m_{\mathcal{T}, \tau}^{P \rightarrow S}$ na Expressão (A.204) da seguinte forma:

$$
m_{\mathcal{T}, \tau}^{P \rightarrow S}=\prod_{j=1}^{n}\left(A_{0}(j)+A_{1}(j) Z_{1}\left(\Delta t_{\tau+j-1}\right) Z_{2}\left(\Delta t_{\tau+j-1}\right)\right) .
$$

Usando agora o resultado do Lema A.6, a Expressão (A.207) pode ser rescrita 
como:

$$
\begin{aligned}
m_{\mathcal{T}, \tau}^{P \rightarrow S}= & \\
\sum_{p=0}^{n} \sum_{l=1}^{\left(\begin{array}{c}
n \\
p
\end{array}\right)}\left[\left(\prod_{j \in C_{p, l}^{n}} A_{1}(j) Z_{1}\left(\Delta t_{\tau+j-1}\right) Z_{2}\left(\Delta t_{\tau+j-1}\right)\right)\right. & \left.\left(\prod_{j \in C_{n-p, s}^{n}} A_{0}(j)\right)\right]= \\
\sum_{p=0}^{n} \sum_{l=1}^{\left.\sum_{p}^{(}\right)}\left[\left(\prod_{j \in C_{p, l}^{n}} A_{1}(j)\right)\left(\prod_{j \in C_{n-p, s}^{n}} A_{0}(j)\right)\right. & \underbrace{\left(\prod_{j \in C_{p, l}^{n}} Z_{1}\left(\Delta t_{\tau+j-1}\right)\right)}_{Z_{1, l}(p \Delta t)} \underbrace{\left.\left(\prod_{j \in C_{p, l}^{n}} Z_{2}\left(\Delta t_{\tau+j-1}\right)\right)\right]}_{Z_{2}(p \Delta t)}, \quad \text { A.2.208) }
\end{aligned}
$$

onde $s=\left[\left(\begin{array}{l}n \\ p\end{array}\right)-l+1\right]$.

Façamos as seguintes simplificações:

$$
\begin{aligned}
& \prod_{j \in C_{p, l}^{n}} A_{1}(j)=a_{1}^{p-1} a_{1}^{\left(1-\mathbf{I}_{C_{p, l}^{n}}(1)\right)} \alpha_{1}^{\mathbf{I}_{C_{p, l}^{n}}(1)}=a_{1}^{p}\left(\frac{\alpha_{1}}{a_{1}}\right)^{\mathbf{I}_{C_{p, l}^{n}}(1)}, \\
& \prod_{j \in C_{n-p, s}^{n}} A_{0}(j)=a_{0}^{n-p-1} a_{0}^{\left(1-\mathbf{I}_{C_{n-p, s}^{n}}(1)\right)} \alpha_{0}^{\mathbf{I}_{C_{p, l}^{n}}(1)}=a_{0}^{n-p}\left(\frac{\alpha_{0}}{a_{0}}\right)^{\mathbf{I}_{C_{n-p, s}^{n}}(1)} .
\end{aligned}
$$

Além disso, como já mostrado anteriormente, $Z_{1, l}(p \Delta t)$ (Equação (A.186)) é dada por:

$$
\begin{aligned}
& Z_{1, l}(p \Delta t)= \prod_{j \in C_{p, l}^{n}} Z_{1}\left(\Delta t_{\tau+j-1}\right)=\prod_{j \in C_{p, l}^{n}} \exp \left(\sigma \Delta W^{P}(\tau+j)-\frac{1}{2} \sigma^{2} \Delta t_{\tau+j-1}\right)= \\
& \exp \left(\sum_{j \in C_{p, l}^{n}}\left(\sigma \Delta W^{P}(\tau+j)-\frac{1}{2} \sigma^{2} \Delta t\right)\right)= \\
& \exp \left(\sum_{j=1}^{n}(\underbrace{\sigma \mathbf{I}_{C_{p, l}^{n}}(j)}_{\sigma_{l}(\tau+j-1)} \Delta W^{P}(\tau+j)-\frac{1}{2} \underbrace{\left(\sigma \mathbf{I}_{C_{p, l}^{n}}(j)\right)^{2}}_{\sigma_{l}^{2}(\tau+j-1)} \Delta t), \quad(\mathrm{A} .211)\right.
\end{aligned}
$$

e $Z_{2}(p \Delta t)$ (Equação (A.187)) é dado por:

$$
\begin{aligned}
Z_{2}(p \Delta t)= & \prod_{j \in C_{p, l}^{n}} Z_{2}\left(\Delta t_{\tau+j-1}\right)= \\
& \prod_{j \in C_{p, l}^{n}}\left(e^{\left(\lambda^{P}-\lambda^{Q}\right) \Delta t_{\tau+j-1}} \prod_{i=1}^{N^{P}\left(\Delta t_{\tau+j-1}\right)} \frac{\lambda^{Q} f^{Q}\left(Y_{i}^{P}\right)}{\lambda^{P} f^{P}\left(Y_{i}^{P}\right)}\right)= \\
& e^{\left(\lambda^{P}-\lambda^{Q}\right) p \Delta t} \prod_{i=1}^{N^{P}(p \Delta t)} \frac{\lambda^{Q} f^{Q}\left(Y_{i}^{P}\right)}{\lambda^{P} f^{P}\left(Y_{i}^{P}\right)} .
\end{aligned}
$$


Portanto, podemos rescrever $m_{\mathcal{T}, \tau}^{P \rightarrow S}$, Equação (A.208), como:

$$
m_{\mathcal{T}, \tau}^{P \rightarrow S}=\sum_{p=0}^{n} a_{1}^{p} a_{0}^{n-p} \sum_{l=1}^{\left(\begin{array}{c}
n \\
p
\end{array}\right)}\left[\left(\frac{\alpha_{1}}{a_{1}}\right)^{\mathbf{I}_{C_{p, l}^{n}}(1)}\left(\frac{\alpha_{0}}{a_{0}}\right)^{\mathbf{I}_{C_{n-p, s}^{n}}(1)} Z_{1, l}(p \Delta t) Z_{2}(p \Delta t)\right] .
$$

Substituindo esse resultado na Equação (A.204), o termo $T_{1}$ da Expressão (A.198) pode ser escrito como:

$$
\begin{gathered}
T_{1}=\frac{S(\tau)}{S_{0}(\tau)} \frac{\left(e^{(\mu-r) \Delta t}-1\right)}{e^{r n \Delta t}} E_{\tau}^{P}\left\{m_{\mathcal{T}, \tau}^{P \rightarrow S} H(\mathcal{T})\right\}= \\
\frac{S(\tau)}{S_{0}(\tau)} \frac{\left(e^{(\mu-r) \Delta t}-1\right)}{e^{r n \Delta t}} \\
E_{\tau}^{P}\left\{\sum_{p=0}^{n} a_{1}^{p} a_{0}^{n-p} \sum_{l=1}^{\left(\begin{array}{c}
n \\
p
\end{array}\right)}\left[\left(\frac{\alpha_{1}}{a_{1}}\right)^{\mathbf{I}_{C_{p, l}^{n}(1)}}\left(\frac{\alpha_{0}}{a_{0}}\right)^{\mathbf{I}_{C_{n-p, s}^{n}}(1)} Z_{1, l}(p \Delta t) Z_{2}(p \Delta t)\right] H(\mathcal{T})\right\} .
\end{gathered}
$$

Como a esperança é um operador linear, obtemos:

$$
\begin{gathered}
T_{1}=\frac{S(\tau)}{S_{0}(\tau)} \frac{\left(e^{(\mu-r) \Delta t}-1\right)}{e^{r n \Delta t}} \\
\left\{\sum_{p=0}^{n} a_{1}^{p} a_{0}^{n-p} \sum_{l=1}^{\left(\begin{array}{l}
n \\
p
\end{array}\right)}\left[\left(\frac{\alpha_{1}}{a_{1}}\right)^{\mathbf{I}_{C_{p, l}^{n}}(1)}\left(\frac{\alpha_{0}}{a_{0}}\right)^{\mathbf{I}_{C_{n-p, s}^{n}}(1)} E_{\tau}^{P}\{\underbrace{Z_{1, l}(p \Delta t) Z_{2}(p \Delta t)}_{m_{\mathcal{T}, \tau}^{P \rightarrow Q_{l}}} H(\mathcal{T})\}\right],\right.
\end{gathered}
$$

onde $m_{\mathcal{T}, \tau}^{P \rightarrow Q_{l}}$ é a mesma mudança de medida já usada na demonstração do Lema A.8, Equação (A.190).

Usando as Expressões (A.215), (A.199) e os resultados do Lema A.4, obtemos $\bar{u}(\tau)$ (Equação (A.198)):

$$
\begin{gathered}
\bar{u}(\tau)=\frac{S(\tau)}{S_{0}(\tau)} \frac{\left(e^{(\mu-r) \Delta t}-1\right)}{e^{r n \Delta t}} \frac{\left(A-V(\tau) \frac{S(\tau)}{S_{0}(\tau)}\left(e^{(\mu-r) \Delta t}-1\right)\right)}{B} \\
A=\sum_{p=0}^{n} a_{1}^{p} a_{0}^{n-p} \sum_{l=1}^{\left(\begin{array}{l}
n \\
p
\end{array}\right)}\left[\left(\frac{\alpha_{1}}{a_{1}}\right)^{\mathbf{I}_{C_{p, l}^{n}}(1)}\left(\frac{\alpha_{0}}{a_{0}}\right)^{\mathbf{I}_{C_{n-p, s}^{n}}^{(1)}} E_{\tau}^{P}\left\{m_{\mathcal{T}, \tau}^{P \rightarrow Q_{l}} H(\mathcal{T})\right\}\right] \\
B=S_{0}(\tau)\left(\frac{S(\tau)}{S_{0}(\tau)}\right)^{2}\left\{\operatorname { e x p } \left[\left(2 \mu-2 r+\sigma^{2}+\right.\right.\right. \\
\left.\left.\left.\quad \lambda^{P}\left(1-2 e^{\beta_{Y}+\frac{1}{2} \sigma_{Y}^{2}}+e^{2\left(\sigma_{Y}^{2}+\beta_{Y}\right)}\right)\right) \Delta t\right]-2 \exp [(\mu-r) \Delta t]+1\right\} .
\end{gathered}
$$


Simplificando, temos:

$$
\begin{gathered}
\bar{u}(\tau)=\left(e^{(\mu-r) \Delta t}-1\right) \frac{A-V(\tau)}{B}, \\
A=\sum_{p=0}^{n} e^{-r n \Delta t} a_{1}^{p} a_{0}^{n-p} \sum_{l=1}^{\left(\begin{array}{c}
n \\
p
\end{array}\right)}\left[\left(\frac{\alpha_{1}}{a_{1}}\right)^{\mathbf{I}_{C_{p, l}^{n}}(1)}\left(\frac{\alpha_{0}}{a_{0}}\right)^{\mathbf{I}_{C_{n-p, s}^{n}}(1)} E_{\tau}^{P}\left\{m_{\mathcal{T}, \tau}^{P \rightarrow Q_{l}} H(\mathcal{T})\right\}\right], \\
B=S(\tau)\left\{\operatorname { e x p } \left[\left(2 \mu-2 r+\sigma^{2}+\right.\right.\right. \\
\left.\left.\left.\lambda^{P}\left(1-2 e^{\beta_{Y}+\frac{1}{2} \sigma_{Y}^{2}}+e^{2\left(\sigma_{Y}^{2}+\beta_{Y}\right)}\right)\right) \Delta t\right]-2 \exp [(\mu-r) \Delta t]+1\right\} .
\end{gathered}
$$

Mudando a medida de probabilidade para a medida $Q_{l}$, temos:

$$
\bar{u}(\tau)=\left(e^{(\mu-r) \Delta t}-1\right) \frac{A-V(\tau)}{B},
$$

onde

$$
A=\sum_{p=0}^{n} e^{-r n \Delta t} a_{1}^{p} a_{0}^{n-p} \sum_{l=1}^{\left(\begin{array}{c}
n \\
p
\end{array}\right)}\left[\left(\frac{\alpha_{1}}{a_{1}}\right)^{\mathbf{I}_{C_{p, l}^{n}}(1)}\left(\frac{\alpha_{0}}{a_{0}}\right)^{\mathbf{I}_{C_{n-p, s}^{n}}(1)} E_{\tau}^{Q_{l}}\{H(\mathcal{T})\}\right],
$$

e $B$ é dado pela Equação (A.305).

Como último passo para completar a prova, devemos obter a equação do valor do portfólio, $V(\tau)$, para o modelo em tempo discreto, com espaço de estados contínuo, com 1 ativo de risco e sem cadeia de Markov. Substituindo $\theta(\tau)=1$ na Equação (3.8), e lembrando que:

$$
\begin{aligned}
S^{P}(\tau+1)+\delta(\tau+1) & =S^{P}(\tau+1) e^{\rho \Delta t}, \\
(1+r(\tau)) & =e^{r \Delta t},
\end{aligned}
$$

obtemos:

$$
V(\tau+1)=e^{r \Delta t} V(\tau)+u(\tau)\left[S(\tau+1) e^{\rho \Delta t}-S(\tau) e^{r \Delta t}\right],
$$

ou:

$$
V(\tau)=e^{r \Delta t} V(\tau-1)+u(\tau-1)\left[S(\tau) e^{\rho \Delta t}-S(\tau-1) e^{r \Delta t}\right],
$$

o que completa a prova.

Lema A.9. Seja $S^{P}(\cdot)$, preço do ativo de risco com relação à medida de probabilidade P, tal como definido na Equação (4.26). Então, $S^{Q_{l}}(\mathcal{T})$, o preço do 
ativo de risco com relação à medida de probabilidade $Q_{l}$ definida em (A.8), pode ser escrito como:

$$
\begin{aligned}
S^{Q_{l}}(\mathcal{T})=S(\tau) e^{\left[(\mu-\rho)(\mathcal{T}-\tau)+\sigma^{2} p\right] \Delta t} & \frac{\exp \left[\left(1-e^{\beta_{Y}+\frac{1}{2} \sigma_{Y}^{2}}\right) \lambda^{P}(\mathcal{T}-\tau) \Delta t\right]}{\exp \left[\left(1-e^{\beta_{Y}+\frac{3}{2} \sigma_{Y}^{2}}\right) \lambda^{Q}(\mathcal{T}-\tau) \Delta t\right]} \\
& Z_{3}((\mathcal{T}-\tau) \Delta t) Z_{4}((\mathcal{T}-\tau) \Delta t), \quad(\mathrm{A}
\end{aligned}
$$

onde:

$$
\begin{gathered}
Z_{3}((\mathcal{T}-\tau) \Delta t)=\exp \left[\sum_{j=1}^{\mathcal{T}-\tau} \sigma \Delta W^{Q_{l}}(\tau+j)-\frac{1}{2} \sigma^{2} \Delta t\right] \\
Z_{4}((\mathcal{T}-\tau) \Delta t)=e^{\left(\lambda^{Q}-\lambda^{R}\right)(\mathcal{T}-\tau) \Delta t} \prod_{i=1}^{N^{Q}((\mathcal{T}-\tau) \Delta t)} \frac{\lambda^{R} f^{R}\left(Y_{i}^{Q}\right)}{\lambda^{Q} f^{Q}\left(Y_{i}^{Q}\right)}, \\
\lambda^{Q}=\lambda^{P} e^{\frac{1}{2} \sigma_{Y}^{2}+\beta_{Y}} \\
\lambda^{R}=\lambda^{Q} e^{\beta_{Y}+\frac{3}{2} \sigma_{Y}^{2}} \\
f^{Q}\left(Y_{i}^{Q}\right)=\frac{1}{\sqrt{2 \pi} \sigma_{Y}} e^{-\frac{1}{2}\left(\frac{Y_{i}^{Q}-\beta_{Y}}{\sigma_{Y}}\right)^{2}} \\
f^{R}\left(Y_{i}^{Q}\right)=\frac{1}{\sqrt{2 \pi} \sigma_{Y}} e^{-\frac{1}{2}\left(\frac{Y_{i}^{Q}-\left(\sigma_{Y}^{2}+\beta_{Y}\right)}{\sigma_{Y}}\right)^{2}} .
\end{gathered}
$$

Demonstração. Escrevendo $S^{P}\left(t+\Delta t_{t}\right)$, definido na Equação (4.26), com a notação para tempo discreto, obtemos:

$$
\begin{aligned}
& S^{P}(\tau+1)= \\
& S(\tau) \exp \left\{\sigma \Delta W^{P}(\tau+1)+\left(\mu-\rho-\left(e^{\beta_{Y}+\frac{1}{2} \sigma_{Y}^{2}}-1\right) \lambda^{P}-\frac{1}{2} \sigma^{2}\right) \Delta t\right\} \prod_{i=1}^{N^{P}(\Delta t)} e^{Y_{i}^{P}} .
\end{aligned}
$$

Aplicando esta expressão sucessivamente, obtemos $S^{P}(\mathcal{T})$ em relação à medida de probabilidade $P$ :

$$
\begin{gathered}
S^{P}(\mathcal{T})= \\
S(\tau) \exp \left[\sum_{j=1}^{\mathcal{T}-\tau} \sigma \Delta W^{P}(\tau+j)+\sum_{j=1}^{\mathcal{T}-\tau}\left(\mu-\rho-\left(e^{\beta_{Y}+\frac{1}{2} \sigma_{Y}^{2}}-1\right) \lambda^{P}-\frac{1}{2} \sigma^{2}\right) \Delta t\right] \\
\prod_{i=1}^{N^{P}((\mathcal{T}-\tau) \Delta t)} e^{Y_{i}^{P}} . \quad(\mathrm{A} .236)
\end{gathered}
$$


Reagrupando:

$$
\begin{array}{r}
S^{P}(\mathcal{T})= \\
S(\tau) \exp \left[\sum_{j=1}^{\mathcal{T}-\tau}\left(\sigma \Delta W^{P}(\tau+j)-\frac{1}{2} \sigma^{2} \Delta t\right)\right] \exp \left\{\sum_{j=1}^{\mathcal{T}-\tau}(\mu-\rho) \Delta t\right\} \\
\quad \exp \left[-\sum_{j=1}^{\mathcal{T}-\tau}\left(e^{\beta_{Y}+\frac{1}{2} \sigma_{Y}^{2}}-1\right) \lambda^{P} \Delta t\right] \prod_{i=1}^{N^{P}((\mathcal{T}-\tau) \Delta t)} e^{Y_{i}^{P}} .
\end{array}
$$

Simplificando somatórios:

$$
\begin{array}{r}
S^{P}(\mathcal{T})=S(\tau) e^{(\mu-\rho)(\mathcal{T}-\tau) \Delta t} \exp \left[\sum_{j=1}^{\mathcal{T}-\tau}\left(\sigma \Delta W^{P}(\tau+j)-\frac{1}{2} \sigma^{2} \Delta t\right)\right] \\
\underbrace{\exp \left[\left(1-e^{\beta_{Y}+\frac{1}{2} \sigma_{Y}^{2}}\right) \lambda^{P}(\mathcal{T}-\tau) \Delta t\right] \prod_{i=1}^{N^{P}((\mathcal{T}-\tau) \Delta t)} e^{Y_{i}^{P}}}_{Z_{2}((\mathcal{T}-\tau) \Delta t)} .
\end{array}
$$

Como mostrado na demonstração do Lema $4.1, Z_{2}((\mathcal{T}-\tau) \Delta t)$ pode ser escrito na forma (A.99), levando $S^{P}(\mathcal{T})$ na Equação (A.238) a ser escrito como:

$$
\begin{array}{r}
S^{P}(\mathcal{T})=S(\tau) e^{(\mu-\rho)(\mathcal{T}-\tau) \Delta t} \underbrace{\exp \left[\sum_{j=1}^{[\mathcal{T}-\tau}\left(\sigma \Delta W^{P}(\tau+j)-\frac{1}{2} \sigma^{2} \Delta t\right)\right]}_{Z_{1}((\mathcal{T}-\tau) \Delta t)} \\
\underbrace{e^{\left(\lambda^{P}-\lambda^{Q}\right)(\mathcal{T}-\tau) \Delta t} \prod_{i=1}^{N^{P}((\mathcal{T}-\tau) \Delta t)} \frac{\lambda^{Q} f^{Q}\left(Y_{i}^{P}\right)}{\lambda^{P} f^{P}\left(Y_{i}^{P}\right)}}_{Z_{2}((\mathcal{T}-\tau) \Delta t)} .
\end{array}
$$

com $f^{P}\left(Y_{i}^{P}\right), \lambda^{Q}$ e $f^{Q}\left(Y_{i}^{P}\right)$ dados, respectivamente, pelas Equações (A.100), (A.102), e (A.103).

Usando a mesma notação já introduzida no Lema (A.8):

$$
\begin{aligned}
& Z_{1}((\mathcal{T}-\tau) \Delta t)=\exp \left(\sum_{j=1}^{\mathcal{T}-\tau}\left(\sigma \Delta W^{P}(\tau+j)-\frac{1}{2} \sigma^{2} \Delta t\right)\right) \\
& Z_{2}((\mathcal{T}-\tau) \Delta t)=e^{\left(\lambda^{P}-\lambda^{Q}\right)(\mathcal{T}-\tau) \Delta t} \prod_{i=1}^{N^{P}((\mathcal{T}-\tau) \Delta t)} \frac{\lambda^{Q} f^{Q}\left(Y_{i}^{P}\right)}{\lambda^{P} f^{P}\left(Y_{i}^{P}\right)}
\end{aligned}
$$

a Equação (A.239) pode ser escrita como:

$$
S^{P}(\mathcal{T})=S(\tau) e^{(\mu-\rho)(\mathcal{T}-\tau) \Delta t} Z_{1}((\mathcal{T}-\tau) \Delta t) Z_{2}((\mathcal{T}-\tau) \Delta t) .
$$


Em relação à medida de probabilidade $Q_{l}$ definida no Lema A.8, e usando os conceitos de mudança de medida apresentados na demonstração do Teorema $4.2, S^{P}(\mathcal{T})$ na Equação (A.238) pode ser escrito como:

$$
\begin{aligned}
S^{Q_{l}}(\mathcal{T})= & S(\tau) e^{(\mu-\rho)(\mathcal{T}-\tau) \Delta t} \\
\exp & {\left[\sum_{j=1}^{\mathcal{T}-\tau}\left(\sigma\left(\Delta W^{Q_{l}}(\tau+j)+\sigma_{l}(\tau+j-1) \Delta t\right)-\frac{1}{2} \sigma^{2} \Delta t\right)\right] } \\
& \exp \left[\left(1-e^{\beta_{Y}+\frac{1}{2} \sigma_{Y}^{2}}\right) \lambda^{P}(\mathcal{T}-\tau) \Delta t\right] \prod_{i=1}^{N^{Q}((\mathcal{T}-\tau) \Delta t)} e^{Y_{i}^{Q}+\sigma_{Y}^{2}} .
\end{aligned}
$$

Reagrupando alguns termos, a Equação (A.243) se torna:

$$
\begin{gathered}
S^{Q_{l}}(\mathcal{T})=S(\tau) e^{(\mu-\rho)(\mathcal{T}-\tau) \Delta t} \exp \left[\sum_{j=1}^{\mathcal{T}-\tau}\left(\sigma \sigma_{l}(\tau+j-1) \Delta t\right)\right] \\
\underbrace{\exp \left[\sum_{j=1}^{[\mathcal{T}-\tau} \sigma \Delta W^{Q_{l}}(\tau+j)-\frac{1}{2} \sigma^{2} \Delta t\right]}_{T_{1}} \\
\underbrace{\exp \left[\left(1-e^{\beta_{Y}+\frac{1}{2} \sigma_{Y}^{2}}\right) \lambda^{P}(\mathcal{T}-\tau) \Delta t\right]}_{Z_{3}((\mathcal{T}-\tau) \Delta t)} \underbrace{\prod_{T_{2}}^{N_{T}}}_{\prod_{i=1}^{N^{Q}((\mathcal{T}-\tau) \Delta t)} e^{Y_{i}^{Q}+\sigma_{Y}^{2}}} .
\end{gathered}
$$

O termo $T_{1} T_{2}$ acima não possui $E\left\{T_{1} T_{2}\right\}=1$, o que seria desejável. Usando a Equação (A.111), vemos que o termo $T_{2}$ possui valor esperado dado por:

$$
E^{P}\left\{\prod_{i=1}^{N^{Q}((\mathcal{T}-\tau) \Delta t)} e^{Y_{i}^{Q}+\sigma_{Y}^{2}}\right\}=\exp \left[\left(e^{\beta_{Y}+\frac{3}{2} \sigma_{Y}^{2}}-1\right) \lambda^{Q}(\mathcal{T}-\tau) \Delta t\right],
$$

de forma que:

$$
E^{P} \underbrace{\left\{\exp \left[\left(1-e^{\beta_{Y}+\frac{3}{2} \sigma_{Y}^{2}}\right) \lambda^{Q}(\mathcal{T}-\tau) \Delta t\right] \prod_{i=1}^{N^{Q}((\mathcal{T}-\tau) \Delta t)} e^{Y_{i}^{Q}+\sigma_{Y}^{2}}\right\}}_{Z_{4}((\mathcal{T}-\tau) \Delta t)}=1 . \quad \text { (A.246) }
$$

Multiplicando e dividindo a Equação (A.243) pela expressão no lado direito 
de (A.245), podemos rescrevê-la como:

$$
\begin{gathered}
S^{Q_{l}}(\mathcal{T})=S(\tau) e^{(\mu-\rho)(\mathcal{T}-\tau) \Delta t} \exp \left[\sum_{j=1}^{\mathcal{T}-\tau} \sigma \sigma_{l}(\tau+j-1) \Delta t\right] \\
\underbrace{\exp \left[\sum_{j=1}^{\mathcal{T}-\tau} \sigma \Delta W^{Q_{l}}(\tau+j)-\frac{1}{2} \sigma^{2} \Delta t\right]}_{Z_{3}((\mathcal{T}-\tau) \Delta t)} \frac{\exp \left[\left(1-e^{\beta_{Y}+\frac{1}{2} \sigma_{Y}^{2}}\right) \lambda^{P}(\mathcal{T}-\tau) \Delta t\right]}{\exp \left[\left(1-e^{\beta_{Y}+\frac{3}{2} \sigma_{Y}^{2}}\right) \lambda^{Q}(\mathcal{T}-\tau) \Delta t\right]} \\
\underbrace{\exp \left[\left(1-e^{\beta_{Y}+\frac{3}{2} \sigma_{Y}^{2}}\right) \lambda^{Q}(\mathcal{T}-\tau) \Delta t\right] \prod_{i=1}^{N^{Q}((\mathcal{T}-\tau) \Delta t)} e^{Y_{i}^{Q}+\sigma_{Y}^{2}}}_{Z_{4}((\mathcal{T}-\tau) \Delta t)}
\end{gathered}
$$

Usando as Equações (A.174) e (A.175), podemos escrever:

$$
\exp \left[\sum_{j=1}^{\mathcal{T}-\tau} \sigma \sigma_{l}(\tau+j-1) \Delta t\right]=\exp \left[\sum_{j=1}^{\mathcal{T}-\tau} \sigma \sigma \mathbf{I}_{C_{p, l}^{n}}(j) \Delta t\right]=e^{\sigma^{2} p \Delta t},
$$

e a Equação (A.247) fica:

$$
\begin{gathered}
S^{Q_{l}}(\mathcal{T})=S(\tau) e^{\left[(\mu-\rho)(\mathcal{T}-\tau)+\sigma^{2} p\right] \Delta t} \frac{\exp \left[\left(1-e^{\beta_{Y}+\frac{1}{2} \sigma_{Y}^{2}}\right) \lambda^{P}(\mathcal{T}-\tau) \Delta t\right]}{\exp \left[\left(1-e^{\beta_{Y}+\frac{3}{2} \sigma_{Y}^{2}}\right) \lambda^{Q}(\mathcal{T}-\tau) \Delta t\right]} \\
\underbrace{\exp \left\{\sum_{j=1}^{\{\mathcal{T}-\tau}\left(\sigma \Delta W^{Q_{l}}(\tau+j)-\frac{1}{2} \sigma^{2} \Delta t\right)\right\}}_{Z_{3}((\mathcal{T}-\tau) \Delta t)} \\
\underbrace{\exp \left[\left(1-e^{\beta_{Y}+\frac{3}{2} \sigma_{Y}^{2}}\right) \lambda^{Q}(\mathcal{T}-\tau) \Delta t\right] \prod_{i=1}^{N^{Q}((\mathcal{T}-\tau) \Delta t)} e^{Y_{i}^{Q}+\sigma_{Y}^{2}}}_{Z_{4}((\mathcal{T}-\tau) \Delta t)},
\end{gathered}
$$

ou, simplesmente:

$$
\begin{aligned}
S^{Q_{l}}(\mathcal{T})=S(\tau) e^{\left[(\mu-\rho)(\mathcal{T}-\tau)+\sigma^{2} p\right] \Delta t} & \frac{\exp \left[\left(1-e^{\beta_{Y}+\frac{1}{2} \sigma_{Y}^{2}}\right) \lambda^{P}(\mathcal{T}-\tau) \Delta t\right]}{\exp \left[\left(1-e^{\beta_{Y}+\frac{3}{2} \sigma_{Y}^{2}}\right) \lambda^{Q}(\mathcal{T}-\tau) \Delta t\right]} \\
& Z_{3}((\mathcal{T}-\tau) \Delta t) Z_{4}((\mathcal{T}-\tau) \Delta t) .
\end{aligned}
$$

Como passo final para completar a prova, escrevamos $Z_{4}((\mathcal{T}-\tau) \Delta t)$ numa forma semelhante à da Equação (A.240). Procedamos de forma análoga à feita 
na demonstração do Lema 4.1, ou seja, procuremos $\lambda^{R}$ e $f^{R}\left(Y_{i}\right)$ tais que:

$$
\begin{aligned}
e^{\left(\lambda^{Q}-\lambda^{R}\right)(\mathcal{T}-\tau) \Delta t} \prod_{i=1}^{N^{Q}((\mathcal{T}-\tau) \Delta t)} \frac{\lambda^{R} f^{R}\left(Y_{i}^{Q}\right)}{\lambda^{Q} f^{Q}\left(Y_{i}^{Q}\right)}= & \underbrace{\exp \left[\left(1-e^{\beta_{Y}+\frac{3}{2} \sigma_{Y}^{2}}\right) \lambda^{Q}(\mathcal{T}-\tau) \Delta t\right] \prod_{i=1}^{N^{Q}((\mathcal{T}-\tau) \Delta t)} e^{Y_{i}^{Q}+\sigma_{Y}^{2}}}_{Z_{4}((\mathcal{T}-\tau) \Delta t)} .
\end{aligned}
$$

Sabemos que:

$$
f^{Q}\left(Y_{i}^{Q}\right)=\frac{1}{\sqrt{2 \pi} \sigma_{Y}} e^{-\frac{1}{2}\left(\frac{Y_{i}^{Q}-\beta_{Y}}{\sigma_{Y}}\right)^{2}} .
$$

Portanto, a Equação (A.251) fica:

$$
\begin{gathered}
e^{\left(\lambda^{Q}-\lambda^{R}\right)(\mathcal{T}-\tau) \Delta t} \prod_{i=1}^{N^{Q}((\mathcal{T}-\tau) \Delta t)} \frac{\lambda^{R} f^{R}\left(Y_{i}^{Q}\right)}{\frac{\lambda^{Q}}{\sqrt{2 \pi} \sigma_{Y}} \exp \left[-\frac{1}{2}\left(\frac{Y_{i}^{Q}-\beta_{Y}}{\sigma_{Y}}\right)^{2}\right]}= \\
\exp \left[\left(1-e^{\beta_{Y}+\frac{3}{2} \sigma_{Y}^{2}}\right) \lambda^{Q}(\mathcal{T}-\tau) \Delta t\right] \prod_{i=1}^{N^{Q}((\mathcal{T}-\tau) \Delta t)} e^{Y_{i}^{Q}+\sigma_{Y}^{2}},
\end{gathered}
$$

que é satisfeita se:

$e^{\left(\lambda^{Q}-\lambda^{R}\right)(\mathcal{T}-\tau) \Delta t}=\exp \left[\left(1-e^{\beta_{Y}+\frac{3}{2} \sigma_{Y}^{2}}\right) \lambda^{Q}(\mathcal{T}-\tau) \Delta t\right] \Longrightarrow \lambda^{R}=\lambda^{Q} e^{\beta_{Y}+\frac{3}{2} \sigma_{Y}^{2}}$,

e se:

$$
\begin{gathered}
\frac{\lambda^{R} f^{R}\left(Y_{i}^{Q}\right)}{\frac{\lambda^{Q}}{\sqrt{2 \pi} \sigma_{Y}} \exp \left(-\frac{1}{2}\left(\frac{Y_{i}^{Q}-\beta_{Y}}{\sigma_{Y}}\right)^{2}\right)}=e^{\left(Y_{i}^{Q}+\sigma_{Y}^{2}\right)} \Longrightarrow \\
f^{R}\left(Y_{i}^{Q}\right)=\frac{1}{\sqrt{2 \pi} \sigma_{Y}} e^{-\frac{1}{2}\left(\frac{Y_{i}^{Q}-\left(\sigma_{Y}^{2}+\beta_{Y}\right)}{\sigma_{Y}}\right)^{2}} .
\end{gathered}
$$

Assim, realmente escrevemos $Z_{4}((\mathcal{T}-\tau) \Delta t)$ na forma definida pela Equação (A.251), com $f^{Q}\left(Y_{i}^{Q}\right), \lambda^{R}$ e $f^{R}\left(Y_{i}^{Q}\right)$ dados, respectivamente, pelas Equações (A.252), (A.254) e (A.255), o que completa a prova.

Demonstração do Lema 5.1. Conforme demonstração do Lema A.9:

$$
\begin{gathered}
S^{P}(\mathcal{T})= \\
S(\tau) \exp \left[\sum_{j=1}^{\mathcal{T}-\tau} \sigma \Delta W^{P}(\tau+j)+\sum_{j=1}^{\mathcal{T}-\tau}\left(\mu-\rho-\left(e^{\beta_{Y}+\frac{1}{2} \sigma_{Y}^{2}}-1\right) \lambda^{P}-\frac{1}{2} \sigma^{2}\right) \Delta t\right] \\
\underbrace{\prod_{i=1}^{N^{P}((\mathcal{T}-\tau) \Delta t)} e^{Y_{i}^{P}}}_{K_{1}} \cdot(\mathrm{A} .256)
\end{gathered}
$$


O termo $K_{1}$ pode ser escrito como:

$$
K_{1}=\prod_{i=1}^{N^{P}((\mathcal{T}-\tau) \Delta t)} e^{Y_{i}^{P}}=\exp \left[\sum_{i=1}^{N^{P}((\mathcal{T}-\tau) \Delta t)} Y_{i}^{P}\right]
$$

Usando a Equação (A.257), reagrupando termos e simplificando somatórios, a Expressão (A.256) pode ser rescrita como:

$$
\begin{aligned}
& S^{P}(\mathcal{T})=S(\tau) \exp \left[\sum_{j=1}^{\mathcal{T}-\tau} \sigma \Delta W^{P}(\tau+j)+\right. \\
& \left.\quad \sum_{i=1}^{N^{P}((\mathcal{T}-\tau) \Delta t)} Y_{i}^{P}+\left(\mu-\rho-\left(e^{\beta_{Y}+\frac{1}{2} \sigma_{Y}^{2}}-1\right) \lambda^{P}-\frac{1}{2} \sigma^{2}\right)(\mathcal{T}-\tau) \Delta t\right] .
\end{aligned}
$$

Em relação à medida de probabilidade $Q_{l}$ definida no Lema A.8, e usando os conceitos de mudança de medida apresentados na demonstração do Teorema $4.2, S^{P}(\mathcal{T})$ na Equação (A.258) pode ser escrito como:

$$
\begin{aligned}
S^{Q_{l}}(\mathcal{T})=S(\tau) \exp & {\left[\sum_{j=1}^{\mathcal{T}-\tau} \sigma\left(\Delta W^{Q_{l}}(\tau+j)+\sigma_{l}(\tau+j-1) \Delta t\right)+\right.} \\
& \sum_{i=1}^{N^{Q}((\mathcal{T}-\tau) \Delta t)}\left(Y_{i}^{Q}+\sigma_{Y}^{2}\right)+\prod_{i=1}^{N^{Q}((\mathcal{T}-\tau) \Delta t)} e^{Y_{i}^{Q}+\sigma_{Y}^{2}}+ \\
& \left.\left(\mu-\rho-\left(e^{\beta_{Y}+\frac{1}{2} \sigma_{Y}^{2}}-1\right) \lambda^{P}-\frac{1}{2} \sigma^{2}\right)(\mathcal{T}-\tau) \Delta t\right] .
\end{aligned}
$$

Usando as equações (A.174) e (A.175), podemos escrever:

$$
\sum_{j=1}^{\mathcal{T}-\tau} \sigma \sigma_{l}(\tau+j-1) \Delta t=\sum_{j=1}^{\mathcal{T}-\tau} \sigma \sigma \mathbf{I}_{C_{p, l}^{n}}(j) \Delta t=\sigma^{2} p \Delta t .
$$

Portanto, $S^{Q_{l}}(\mathcal{T})$ na Equação (A.259) pode ser rescrito como:

$$
\begin{aligned}
& S^{Q_{l}}(\mathcal{T})= \\
& S(\tau) \exp \left[\sum_{j=1}^{\mathcal{T}-\tau} \sigma \Delta W^{Q_{l}}(\tau+j)+\sum_{i=1}^{N^{Q}((\mathcal{T}-\tau) \Delta t)}\left(Y_{i}^{Q}+\sigma_{Y}^{2}\right)+\right. \\
& \left.\quad\left(\mu-\rho-\left(e^{\beta_{Y}+\frac{1}{2} \sigma_{Y}^{2}}-1\right) \lambda^{P}-\frac{1}{2} \sigma^{2}\right)(\mathcal{T}-\tau) \Delta t+\sigma^{2} p \Delta t\right],
\end{aligned}
$$

completando a prova.

Lema A.10. Seja $S^{Q_{l}}(\mathcal{T})$, preço do ativo de risco com relação à medida de probabilidade $Q_{l}$, tal como definido na Equação (5.2). Então, $S^{R}(\mathcal{T})$, o preço do ativo de risco com relação à medida de probabilidade $R$ dada por:

$$
m_{\mathcal{T}, \tau}^{Q_{l} \rightarrow R}=Z_{3}((\mathcal{T}-\tau) \Delta t) Z_{4}((\mathcal{T}-\tau) \Delta t)
$$


onde:

$$
\begin{gathered}
Z_{3}((\mathcal{T}-\tau) \Delta t)=\exp \left[\sum_{j=1}^{\mathcal{T}-\tau} \sigma \Delta W^{Q_{l}}(\tau+j)-\frac{1}{2} \sigma^{2} \Delta t\right] \\
Z_{4}((\mathcal{T}-\tau) \Delta t)=e^{\left(\lambda^{Q}-\lambda^{R}\right)(\mathcal{T}-\tau) \Delta t} \prod_{i=1}^{N^{Q}((\mathcal{T}-\tau) \Delta t)} \frac{\lambda^{R} f^{R}\left(Y_{i}^{Q}\right)}{\lambda^{Q} f^{Q}\left(Y_{i}^{Q}\right)}, \\
\lambda^{Q}=\lambda^{P} e^{\frac{1}{2} \sigma_{Y}^{2}+\beta_{Y}} \\
\lambda^{R}=\lambda^{Q} e^{\beta_{Y}+\frac{3}{2} \sigma_{Y}^{2}} \\
f^{Q}\left(Y_{i}^{Q}\right)=\frac{1}{\sqrt{2 \pi} \sigma_{Y}} e^{-\frac{1}{2}\left(\frac{Y_{i}^{Q}-\beta_{Y}}{\sigma_{Y}}\right)^{2}}, \\
f^{R}\left(Y_{i}^{Q}\right)=\frac{1}{\sqrt{2 \pi} \sigma_{Y}} e^{-\frac{1}{2}\left(\frac{Y_{i}^{Q}-\left(\sigma_{Y}^{2}+\beta_{Y}\right)}{\sigma_{Y}}\right)^{2}}
\end{gathered}
$$

pode ser escrito como:

$$
\begin{aligned}
S^{R}(\mathcal{T})= & S(\tau) \exp \left[\sum_{j=1}^{\mathcal{T}-\tau} \sigma \Delta W^{R}(\tau+j)+\sum_{i=1}^{N^{R}((\mathcal{T}-\tau) \Delta t)}\left(Y_{i}^{R}+2 \sigma_{Y}^{2}\right)+\right. \\
& \left.\left(\mu-\rho-\left(e^{\beta_{Y}+\frac{1}{2} \sigma_{Y}^{2}}-1\right) \lambda^{P}+\frac{1}{2} \sigma^{2}\right)(\mathcal{T}-\tau) \Delta t+\sigma^{2} p \Delta t\right] .
\end{aligned}
$$

Demonstração. Devido à mudança de medida de probabilidade da medida $Q_{l}$ para a medida $R$, quando do cálculo de $E_{\tau}^{R}\{\cdot\}$, as variáveis aleatórias $\Delta W^{Q_{l}}(\tau+$ $j$ ) são substituídas por $\Delta W^{R}(\tau+j)$, que permanecem independentes e identicamente distribuídas sob a nova medida $R$, respeitando-se as seguintes relações (dadas pelo teorema de Girsanov):

$$
\begin{gathered}
\Delta W^{Q_{l}}(\tau+j)=\Delta W^{R}(\tau+j)+\sigma \Delta t, \\
\Delta W^{Q_{l}}(\tau+j) N(0, \Delta t) \text { com respeito à medida } Q_{l}, \\
\Delta W^{Q_{l}}(\tau+j) N(\sigma \Delta t, \Delta t) \quad \text { com respeito à medida } R, \\
\Delta W^{R}(\tau+j) N(0, \Delta t) \text { com respeito à medida } R .
\end{gathered}
$$

As variáveis aleatórias $Y_{i}^{Q}$ são substituídas por $Y_{i}^{R}$, que permanecem independentes e identicamente distribuídas sob a nova medida $Q_{l}$, respeitando-se as seguintes relações:

$$
\begin{gathered}
Y_{i}^{Q}=Y_{i}^{R}+\sigma_{Y}^{2}, \\
Y_{i}^{Q} \sim N\left(\beta_{Y}, \sigma_{Y}^{2}\right) \text { com respeito à medida } Q_{l}, \\
Y_{i}^{Q} \sim N\left(\sigma_{Y}^{2}+\beta_{Y}, \sigma_{Y}^{2}\right) \text { com respeito à medida } R, \\
Y_{i}^{R} \sim N\left(\beta_{Y}, \sigma_{Y}^{2}\right) \text { com respeito à medida } R .
\end{gathered}
$$


Além disso, o processo de Poisson $N^{Q}(\cdot)$, de intensidade $\lambda^{Q}$ com relação à medida de probabilidade $Q_{l}$, é substituído por um novo processo de Poisson $N^{R}(\cdot)$, de intensidade $\lambda^{R}=\lambda^{Q} e^{\beta_{Y}+\frac{3}{2} \sigma_{Y}^{2}}$ com relação à medida de probabilidade $R$.

Portanto, usando esses conceitos, $S^{Q_{l}}(\mathcal{T})$ definido na Equação $(5.2)$ pode ser rescrito como:

$$
\begin{array}{r}
S^{R}(\mathcal{T})=S(\tau) \exp \left[\sum_{j=1}^{\mathcal{T}-\tau} \sigma\left(\Delta W^{R}(\tau+j)+\sigma \Delta t\right)+\sum_{i=1}^{N^{R}((\mathcal{T}-\tau) \Delta t)}\left(Y_{i}^{R}+2 \sigma_{Y}^{2}\right)+\right. \\
\left.\left(\mu-\rho-\left(e^{\beta_{Y}+\frac{1}{2} \sigma_{Y}^{2}}-1\right) \lambda^{P}-\frac{1}{2} \sigma^{2}\right)(\mathcal{T}-\tau) \Delta t+\sigma^{2} p \Delta t\right] . \quad(\text { A. } 270)
\end{array}
$$

Reagrupando alguns termos, a Equação (A.270) se transforma em:

$$
\begin{aligned}
S^{R}(\mathcal{T}) & = \\
& S(\tau) \exp \left[\sum_{j=1}^{\mathcal{T}-\tau} \sigma \Delta W^{R}(\tau+j)+\sum_{i=1}^{N^{R}((\mathcal{T}-\tau) \Delta t)}\left(Y_{i}^{R}+2 \sigma_{Y}^{2}\right)+\right. \\
& \left.\left(\mu-\rho-\left(e^{\beta_{Y}+\frac{1}{2} \sigma_{Y}^{2}}-1\right) \lambda^{P}+\frac{1}{2} \sigma^{2}\right)(\mathcal{T}-\tau) \Delta t+\sigma^{2} p \Delta t\right],
\end{aligned}
$$

completando a prova.

Demonstração do Teorema 5.2. Calculemos inicialmente $E_{\tau}^{Q_{l}}\{H(\mathcal{T})\}$. Usando a Equação (5.47) e as propriedades do operador linear esperança, obtemos:

$$
\begin{aligned}
E_{\tau}^{Q_{l}}\{H(\mathcal{T})\}= & E_{\tau}^{Q_{l}}\left\{\left(S^{Q_{l}}(\mathcal{T})-K\right) \mathbf{I}_{\left\{S^{Q_{l}}(\mathcal{T})>K\right\}}\right\}= \\
& E_{\tau}^{Q_{l}}\left\{S^{Q_{l}}(\mathcal{T}) \mathbf{I}_{\left\{S^{Q_{l}}(\mathcal{T})>K\right.}\right\}-K E_{\tau}^{Q_{l}}\left\{\mathbf{I}_{\left\{S^{Q_{l}}(\mathcal{T})>K\right\}}\right\}
\end{aligned}
$$

Cálculo de $E_{l, \tau}^{Q_{l}}\left\{\mathbf{I}_{\left\{S^{\left.Q_{l}(\mathcal{T})>K\right\}}\right\}}\right\}$

Usando a lei das expectativas iteradas:

$$
\begin{array}{r}
E_{l, \tau}^{Q_{l}}\left\{\mathbf{I}_{\left\{S^{Q_{l}(\mathcal{T})>K}\right\}}\right\}=E_{l, \tau}^{Q_{l}}\left\{E_{l, \tau}^{Q_{l}}\left\{\mathbf{I}_{\left\{S^{Q_{l}}(\mathcal{T})>K\right.} \mid N^{Q}((\mathcal{T}-\tau) \Delta t)=\eta\right\}\right\}= \\
\left.E_{l, \tau}^{Q_{l}}\left\{\operatorname{Prob} .\left\{S^{Q_{l}}(\mathcal{T})>K \mid N^{Q}((\mathcal{T}-\tau) \Delta t)=\eta\right\}\right\} \quad \text { A. } 273\right)
\end{array}
$$

Usando a Equação $(5.2)$, a condição $\left\{S^{Q_{l}}(\mathcal{T})>K \mid N^{Q}((\mathcal{T}-\tau) \Delta t)=\eta\right\}$ pode ser escrita como:

$$
\begin{aligned}
& S(\tau) \exp \left[\sum_{j=1}^{\mathcal{T}-\tau} \sigma \Delta W^{Q}(\tau+j)+\sum_{i=1}^{\eta}\left(Y_{i}^{Q}+\sigma_{Y}^{2}\right)+\right. \\
& \left.\quad\left(\mu-\rho-\left(e^{\beta_{Y}+\frac{1}{2} \sigma_{Y}^{2}}-1\right) \lambda^{P}-\frac{1}{2} \sigma^{2}\right)(\mathcal{T}-\tau) \Delta t+\sigma^{2} p \Delta t\right]>K .
\end{aligned}
$$


Esta expressão pode ser rescrita como:

$$
\begin{aligned}
& \underbrace{-\left(\sum_{j=1}^{\mathcal{T}-\tau} \sigma \Delta W^{Q}(\tau+j)+\sum_{i=1}^{\eta} Y_{i}^{Q}\right)}_{\xi_{1}}<\ln \left(\frac{S(\tau)}{K}\right)+ \\
& \left(\mu-\rho-\left(e^{\beta_{Y}+\frac{1}{2} \sigma_{Y}^{2}}-1\right) \lambda^{P}-\frac{1}{2} \sigma^{2}\right)(\mathcal{T}-\tau) \Delta t+\sigma^{2} p \Delta t+\eta \sigma_{Y}^{2} .
\end{aligned}
$$

A variável aleatória $\xi_{1}$ na expressão acima é normalmente distribuída, pois corresponde a uma soma de variáveis normalmente distribuídas. Além disso, a média e a variância de $\xi_{1}$ são dadas por:

$$
\begin{gathered}
E_{l, \tau}^{Q}\left\{\xi_{1}\right\}=E_{l, \tau}^{Q}\left\{-\left(\sum_{j=1}^{\mathcal{T}-\tau} \sigma \Delta W^{Q}(\tau+j)+\sum_{i=1}^{\eta} Y_{i}^{Q}\right)\right\}=-\eta \beta_{Y}, \\
\left(\sigma_{\tau}^{Q}\right)^{2}\left\{\xi_{1}\right\}=\left(\sigma_{\tau}^{Q}\right)^{2}\left\{-\left(\begin{array}{ll}
\left.\left.\sum_{j=1}^{\mathcal{T}-\tau} \sigma \Delta W^{Q}(\tau+j)+\sum_{i=1}^{\eta} Y_{i}^{Q}\right)\right\}= \\
\sigma^{2}(\mathcal{T}-\tau) \Delta t+\eta \sigma_{Y}^{2} .
\end{array}\right.\right.
\end{gathered}
$$

Subtraindo $E_{l, \tau}^{Q}\left\{\xi_{1}\right\}$ de ambos os lados da Igualdade (A.275), e depois dividindo ambos os lados da expressão resultante por $\sigma_{\tau}^{Q}\left\{\xi_{1}\right\}$, obtemos:

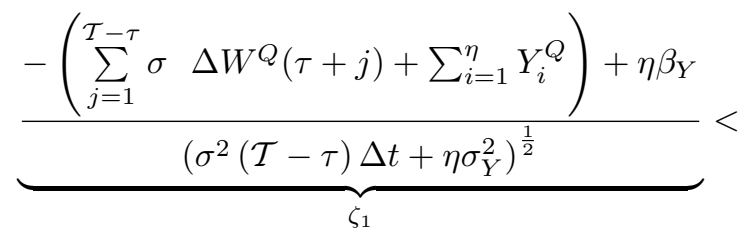

$$
\begin{aligned}
& \underbrace{\frac{\ln \left(\frac{S(\tau)}{K}\right)+\left(\mu-\rho-\left(e^{\beta_{Y}+\frac{1}{2} \sigma_{Y}^{2}}-1\right) \lambda^{P}-\frac{1}{2} \sigma^{2}\right)(\mathcal{T}-\tau) \Delta t+\sigma^{2} p \Delta t+\eta\left(\sigma_{Y}^{2}+\beta_{Y}\right)}{\left(\sigma^{2}(\mathcal{T}-\tau) \Delta t+\eta \sigma_{Y}^{2}\right)^{\frac{1}{2}}}}_{d_{Q, p, \eta}} .
\end{aligned}
$$

A variável aleatória $\zeta_{1}$ definida na Equação (A.278) acima, dada por:

$$
\zeta_{1}=\frac{\xi_{1}-E_{l, \tau}^{Q}\left\{\xi_{1}\right\}}{\sigma_{\tau}^{Q}\left\{\xi_{1}\right\}}=\frac{-\left(\sum_{j=1}^{\mathcal{T}-\tau} \sigma \Delta W^{Q}(\tau+j)+\sum_{i=1}^{\eta} Y_{i}^{Q}\right)+\eta \beta_{Y}}{\left(\sigma^{2}(\mathcal{T}-\tau) \Delta t+\eta \sigma_{Y}^{2}\right)^{\frac{1}{2}}},
$$

é uma variável aleatória com distribuição normal padrão, i.e., com distribuição normal de média nula e variância unitária. Portanto, a condição $\left\{S^{Q_{l}}(\mathcal{T})>\right.$ $\left.K \mid N^{Q}((\mathcal{T}-\tau) \Delta t)=\eta\right\}$ se traduz simplesmente em:

$$
\zeta_{1}<d_{Q, p, \eta}
$$


e Prob. $\left\{S^{Q_{l}}(\mathcal{T})>K \mid N^{Q}((\mathcal{T}-\tau) \Delta t)=\eta\right\}$ pode ser calculado simplesmente como:

$\operatorname{Prob} \cdot\left\{S^{Q_{l}}(\mathcal{T})>K \mid N^{Q}((\mathcal{T}-\tau) \Delta t)=\eta\right\}=$

$$
\operatorname{Prob}\left\{\zeta_{1}<d_{Q, p, \eta}\right\}=\frac{1}{\sqrt{2 \pi}} \int_{-\infty}^{d_{Q, p, \eta}} e^{-\frac{x^{2}}{2}} d x=\phi\left(d_{Q, p, \eta}\right) .
$$

Assim, voltando à Expressão (A.273), temos:

$$
\begin{aligned}
& E_{l, \tau}^{Q_{l}}\left\{\mathbf{I}_{\left\{S^{Q_{l}}(\mathcal{T})>K\right\}}\right\}= \\
& E_{l, \tau}^{Q_{l}}\left\{E_{l, \tau}^{Q_{l}}\left\{\mathbf{I}_{\left\{S^{Q_{l}}(\mathcal{T})>K\right\}} \mid N^{Q}((\mathcal{T}-\tau) \Delta t)=\eta\right\}\right\}= \\
& E_{l, \tau}^{Q_{l}}\left\{\operatorname{Prob} .\left\{S^{Q_{l}}(\mathcal{T})>K \mid N^{Q^{2}}((\mathcal{T}-\tau) \Delta t)=\eta\right\}\right\}=E_{l, \tau}^{Q_{l}}\left\{\phi\left(d_{Q, p, \eta}\right)\right\},
\end{aligned}
$$

e, finalmente:

$$
\begin{gathered}
E_{l, \tau}^{Q_{l}}\left\{\phi\left(d_{Q, p, \eta}\right)\right\}=\sum_{\eta=0}^{\infty}\left(\phi\left(d_{Q, p, \eta}\right) \operatorname{Prob} \cdot\left\{N^{Q}((\mathcal{T}-\tau) \Delta t)=\eta\right\}\right)= \\
\sum_{\eta=0}^{\infty}\left(\phi\left(d_{Q, p, \eta}\right) \frac{e^{-\lambda^{Q}(\mathcal{T}-\tau) \Delta t}\left(\lambda^{Q}(\mathcal{T}-\tau) \Delta t\right)^{\eta}}{\eta !}\right)= \\
\sum_{\eta=0}^{\infty}\left(\phi\left(d_{Q, p, \eta}\right) \frac{\exp \left[-\lambda^{P} e^{\frac{1}{2} \sigma_{Y}^{2}+\beta_{Y}}(\mathcal{T}-\tau) \Delta t\right]\left(\lambda^{P} e^{\frac{1}{2} \sigma_{Y}^{2}+\beta_{Y}}(\mathcal{T}-\tau) \Delta t\right)^{\eta}}{\eta !}\right)
\end{gathered}
$$

Cálculo de $E_{l, \tau}^{Q_{l}}\left\{S^{Q_{l}}(\mathcal{T}) \mathbf{I}_{\left\{S^{Q_{l}}(\mathcal{T})>K\right\}}\right\}$

Usando a Equação (A.228), $E_{l, \tau}^{Q_{l}}\left\{S^{Q_{l}}(\mathcal{T}) \mathbf{I}_{\left\{S^{Q_{l}}(\mathcal{T})>K\right\}}\right\}$ pode ser escrito como:

$$
\begin{aligned}
& E_{l, \tau}^{Q_{l}}\left\{S^{Q_{l}}(\mathcal{T}) \mathbf{I}_{\left\{S^{\left.Q_{l}(\mathcal{T})>K\right\}}\right\}}\right\}= \\
& E_{l, \tau}^{Q_{l}} \underbrace{\left\{S(\tau) e^{\left[(\mu-\rho)(\mathcal{T}-\tau)+\sigma^{2} p\right] \Delta t} \frac{\exp \left[\left(1-e^{\beta_{Y}+\frac{1}{2} \sigma_{Y}^{2}}\right) \lambda^{P}(\mathcal{T}-\tau) \Delta t\right]}{\exp \left[\left(1-e^{\beta_{Y}+\frac{3}{2} \sigma_{Y}^{2}}\right) \lambda^{Q}(\mathcal{T}-\tau) \Delta t\right]}\right.}_{T_{1}} \\
& \left.Z_{3}((\mathcal{T}-\tau) \Delta t) Z_{4}((\mathcal{T}-\tau) \Delta t) \mathbf{I}_{\left\{S^{Q_{l}}(\mathcal{T})>K\right\}}\right\}
\end{aligned}
$$

Colocando os termos conhecidos para fora da esperança, obtemos:

$$
\begin{aligned}
& E_{l, \tau}^{Q_{l}}\left\{S^{Q_{l}}(\mathcal{T}) \mathbf{I}_{\left\{S^{Q_{l}}(\mathcal{T})>K\right\}}\right\}= \\
& T_{1} E_{l, \tau}^{Q_{l}}\{\underbrace{Z_{3}((\mathcal{T}-\tau) \Delta t) Z_{4}((\mathcal{T}-\tau) \Delta t)}_{m_{\mathcal{T}, \tau}^{Q_{l} \rightarrow R}} \mathbf{I}_{\left\{S^{\left.Q_{l}(\mathcal{T})>K\right\}}\right\}}\}
\end{aligned}
$$


Conforme mostrado no livro Stochastic Calculus for Finance II [32], $m_{\mathcal{T}, \tau}^{Q_{l} \rightarrow R}$, tal como definido em (A.285), é a mudança de medida de probabilidade da medida $Q_{l}$ para a medida $R$, o que nos permite escrever a Equação (A.285) como:

$$
E_{l, \tau}^{Q_{l}}\left\{S^{Q_{l}}(\mathcal{T}) \mathbf{I}_{\left\{S^{Q_{l}}(\mathcal{T})>K\right\}}\right\}=T_{1} E_{l, \tau}^{R}\left\{\mathbf{I}_{\left\{S^{R}(\mathcal{T})>K\right\}}\right\}
$$

Usando a lei das expectativas iteradas:

$$
\begin{array}{r}
E_{l, \tau}^{R}\left\{\mathbf{I}_{\left\{S^{R}(\mathcal{T})>K\right\}}\right\}=E_{l, \tau}^{R}\left\{E_{l, \tau}^{R}\left\{\mathbf{I}_{\left\{S^{R}(\mathcal{T})>K\right\}} \mid N^{R}((\mathcal{T}-\tau) \Delta t)=\eta\right\}\right\}= \\
E_{l, \tau}^{R}\left\{\operatorname{Prob} \cdot\left\{S^{R}(\mathcal{T})>K \mid N^{R}((\mathcal{T}-\tau) \Delta t)=\eta\right\}\right\}
\end{array}
$$

Usando a Equação (A.269), a condição $\left\{S^{R}(\mathcal{T})>K \mid N^{R}((\mathcal{T}-\tau) \Delta t)=\eta\right\}$ pode ser escrita como:

$$
\begin{aligned}
& S(\tau) \exp \left[\sum_{j=1}^{\mathcal{T}-\tau} \sigma \Delta W^{R}(\tau+j)+\sum_{i=1}^{\eta}\left(Y_{i}^{R}+2 \sigma_{Y}^{2}\right)+\right. \\
& \left.\left(\mu-\rho-\left(e^{\beta_{Y}+\frac{1}{2} \sigma_{Y}^{2}}-1\right) \lambda^{P}+\frac{1}{2} \sigma^{2}\right)(\mathcal{T}-\tau) \Delta t+\sigma^{2} p \Delta t\right]>K .
\end{aligned}
$$

Esta expressão pode ser rescrita como:

$$
\begin{aligned}
& \underbrace{-\left(\sum_{j=1}^{\mathcal{T}-\tau} \sigma \Delta W^{R}(\tau+j)+\sum_{i=1}^{\eta} Y_{i}^{R}\right)}_{\xi_{2}}<\ln \left(\frac{S(\tau)}{K}\right)+ \\
& \left(\mu-\rho-\left(e^{\beta_{Y}+\frac{1}{2} \sigma_{Y}^{2}}-1\right) \lambda^{P}+\frac{1}{2} \sigma^{2}\right) \\
& (\mathcal{T}-\tau) \Delta t+\sigma^{2} p \Delta t+2 \eta \sigma_{Y}^{2}
\end{aligned}
$$

A variável aleatória $\xi_{2}$ na expressão acima é normalmente distribuída, pois corresponde a uma soma de variáveis normalmente distribuídas. Além disso, a média e a variância de $\xi_{2}$ são dadas por:

$$
\begin{array}{r}
E_{l, \tau}^{R}\left\{\xi_{2}\right\}=E_{l, \tau}^{R}\left\{-\left(\sum_{j=1}^{\mathcal{T}-\tau} \sigma \Delta W^{R}(\tau+j)+\sum_{i=1}^{\eta} Y_{i}^{R}\right)\right\}=-\eta \beta_{Y} \\
\left(\sigma_{\tau}^{R}\right)^{2}\left\{\xi_{2}\right\}=\left(\sigma_{\tau}^{R}\right)^{2}\left\{-\left(\sum_{j=1}^{\mathcal{T}-\tau} \sigma \Delta W^{R}(\tau+j)+\sum_{i=1}^{\eta} Y_{i}^{R}\right)\right\}= \\
\sigma^{2}(\mathcal{T}-\tau) \Delta t+\eta \sigma_{Y}^{2} .
\end{array}
$$

Subtraindo $E_{l, \tau}^{R}\left\{\xi_{2}\right\}$ de ambos os lados da Igualdade (A.289), e depois divi- 
dindo ambos os lados da expressão resultante por $\sigma_{\tau}^{R}\left\{\xi_{2}\right\}$, obtemos:

$$
\begin{aligned}
& \underbrace{\frac{-\left(\sum_{j=1}^{\mathcal{T}-\tau} \sigma \Delta W^{R}(\tau+j)+\sum_{i=1}^{\eta} Y_{i}^{R}\right)+\eta \beta_{Y}}{\left(\sigma^{2}(\mathcal{T}-\tau) \Delta t+\eta \sigma_{Y}^{2}\right)^{\frac{1}{2}}}<}_{\zeta_{2}} \\
& \underbrace{\frac{\ln \left(\frac{S(\tau)}{K}\right)+\left(\mu-\rho-\left(e^{\beta_{Y}+\frac{1}{2} \sigma_{Y}^{2}}-1\right) \lambda^{P}+\frac{1}{2} \sigma^{2}\right)(\mathcal{T}-\tau) \Delta t+\sigma^{2} p \Delta t+\eta\left(2 \sigma_{Y}^{2}+\beta_{Y}\right)}{\left(\sigma^{2}(\mathcal{T}-\tau) \Delta t+\eta \sigma_{Y}^{2}\right)^{\frac{1}{2}}}}_{d_{R, p, \eta}} .
\end{aligned}
$$

A variável aleatória $\zeta_{2}$ definida na Equação (A.292) acima, dada por:

$$
\zeta_{2}=\frac{\xi_{2}-E_{l, \tau}^{R}\left\{\xi_{2}\right\}}{\sigma_{\tau}^{R}\left\{\xi_{2}\right\}}=\frac{-\left(\sum_{j=1}^{\mathcal{T}-\tau} \sigma \Delta W^{R}(\tau+j)+\sum_{i=1}^{\eta} Y_{i}^{R}\right)+\eta \beta_{Y}}{\left(\sigma^{2}(\mathcal{T}-\tau) \Delta t+\eta \sigma_{Y}^{2}\right)^{\frac{1}{2}}}
$$

é uma variável aleatória com distribuição normal padrão, i.e., com distribuição normal de média nula e variância unitária. Portanto, a condição $\left\{S^{R}(\mathcal{T})>\right.$ $\left.K \mid N^{R}((\mathcal{T}-\tau) \Delta t)=\eta\right\}$ se traduz simplesmente em:

$$
\zeta_{2}<d_{R, p, \eta}
$$

e Prob. $\left\{S^{R}(\mathcal{T})>K \mid N^{R}((\mathcal{T}-\tau) \Delta t)=\eta\right\}$ pode ser calculado simplesmente como:

$\operatorname{Prob} .\left\{S^{R}(\mathcal{T})>K \mid N^{R}((\mathcal{T}-\tau) \Delta t)=\eta\right\}=\operatorname{Prob}\left\{\zeta_{2}<d_{R, p, \eta}\right\}=$

$$
\frac{1}{\sqrt{2 \pi}} \int_{-\infty}^{d_{R, p, \eta}} e^{-\frac{x^{2}}{2}} d x=\phi\left(d_{R, p, \eta}\right)
$$

Assim, voltando à Expressão (A.287), temos:

$$
\begin{gathered}
E_{l, \tau}^{R}\left\{\mathbf{I}_{\left\{S^{R}(\mathcal{T})>K\right\}}\right\}= \\
E_{l, \tau}^{R}\left\{E_{l, \tau}^{R}\left\{\mathbf{I}_{\left\{S^{R}(\mathcal{T})>K\right\}} \mid N^{R}((\mathcal{T}-\tau) \Delta t)=\eta\right\}\right\}= \\
E_{l, \tau}^{R}\left\{\operatorname{Prob} \cdot\left\{S^{R}(\mathcal{T})>K \mid N^{R}((\mathcal{T}-\tau) \Delta t)=\eta\right\}\right\}=E_{l, \tau}^{R}\left\{\phi\left(d_{R, p, \eta}\right)\right\},
\end{gathered}
$$


e:

$$
\begin{gathered}
E_{l, \tau}^{Q_{l}}\left\{\phi\left(d_{Q, p, \eta}\right)\right\}= \\
\sum_{\eta=0}^{\infty}\left(\phi\left(d_{R, p, \eta}\right) \operatorname{Prob} \cdot\left\{N^{R}((\mathcal{T}-\tau) \Delta t)=\eta\right\}\right)= \\
\sum_{\eta=0}^{\infty}\left(\phi\left(d_{R, p, \eta}\right) \frac{e^{-\lambda^{R}(\mathcal{T}-\tau) \Delta t}\left(\lambda^{R}(\mathcal{T}-\tau) \Delta t\right)^{\eta}}{\eta !}\right)= \\
\sum_{\eta=0}^{\infty}\left(\phi\left(d_{R, p, \eta}\right) \frac{\exp \left[-\lambda^{Q} e^{\beta_{Y}+\frac{3}{2} \sigma_{Y}^{2}}(\mathcal{T}-\tau) \Delta t\right]\left(\lambda^{Q} e^{\left.\beta_{Y}+\frac{3}{2} \sigma_{Y}^{2}(\mathcal{T}-\tau) \Delta t\right)^{\eta}}\right.}{\eta !}\right)= \\
\sum_{\eta=0}^{\infty}\left(\phi\left(d_{R, p, \eta}\right) \frac{\exp \left[-\lambda^{P}(\mathcal{T}-\tau) \Delta t e^{2\left(\sigma_{Y}^{2}+\beta_{Y}\right)}\right]\left(\lambda^{P}(\mathcal{T}-\tau) \Delta t e^{2\left(\sigma_{Y}^{2}+\beta_{Y}\right)}\right)^{\eta}}{\eta !}\right) .
\end{gathered}
$$

Cálculo de $E_{l, \tau}^{Q}\{H(\mathcal{T})\}$ :

Portanto, voltando à Equação (A.272) e juntando todos os termos obtidos anteriormente para o cálculo de $E_{l, \tau}^{Q}\{H(\mathcal{T})\}$, temos:

$$
E_{\tau}^{Q_{l}}\{H(\mathcal{T})\}=T_{1} G_{1}-K G_{2},
$$

onde:

$$
\begin{aligned}
& T_{1}=S(\tau) e^{\left[(\mu-\rho)(\mathcal{T}-\tau)+\sigma^{2} p\right] \Delta t} \frac{\exp \left[\left(1-e^{\beta_{Y}+\frac{1}{2} \sigma_{Y}^{2}}\right) \lambda^{P}(\mathcal{T}-\tau) \Delta t\right]}{\exp \left[\left(1-e^{\beta_{Y}+\frac{3}{2} \sigma_{Y}^{2}}\right) \lambda^{Q}(\mathcal{T}-\tau) \Delta t\right]}, \quad(\text { A.299 }) \\
& G_{1}= \\
& \sum_{\eta=0}^{\infty}\left(\phi\left(d_{R, p, \eta}\right) \frac{\exp \left[-\lambda^{P}(\mathcal{T}-\tau) \Delta t e^{2\left(\sigma_{Y}^{2}+\beta_{Y}\right)}\right]\left(\lambda^{P}(\mathcal{T}-\tau) \Delta t e^{2\left(\sigma_{Y}^{2}+\beta_{Y}\right)}\right)^{\eta}}{\eta !}\right),
\end{aligned}
$$

$$
\begin{aligned}
& G_{2}= \\
& \sum_{\eta=0}^{\infty}\left(\phi\left(d_{Q, p, \eta}\right) \frac{\exp \left[-\lambda^{P} e^{\frac{1}{2} \sigma_{Y}^{2}+\beta_{Y}}(\mathcal{T}-\tau) \Delta t\right]\left(\lambda^{P} e^{\frac{1}{2} \sigma_{Y}^{2}+\beta_{Y}}(\mathcal{T}-\tau) \Delta t\right)^{\eta}}{\eta !}\right) .
\end{aligned}
$$

Substituindo a Equação (A.298) na Equação (4.39), obtemos $H(\tau)$ :

$$
H(\tau)=e^{-r n \Delta t} \sum_{p=0}^{n}\left(a_{0}^{n-p} a_{1}^{p} \sum_{l=1}^{\left(\begin{array}{c}
n \\
p
\end{array}\right)} E_{\tau}^{Q_{l}}\{H(\mathcal{T})\}\right) .
$$


Notemos que $E_{\tau}^{Q_{l}}\{H(\mathcal{T})\}$, tal como definido pela Equação (A.298), não possui dependência em relação ao índice $l$ e, portanto, pode ser tirado do somatório. Assim, a Equação (A.302) se torna:

$$
H(\tau)=e^{-r n \Delta t} \sum_{p=0}^{n}\left[\left(\begin{array}{l}
n \\
p
\end{array}\right) a_{0}^{n-p} a_{1}^{p} E_{\tau}^{Q_{l}}\{H(\mathcal{T})\}\right],
$$

conforme enunciado no teorema, completando assim a prova.

Demonstração do Teorema 5.3. Para o cálculo de $\bar{u}(\tau)$ para a opção vanilla de compra, inicialmente devemos substituir as Equações (A.298) a (A.299) na Equação (4.44). Notando que $E_{\tau}^{Q_{l}}\{H(\mathcal{T})\}$ não possui dependência em relação ao índice $l$ e, portanto, pode ser tirado do somatório, a Equação (4.44) se torna:

$$
\begin{gathered}
\bar{u}(\tau)=\left(e^{(\mu-r) \Delta t}-1\right) \frac{A-V(\tau)}{B}, \\
A=\sum_{p=0}^{n}\left(e^{-r n \Delta t} a_{1}^{p} a_{0}^{n-p} E_{\tau}^{Q_{l}}\{H(\mathcal{T})\} \sum_{l=1}^{\left(\begin{array}{l}
n \\
p
\end{array}\right)}\left[\left(\frac{\alpha_{1}}{a_{1}}\right)^{\mathbf{I}_{C_{p, l}^{n}(1)}}\left(\frac{\alpha_{0}}{a_{0}}\right)^{\mathbf{I}_{C_{n-p, s}^{n}}^{(1)}}\right]\right), \\
B=S(\tau)\left\{\operatorname { e x p } \left[\left(2 \mu-2 r+\sigma^{2}+\right.\right.\right. \\
\left.\left.\left.\lambda^{P}\left(1-2 e^{\beta_{Y}+\frac{1}{2} \sigma_{Y}^{2}}+e^{2\left(\sigma_{Y}^{2}+\beta_{Y}\right)}\right)\right) \Delta t\right]-2 \exp [(\mu-r) \Delta t]+1\right\} .
\end{gathered}
$$

O somatório em $l$ na Expressão (A.304) pode ser expresso como:

$$
\sum_{l=1}^{\left(\begin{array}{c}
n \\
p
\end{array}\right)}\left[\left(\frac{\alpha_{1}}{a_{1}}\right)^{\mathbf{I}_{C_{p, l}^{n}(1)}^{(1)}}\left(\frac{\alpha_{0}}{a_{0}}\right)^{\mathbf{I}_{C_{n-p, s}^{n}}(1)}\right]=\left(\begin{array}{c}
n-1 \\
p
\end{array}\right)\left(\frac{\alpha_{0}}{a_{0}}\right)+\left(\begin{array}{c}
n-1 \\
n-p
\end{array}\right)\left(\frac{\alpha_{1}}{a_{1}}\right)
$$

onde:

$$
\left(\begin{array}{l}
s \\
k
\end{array}\right)=\left\{\begin{array}{ccc}
\frac{s !}{k !(s-k) !} & \text { se } & k \in\{0, \ldots, s\} \\
0 & \text { se } & k \notin\{0, \ldots, s\}
\end{array},\right.
$$

levando a Expressão (A.303) a:

$$
\begin{gathered}
\bar{u}(\tau)=\left(e^{(\mu-r) \Delta t}-1\right) \frac{A-V(\tau)}{S(\tau) B} \\
A=\sum_{p=0}^{n} e^{-r n \Delta t} a_{1}^{p} a_{0}^{n-p}\left[\left(\begin{array}{c}
n-1 \\
p
\end{array}\right)\left(\frac{\alpha_{0}}{a_{0}}\right)+\left(\begin{array}{c}
n-1 \\
n-p
\end{array}\right)\left(\frac{\alpha_{1}}{a_{1}}\right)\right] E_{\tau}^{Q_{l}}\{H(\mathcal{T})\} \\
B=S(\tau)\left\{\operatorname { e x p } \left[\left(2 \mu-2 r+\sigma^{2}+\right.\right.\right. \\
\left.\left.\left.\lambda^{P}\left(1-2 e^{\beta_{Y}+\frac{1}{2} \sigma_{Y}^{2}}+e^{2\left(\sigma_{Y}^{2}+\beta_{Y}\right)}\right)\right) \Delta t\right]-2 \exp [(\mu-r) \Delta t]+1\right\} .
\end{gathered}
$$

o que completa a prova. 
Demonstração do Teorema 5.4. A demonstração deste teorema é simples. Basta refazer a dedução do Teorema 5.2 substituindo $\lambda^{P}=0$ e $\eta=0$.

Demonstração do Teorema 5.5. A demonstração deste teorema é simples. Basta refazer a dedução do Teorema 5.3 substituindo $\lambda^{P}=0$ e $\eta=0$. 


\section{Referências Bibliográficas}

[1] T. Arai, An extension of mean-variance hedging to the discontinuous case, Finance and Stochastics 9 (2005), 129-139.

[2] D. Bertsimas, L. Kogan, and A. W. Lo, Hedging derivative securities and incomplete market: An epsilon-arbitrage approach, Operations Research 49 (2001), no. 3, 372-397.

[3] F. Black and M. Scholes, The pricing of options and corporate liabilities, Journal of Political Economy 81 (May-June 1973), 637-659.

[4] N. Bouleau and D. Lamberton, Residual risks and hedging strategies in markovian markets, Stochastic Processes and their Applications 33 (1989), $131-150$.

[5] A. Cerny, Dynamic programming and mean-variance hedging in discrete time, Applied Mathematical Finance 11 (2004), no. 1, 1-25.

[6] _ Mathematical techniques in finance: Tools for incomplete markets, Priceton University Press, 2004.

[7] R. Cont and P. Tankov, Financial modelling with jump processes, Chapman \& Hall, 2003.

[8] A. Dudenhausen, E. Schlögl, and L. Schlögl, Robustness of gaussian hedges and the hedging of fixed income derivatives, April, 1999.

[9] D. Duffie and H. Richardson, Mean-variance hedging in continuous time, Annals of Applied Probability 1 (1991), 1-15.

[10] P Embrechts, C. Klüppelberg, and T. Mikosch, Modelling extremal events for insurance and finance, Stochastic Modelling and Applied Probability, Springer, 2004.

[11] H. Fölmer and D. Sondermann, Hedging of non-redundant contingent claims, Contributions to Mathematical Economics (W. Hildenbrand and A. Mas-Colell, eds.), North-Holland, 1986, pp. 205-223.

[12] P. Glasserman, Monte carlo methods in financial engineering, Springer, 2003.

[13] C. Gourieroux, J. P. Laurent, and H. Pahm, Mean-variance hedging and numéraire, Mathematical Finance 8 (1998), no. 3, 179-200. 
[14] J. M. Harrison and S. R. Pliska, Martingales and stochastic integrals in the theory of continuous trading, Stochastic Processes and their applications 11 (1981), 215-260.

[15] H. Hsu, Schaum's outline of probability, random variables, and random processes, McGraw-Hill, 1996.

[16] J. C. Hull, Options, futures and other derivatives, 6th ed., Prentice Hall, 2005 .

[17] P. Jaeckel, Monte carlo methods in finance, John Wiley \& Sons, 2002.

[18] J. P. Laurent and H. Pham, Dynamic programming and mean-variance hedging, Finance and Stochastics 3 (1999), no. 1, 83-110.

[19] A. E. B. Lim, Quadratic hedging and mean-variance portfolio selection with random parameters in an incomplete market, Berkeley, University of California, 2003.

[20] R.C. Merton, Option pricing when underlying stock returns are discontinuous, Journal of Financial Economics 3 (January-March 1976), 125-144.

[21] M. Musiela and M. Rutkowsk, Martingale methods in financial modelling, Springer, 1998.

[22] A. Papoulis and S. U. Pillai, Probability, random variables and stochastic processes, 4th ed., McGraw-Hill Science/Engineering/Math, 2001.

[23] S. R. Pliska, Introduction to mathematical finance, Blackwell Publishers, 1997.

[24] T. Rheinländer and M. Schweizer, On $l^{2}$ projections on a space of stochastic integrals, Annals of Probability 25 (1997), no. 4, 1810-1831.

[25] L. C. G. Rogers and D. Williams, Diffusions, markov processes and martingales, vol. 1, Cambridge University Press, 2000.

[26] _ Diffusions, markov processes and martingales, vol. 2, Cambridge University Press, 2000.

[27] M. Schäl, On quadratic cost criteria for option hedging, Mathematics of Operations Research 19 (1994), no. 1, 121-131.

[28] M. Schweizer, Option hedging for semimartingales, Stochastic Processes and their Applications 37 (1991), 339-363.

[29] _ Variance-optimal hedging in discrete time, Mathematics of Operations Research 20 (1995), no. 1, 1-32.

[30] _ Approximation pricing and the variance-optimal martingale measure, The Annals of Applied Probability 24 (1996), no. 1, 206-236.

[31] _ Risky options simplified, International Journal of Theoretical and Applied Finance 2 (1999), no. 1, 59-82.

[32] S. E. Shreve, Stochastic calculus for finance ii, Springer, 2004. 
[33] N. N. Taleb, Dynamic hedging: Managing vanilla and exotic options, Wiley, 1997.

[34] D. Williams, Probability with martingales, Cambridge University Press, 1991.

[35] P. Wilmott, Paul wilmott on quantitative finance, 2nd ed., John Wiley \& Sons, 2006.

[36] X.Y. Zhou and D. Li, Continuous-time mean-variance portfolio selection: A stochastic lq framework, Applied Mathematical Optimization 42 (2000), 19-33. 